\title{
Influence of Prandtl Number and Effects of Disruption Shape on the Performance of Enhanced Tubes wit'? the Separation and Reattachment Mechanism
}

Energy Systems Division

Argonne National Laboratory



Operated by The University of Chicago, under Contract W-31-109-Eng-38, for the

United States Department of Energy 


\section{Argonne National Laboratory}

Argonne National Laboratory, with facilities in tire states of Illinois and Idaho, is owned by the United States govemment, and sperated by the University

of Chicago under the provisions of a contract with the Department of Energy.

This technical mport is a product of Argonne's Energy Systems Division.

For information on the division's scientific and engineering activities, contact:

Director, Energy Systems Division

Argonne National Laboratory

Argonne, Illinois 60439-4815

Telephone (708) 252-3724

\section{Disclaimer}

This report was prepared as an account of work sponsored by an agency of the United States Government. Neither the United States Govemment nor any agency thereof, nor any of their employees, makes any warranty, express or implied, or assumes any legal liability or responsibility for the accuracy, completeness, or usefulness of any information, apparatus, product, or process disclosed, or represents that its use would not infringe privately owned rights. Reierence herein to any specific commercial product, process, or service by trade name, trademark, manufacturer, or otherwise, does not necessarily constitute or imply its endorsement, recommendation, or favoring by the United States Govemment or any agency thereof. The views and opinions of authors expressed herein do not necessarily state or refiect those of the United States Govermment or any agency thereof. 


\section{Influence of Prandtl Number and Effects of Disruption Shape on the Performance of Enhanced Tubes with the Separation and Reattachment Mechanism}

by B. Arman and T.J. Rabas

Energy Systems Dirision,

Argonne National Laboratory, 9700 South Cass Avenue, Argonne, Illinois 60439

April 1992

Work sponsored by United States Department of Energy,

Assistant Secretary for Conservation and Renewable Energy, Office of Industrial Technology






\section{Contents}

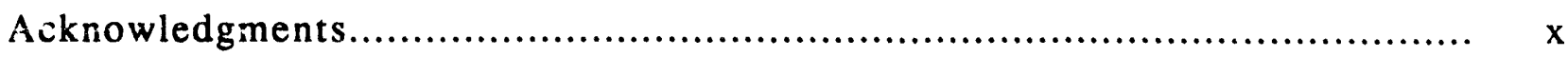



Abstract .............................................................................. 1





2 Separation and Reattachment Mechanism........................................... 8

2.1 Tube Geometries ....................................................... 8

2.1.1 Spirally Indented Tubes................................................. 8

2.1.2 Spirally Ribbed Tubes .................................................. 9

2.1.3 Transverse Repeated-Rib Tubes ....................................... 9

2.2 Disruption-Shape Effects................................................... 10

2.3 Influence of the Prandtl Number ............................................ 16

2.3.1 Savage and Myers ........................................................ 17

2.3.2 Kalinin et al. ............................................................. 17

2.3.3 Webb et al. ......................................................... 19

2.3.4 Berger and Hau ....................................................... 21

2.3.5 Tan and Xaio....................................................... 24

3 Previous Numerical Analysis Investigations ...................................... 26



3.2 Backward-Facing Steps ..................................................... 28

3.3 Sudden Pipe Expansions ................................................ 28

3.4 Enhanced Passages............................................................ 29

3.4.1 Taylor et al. ....................................................... 30

3.4.2 Becker and Rivir....................................................... $\quad 30$

3.4.3 Fodemski and Collins................................................ 31

3.4.4 Hung et al. ................................................................... 32

3.4.5 Hijikata et al. ....................................................... 34

3.4.6 Fujita et al. ........................................................... 34

3.4.7 Barba ................................................................. 35

3.4.8 Patankar et al. ....................................................... 35

4 Governing Equations and Modeling Approach......................................... 36

4.1 Conservation Equations .................................................. 36

4.2 Near-Wall Turbulence Modeling................................................. 41 . 


\section{Contents (Cont.)}

4.2.1 Low-Reynolds-Number Two-Equation Model of

Lam and Bremhorst .................................................. 42

4.2.2 Two-Layer Model of Chen and Patel ................................... 43

4.3 Boundary Conditions ....................................................... 44

4.3.1 Wall and Centerline Boundary Conditions........................... 44

4.3.2 Cyclic Boundary Conditions ........................................ 45

4.4 Turbulent Heat-Transfer Closure ............................................. 48



5.1 Nonorthogonal, Body-Fitted Coordinates.................................. 50

5.2 Transformation of Governing Equations................................... 52

5.3 Numerical Solution Procedure .......................................... 53

5.3 .1 Grid Generation ................................................ 53

5.3.2 Discretization of the Governing Equations............................ 55

5.3.3 Differencing of the Convection Terms................................ 57

5.3.4 Interpolation for Cell-Face Velocities.................................. 61

5.3.5 Pressure-Velocity Coupling.......................................... 62

5.3.6 Solution Procedure...................................................... 64

5.4 Convergence Criteria....................................................... 65

5.4 .1 Grid Dependency.................................................. 65

5.4 .2 Convergence........................................................ 65



6.1 Orthogonal Numerical Code............................................ 67

6.1.1 Comparison of Friction Factors.................................... 67

6.1.2 Comparisons of Mean Heat-Transfer Coefficients ......................... 67

6.1.3 Comparisons of Local Heat-Transfer Coefficients....................... 67

6.2 Body-Fitted Numerical Code............................................. 68

7 Results and Discussion ....................................................... 77

7.1 Influence of the Prandtl Number ......................................... 77

7.1.1 Variation in Local Heat-Transfer Coefficient ............................ 78

7.1.2 Variation in Maximum Enhancement Level at the Rib.................. 81

7.1.3 Influence of the Rib Pitch on the Rib Thermal Performance ........... 85

7.1.4 Thermal Performance in the Downstream Recirculaticn Region.......... 86

7.2 Effects of the Disruption Shape ....................................... 95

7.2.1 Wall Shear Stress and Friction Factors ............................... 95

7.2 .2 Local Pressure Distributions ......................................... 98

7.2.3 Heat-Transfer Enhancement Level.................................... 98

7.2.4 Effect of Pitch................................................... 102 


\section{Contents (Cont.)}

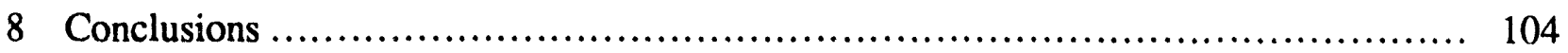

8.1 Influence of the Prandtl Number ............................................ 104

8.2 Effects of the Disruption Shape .......................................... 105



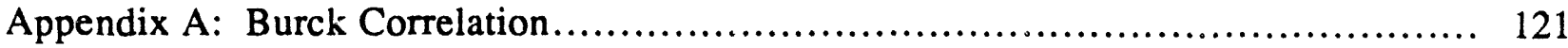



Figures

1.1 Turbulent Flow Structure within an Enhariced Tube with the Separation and Reattachment Mechanism ..................................................... 5

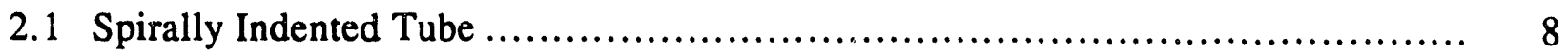

2.2 Disruption Shape Options for the Separation and Reattachment Mechanism....... 10

2.3 Heat-Transfer Performance Variations with Different Disruption Shapes............. 11

2.4 Friction-Factor Variations with Different Disruption Shapes..................... 12

2.5 Friction-Factor and Heat-Transfer Performance Variations with Different Disruption Shapes ........................................................ 13

2.6 Friction-Factor Enhancement-Ratio Variation for Spirally Indented Tubes ......... 14

2.7 Local Heat-Transfer Coefficient Variation with Different Disruption Shapes in a Rectangular Passage ..................................................... 14

2.8 Local Heat-Transfer Coefficient Variation with Different Disruption Shapes



2.9 Efficiency-Index Variation with the Equivalent Sand-Grain Roughness Reynolds Number

2.10 Heat-Transfer Enhancement as a Function of the Roughness Reynolds Number 


\section{Figures (Cont.)}

2.11 Reynolds Number and Prandtl Number Exponents as a Function of the Prandtl Number for the 04/10 Tube Geometry

2.12 Reynolds Number and Prandtl Number Exponents as a Function of the

Prandtl Number for the $01 / 10$ Tube Geometry

2.13 Heat-Transfer Enhancement as a Function of the Roughness

Reynolds Number

3.1 Nusselt Number for the First Heated Rib: $\operatorname{Re}=55,283 \ldots \ldots \ldots \ldots \ldots \ldots \ldots \ldots \ldots$

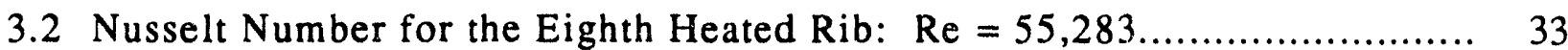

5.1 Staggered and Colocated Grid Layout .................................... 51



5.3 Normalized Variable Diagram for New Composite Scheme ...................... 60

6.1 Comparison of Measured and Predicted Friction Factors as a Function of the Reynolds Number

6.2 Comparison of Measured and Predicted Mean Nusselt Numbers as a Function of the Reynolds Number with the Prandtl Number as a Parameter for the $01 / 10$ Tube.

6.3 Comparison of Measured and Predicted Mean Nusselt Numbers as a Function of the Reynolds Number with the Prandtl Number as a Parameter for the $02 / 10$ Tube

6.4 Comparison of Measured and Predicted Mean Nusselt Numbers as a Function of the Reynolds Number with the Prandtl Number as a Parameter for the $04 / 10$ Tube.

6.5 Comparison of Measured and Predicted Mean Nusselt Numbers as a Function of the Reynolds Number with the Prandtl Number as a Parameter for the $02 / 20$ Tube

6.6 Comparison of Measured and Predicted Mean Nusselt Numbers as a Function of the Reynolds Number with the Prandtl Number as a Parameter for the $02 / 40$ Tube

6.7 Comparison of Measured and Predicted Local Nusselt Numbers as a Function of the Axial Location

6.8 Comparison of Numerically Predicted Friction-Factor and Stanton Number Values with those Obtained with Commonly Used Correlations 


\section{Figures (Cont.)}

6.9 Comparison of the Numerically Predicted Friction Factor with the Measured Values Obtained by Hijikata and Mori for an Arc-Shaped Disruption................

6.10 Comparison of the Numerically Predicted Nusselt Number with the Measured Values Obtained by Hijikata and Mori for an Arc-Shaped Disruption

6.11 Comparison of the Numerically Predicted Friction Factor with the Measured Values Obtained by Nunner for a Semicircular Disruption

6.12 Comparison of the Numerically Predicted Nusselt Number with the Measured Values Obtained by Nunner for a Semicircular Disruption...

7.1 Local Nusselt Number Variation with Axial Distance for the 02/40 Tube with $\operatorname{Pr}=0.71$

7.2 Local Nusselt Number Variation with Axial Distance for the 02/40 Tube with $\operatorname{Pr}=21.7$

7.3 Local Nusselt Number Variation with Axial Distance for the 02/40 Tube with $\operatorname{Re}=6,300$

7.4 Local Nusselt Number Variation with Axial Distance for the 02/40 Tube with $\operatorname{Re}=40,000$

7.5 Maximum Nusselt Number Variation at the Rib for Three Prandtl Numbers and Three Tubes with the $p / e$ Value of 10

7.6 Maximum Enhancement-Level Variation at the Rib as a Function of the Roughness Reynolds Number with the Prandtl Number as a Parameter for the $01 / 10$ Tube Geometry

7.7 Maximum Enhancement-Level Variation at the Rib as a Function of the Roughness Reynolds Number with the Prandtl Number as a Parameter for the $02 / 10$ Tube Geometry

7.8 Maximum Enhancement-Level Variation at the Rib as a Function of the Roughness Reynolds Number with the Prandtl Number as a Parameter for the 04/10 Tube Geometry

7.9 Maximum Enhancement-Level Variation at the Rib as a Function of the Roughness Reynolds Number with the Prandtl Number as a Parameter for the $02 / 20$ Tube Geometry

7.10 Maximum Enhancement-Level Variation at the Rib as a Function of the Roughness Reynolds Number with the Prandtl Number as a Parameter for the 02/40 Tube Geometry 


\section{Figures (Cont.)}

7.11 Maximum Heat Transfer at the Rib as a Function of the Reynolds Number with the Prandtl Number as a Parameter for the 02/10, 02/20, and 02/40 Tube Geometries.

7.12 Magnitudes of the Maximum Heat Transfer in the Downstream Recirculation Region as a Function of the Reynolds Number for $\operatorname{Pr}=0.71, \operatorname{Pr}=21.7$, and the $02 / 10,02 / 20$, and $02 / 40$ Tube Geometries

7.13 Turbulent Kinetic Energy Variation with Distance from the Wall at Various Axial Locations

7.14 Local Wall Shear Stress as a Function of the Axial Location for the 01/10 Tube Geometry

7.15 Local Wall Shear Stress as a Function of the Axial Location for the 02/10 Tube Geometry

7.16 Local Wall Shear Stress as a Function of the Axial Location for the 04/10 Tube Geometry

7.17 Local Wall Shear Stress as a Function of the Axial Location for the 02/20 Tube Geometry

7.18 Local Wall Shear Stress as a Function of the Axial Location for the 02/40 Tube Geometry

7.19 Characteristic Lengths in the Downstream Recirculation Region for the 02/20 Tube Geometry

7.20 Characteristic Lengths in the Downstream Recirculation Region for the 02/40 Tube Geometry

7.21 Characteristic Lengths in the Downstream Recirculation Region for the 01/10 Geometry.

7.22 Characteristic Lengths in the Downstream Recirculation Region for the 02/10 Tube Geometry

7.23 Characteristic Lengths in the Downstream Recirculation Region for the 04/10 Tube Geometry

7.24 Different Disruption Shapes Considered 


\section{Figures (Cont.)}

7.25 Normalized Wall Shear-Stress Distributions for a Sine-Shaped Disruption: $p / e=20$ and $w / e=2$

7.26 Comparison of the Wall Shear-Stress Variations for the Smaller Reynolds

Number: $p / e=20$

7.27 Comparison of the Wall Shear-Stress Variations for the Larger Reynolds Number: $p / \mathrm{e}=20$

7.28 Pressure-Drop Distributions for a Sine-Shaped Disruption: $\operatorname{Re}=9,370$, $p / \mathrm{e}=20$, and $\mathrm{w} / \mathrm{e}=2.0$

7.29 Comparison of the Pressure Distributions for the Smaller Reynolds Number: $p / \mathrm{e}=20$

7.30 Compaiison of the Pressure Distributions for the Larger Reynolds Number: $p / \mathrm{e}=20$

7.31 Heat-Transfer Enhancement Level for a Sine-Shaped Disruption: $p / e=20$ and $w / e=2.0$

7.32 Comparison of the Heat-Transfer Enhancement Levels for the Smaller Reynolds Number: $p / \mathrm{e}=20$ and $\mathrm{e} / \mathrm{D}=0.02$

7.33 Comparison of the Heat-Transfer Enhancement Levels for the Larger Reynolds Number: $p / \mathrm{e}=20$ and $\mathrm{e} / \mathrm{D}=0.02$

\section{Tables}

2.1 Comparison of the Experimental and Predicted Efficiency-Index Values .......... 18

2.2 Tests of Tube Geometries ...................................................... $\quad 20$

5.1 Some Well-Known Discretization Schemes ................................... 58

6.1 Comparison of Measured and Predicted Nusselt Numbers for the Five Tubes Tested by Webb et al.

7.1 Reattachment Lengths and Friction Factors .................................. 98

7.2 Comparison of the Mean Nusselt Numbers .................................. 103 


\section{Acknowledgments}

The authors wish to acknowledge V.C. Patel and F.W. Schmidt for their assistance in overcoming technical hurdles. The authors also thank D. Knoebel, U.S. Department of Energy (DOE), for encouraging us to employ a numerical modeling approach and E.P. HuangFu, also from DOE, for continued support. The authors wish to acknowledge V. Manning, Argonne National Laboratory (ANL), for the initial word-processing support and F. Henning for technical editing.

The report has been reviewed by the following persons:

- D. McEligot EG\&G Idaho, Inc.

- V.C. Patel The University of Iowa

- F.W. Schmidt Pennsylvania State University

- G. Schuz Wieland-Werke AG 


\section{Nomenclature}

$A_{i} \quad$ maximum inside cross-sectional area, $A_{i}=\frac{\pi}{4} D^{2}$

$A_{\varepsilon}$ an empirical constant in algebraic-length scale for turbulent energy dissipation (Equation 4.10a)

$A_{\mu} \quad$ an empirical constant in algebraic-length scale for eddy diffusivity (Equation 4.10b)

$c_{p} \quad$ specific heat

$\mathrm{C}(\mathrm{x}) \quad$ circumference or perimeter of passage

$\mathrm{C}_{\varepsilon 1} \quad$ an empirical constant in $\varepsilon$ equation (Equation 4.6c)

$\mathrm{C}_{\varepsilon 2} \quad$ an empirical constant in $\varepsilon$ equation (Equation $4.6 \mathrm{c}$ )

$\mathrm{C}_{\mu} \quad$ an empirical constant in turbulence momentum eddy-diffusivity equation (Equation 4.6a)

$\mathrm{C}_{l} \quad$ an empirical constant defined by Equation 4.11

D maximum inside pipe diameter

e disruption height

$e_{s g} \quad$ sand-grain disruption height

$\mathrm{e}^{+} \quad$ roughness Reynolds number, $\mathrm{e}^{+}=(\mathrm{e} / \mathrm{D}) \operatorname{Re} \sqrt{\mathrm{f} / 2}$

$\mathrm{e}_{\mathrm{sg}}^{+} \quad$ sand-grain roughness Reynolds number, $\mathrm{e}_{\mathrm{sg}}^{+}=\left(\mathrm{e}_{\mathrm{sg}} / \mathrm{D}\right) \mathrm{R}_{\mathrm{e}} \sqrt{\mathrm{f} / 2}$

E an empirical constant in the momentum wall-function expression (Equation 3.1)

E' an empirical constant in the temperature wall-function expression (Equation 3.2)

$\mathrm{f}$ enhanced tube friction factor, $\mathrm{f}=\Delta \mathrm{p} /\left(0.5 \rho \overline{\mathrm{U}}^{2}\right)$

$f_{p} \quad$ plain tube friction factor

$\mathrm{f}_{\varepsilon 1} \quad$ wall-damping coefficient defined by Equation $4.7 \mathrm{~b}$

$f_{\varepsilon 2} \quad$ wall-damping coefficient defined by Equation $4.7 \mathrm{c}$

$\mathrm{f}_{\mu} \quad$ wall-damping coefficient defined by Equation $4.7 \mathrm{a}$

G turbulent kinetic energy generation defined by Equation $4.6 \mathrm{c}$

$\mathrm{h}$ enhanced tube mean (spatial average) heat-transfer coefficient

$h_{\max } \quad$ enhanced tube maximum local heat-transfer coefficient

$h_{p} \quad$ plain tube mean (spatial average) heat-transfer coefficient

$h_{x} \quad$ enhanced tube local heat-transfer ccefficient

$\mathrm{k} \quad$ turbulent kinetic energy defined by Equation $4.4 \mathrm{~g}$

$k \quad$ thermal conductivity

$l_{\alpha} \quad$ length scale

$l_{\mu} \quad$ length scale for viscosity

$l_{\varepsilon} \quad$ length scale for dissipation 


\begin{tabular}{|c|c|}
\hline m & Prandtl number exponent \\
\hline$\dot{\mathbf{m}}$ & mass flow rate \\
\hline n & number of flutes, or normal distance, or the Reynolds number exponent \\
\hline $\mathrm{Nu}$ & mean (spatial average) Nusselt number, $\mathrm{Nu}=h \mathrm{D} / \mathrm{k}$ \\
\hline $\mathrm{Nu}_{\max }$ & maximum local Nusselt number value, $N u_{m}=h_{\max } D / k$ \\
\hline $\mathrm{Nu}_{\mathrm{p}}$ & mean (spatial average) Nusselt number for plain tube, $N u_{p}=h_{p} D / k$ \\
\hline $\mathrm{Nu}_{\mathbf{x}}$ & local Nusselt number, $\mathrm{Nu}_{\mathrm{x}}=\mathrm{h}_{\mathrm{x}} \mathrm{D} / k$ \\
\hline$p$ & disruption pitch \\
\hline p & time-averaged pressure \\
\hline $\mathbf{p}^{\prime}$ & fluctuating pressure \\
\hline $\mathbf{P}$ & $\begin{array}{l}\text { time-averaged periodic pressure defined by Equation } 4.20 \text {, or control-volume } \\
\text { center point }\end{array}$ \\
\hline$P$ & instantaneous pressure \\
\hline $\operatorname{Pr}$ & Prandtl number \\
\hline $\operatorname{Pr}_{\mathrm{t}}$ & turbulent Prandtl number \\
\hline$\dot{\mathrm{q}}_{\mathrm{w}}$ & wall heat flux \\
\hline Q & total heat transferred for one pitch length defined by Equation 4.28 \\
\hline $\mathbf{r}$ & radial coordinate \\
\hline $\mathbf{R}$ & pipe radius \\
\hline $\operatorname{Re}$ & Reynolds number, $\operatorname{Re}=\frac{\rho D \bar{U}}{\mu}$ \\
\hline $\mathbf{R e}_{\mathbf{t}}$ & Reynolds number defined by Equation $4.8 \mathrm{~b}$ \\
\hline $\mathbf{R e}_{\mathbf{y}}$ & Reynolds number defined by Equation $4.8 \mathrm{a}$ \\
\hline $\mathbf{S}$ & source term \\
\hline St & mean (spatial average) Stanton number, $\mathrm{St}=\mathrm{Nu} /(\mathrm{RePr})$ \\
\hline $\mathrm{St}_{\mathrm{p}}$ & mean (spatial average) Stanton number for plain tube, $S t_{p}=N u_{p} /(\operatorname{RePr})$ \\
\hline $\mathbf{t}$ & time \\
\hline $\mathrm{T}$ & time-averaged temperature \\
\hline$T$ & instantaneous temperature \\
\hline$T^{\prime}$ & fluctuating temperature \\
\hline $\mathrm{T}^{+}$ & dimensionless temperature, $T^{+}=\frac{\rho c_{p}\left(T_{w}-T\right) U^{*}}{\dot{q}_{w}}$ \\
\hline $\mathrm{T}_{\text {ref }}$ & reference temperature \\
\hline $\mathbf{u}$ & time-averaged axial velocity component \\
\hline $\mathbf{u}^{\prime}$ & fluctuating axial velocity component \\
\hline $\mathbf{u}^{+}$ & dimensionless axial velocity, $\mathrm{u}^{+}=\mathfrak{u} / \mathrm{U}^{*}$ \\
\hline $\mathrm{U}$ & instantaneous axial velocity component \\
\hline
\end{tabular}




$\begin{array}{ll}U_{\alpha} & \text { local velocity } \\ U_{c} & \text { velocity defined by Equation } 5.5 a \\ U^{*} & \text { friction velocity, } U^{*}=\left(\tau_{w} / \rho\right)^{1 / 2} \\ \bar{U} & \text { mean velocity, } \bar{U}=\dot{m} / \rho A_{i} \\ v & \text { time-averaged radial velocity component } \\ v^{\prime} & \text { fluctuating radial velocity component } \\ V & \text { instantaneous radial velocity component } \\ V_{c} & \text { velocity defined by Equation } 5.5 b \\ w & \text { disruption width or time-averaged angular velocity component } \\ w^{\prime} & \text { fluctuating angular velocity component } \\ W^{\prime} & \text { instantaneous angular velocity component } \\ x & \text { axial coordinate } \\ y & \text { distance from the wall } \\ y^{+} & \text {dimensionless distance from wall, } y^{+}=\frac{y U^{*}}{v}\end{array}$

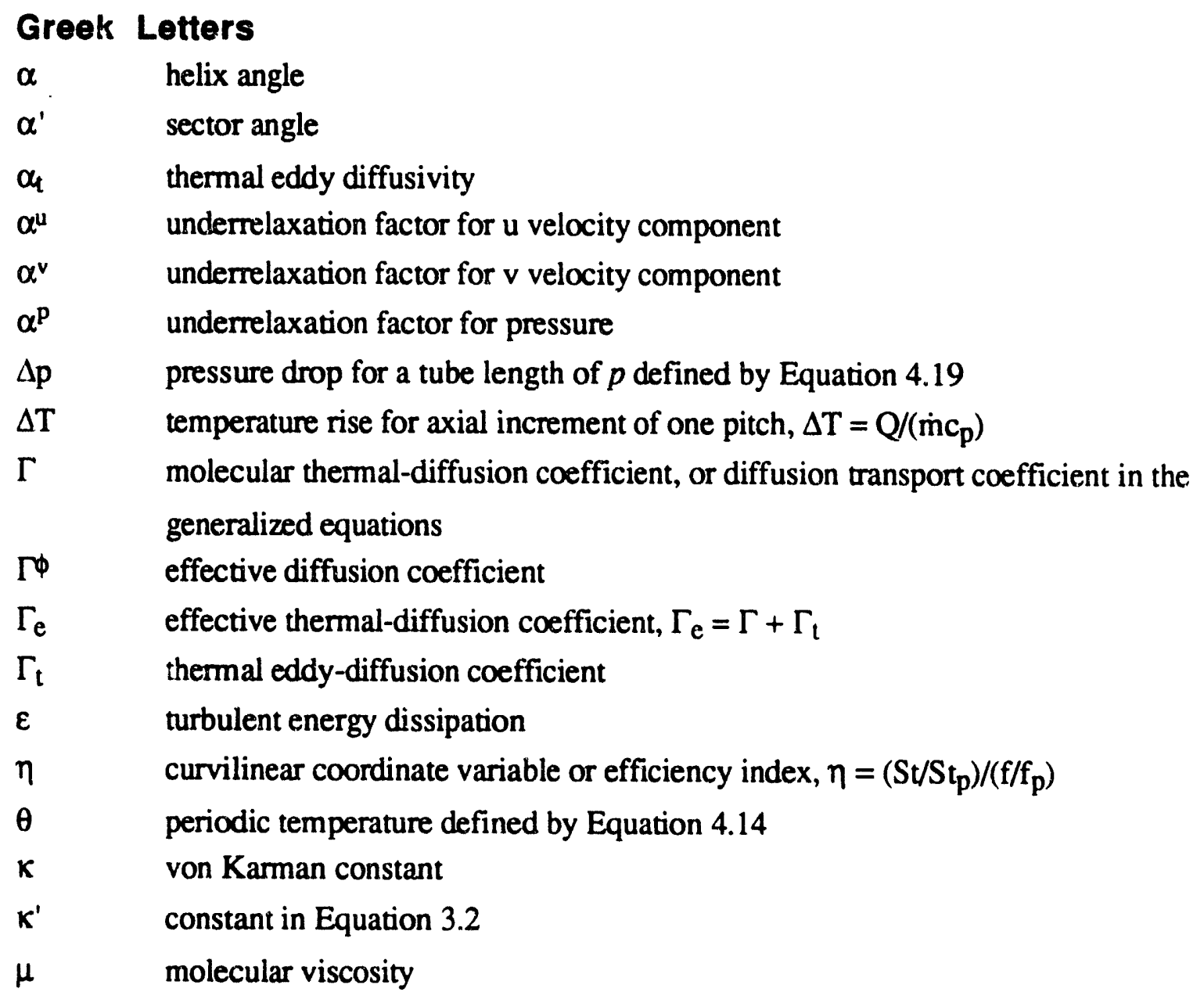




$\begin{array}{ll}\mu & \text { molecular viscosity } \\ \mu_{\mathrm{e}} & \text { effective viscosity, } \mu_{\mathrm{e}}=\mu+\mu_{\mathrm{t}} \\ \mu_{\mathrm{t}} & \text { turbulent viscosity } \\ v & \text { molecular diffusivity } \\ v_{\mathrm{t}} & \text { eddy diffusivity, } v_{t}=\mu_{\mathrm{t}} \rho \\ \xi & \text { curvilinear coordinate variable } \\ \rho & \text { density } \\ \sigma_{\varepsilon} & \text { empirical constant in } \varepsilon \text { equation } \\ \sigma_{\mathrm{k}} & \text { empirical constant in } \mathrm{k} \text { equation } \\ \tau_{\mathrm{w}} & \text { wall shear stress } \\ \phi & \text { arbitrary variable } \\ \Phi & \text { normalized variable } \\ \nabla 2 & \text { Laplacian operator in the orthogonal coordinates }\end{array}$




\title{
Influence of Prandtl Number and Effects of Disruption Shape on the Performance of Enhanced Tubes with the Separation and Reattachment Mechanism
}

by

\author{
B. Arman and T.J. Rabas
}

\begin{abstract}
The pressure-drop and heat-transfer performance of an enhanced tube with transverse disruptions can be predicted with a numerical modeling method, an accomplishment not previously achieved. Two computer codes wer: employed to achieve this goal - an orthogonal code and a nonorthogonal, body-fitted code. The turbulence closure was achieved with a two-layer turbulence model. The orthogonal computer corle was used to determine the influence of the Prandtl number. The numerical simulations demonstrated that six distinct regions exist and that three zones dominate the thermal performance. The nonorthogonal, body-fitted numerical code was used to determine the thermohydraulic performance of enhanced tubes with transverse, periodic sine-, semicircle-, arc-, and trapezoidshaped disruptions. The research showed that there was a trade-off between the heat-transfer and pressure-drop performances when the disruption shape becomes more contoured, and that the local heat transfer is strongly dependent on the shape in the vicinity of the disruption, but it is less dependent in the downstream recirculation region and in the boundary-layer development zone.
\end{abstract}

\section{Summary}

Numerical analysis was successfully used to evaluate the single-phase thermal-hydraulic performance of internally enhanced heat-exchanger tubes. A two-dimensional, axisymmetric flow field was selected because it is an accurate representation for the internal geometry considered in this investigation (two-dimensional transverse disruptions or ribs). The flow field was considered to be fully developed because the tube length is much larger than the tube diameter for typical heat exchangers. Both laminar and turbulent flow were considered, although the work discussed in this report focuses on the more important turbulent case.

Very good agreement was obtained between the predicted friction factors and mean heattransfer coefficients and the corresponding measured values. For this validation, five tube geometries with rectangular disruptions (Webb et al. 1971), two tube geometries with arc-shaped disruptions (Hijikata and Mori 1987), and one tube with a semicircular disruption (Nunner 1956) 
were used. Average and maximum errors of $15 \%$ and $31 \%$, respectively, were obtained in comparisons of the mean heat-transfer coefficients for all five tube geometries and four different Prandtl number fluids considered by Webb et al. (1971).

The influence of the Prandtl number, Pr, on the thermal performance for flow inside a tube with transverse disruptions is very different from that for a smooth tube. The flow field in the former case contains separations and reattachments at the disruption and in the downstream nsattachment region. The thermal-performance characteristics of separating and reattaching flows are very different from those of boundary-layer flows, although the boundary-layer concepts are still used in an attempt to explain many of the features of single-phase flow inside enhanced passages.

The effect of the Prandtl number on the thermal performance is most easily understood by observing how the local heat-transfer-coefficient variation is changed at and between two adjacent disruptions. There are two maximums in the thermal performance: one at the top surface of the disruption and another in the downstream recirculation region near the reattachment point. In the vicinity of the disruption, an insease in Pr leads to a substantial thermal-performance increase, and this contribution dominates at high Pr values. The magnitude of the maximum heat-transfer coefficient at the rib follows a pattern of attaching or impinging flows with exponents on the Reynolds and Prandtl numbers of about 0.65 and 0.33 , respectively. The contributions from the regions.between two adjacent disruptions - the recirculation, boundary-layer development, and the upstream recirculation - are less dependent on Pr. The magnitude of the maximum values follows a pattern similar to that at the disruption because of the attaching or impinging flow field. The location of the maximum heat-transfer coefficient in this region moves upstream, toward the disruption, with increasing Prandtl and Reynolds numbers and is bounded by the locations of the maximum wall shear stress and the reattachment point. The major reason for the heat-transfer enhancement in the downstream recirculation region is the high turbulence levels near the surface.

Comparisons of the friction factors and heat-transfer coefficients were made between four different disruption shapes: sine, semicircular, arc, and trapezoidal. The difference in the friction factor values for the four shapes was not large (about 14\%), and this difference increased with increasing Reynolds number. The friction factors were the largest for the sine and trapezoidal shapes and smallest for the arc shape. The difference in the heat-transfer-coefficient values for the four shapes was also about $14 \%$, but this difference decreased with increasing Reynolds number. Also, the heat-transfer results for the sine shape were lower than those for the trapezoidal shape.

The impact of the shape on the thermal-hydraulic performance is concentrated in the region of the disruption, and it has almost no effect on the performance in the boundary-layer development region. As a result, the effect of the shape on the thermal-hydraulic performance decreases as the pitch or spacing increases. If the pitch-to-height ratio exceeds about 20 , the effect of the shape can be neglected. The disruption shape does influence the reattachment length; the longer the reattachment length, the higher the friction factors and heat-transfer coefficients. 


\section{Introduction}

Improved heat-transfer performance is a goal that continues to be pursued for many heatexchange applications. Important examples are recuperative and regenerative heat exchangers, gasturbine blade cooling, and cooling of electronic components. The obvious advantages of improved thermal performance are a reduction of energy consumption and/or the reduction of the component size and cost. For the case of heat exchangers, estimated energy savings of about 2.: Quads* per year can be achieved through the use of enhanced surfaces (Rebello 1987). Improved gas-turbine blade cooling results in higher gas temperatures and, therefore, higher efficiencies. Improved electronic equipment can increase computing speed and reduce size and cost.

The most mature and widely applied enhancement method is the use of surface modifications that $\boldsymbol{P}_{i} \mathrm{e}$ commonly called enhanced or augmented surfaces. The measured heattransfer performance with enhanced surfaces can be greater than that of plain surfaces by a factor of three or more. Herein is the motivation for the use of this passive enhancement technique. Many enhanced surfaces have been proposed and used for both single-phase and two-phase applications. Some commonly used enhancement techniques are extended or finned passages; two-dimensional ribs, indentations, or flutes; three-dimensional roughness or coatings; and wire or twisted-tape inserts.

An enhanced surface increases the heat-transfer rate between a solid surface and a fluid and thereby decreases the heat-exchanger size and/or decreases the required ternperature difference. Enhancements are commonly used for some applications but are rejected for others. For about 100 years, finning was used to improve the performance of automotive radiators, and wire inserts were used to enhance the performance of boiler fire tubes. Enhancements are almost always used when one of the heat-exchange fluids (such as air and mosi other gases) has considerably poorer heattransfer characteristics than the other. Enhancements are also used for other reasons, including those listed below:

- The need for modern, efficient technology in a very competitive marketplace, such as in the air-conditioning and refrigeration industries;

- The need for compactness, such as in the automotive and aircraft industries; and

- The overriding need to deal with high heat fluxes associated with the cooling of gas-turbine blades, electronic components, and targets of intense X-ray beams.

* 1 Quad = $10^{15}$ Btu. 
In almost every industry, both enhanced and unenhanced heat exchangers are used. Listed below, by priority, are the major reasons for not using enhancements:

- Cost-to-benefit ratios are insufficient,

- Established prediction methods (performance liability concerns) are lacking,

- Fouling is of concern,

- Tubes made from the desired material and/or with the desired geometries are not available,

- Long-term performance is not clearly demonstrated,

- End user is reluctant to change,

- High thermal-performance levels already exist, and

- Suppliers are not aggressively promoting the change.

Empirical data obtained with commercially available tubing are the basis for the design of heat exchangers when enhanced surfaces are used today. As a result, the designer is limited to only a few surface types made by a limited number of tubing manufacturers: Yorkshire Alloys, Ltd. (1982); Wolverine Tube, Inc. (Wolverine Engineering Data Book II 1984); Hitachi Cable, Ltd. (1987); and Wieland-Werke AG (1989). Currently, the designer does not have access to high-quality prediction techniques that permit the selection of the enhanced-surface geometry to optimize a process and/or system. The lack of high-quality predictions is, therefore, an obvious deterrent to the use of enhanced surfaces.

The major users of enhanced surfaces, such as the air-to-air refrigeration industry, commonly deal with fluids with nonfouling characteristics. However, fouling is commonly encountered with applications where river water, cooling-tower water, and bay and seawater are used as coolants. A very clear pattern exists: if there is a potential fouling problem, there is a reluctance to use enhanced surfaces. Although all of the reasons listed above should be addressed, particular emphasis should be placed on the need to obtain reliable prediction techniques and to develop the knowledge to mitigate and/or control fouling.

An active research task at Argonne National Laboratory (ANL) is the development of a technology base - the prediction capability and fouling mitigation and control - for enhanced surfaces. This activity, which is sponsored by the Thermal Sciences Program of the Advanced Industrial Concepts Division of the U.S. Department of Energy, is currently focused on singlephase enhanced surfaces inside passages. The purpose of this research is to develop reliable 
prediction methods for applications where enhanced surfaces are already used and to overcome the second major barrier to their acceptance. In addition, a fundamental understanding of the basic flow and heat-transfer phenomena will be obtained. The focus will switch to two-phase enhanced surfaces upon completion of the single-phase effort.

The research for improving the single-phase prediction capability deals with consolidating the existing data, filling voids where they exist, and developing high-quality mechanistic prediction methods for the most energy-efficient mechanisms. Prediction methods based on the controlling mechanism are desired because they permit extrapolations into untested regions. This capability is needed to obtain optimized geometries for each end-user application.

Figure 1.1 is a sketch of the surface geometry characteristic of the enhancement mechanism considered in this report. A square disruption shape is shown, but the actual shape of the disruptions of commercial tubing can be quite different. Note that the disruptions are transverse to the flow ( $90^{\circ}$ helix angle) ${ }^{*}$ and are separated by a distance or pitch that exceeds the reattachment length. Three zones exist between the disruptions: the main recirculation zone, the boundary-layer development zone, and the upstream recirculation zone. This enhancement mechanism is called separation and reattachment (Rabas 1989).

The purpose of the work described in this report is to determine the effects of the disruption shape and the Prandtl number on the thermal performance for this enhancement mechanism. Resolving these uncertainties will permit the development of the needed mechanistic prediction method for this high-ranking enhancement mechanism (Rabas 1989).

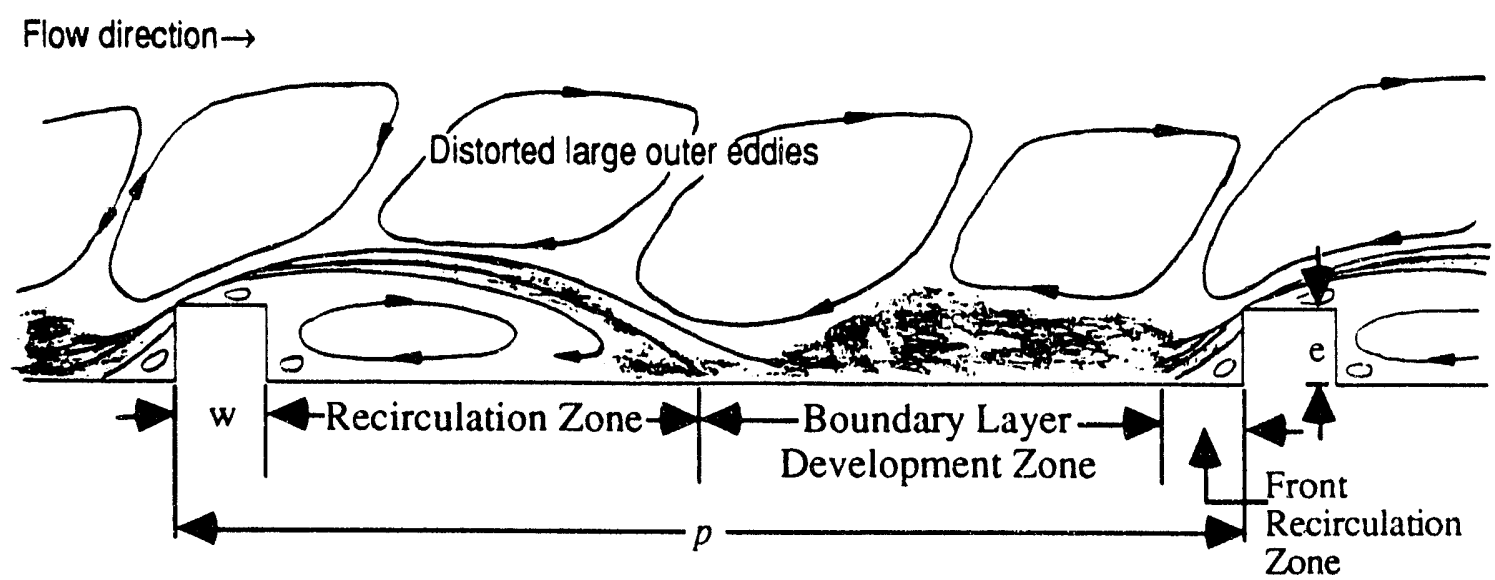

FIGURE 1.1 Turbulent Flow Structure within an Enhanced Tube with the Separation and Reattachment Mechanism

\footnotetext{
* The helix angle is defined as the angle between the centerline of the tube and the disruption. The helix angles of transverse disruptions and longitudinal disruptions are $90^{\circ}$ and $0^{\circ}$, respectively.
} 
A numerical approach was selected to develop the single-phase prediction method for enhanced tubes with the separation and reattachment mechanism. Significant progress has been made in the past 10 years in the numerical modeling of flows inside passages with steps, cavities, and obstructions. The major reasons for this progress are new turbulence models and improvements in computer hardware capabilities. The researchers have focused on simple flows in order to obtain a fundamental understanding of the basic fluid mechanics. Of particular interest were the closure methods for the momentum equations and the turbulence characteristics in the near-wall region. An extensive database of experimental results existed for validation of the numerical predictions. This understanding of the closure methods and the turbulence characteristics is essential to obtain reasonable heat-transfer-coefficient predictions with numerical methods. A review of the literature revealed an increasing number of studies devoted to the prediction of turbulent heat transfer obtained with enhanced passages. Almost all of the studies were concerned with developing flows and/or single disruptions rather than a fully developed flow field with multiple disruptions, as presumably exists with typical enhanced-tube applications.

Section 2 of this report first presents the tube geometries that fall into the category of the separation and reattachment enhancement mechanism: spirally ribbed, spirally indented, and transverse ribs. Comparisons of data with different disruption shapes are then presented to demonstrate that there can be significant differences in both the pressure-drop and heat-transfer performance. There are, however, no prediction methods that are capable of properly accounting for the different shapes. By using existing experimental data and prediction methods, it is clearly demonstrated that the influence of the Prandtl number on the thermal performance is far from being understood. A fundamental understanding of the Prandtl number's effect is essential for the development of the desired mechanistic prediction methods.

Section 3 reviews the progress of related efforts in numerical modeling research. Concepts considered in this review include backward-facing steps and sudden pipe expansions, as well as fully developed flow in enhanced passages because of the common features of a separation followed by a reattachment. Eight numerical-analysis investigations devoted to heat-transfer predictions in enhanced passages were discovered; however, there was no investigation dealing with fully developed turbulent flow inside a circular passage with transverse disruptions.

Section 4 presents the governing equations and the modeling approach that were selected and used. The governing equations include the continuity, momentum, and energy equations. The $\mathrm{k}$ and $\varepsilon$ equations are used for closure in the bulk of the flow field. For the $\mathrm{k}-\varepsilon$ closure in the nearwall region, both the low-Reynolds-number two-equation model of Lam and Bremhorst (1988) and the two-layer model of Chen and Patel (1988) were considered, and the latter was finally selected. The boundary conditions at the wall are no-slip velocity values and constant heat flux, whereas the centerline boundary conditions are based on the symmetry condition - zero radial gradients. Periodic boundary conditions are needed for the velocity, pressure, and temperature fields because of the fully developed flow condition. This condition existed in almost all heatexchanger applications because of the long tube length with respect to the tube diameter. The selected procedures for dealing with the periodicity conditions were developed by Patankar et al. (1977). The expression developed by Malhotra and Kang (1984) was selected for closure of the energy equation - the selection of an expression for the turbulent Prandtl number. 
Section 5 presents the numerical approach. During this study, two different numerical codes were employed: an orthogonal code and a nonorthogonal body-fitted code. A brief summary of the orthogonal code is given, and the methodology for the body-fitted code is discussed. The nonorthogonal curvilinear coordinates and their advantages over the orthogonal coordinates are also discussed. The governing equations are transformed into the new coordinates while the Cartesian velocities are kept as the dependent variables. Other topics discussed include the nonorthogonal body-fitted grid generation procedure (accomplished by means of elliptic differential equations), the discretization of the governing equations in a nonstaggered grid system, convective-term differentiation and cell-face velocity interpolation, the algorithm for the pressurevelocity coupling and the overall solution procedure, and the convergence criterion used during the research.

Section 6 compares the numerically predicted results with experimental data. For rectangular disruption shapes (the orthogonal program is used), the extensive database of Webb et al. (1970) was used for validation of the friction-factor and mean heat-transfer-coefficient predictions, and the data of Baughn and Roby (1992) were used for the validation of the local heattransfer coefficients. Good agreement was obtained. In general, the numerical method underpredicted the experimental results, with the maximum errors occurring in the high-Reynoldsnumber ( $R e$ ) range but never exceeding about $30 \%$. For nonrectangular disruption shapes (the body-fitted numerical program is used), the data of Hijikata and Mori (1987) and Nunner (1956) were used to validate the friction-factor and mean heat-transfer-coefficient predictions. Again, good agreement was obtained.

Section 7 describes the influence of the Prandtl number and the disruption shape on the performance of enhanced tubes with the separation and reattachment mechanism. Section 8 summarizes these findings. 


\section{Separation and Reattachment Mechanism}

The purpose of this research is to obtain an understanding of how the shape of the surface disruption and the Prandtl number influence the performance characteristics of single-phase flow inside circular passages (heat-exchanger tubes) for a particular enhancement mechanism. This enhancement mechanism, called separation and reattachment, is one with transverse or neartransverse disruptions (helix angle greater than about $70^{\circ}$ ) with pitch-to-height ratios greater than about six (Figure 1.1). For this mechanism, a two-dimensional, axisymmetric flow field exists, rather than the more general three-dimensional case with intermediate helix angles.

\subsection{Tube Geometries}

Tubes that fall into the separation-and-reattachment mechanism category are commonly used for single-phase tube-side applications and have practical importance to the heat-exchanger industry. Rabas (1989) has demonstrated that this mechanism ranks high with respect to enhancement level and energy efficiency and ranks low with respect to fouling propensity, and that it is inferior to only the swirl-induced mechanisms. The categories of tubes with the separation and reattachment mechanism include the entire family of single-start spirally indented tubes, single-start spirally ribbed tubes, and transverse repeated-ribbed tubes. Each of these tubes is briefly described in the following sections.

\subsubsection{Spirally Indented Tubes}

A particular example of a spirally indented tube is shown in Figure 2.1. Other names for these tubes are corrugated or rope tubes. These tubes have been proposed for use (Withers and Young 1971a, 1971b; Webb et al. 1984; Mussalli and Gordon 1984; Nikolaev et al. 1982; Boyd et al. 1983; Katsman and Kishinevsky 1983; Rabas et al. 1990) in surface condensers and feedwater heaters in the power industry. These tubes have also been used in the desalination industry (Houle and Buhrig 1973; Inoue et al. 1975). The pitch-to-height ratio restriction is made

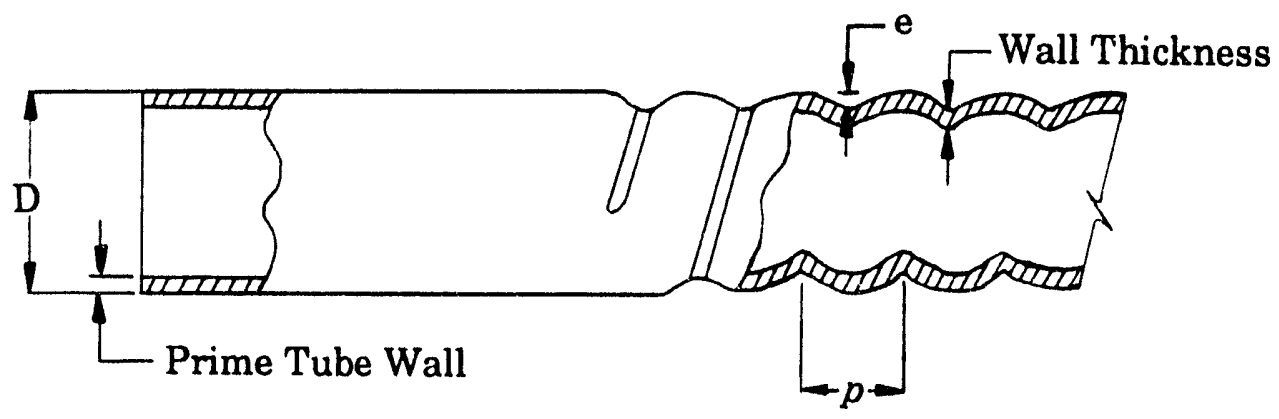

FIGURE 2.1 Spirally Indented Tube 
for practical considerations, such as manufacturing ease and, more importantly, fouling considerations. A wavy disruption shape (Figure 2.1) is the most common shape. An extensive database exists for these tube types, as is evident from the publication of Ravigururajan and Bergliss (1986b).

Experiments and applications with a limited number of these surfaces conducted by Kornbau et al. (1983), Rowe (1983), Panchal (1989), Panchal and France (1992), and Rabas et al. (1990, 1991) suggest that the fouling rate is similar to that of plain tubes. In addition, Eimer (1982), Renfftlen (1991), and Webb and Chamra (1991) have demonstrated that these tubes can be effectively cleaned with the ball-cleaning process when the groove pitch exceeds about $4 \mathrm{~mm}$. Finally, rope tubes are one of the most cost-effective enhanced surfaces (Withers 1980). Because of the practical importance of spirally indented tubes, there is ample motivation to further understand the details of the separation and reattachment mechanism. More detailed information on spirally indented tubes is given by Newson (1976), Hill (1978), and Webb (1982).

\subsubsection{Spirally Ribbed Tubes}

Another family of tubes with near-transverse disruptions or ribs can be made with soft materials such as copper, copper-based alloys, and aluminum by an extrusion process. This manufacturing process is commonly used to make intermediate helix angles, but it can also be used to gentrate the geometry required for the separation and reattachment mechanism (Hitachi 1987). These tubes are commonly used in the refrigeration and air-conditioning industries and have been studied by Nakayama et al. (1983). The disruption shapes are usually semicircular when made by this manufacturing process, as shown in the top portion of Figure 2.2.

\subsubsection{Transverse Repeated-Rib Tubes}

Another enhancement type that falls into the separation and reattachment category is the transverse repeated rib - ribs with a $90^{\circ}$ helix angle. This enhancement type has become a benchmark for performance evaluation and comparison. Transverse repeated ribs with square or rectangular shapes have received attention because of their simple geometry, which lends itself to analysis. However, transverse repeated ribs are not commercially viable because of manufacturing difficulties. High-quality experimental data have been obtained by Webb et al. (1971), Berger and Whitehead (1977), Berger and Hau (1979), Mendes and Mauricio (1987), and Baughn and Roby (1992). Also, Kalinin et al. (1970) have compiled an extensive data bank for tubes with transverse repeated ribs that were made by a grooving process. These databases will be used to "tune" the numerical prediction method presented in this report. 


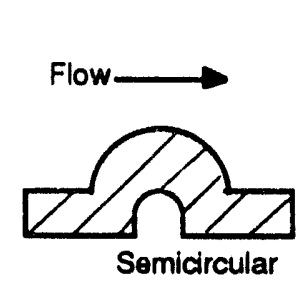

Shapes for Spirally Indented Tubes
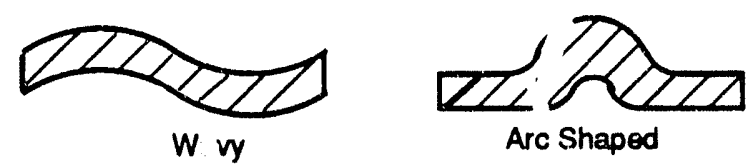

Arc Shaped

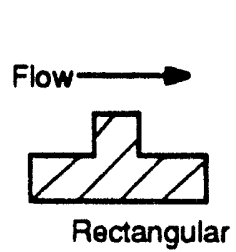

Other Possible Disruption Shapes
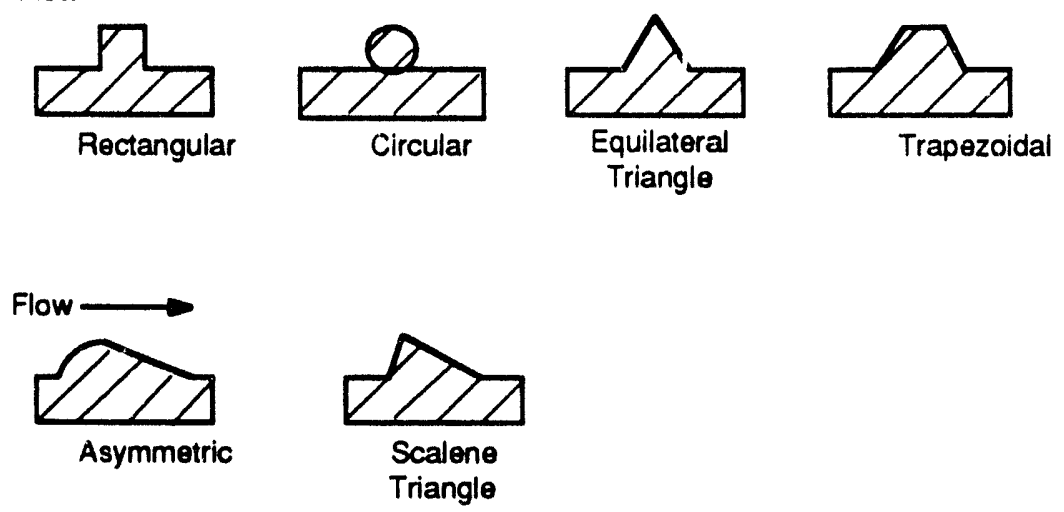

FIGURE 2.2 Disruption Shape Options for the Separation and Reattachment Mechanism

\subsection{Disruption-Shape Effects}

The parameters that control the thermal-hydraulic performance of tubes with the separation and reattachment mechanism are the disruption height, pitch, and shape. The width of the disruption is not considered as an independent parameter but as part of the shape effect. Figure 1.1 shows a sketch of a square disruption, and Figure 2.2 shows some different disruption shapes. For commercially available, spirally indented tubes, the three common shapes are semicircular, wavy, and arc-shaped. The tubes made by most manufacturers - Yorkshire Alloys, Ltd. (1982), Wolverine Tube, Inc. (Wolverine Engineering Data Book II 1984), Hitachi Cables, Ltd. (1987), and Wieland-Werke AG (1989) - are the wavy type, whereas the tubes tested by Ganeshan and Raja Rao (1982) and Sethumadhavan and Raja Rao (1983) were semicircular. Tubes with an arcshaped disruption are easily manufactured by roll-pressing (Hijikata et al. 1987; Hijikata and Mori 1987) and have been tested by $\mathrm{Li}$ et al. (1982). In general, a wide range of disruption sizes, pitches, and shapes can be obtained; the major limitations are the manufacturing method and/or the tubing material. Although the disruption shape is an important parameter for predicting performance, it is not included in most existing prediction methods.

The effects of the height and pitch of the disruption have been adequately determined by experiments, and it has been conclusively demonstrated that these geometry characteristics are important correlation parameters. As a result, the disruption height (e) and pitch $(p)$ are necessary parameters in all of the recommended prediction methods for tubes with the separation and 
reattachment enhancement mechanism. The heat transfer and pressure drop increase when the disruption height increases, and both decrease when the disruption pitch increases. Many empirical prediction methods do an adequate job to account for these effects, provided that all of the tubes used to generate the experimental database have been made by the same manufacturing process (giving essentially the same disruption shape), and that the same fluid (e.g., air, water, etc.) has been used. Typical examples are the correlations of Kalinin et al. (1970), Mehta and Raja Rao (1979), Withers (1980), Li et al. (1982), and Nakayama et al. (1983). However, these empirical correlations yield unacceptable results for geometries that were not considered in their development. Panchal and France (1992) have clearly demonstrated this point for spirally indented tubes with a large pitch.

Figures 2.3 and 2.4 present a comparison of the heat-transfer and friction-factor values obtained by Nunner (1956) with four different tubes. To the authors' knowledge, Nunner (1956) is the first researcher to have attempted to determine the effect of the rib shape and publish the results. All four tubes have the same disruption height but two different $p / e$ ratios and three different shapes. The test fluid was air. For a fixed $p / e$, note that both the friction-factor and Nusselt number $(\mathrm{Nu})$ values decrease with the disruption curvature; however, the reductions of the heat-transfer values are less than the reductions in measured friction-factor values.

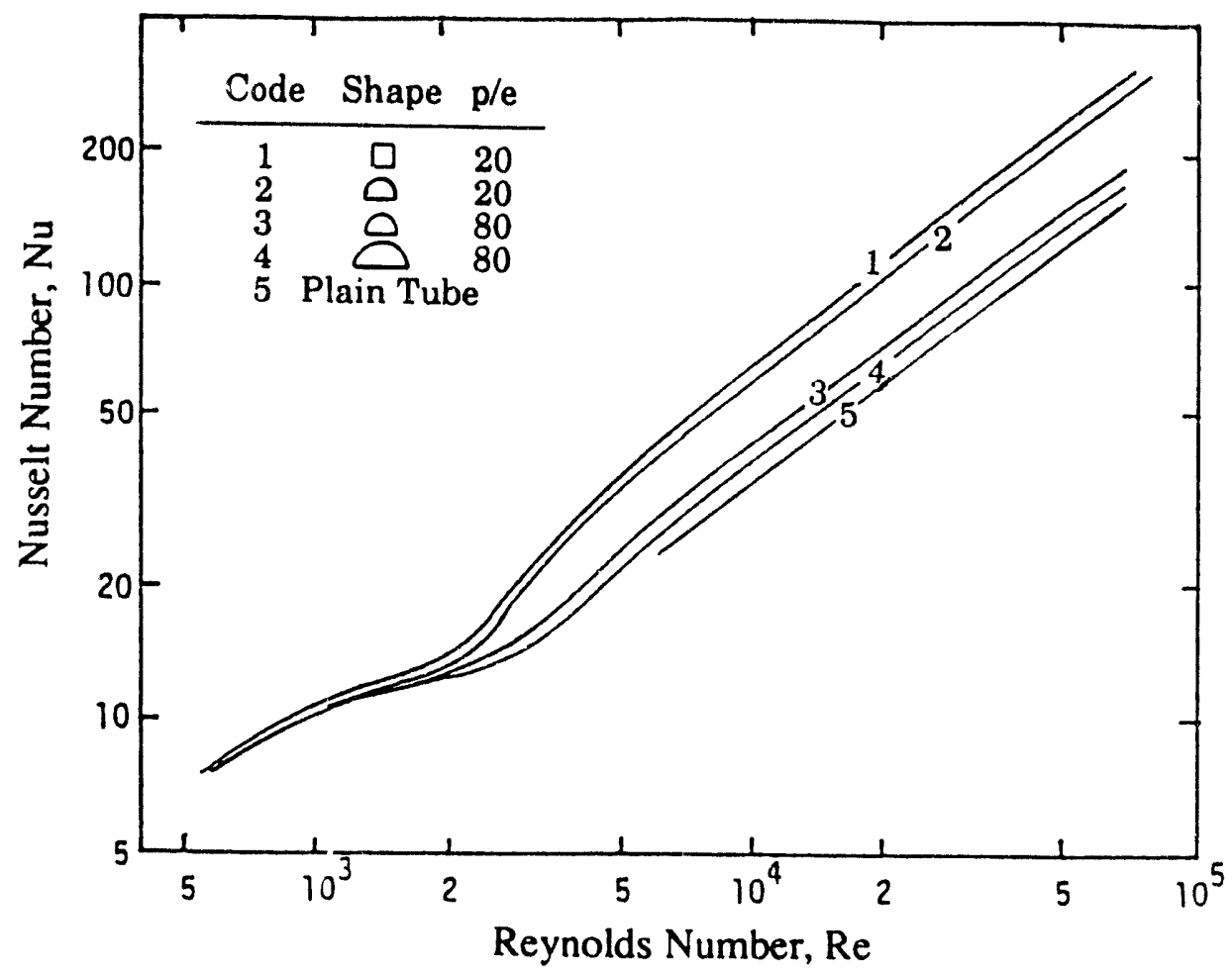

FIGURE 2.3 Heat-Transfer Performance Variations with Different Disruption Shapes (Source: Nunner 1956) 


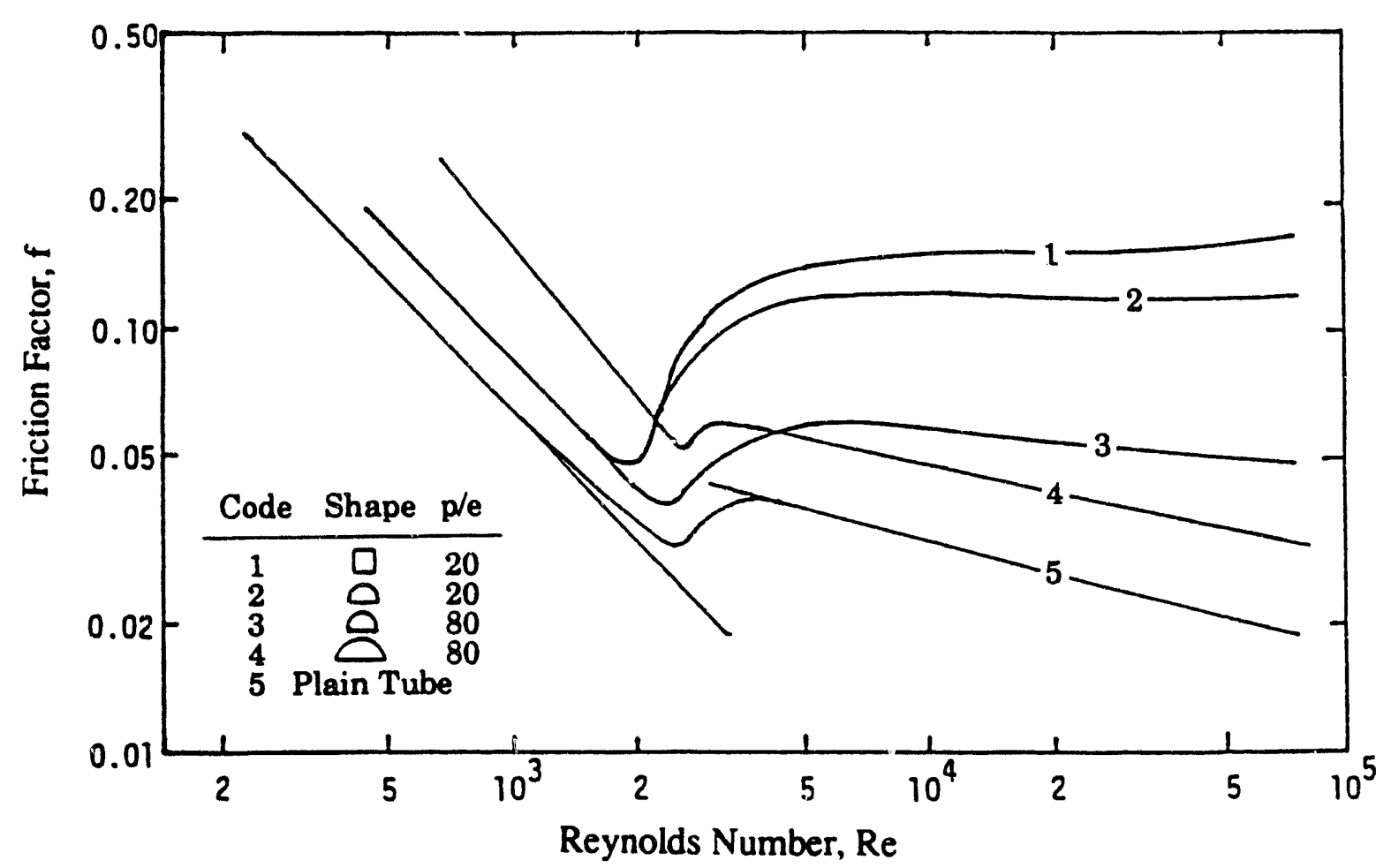

FIGURE 2.4 Friction-Factor Variatio.1s with Different Disruption Shapes (Source: Nunner 1956)

Figure 2.5 presents a similar comparison of the mean Stanton number $(\mathrm{St})$ and frictionfactor $(f)$ values for the same disruption height and pitch, but for different shapes (obtained for the experimental work of Han et al. 1978). Air was used as the test fluid. Again, note that the shape causes a significant variation in the friction-factor values but a smaller variation in the thermal performance.

Even for tubes of the same family, such as spirally indented or rope tubes, the disruption shape affects the pressure-drop prediction. Figure 2.6, taken from Rabas et al. (1988), shows that the friction-factor enhancement ratios at $R e=\$ 0,000$ are a function of the severity ratio, defined as $e^{2} /(p D)$, where $D$ is the pipe diameter, for 60 different tubes from eight different sources. Note that the data fall into three groupings on the basis of the manufacturer and/or shape resulting from the indenting process: wavy (Young et al. 1968, 1969, 1975; Withers 1980; Yamada and Nishijima 1980; and Li et al. 1982), semicircular with a small pitch (Mehta and Raja Rao 1979; Gupta and Raja Rao 1979), and semicircular with a large pitch (Ganeshan and Raja Rao 1982).

Hijikata et al. (1987) and Hijikata and Mori (1987) measured local heat-transfer coefficients for different transverse disruptions in rectangular channels with air and in tubes with a water solution. Figure 2.7 shows the local heat-transfer coefficient $\left(\mathrm{Nu}_{\mathrm{x}}\right)$ variations for three different shapes - square, semicircular (sine), and arc - with the same disruption height and. 

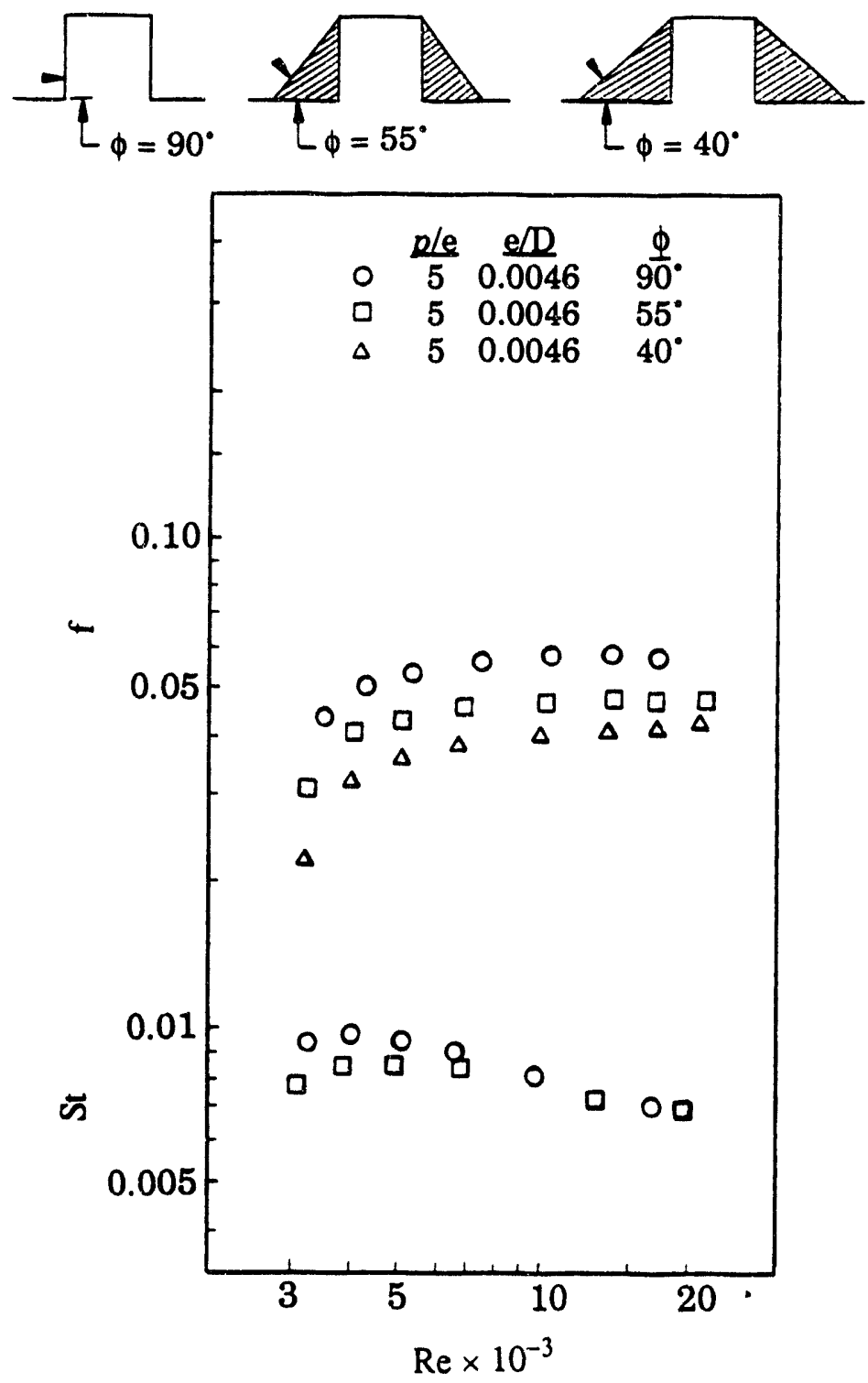

FIGURE 2.5 Friction-Factor and Heat-Transfer Performance Variations with Different

Disruption Shapes (Source: Han et al. 1978) 


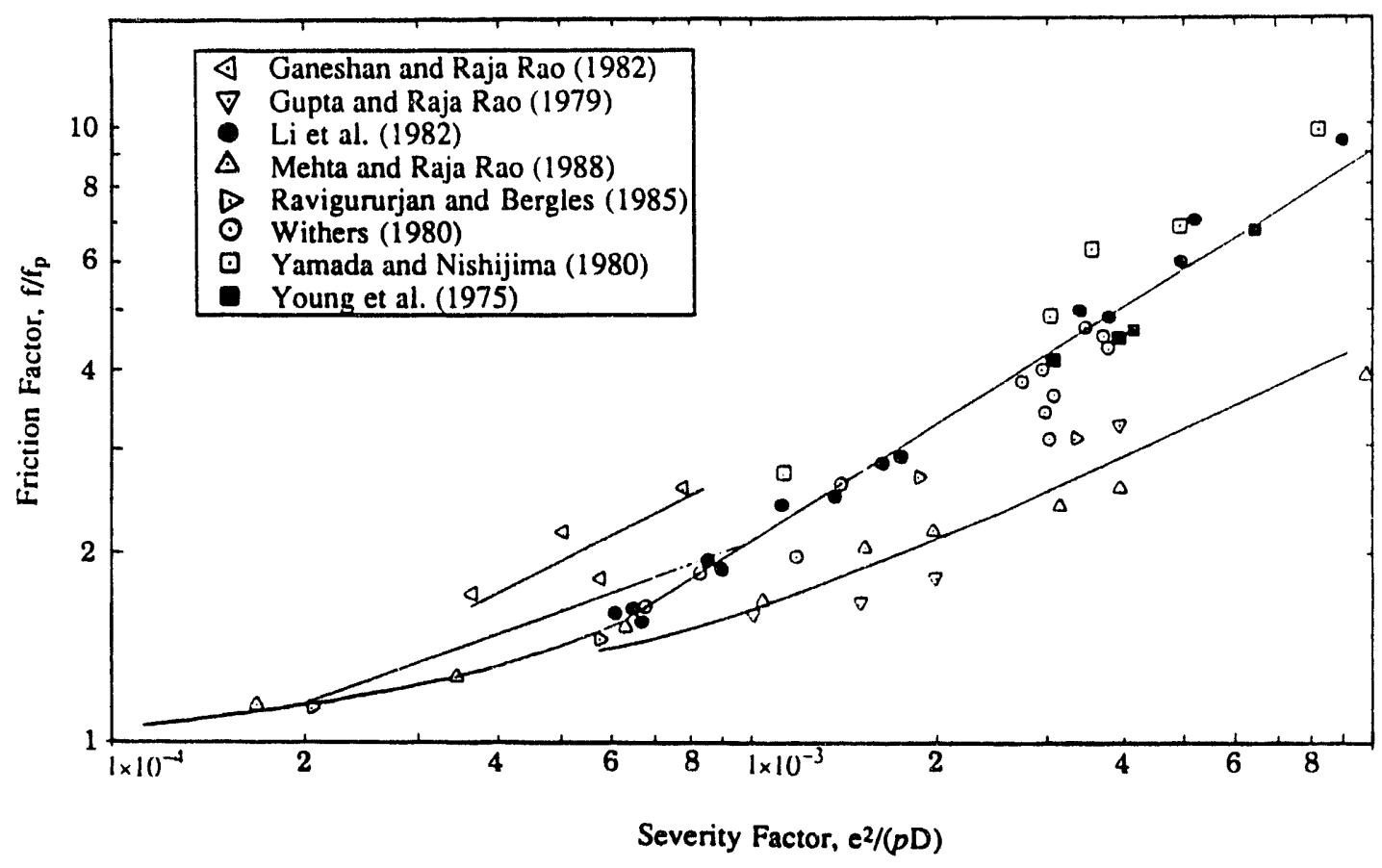

FIGURE 2.6 Friction-Factor Enhancement-Ratio Variation for Spirally Indented Tubes (Source: Rabas et al. 1988)

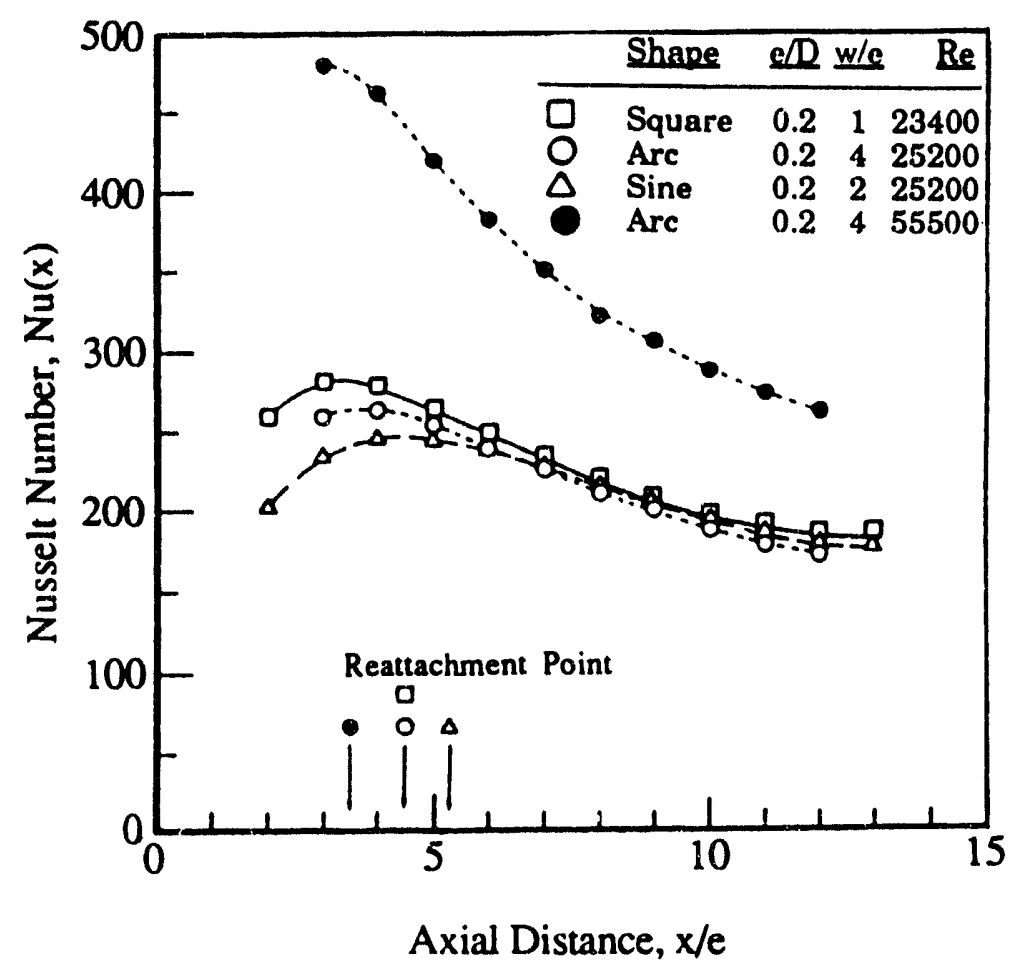

FIGURE 2.7 Local Heat-Transfer Coefficient Variation with Different Disruption Shapes in a Rectangular Passage (Source: Hijikata ei al. 1987) 
pitch. The rectangular passage shape was used. Note that the thermal performance is a function of the shape in the recirculation region upstream of the reattachment point, but that it is essentially independent of the shape downstream of the attachment point. This finding shows that the shape effect on the thermal performance is more significant when the disruption pitch is close to the reattachment location, but that it is less important for longer pitches. Figure 2.8 is a presentation similar to Figure 2.7; however, the local heat-transfer coefficients obtained with two arc (sine) shapes are compared with that of a wing or asymmetric shape. These disruptions were placed inside a circular passage. Note that the thermal performance of the wing-shaped disruption is substantially less than that obtained with the arc-shaped disruption for the same height and Reynolds number. Of course, the pressure drop is less - about $23 \%$ - with the wing-shaped disruption. This substantial change in the thermal performance may also be the result of some Prandtl number (Pr) effect (only comparison not using air), as well as the change to an asymmetric shape. Figures 2.7 and 2.8 also show that the attachment point depends on the disruption shape; in general, the reattachment length increases with an increase of curvature.

Sufficient experimental data exist - both mean and local - to demonstrate that the disruption shape is a significant parameter that affects the pressure-drop and, to a smaller extent, the heat-transfer performance of enhanced tubes with the separation and reattachment mechanism. However, a term to account for the effect of the shape is incorporated into only one prediction method that is recommended for the separation and attachment mechanism - that of Ravigururajan

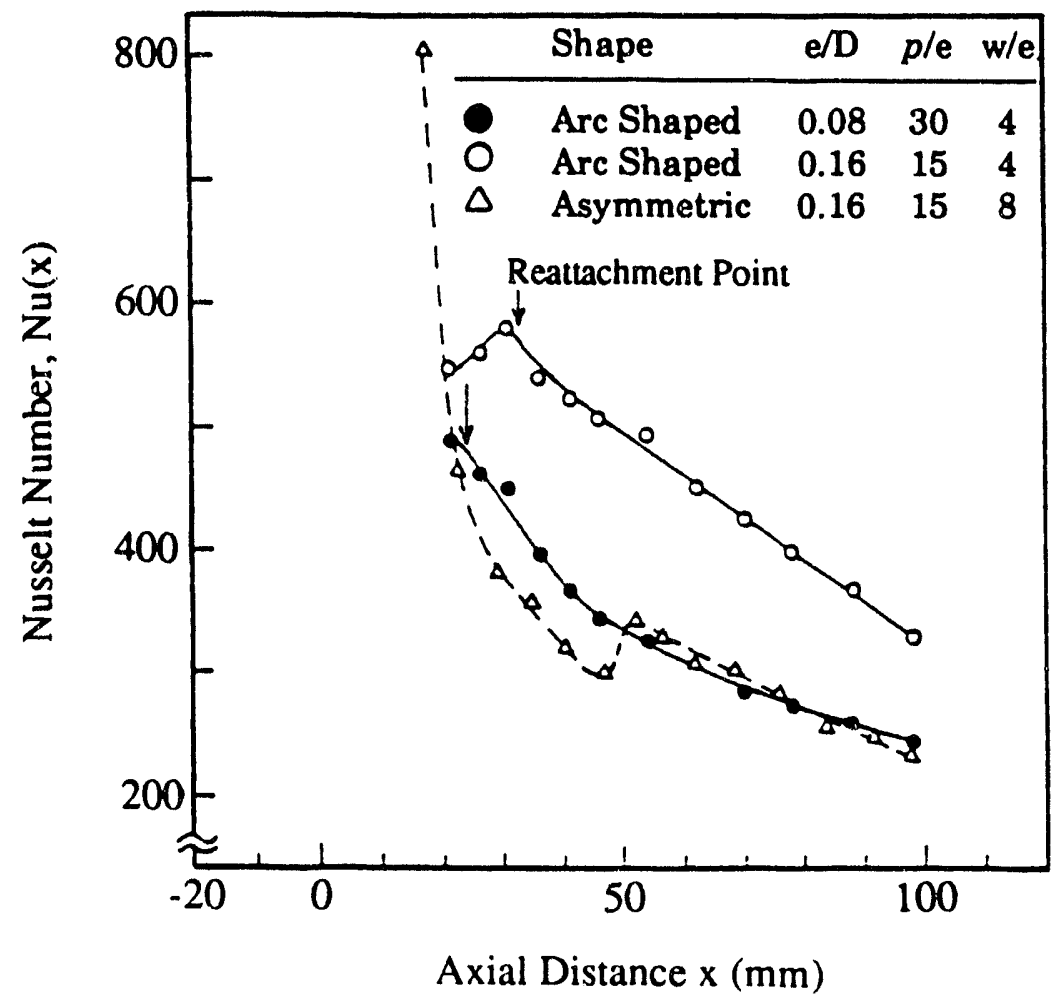

FIGURE 2.8 Local Heat-Transfer Coefficient Variation with Different Disruption Shapes inside an Enhanced Tube at $R e=20,000$ (Source: Hijikata et al. 1987) 
and Bergles $(1985,1986 \mathrm{a}, 1986 \mathrm{~b})$. This correction term is only for the friction-factor prediction; however, this correlation term contains only a few shape options and does not have the flexibility to evaluate the performance of untested shapes.

\subsection{Influence of the Prandtl Number}

Perhaps one of the least defined aspects of enhanced surfaces is the influence of the Prandtl number. Existing data clearly suggest that a simple power relation, such as $\mathrm{Nu} \propto \operatorname{Pr}^{\mathrm{m}}$, does not apply, especially when $\operatorname{Pr}$ is greater than unity. A number of experimental investigations have obtained heat-transfer data for a range of Prandtl numbers but only for enhancement mechanisms other than the separation and reattachment type. Dipprey and Sabersky (1963) and Gowen and Smith (1968) used three-dimensional, internally roughened passages (the boundary-layer thinning mechanism) to obtain heat-transfer data with water at different temperature levels. Dawson and Trass (1972) also used three-dimensional roughness surfaces to obtain mass-transfer data with Schmidt numbers that varied from 1,000 to 7,000. Smith and Gowen (1965) used an internally threaded pipe (the separation and recirculation mechanism) to compare the thermal performance obtained with water and Ucon (a polyalkylene glycol, $\operatorname{Pr}=349$ ). Carnavos $(1979,1980)$ used internally finned tubes (the extended-surface enhancement mechanism) to obtain experimental heattransfe $i$ data for both air and water. Further reference to these publications will not be made because they present data for mechanisms other than the separation and reattachment type.

Burck (1970), being aware of the importance of the Prandtl number, presented a very comprehensive evaluation of the data existing at that time, but he did not make a distinction between the various enhancement mechanisms. Burck concluded that the influence of the Prandtl number could be displayed with the efficiency index, $\eta=\left(S t / S t_{p}\right) /\left(f / f_{p}\right)$ (where $S t_{p}$ is the mean or spatial average Stanton number for a plain tube, and $f_{p}$ is the friction factor for a plain tube), as a function of the equivalent sand-grain roughness Reynolds number, $\mathrm{e}_{\mathrm{gg}}^{+}$, as shown in Figure 2.9. Note that the efficiency index increases with the Prandtl number, and values greater than unity can be obtained only with the higher Pr fluids.

Burck stated that this curve could be used to calculate the heat transfer for all types of enhanced surfaces if the friction factor and the Prandtl number are known. The majority of the data used to develop this figure were obtained with closed-spaced triangular grooves (Burck's data) and the data of Dipprey and Sabersky (1963). Table 2.1 shows that there are substantial errors between this prediction method and the data of Webb et al. (1971) (Section 2.3.3). Appendix A presents the calculation procedure used to obtain the predicted results.

Five publications have presented heat-transfer or mass-transfer data for tubes with enhancement geometry that is characteristic of the separation and reattachment mechanism. The authors of these publications include Savage and Myers (1963), Kalinin et al. (1970), Webb et al. (1971), Berger and Hau (1979), and Tan and Xaio (1989). This section presents a brief summary of each of these publications. 


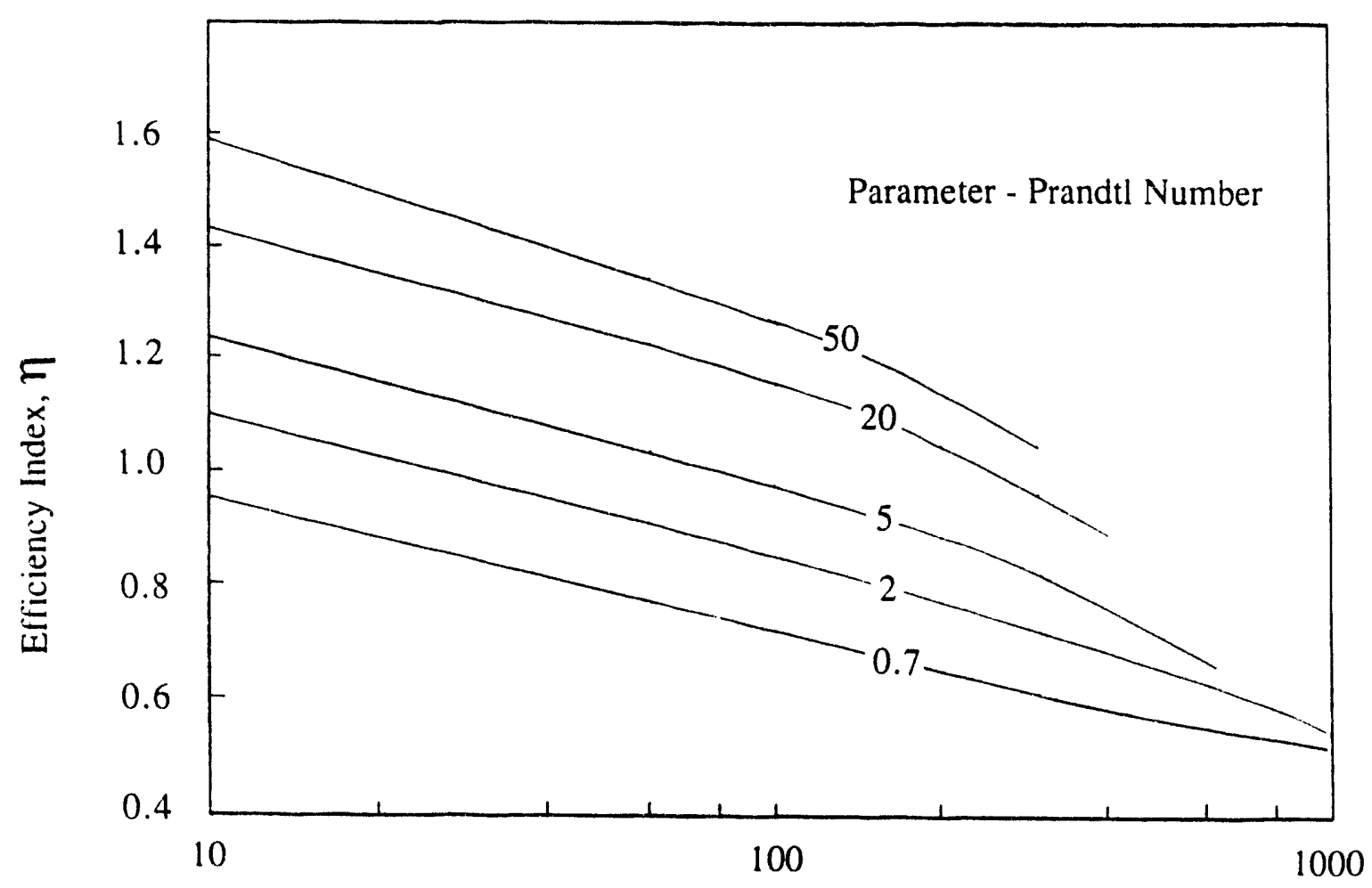

Equivalent Sand-Grain Roughness Reynolds Number, $\mathrm{e}_{\mathrm{sg}}^{+}$

FIGURE 2.9 Efficiency-Index Variation with the Equivalent Sand-Grain Roughness Reynolds Number (Source: Burck 1970)

\subsubsection{Savage and Myers (1963)}

Savage and Myers (1963) conducted heat-transfer and pressure-drop experiments with rectangular disruptions in a circular pipe with water as the test fluid. The water temperature was varied to investigate the Prandtl number effect for a limited range $(2<\operatorname{Pr}<8)$. The heat-transfer approached the heated disruption with a uniform, rather than a nonuniform, temperature distribution; the latter, of course, would exist if the upstream disruptions were thermally active. Because of the local heating, the authors stated that their heat-transfer data were of limited value for predicting the thermal performance when heating occurs for the entire tube length.

\subsubsection{Kalinin ot al. (1970)}

An extensive analysis of the heat-transfer and pressure-drop performance of transverse, grooved tubes was conducted in the USSR in the 1960s and summarized by Kalinin et al. (1970). Most of the data were taken with air as the test fluid, but a limited number of water data appear to have been taken but were not presented in the paper. Correlations were presented in the form. 
TABLE 2.1 Comparison of the Experimental (Webb et al. 1971) and Predicted (Burck 1970) Efficiency-Index Values ${ }^{a}$

\begin{tabular}{|c|c|c|c|c|c|c|}
\hline $\operatorname{Pr}$ & $R_{\theta}$ & $f$ & $\theta_{s g} / D$ & $\theta_{s g}^{+}$ & $\eta_{\text {Burck }}$ & $\eta_{\text {Exp }}$ \\
\hline \multicolumn{7}{|c|}{ Tube $01 / 10$} \\
\hline 0.71 & 6000 & 0.0130 & 0.04282 & 20.71 & 0.882 & 0.638 \\
\hline 0.71 & 10000 & 0.0161 & 0.07938 & 71.22 & 0.754 & 0.585 \\
\hline 0.71 & 40000 & 0.0202 & 0.12912 & 519.07 & 0.565 & 0.524 \\
\hline 5.10 & 6000 & 0.0130 & 0.04282 & 20.71 & 1.159 & 0.811 \\
\hline 5.10 & 10000 & 0.0161 & 0.07938 & 71.22 & 1.017 & 0.831 \\
\hline 5.10 & 40000 & 0.0202 & 0.12912 & 519.07 & 0.706 & 0.625 \\
\hline 21.70 & 6000 & 0.0130 & 0.04282 & 20.71 & 1.362 & 1.018 \\
\hline 21.70 & 10000 & 0.0161 & 0.07938 & 71.22 & 1.210 & 1.109 \\
\hline 21.70 & 40000 & 0.0202 & 0.12912 & 519.07 & 0.809 & 0.747 \\
\hline 37.60 & 6000 & 0.0130 & 0.04282 & 20.71 & 1.439 & 1.000 \\
\hline 37.60 & 10000 & 0.0161 & 0.07938 & 71.22 & 1.283 & 1.223 \\
\hline 37.60 & 40000 & 0.0202 & 0.12912 & 519.07 & 0.848 & 0.724 \\
\hline
\end{tabular}

Tube $02 / 10$

$\begin{array}{rrrrrrr}0.71 & 6000 & 0.0250 & 0.19447 & 130.46 & 0.693 & 0.520 \\ 0.71 & 10000 & 0.0302 & 0.27003 & 331.82 & 0.604 & 0.471 \\ 0.71 & 40000 & 0.0354 & 0.34775 & 1850.60 & 0.495 & 0.364 \\ \mathbf{5 . 1 0} & 6000 & 0.0250 & 0.19447 & 130.46 & 0.939 & 0.893 \\ 5.10 & 10000 & 0.0302 & 0.27003 & 331.82 & 0.795 & 0.687 \\ 21.70 & 6000 & 0.0250 & 0.19447 & 130.46 & 1.121 & 1.065 \\ 21.70 & 10000 & 0.0302 & 0.27003 & 331.82 & 0.936 & 0.787 \\ 37.60 & 6000 & 0.0250 & 0.19447 & 130.46 & 1.190 & 1.128 \\ 37.60 & 1000 & 0.0302 & 0.27003 & 331.82 & 0.990 & 0.828\end{array}$

Tube $02 / 20$

$\begin{array}{rrrrrrr}0.71 & 6000 & 0.0186 & 0.10887 & 62.99 & 0.766 & 0.609 \\ 0.71 & 10000 & 0.0202 & 0.12912 & 129.77 & 0.693 & 0.597 \\ 0.71 & 40000 & 0.0259 & 0.20729 & 943.56 & 0.523 & 0.423 \\ 5.10 & 6000 & 0.0186 & 0.10887 & 62.99 & 1.032 & 1.016 \\ 5.10 & 10000 & 0.0202 & 0.12912 & 129.77 & 0.940 & 0.868 \\ 5.10 & 40000 & 0.0259 & 0.20729 & 943.56 & 0.547 & 0.475 \\ 21.70 & 6000 & 0.0186 & 0.10887 & 62.99 & 1.226 & 1.215 \\ 21.70 & 10000 & 0.0202 & 0.12912 & 129.77 & 1.122 & 0.991\end{array}$


TABLE 2.1 (Cont'd)

\begin{tabular}{|c|c|c|c|c|c|c|}
\hline $\operatorname{Pr}$ & $R e$ & $f$ & $\theta_{39} / D$ & $\theta_{s g}^{+}$ & $\eta_{\text {Burck }}$ & $\eta_{\operatorname{Exp}}$ \\
\hline \multicolumn{7}{|c|}{ Tube $02 / 40$} \\
\hline 0.71 & 6000 & 0.0141 & 0.04653 & 23.44 & 0.869 & 0.605 \\
\hline 0.71 & 10000 & 0.0151 & 0.04451 & 38.67 & 0.817 & 0.648 \\
\hline 0.71 & 40000 & 0.0165 & 0.08390 & 304.82 & 0.611 & 0.524 \\
\hline 0.71 & 100000 & 0.0172 & 0.09200 & 853.13 & 0.529 & 0.422 \\
\hline 5.10 & 6000 & 0.0141 & 0.04653 & 23.44 & 1.145 & 1.019 \\
\hline 5.10 & 10000 & 0.0151 & 0.04451 & 38.67 & 1.089 & 0.928 \\
\hline 5.10 & 40000 & 0.0165 & 0.08390 & 304.82 & 0.810 & 0.615 \\
\hline 21.70 & 6000 & 0.0141 & 0.04653 & 23.44 & 1.348 & 1.188 \\
\hline 21.70 & 10000 & 0.0151 & 0.04451 & 38.67 & 1.288 & 1.042 \\
\hline 21.70 & 40000 & 0.0165 & 0.08390 & 304.82 & 0.957 & 0.660 \\
\hline 37.60 & 6000 & 0.0141 & 0.04653 & 23.44 & 1.425 & 1.290 \\
\hline 37.60 & 10000 & 0.0151 & 0.04451 & 38.6 .7 & 1.364 & 1.111 \\
\hline 37.60 & 40000 & 0.0165 & 0.08390 & 304.82 & 1.012 & 0.654 \\
\hline \multicolumn{7}{|c|}{ Tube $04 / 10$} \\
\hline 0.71 & 6000 & 0.0592 & 0.69584 & 718.30 & 0.541 & 0.306 \\
\hline 0.71 & 10000 & 0.0597 & 0.70278 & 1214.20 & 0.509 & 0.286 \\
\hline 5.10 & 6000 & 0.0592 & 0.69584 & 718.30 & 0.626 & 0.465 \\
\hline 5.10 & 10000 & 0.0597 & 0.70278 & 1214.20 & 0.459 & 0.400 \\
\hline 21.70 & 6000 & 0.0592 & 0.69584 & 718.30 & 0.689 & 0.521 \\
\hline
\end{tabular}

a See nomenclature for definition of terms.

$\mathrm{Nu} / \mathrm{Nup}=\mathrm{f}(\mathrm{Re}, \mathrm{e} / \mathrm{D}, p / \mathrm{e})$ with no Prandtl number correction. It will be shown that this finding is not in agreement with the other data presented in this report. A possible explanation for this difference could be that only limited water data were available for this correlation effort.

\subsubsection{Webb et al. (1971)}

Webb et al. (1971) presented data obtained for a broad Prandtl number range with an enhanced tube geometry characteristic of the separation and reattachment mechanism. They used four different fluids in their tenchmark experiments, the Prandtl numbers of which are as follows: air $=0.71$, water $=5.1$, n-butyl alcohol $=21.7$, and butyl alcohol $=37.6$. Five different tube geometries with transverse, rectangular disruptions or ribs were tested (Table 2.2). 
Figure 2.10 shows the measured heat-transfer enhancement values plotted as a function of the roughness Reynolds number with the Prandtl number as a parameter for the $01 / 10$ tube tested by Webb et al. (1971). The maximum enhancement level is very pronounced, and the level increases as the Prandtl number increases. The maximum enhancement level occurs at a lower $\mathrm{e}^{+}$and the $\mathrm{e}^{+}$range decreases as the Prandtl number increases. Curves of the data for the other tubes are very similar in shape, with the major difference being the locations and the magnitude of the enhancement levels. This characteristic of the enhancement level suggests a geometry-selection procedure based on the roughness Reynolds number as described by Webb (1979). However, there is no satisfactory explanation as to why the Prandtl number affects the enhancement level in the manner shown in Figure 2.10.
TABLE 2.2 Tests of Tube Geometries ${ }^{a}$

\begin{tabular}{|c|c|c|c|}
\hline $\begin{array}{l}\text { Tube } \\
\text { Number }\end{array}$ & $\Theta / D^{b}$ & $p / \theta$ & $w / \theta$ \\
\hline $01 / 10$ & 0.01 & 10 & 1.034 \\
\hline $02 / 10$ & 0.02 & 10 & 0.517 \\
\hline $04 / 10$ & 0.04 & 10 & 0.259 \\
\hline $02 / 20$ & 0.02 & 20 & 0.517 \\
\hline $02 / 40$ & 0.02 & 40 & 0.517 \\
\hline \multicolumn{4}{|c|}{$\begin{array}{l}\theta=\text { disruption height; } \\
p=\text { disruption pitch; and } \\
w=\text { disruption width. }\end{array}$} \\
\hline \multicolumn{4}{|c|}{$\mathrm{b} D=36.8 \mathrm{~mm}$ (1.45 in.). } \\
\hline
\end{tabular}

Figures 2.11 and 2.12 present the Reynolds number and Prandtl number exponents based on the Webb et al. (1971) data for tubes $04 / 10$ and $01 / 10$, respectively. These exponents were obtained from second-order polynomial curve fits of the experimental data in the form $\ln (\mathrm{Nu})$ with $\ln (\mathrm{Re})$ for fixed $\mathrm{Pr}$ and $\ln (\mathrm{Nu})$ with $\ln (\mathrm{Pr})$ for fixed Re. Also shown in these figures are the Reynolds number exponent, $n$, and the Prandtl number exponent, $m$, values for a plain tube based on the commonly used Petukhov and Popov correlation (Petukhov 1970). Both figures show that the variations of the exponents for the two enhanced tubes are very different from a plain tube and also differ from each other. For the smooth tubes, both exponents increase with the Reynolds number for a fixed Prandtl number, whereas almost the opposite is obtained for the enhanced tubes, with the only exception being the Prandtl number exponent at Pr greater than about 10. In general, the Prandtl number exponents for the two enhanced tubes and the smooth tube are not too different; they essentially decrease with an increase of the Prandtl number and are bounded by about 0.65 for $\operatorname{Pr}=0.7$ to about 0.35 for very large $\operatorname{Pr}$ values.

The most dramatic variation occurs with the Reynolds number exponents, especially for the 01/10 tube geometry. Note that the exponent changes from increasing to decreasing with a fixed Pr as the Reynolds number increases. For the lower Re values, there is a significant thermalperformance improvement with increasing Prandtl number; however, this improvement occurs with the $01 / 10$ tube at low $\operatorname{Re}$ values but not for the $04 / 10$ tube. For the $04 / 10$ tube, there is a general decrease of $\mathrm{n}$ with $\mathrm{Pr}$, and it appears to reach different asymptotic values that are a function of the $\operatorname{Re}$ value. For the larger $\operatorname{Re}$ values, $n$ is in the 0.65 to 0.72 range. The magnitude of $n$ for the larger disruption or rib and the largest Reynolds number range is an important finding that will 


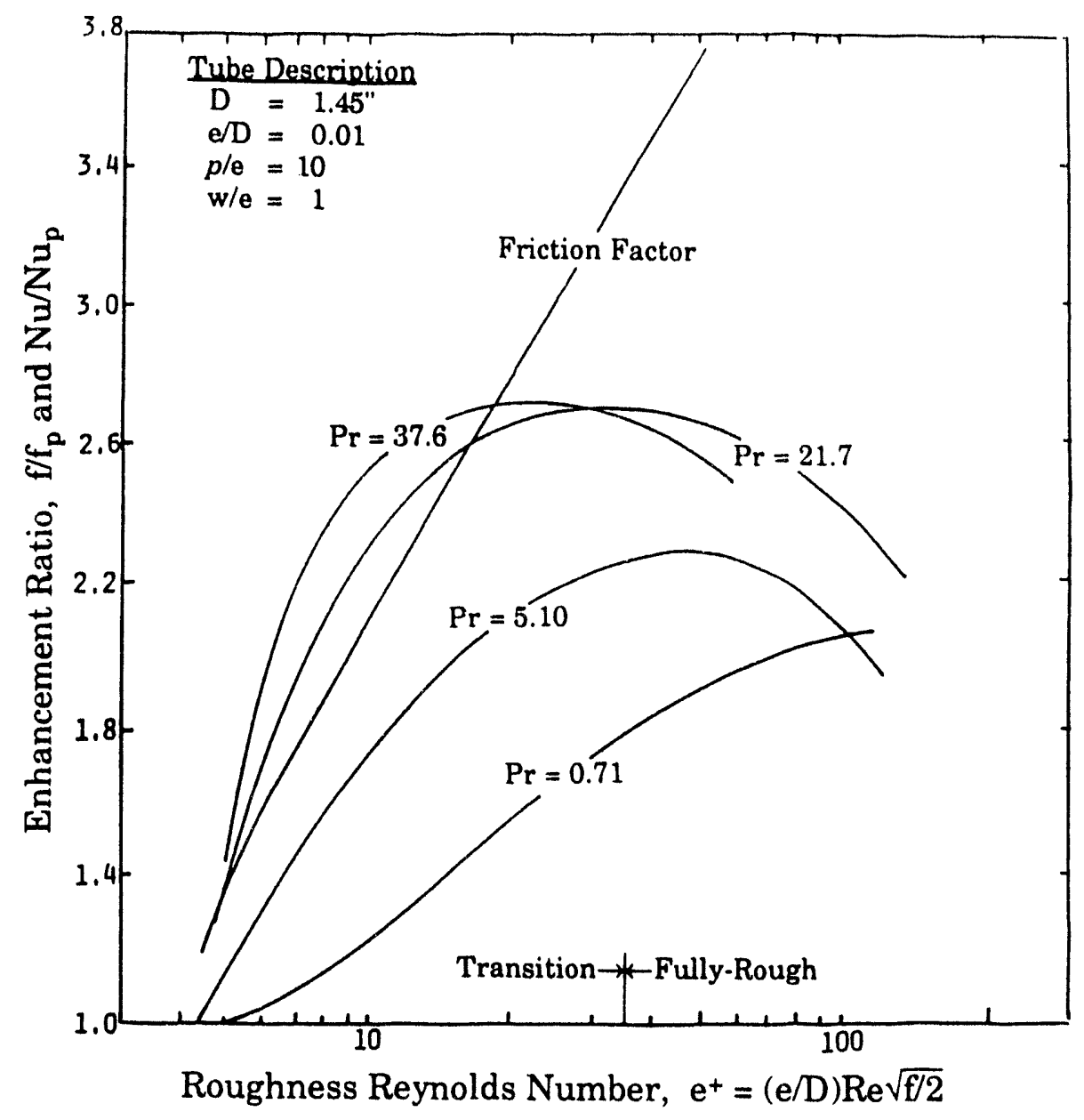

FIGURE 2.10 Heat-Transfer Enhancement as a Function of the Roughness Reynolds Number (based on data for the 01/10 tube geometry) (Source: Webb et al. 1971)

be discussed later. In addition, the sometimes used Wilson plot method for data reduction can be questioned because the heat-transfer coefficient is assumed to have a fixed-exponent dependence on the velocity or Reynolds number.

\subsubsection{Berger and Hau (1979)}

Berger and Hau (1979) used the electrochemical analogue technique to measure the local and mean mass-transfer performance in a pipe with transverse, square disruptions with a heignt-todiameter ratio of 0.0364 and with different pitch-to-height ratios. The Schmidt number varied over a wide range - from 1,000 to 7,000 . The major findings for the $036 / 10$ tube were as follows: 


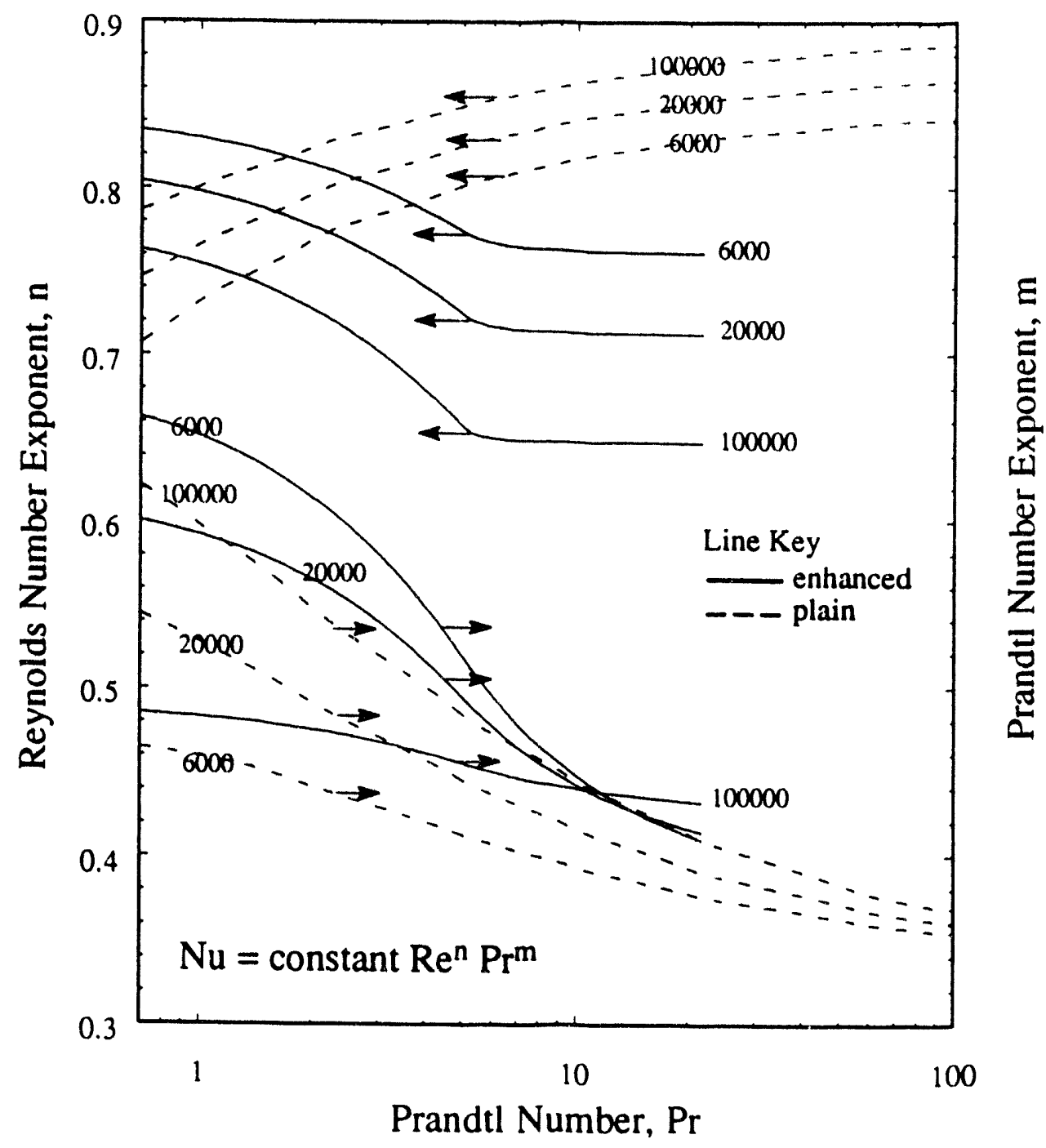

FIGURE 2.11 Reynolds Number and Prandtl Number Exponents as a Function of the Prandtl Number for the 04/10 Tube Geometry (Source: Webb et al. 1971)

1. The Prandtl number exponent did not vary from a value of about 0.33 .

2. The exponent on the Reynolds number was 0.695 - substantially lower than that obtained with smooth-tube prediction methods (about 0.85 ).

3. The variations between the maximum and minimum local mass-transfer values and the mean value decreased with increasing Reynolds number; in other words, the mass-transfer distribution became more uniform. The maximum values were located at the top of the rib and near the reattachment point, and the minimum values were located directly in front of and behind the rib. 


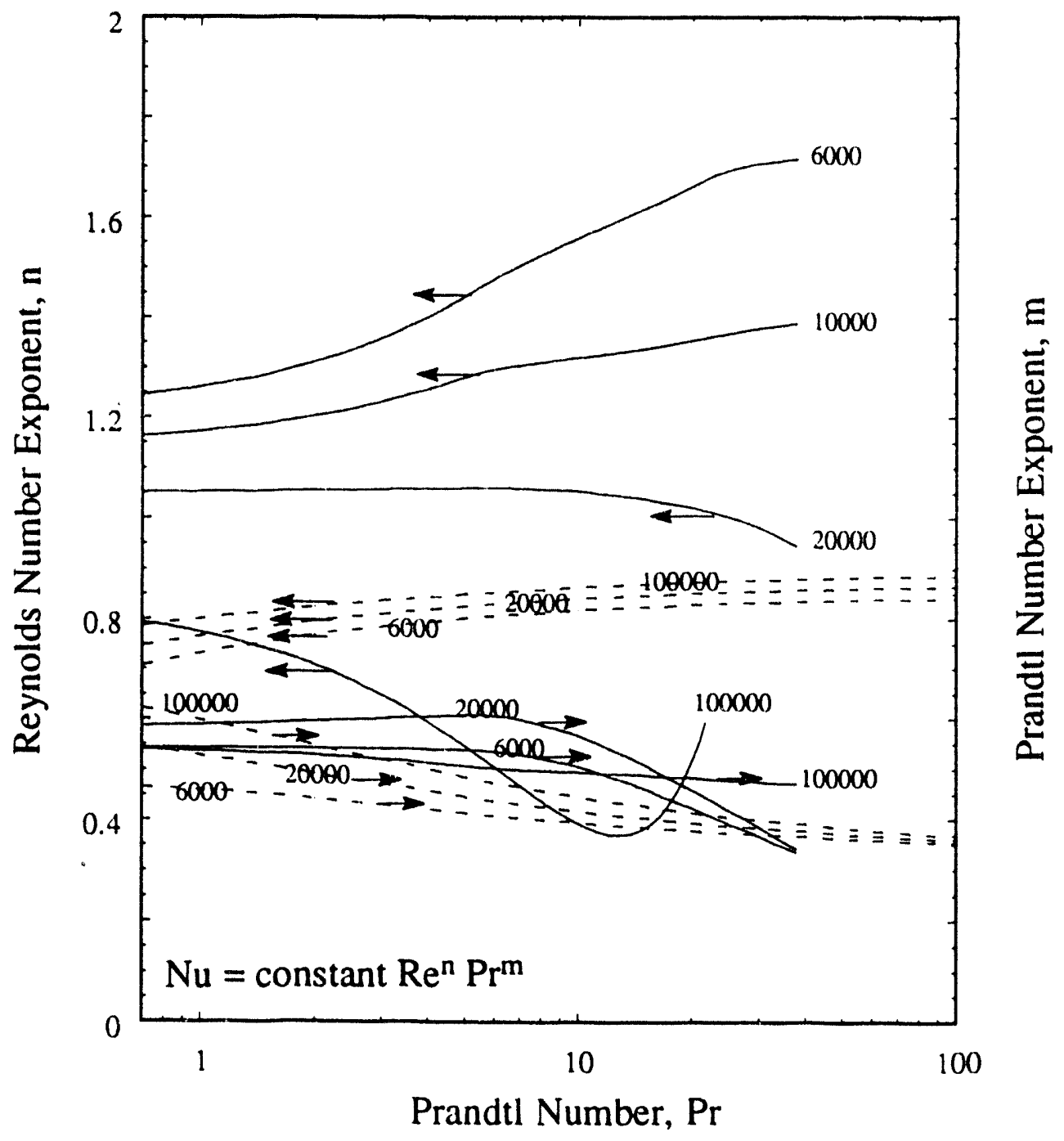

FIGURE 2.12 Reynolds Number and PrandtI Number Exponents as a Function of the Prandtl Number for the 01/10 Tube Geometry (Source: Webb et al. 1971) 
4. The point of maximum heat transfer moved closer to the rib as the Reynolds number increased.

Note that the first two observations for the $036 / 10$ tube are consistent with the data of Webb et al. (1971) for the 04/10 tube shown in Figure 2.11. The local data, which were used to obtain the last two observations, offer a fundamental understanding of how the Prandtl number influences the performance; unfortunately, these data were obtained with large Schmidt number values.

\subsubsection{Tan and Xaio (1989)}

Figure 2.13, based on the data of Tan and Xaio (1989), also shows the enhancement levels as a function of the roughness Reynolds number with the Prandtl number as a parameter. These results are for a tube with a contoured disruption shape. The tube is the single-start, spirally indented type with $e / \mathrm{D}_{\mathrm{i}}$ and $p / \mathrm{e}$ values of approximately 0.053 and 10.8 , respectively. The indenting process produces internal disruptions that are rounded or contoured without any sharp corners. Note the similarity between these data and the data obtained by Webb et al. (1971), shown in Figure 2.10. For air $(\operatorname{Pr} \approx 0.7)$, the enhancement level increases with $\mathrm{e}^{+}$until a maximum is obtained. For liquids ( $\operatorname{Pr}>1$ ), a maximum enhancement level is obtained; however, the maximum occurs at smaller $\mathrm{e}^{+}$values $\left(\mathrm{e}^{+} \approx 20\right)$ and is less Prandtl-number-dependent than the maximum obtained for rectangular disruptions or ribs.

The experimental data shown in Figures 2.10 and 2.13 clearly demonstrate that the Prandtl number has an important impact on the performance of enhanced tubes with geometries characteristic of the separation and reattachment mechanism. The recommended correlation of Kalinin et al. (1970) that does not include the Prandtl number certainly cannot be correct. The purpose of this report is to explain this Prandtl number effect using fluid-mechanics and heattransfer results obtained with a numerical modeling approach. A brief description of the numerical model and its validation is presented in the following sections of this report. 


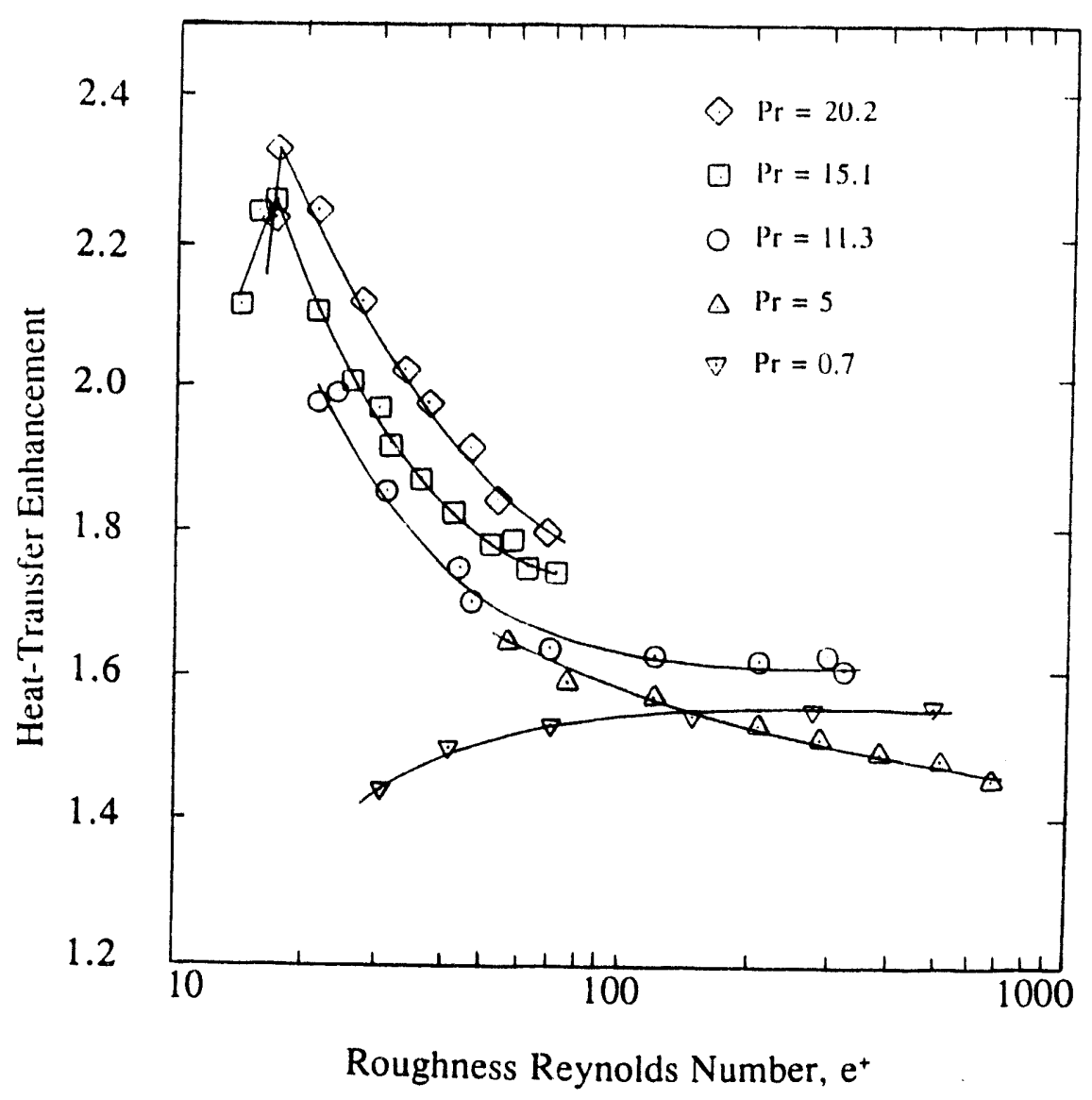

FIGURE 2.13 Heat-Transfer Enhancement as a Function of the Roughness Reynolds Number (Source: Tan and Xaio 1989) 


\section{Previous Numerical Analysis Investigations}

The discussion in Sections 1 and 2 demonstrated the need for research into the shape and Prandtl number effects on the thermal-hydraulic performance for the separation and reattachment mechanism. The method selected for this research is numerical analysis. The major reasons for this selection are as follows: (1) the recent progress made in numerical modeling, (2) the improvement of computer hardware capability, and (3) the relatively simple required analysis that is, a two-dimensional rather than a three-dimensional analysis is required. It was felt that other methods, such as classical analysis and recorrelation of the data, would not reveal the fluidmechanic and heat-transfer details as well as the numerical ricthod.

This section presents some background on the recent progress in numerical analysis, discusses the heat-transfer modeling studies for some benchmark internal flows (backward-facing steps, internal contractions, and expansions), and reviews the eight previous numerical-analysis investigations devoted to heat-transfer predictions in enhanced passages. Both turbulent and laminar flow will be discussed, although the focus of this report centers on the more important turbulent-flow case. For completeness, Appendix B contains brief descriptions of commonly used concepts and models, including the algebraic-viscosity model, the $k-\varepsilon$ model, and large-scale eddy simulation.

\subsection{Background}

The purpose of this background discussion is to alter the conventional thinking that the thermal performance improvement obtained with enhanced tubes can be explained in terms of the Reynolds-analogy mechanism. Launder (1988) presented an excellent review on the computation of convective heat transfer in turbulent flows. He considered every flow field as "simple" if the velocity and temperature profiles close to the wall conformed to the so-called universal logarithmic laws, written as:

$$
\begin{aligned}
& u^{+}=1 / k \ln \left(E y^{+}\right) \\
& T^{+}=1 / k^{\prime} \ln \left(E^{\prime} y^{+}\right)
\end{aligned}
$$

where:

$$
\begin{aligned}
\mathrm{u}^{+} & =\text {axial velocity (dimensionless); } \\
\mathrm{K} & =\text { von Karman constant, } \sim 0.42 \\
\mathrm{E} & =\text { empirical constant, } \sim 9.8
\end{aligned}
$$




$$
\begin{aligned}
& \mathrm{y}^{+}=\text {distance from wall (dimensionless); } \\
& \mathrm{T}^{+}=\text {temperature (dimensionless); } \\
& \mathbf{K}^{\prime}=\text { empirical constant, } \sim 0.46 ; \text { and } \\
& \mathrm{E}^{\prime}=\text { empirical constant (a function only of the Prandtl number). }
\end{aligned}
$$

Only boundary-layer flows, where the Reynolds-analogy concept applies, are in the "simple" category; all of the others, including separating and reattaching flows, are in the complex category.

Launder stated that the logarithmic temperature law is less widely valid than the velocity law. The logarithmic temperature law ceases to be applicable for the following conditions: (1) when the near-wall turbulence departs from a state of local equilibrium, (2) when the thermal boundary condition varies rapidly in the flow direction, or (3) when the thermal layer is very thin. All of these conditions are present with geometries that are characteristic of the separation and reattachment enhancement mechanism.

Launder (1988) described two different numerical modeling strategies adopted for complex flows (i.e., when the logarithmic laws no not apply): (1) refine the boundary-layer or analogy equations while retaining the broad philosophy that underlies them or (2) discard them in favor of detailed modeling of the turbulent transport across the near-wall sublayer. The early efforts employed the first strategy for complex flows and obtained very unsatisfactory results. Launder explicitly stated that the first approach could not be used for separated flows because, if the Reynolds-analogy concept applies, the local heat-transfer coefficient would fall to zero at the stagnation or reattachment points; however, experiments have shown that the maximum heattransfer coefficients occur at or near the reattachment points. The transport of highly turbulent fluid with large-scale fluctuations to the surface is the primary mechanism for the augmentation of the heat transfer in the vicinity of a stagnation point. The two major stagnation points with the separation and reattachment geometry occur at the upstream face of the disruption and at the reattachment point, as shown in Figure 1.1.

The so-called mechanistic correlation approach used by the heat-transfer community for the separation and reattachment enhancement mechanism is based on the above equations or on the assumption that the flow field falls into the simple-flow category. Cases in point are the correlations of Webb et al. (1971), Withers (1980), Li et al. (1982), Ganeshan and Raja Rao (1982), Nakayama et al. (1983), Sethumadhavan and Raja Rao (1983), and Mehta and Raja Rao (1988). It will be shown in the following sections that employing this approach does not capture the actual enhancement mechanism that exists with separating and reattaching (stagnation) flow fields. 


\subsection{Backward-Facing Stops}

A vast number of numerical works exist for backward-facing steps because this geometry is often used to validate a new turbulence model or numerical scheme. Despite this abundant information, the challenge of properly modeling backward-facing steps still remains. This report will review selected studies that involve both the heat-transfer and fluid-dynamic performance.

Gooray et al. (1985) studied the momentum- and heat-transfer performance in both a backward-facing step and a sudden pipe expansion by using both standard and low-Reynolds number $k-\varepsilon$ (where $k$ is turbulent kinetic energy, and $\varepsilon$ is turbulent energy dissipation) twoequation models. The models were modified to account for the effects of streamline curvature and pressure-strain interactions, including wall damping. Gooray et al. (1985) applied the standard k$\varepsilon$ model to the separated flow region before the reattachment point and a low Reynolds number model immediately downstream of the reattachment. The agreement in heat-transfer predictions with the experimental data was found to be satisfactory. It was also reported that uncertainty exists if this method is applied to different geometries.

During the early 1980s, the engineering turbulence modelers suggested that the one-point, wall-function approach was not valid for the flows with separation and reattachment. A common and ad-hoc way to circumvent the problem was to modify the current one-point wall functions by two-layer or even three-layer wall functions (Chieng and Launder 1980; Johnson and Launder 1982; Amano et al. 1983). Ciofalo and Collins (1989) critically evaluated the validity of standard wall functions and suggested their own modified two-layer wall functions. They observed that the improvements to the riear-wall models were due to the variation of the viscous sublayer thickness and/or the slope of the velocity in the logarithmic region of the standard wall functions. The modifications of wall-function constants $\mathrm{E}$ and $\mathrm{K}$ would produce the improvements while retaining the well-known standard wall functions. Generally, good agreement with experiments was achieved. The 0.7 power dependency of the Nusselt number on the local Reynolds number was calculated, whereas experiments suggest 0.67 .

Recently, convective heat-transfer performance downstream of a backward-facing step was investigated by Scherer and Wittig (1989). The reattachment length predicted with the k- $\varepsilon$ model was underestimated by about $10 \%$ to $20 \%$. The main conclusion of Scherer and Wittig (1989) was that the two-layer wall-function approach of Chieng and Launder (1980) was superior to the standard wall functions.

\subsection{Sudden Pipe Expansions}

A number of numerical works have focused on sudden pipe expansions. The essentials of the fluid mechanics and heat transfer are similar to the backward-facing steps; therefore, the challenge of improved modeling still remains. This report will review some studies that also consider the heat-transfer aspect. 
Chieng and Launder (1980) suggested that detailed modeling of the near-wall region (improved wall functions) was necessary to achieve a better prediction of the flow field by means of the $k-\varepsilon$ model. They developed a two-layer wall-function model that yielded an overall improvement in the near-wall profiles of velocities and turbulent kinetic energy. Generally, close agreement was obtained between calculated and predicted Nusselt numbers. The maximum heattransfer value was predicted to be about one step height upstream of the reattachment point. Chieng and Launder (1980) also used a low-Reynolds number version of the $k-\varepsilon$ model and found that the heat-transfer coefficients near the reattachment point were seven times higher. In a subsequent study, the cause of the problem was identified as the excessive level of length scales (too large of a turbulent diffusive transport of the kinetic energy) (Yap 1987). Johnson and Launder (1982) corrected some coding errors in the previous study and further improved the twolayer wall function by allowing the sublayer thickness to vary as a function of local Reynolds number. These corrections resulted in better agreement between the experimental and the predicted local Nusselt number variations. The new study revealed that the proposed wall function was not as good as was previously expected.

Amano (1983) used the same two-layer wall-function model as Chieng and Launder (1980) in conjunction with the standard $k-\varepsilon$ model, except for the treatment of the generation and destruction terms in the $\varepsilon$-equation. Their treatment yielded an overall improvement in the magnitude of Nusselt number predictions and the location of the maximum heat-transfer coefficient. In follow-up work, Amano et al. (1983) further developed a three-layer wall function that would result in a better fit to the experimental profiles than the two-layer wall function. This three-layer wall function resulted in an overall improvement in heat-transfer performance at highReynolds-number flows over the previous study; however, the low-Reynolds-number turbulent flows were insensitive to this wall-function model. The predicted location of the maximum heattransfer performance coincided with that of the experiments.

Amano and Goel (1984) employed a simplified version of the Reynolds stress model to simulate the heat-transfer performance of a backward-facing step. The calculated Reynolds stresses were used only to evaluate the turbulent kinetic-energy production term, and the Reynolds stresses and fluxes in the momentum and energy equations were calculated by using the standard Boussinesq concept. This model again employed wall functions for the velocities, temperature, and the turbulent kinetic-energy equation. The heat-transfer and flow-fluid results were improved compared with their standard $k-\varepsilon$ two-equation model results.

\subsection{Enhanced Passages}

This section presents the current state-of-the-art of turbulent numerical modeling of the heat-transfer performance of internally enhanced passages. Substantially more numerical-analysis publications focus on only the turbulent fluid mechanics of enhanced passages. Also, the number of publications that considers both the heat transfer and fluid mechanics with laminar flow greatly exceeds those that consider turbulent flow. This section of the report focuses on the turbulent heattransfer results, with some mention of the benchmark turbulent fluid-mechanics studies. The body' 
of work restricted to laminar flow is not discussed because it is not rele vant to the more important turbulent-flow case.

An extensive literature review uncovered only eight publications that presented numerical predictions of the turbulent heat-transfer performance inside enhanced passages. The authors of these publications include Taylor et al. (1991), Becker and Rivir (1989), Fodemski and Collins (1988), Hung et al. (1987), Hijikata et al. (1987), Fujita et al. (1986), Barba (1984), and Patankar et al. (1979). This section presents a brief summary of each of these publications.

\subsubsection{Taylor et al. (1991)}

Taylor et al. (1991) performed a numerical simulation of the turbulent flow within a stationary and rotating cylindrical tube with multiple transverse disruptions. The tube diameter and length were 0.01 and $0.13 \mathrm{~m}$, respectively. Four axisymmetric, square ribs of $0.003 \mathrm{~m}$ in height and $0.002 \mathrm{~m}$ in width were placed at $0.02-\mathrm{m}$ intervals $(p / \mathrm{e}=6.67)$. The selected Reynolds number was 15,000 , and the test fluid was air. The geometry and flow conditions are pertinent to the gas-turbine blade-cooling applications.

This simulation used the full conjugate problem. In the finite-element analysis, a oneequation model was used in conjunction with a logarithmic universal law near the wall to depict the variations in the velocity component.

The numerical simulations were performed for both rotating and stationary passages. The major findings of this study were as follows:

1. There was a substantial increase in heat-transfer performance due to the disruption.

2. The Coriolis effects due to the rotation on the heat transfer were insignificant for the rotation rate and the Reynolds number range considered.

\subsubsection{Becker and Rivir (1989)}

Becker and Rivir (1989) determined the heat-transfer performance from a single disruption in a developing two-dimensional boundary-layer flow field. The selected, upstream velocity profile was as follows: outside of the boundary layer, a uniform velocity existed; inside of the boundary layer, the $1 / 7$ power law was assumed. The Reynolds number was 60,000 , and the fluid was air. The disruption height, e, was $1 \mathrm{~mm}$, and the shape was essentially rectangular ( $w=3 e$, where $w$ is the disruption width) with circular corners of radius $\sin (\pi x / 2 e)$, where $x$ is the axial coordinate. 
The turbulent closure was obtained with an algebraic-viscosity model, namely, the Baldwin-Lomax form of the Cebeci-Smith two-layer algebraic eddy-diffusivity model (Baldwin and Lomax 1978). The numerical algorithm used to calculate the results was the MacCormack explicit predictor-corrector technique (Anderson et al. 1984) in an orthogonal coordinate frame.

The predicted average Nusselt number was compared with experimental results obtained by Han et al. (1986) for a rectangular passage. The numerical predicted heat-transfer performance was greater than the experimental data by $34 \%$; however, the general character of the local Nusselt number variation after the disruption was consistent with the measurements. The major reasons for the disparity were the different rib geometry and size, the two-dimensional analysis (rather than the required three-dimensional analysis for a rectangular channel), the limitations of the turbulence model in the recirculation zones, and the different free-stream mean velocity distribution upstream of the disruption. A major conclusion of this investigation was that the Reynolds analogy greatly underpredicts the measured heat-transfer performance for this separation and reattachment flow mechanism. This conclusion is consistent with the previous background discussion of Launder (1988).

Discussions were held with Dr. Rivir concerning his current research dealing with turbulent modeling of enhanced passages. Dr. Rivir is completing an analysis of six disruptions by using essentially the same technique and hopes to supplement the numerical work with local heat-transfer coefficient data.

\subsubsection{Fodemskl and Collins (1988)}

Fodemski and Collins (1988) evaluated the fluid-flow and heat-transfer characteristics in a rectangular passage ( 4.0 aspect ratio) with one surface containing transverse square ribs with a $p / e=7.125$ and $e / D=0.11$. The bottom of the enhanced surface was heated (constant temperature), and the coupling between the convective heat transfer to air and conduction within this solid surface was also considered. This computer simulation of the temperature field was carried out over 10 ribs. A unique feature of this simulation was the consideration of the full conjugate problem.

The Harwell FLOW 3D (Computational Fluid Dynamics Services, Inc.) computer code was used. A standard $k-\varepsilon$ model and standard wall functions with a finite-volume method was employed with a fully developed velocity profile. To accommodate a fully developed flow field, wrap-around or cyclic boundary conditions were invoked, which implied identical inlet and outlet velocity profiles at the same location (e.g., the midpoint between the ribs). A detailed discussion of the wrap-around procedure is presented in Section 4.3. The overall iterative-solution procedure was based on the SIMPLE algorithm.

The predicted temperature field was in reasonable agreement with holographic interferometry measurements described in previous publications, except for the identical geometry. 
Figures 3.1 and 3.2, taken from Fodemski and Collins (1988), shows the Nusselt number variation for the first and the eighth ribs, respectively. Also shown with the results for the eighth rib are experimental interferogram data and additional predicted results of Wilkes (1981). Reasonable agreement exists, but substantial overprediction was obtained in the recirculation zone preceding the reattachment point.

\subsubsection{Hung et al. (1987)}

Hung et al. (1987) performed a two-dimensional turbulent-flow heat-transfer analysis with a single transverse rib inside a pipe. The effects of the rib height, rib width, and Reynolds number were determined for a fixed Prandtl number, $\operatorname{Pr}=0.7$. Uniform inlet velocity and temperature profiles were assumed, and a uniform tube-wall heat-flux boundary condition was selected. Very high rib heights were used, the variation being $\mathrm{e} / \mathrm{D}=0.125$ to 0.25 , which is higher than typical commercial-tube values of 0.01 to 0.06 . In addition, the rib-width range was outside the practical range; $w_{d} / e$ varied from 2 to 40 , rather than from 0.5 to 3 .

In the analysis, a $k-\varepsilon$ two-equation model was used to calculate the turbulent quantities, the centerline velocity and pressure, the wall shear stress, and the local Nusselt number. The

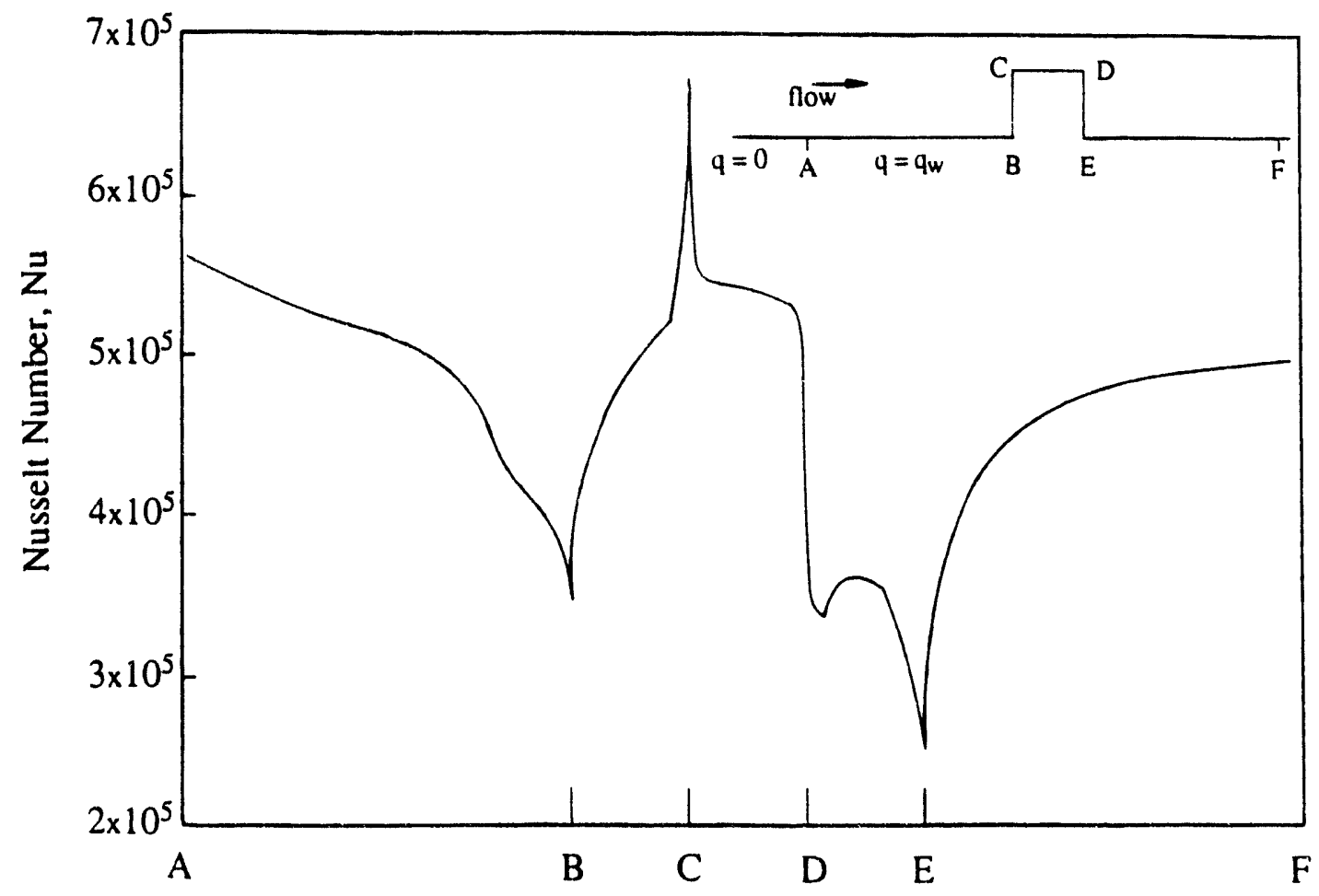

FIGURE 3.1 Nusselt Number for the First Heated Rib: $R e=55,283$ (Source: Fodemski and Collins 1988) 


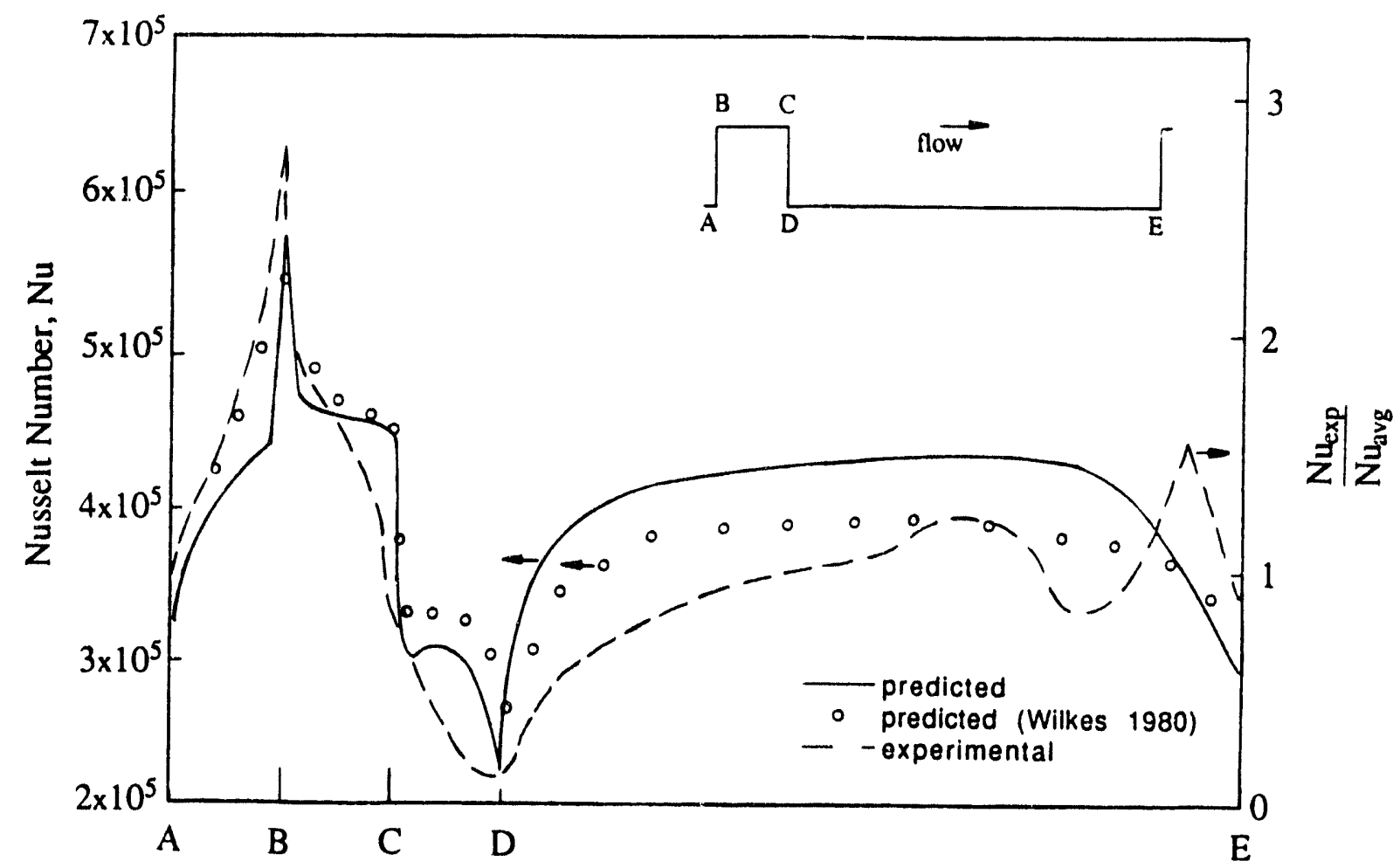

FIGURE 3.2 Nusselt Number for the Eighth Heated Rib: $R e=55,283$ (Source: Fodemski and Collins 1988)

TEACH-2EF computer code and the SIMPLE solution algorithm were used as the framework for the analysis. All the computations were performed with a $60 \times 26$ nonuniform grid system.

The major conclusions from this study were as follows:

1. The pressure loss increases with increasing rib height and rib width.

2. The normalized centerline velocity is not affected by the Reynolds number.

3. The average Nusselt number increases with the rib height and the Reynolds number.

4. The local Nusselt number has a sharp maximum at the top, at the downstream edge of the rib, and near the reattachment point. 
5. The empiricai correlation of the average Nusselt number is developed in the following form:

$$
\mathrm{Nu}=0.036 \operatorname{Re}^{0.75}(1+2 w / D)^{0.01}(1+2 e / D)^{1.1}
$$

6. The shear stress increases in the recirculating zone with increasing rib height.

This study was very comprehensive. However, a major limitation was its restriction to a single rib in a uniform-flow field rather than a fully developed temperature and velocity flow field.

\subsubsection{Hijlkata of al. (1987)}

Hijikata et al. (1987) presented a very short description of a numerical simulation of a fully developed flow in a rectangular channel (aspect ratio of 6) with 12 pairs of disruptions, $10 \mathrm{~mm}$ in height (one-fifth the channel height), at intervals of $150 \mathrm{~mm}(p / \mathrm{e}=15)$. An arc-shaped disruption was selected. Measurements of the flow field - velocities and turbulence properties - were also taken for comparison with the numerical predictions.

An improved $k-\varepsilon$ model was used that prevented the dissipation rate of the turbulent energy and the vorticity from becoming infinite at the wall. New functions were selected to equal zero at the wall and join with the results of the $k-\varepsilon$ model at the edge of the log-law region. Very few additional details of this two-layer model were supplied in the publication.

Comparisons of predicted and measured results were made. Acceptable agreement was obtained for the turbulent energy profiles and the mean heat-transfer coefficient, but the mean velocity profiles were underpredicted. The authors claimed that the major reasons for the discrepancies were that the logarithmic velocity profile near the reattachment point was not used, and that the turbulent-energy generation due to nonisotropy of the turbulence was not considered.

\subsubsection{Fujlta ot al. (1986)}

Fujita et al. (1986) performed numerical computations dealing with fully developed flow in a 50.0-mm square passage with two enhanced opposite walls. The square ribs were $1.0 \mathrm{~mm}$ high and placed at $10.0-\mathrm{mm}$ intervals $(p / \mathrm{e}=10)$. The selected Reynolds number value was 65,000 , and the test fluid was air. A constant wall-temperature boundary condition was imposed on both the enhanced and plain sides.

The prediction method employed a vorticity-stream function presentation for the solution in the direction orthogonal to the flow coupled with a marching procedure in the flow direction. Closure was obtained with an algebraic-stress model used in conjunction with $k$ or $k-\varepsilon$ models as first used by Launder and Ying (1972). 
The emphasis of the study was on the fluid mechanics. The study results showed only four streamwise vortices rather than the eight observed by experiment. The axial velocity and turbulent kinetic-energy predictions were in close agreement with the experimental results. The calculated temperature distribution and wall-heat flux were similar to the mean axial-velocity distribution and the wall shear stress, respectively. Direct comparisons between predicted and experimental temperature and wall heat-flux results were not made.

\subsubsection{Barba (1984)}

Barba (1984) presented the results of a very ambitious undertaking to optimize the flute geometry - the number or starts, size, angle, and shape - of a spirally fluted tube. A threedimensional analysis was required because of the swirl component that now existed in the flow field. Of particular interest were the number of starts and the helix angle. Flutes with a sinusoidal profile were examined with the local radius being $R_{w}=R_{m}+e / 2 \cos \left(2 p \alpha / \alpha^{\prime}\right)$, where $R_{m}$ is the mean radius; $e$ is the flute depth; $\alpha$ is the helix angle; and $\alpha^{\prime}$ is the sector angle, or $\alpha^{\prime}=2 p / \mathrm{n}$, where $\mathrm{n}$ is the number of flutes. Three fluids were considered: air, water, and $30 \%$ calcium chloride solution $(\operatorname{Pr}=19.6)$.

The computational procedure is based on the curvilinear-orthogonal TEACH code, which incorporates a finite-volume discretization of the clescribing partial differential equations. The turbulent modeling method employed the $k-\varepsilon$ Boussinesq viscosity model over most of the tube cross section, and it used the Van Driest's form of the mixing-length hypothesis in the near-wall region. The equations were solved with the PISO algorithm. The convergence rates depended greatly on the number of flutes and the Reynolds number - the rates were much faster for a smaller number of flutes and somewhat slower for larger Reynolds number values.

\subsubsection{Patankar et al. (1979)}

Patankar et al. (1979) performed the first numerical analysis to predict the turbulent heattransfer and pressure-drop performance in enhanced passages. They modeled the fully developed flow in circular and annular passages with longitudinal fins and with uniform heat input. At any axial location, the variation of the temperature along the fin height and around the circumference of the tube was neglected; however, this wall temperature varied axially in step with the increase of the bulk fluid temperature. Only the axial velocity component was considered, and no secondary flows or circulation between the fins was assumed to exist. The selected fluid was air.

Longitudinal finned enhancements have been identified by Rabas (1989) as not being energy-efficient. The enhancement is simply the result of additional surface area, and there is no similarity between this simple mechanism and that of the separation and reattachment enhancement mechanism. Although further discussion of this analysis is not presented in this report, it must be acknowledged as the first attempt to predict the turbulent heat-transfer performance in enhanced passages. 


\section{Governing Equations and Modeling Approach}

Sections 2 and 3 described the need to resolve the shape and Prandtl number issues and reviewed the progress of previous numerical modeling efforts with similar configurations. This section describes the governing equations, as well as the modeling approach that was selected and used to resolve these issues for fully developed pipe flow. Two computer programs were developed: the first prc ram was an upgraded version of an existing two-dimensional orthogonal code, and the second program was a completely new two-dimensional curvilinear coordinate code. The first program was used to explain the Prandtl number effect, and the second program was necessary to evaluate the shape effects. Both programs have the following common features:

- Conservation equations,

- Near-wall treatment,

- Boundary conditions, and

- Turbulent Prandtl number, $\operatorname{Pr}_{1}$.

The governing equations include the continuity, momentum, and energy equations, and the $k$ and $\varepsilon$ equations are used for closure. The near-wall treatment needed for the $k-\varepsilon$ near-wall closure, the selected near-wall two-equation model of Lam and Bremhorst (1981), and the twolayer model of Chen and Patel (1988) are described next. The boundary conditions are discussed for fully developed periodic in-tube flow. The selected turbulent heat-transfer closure criteria are reviewed in the last section.

\subsection{Conservation Equations}

The restrictions of the analysis are an incompressible fluid with a two-dimensional axisymmetric, fully developed, turbulent flow field. The starting point for the development of the governing equations is the conservation equations for an axisymmetric coordinate system, where $\mathrm{x}$ and $r$ denote the axial and radial directions, respectively:

$$
\begin{aligned}
& \frac{\partial U}{\partial x}+\frac{1}{r} \frac{\partial(r V)}{\partial r}=0 \\
& \rho\left(\frac{\partial U}{\partial t}+U \frac{\partial U}{\partial x}+V \frac{\partial U}{\partial r}\right)=-\frac{\partial P}{\partial x}+\frac{\partial}{\partial x}\left(2 \mu \frac{\partial U}{\partial x}\right)+\frac{1}{r} \frac{\partial}{\partial r} r\left[\mu\left(\frac{\partial U}{\partial r}+\frac{\partial V}{\partial x}\right)\right]
\end{aligned}
$$




$$
\begin{aligned}
& \rho\left(\frac{\partial \mathrm{V}}{\partial \mathrm{t}}+\mathrm{U} \frac{\partial \mathrm{V}}{\partial \mathrm{x}}+\mathrm{V} \frac{\partial \mathrm{V}}{\partial \mathrm{r}}\right)=-\frac{\partial P}{\partial \mathrm{r}}+\frac{\partial}{\partial \mathrm{x}}\left[\mu\left(\frac{\partial \mathrm{U}}{\partial \mathrm{r}}+\frac{\partial \mathrm{V}}{\partial \mathrm{x}}\right)\right]+\frac{1}{\mathrm{r}} \frac{\partial}{\partial \mathrm{r}} \mathrm{r}\left(2 \mu \frac{\partial \mathrm{V}}{\partial \mathrm{r}}\right)-\frac{2 \mu \mathrm{V}}{\mathrm{r}} \\
& \rho\left(\frac{\partial T}{\partial \mathrm{t}}+\mathrm{U} \frac{\partial T}{\partial \mathrm{x}}+\mathrm{V} \frac{\partial T}{\partial \mathrm{r}}\right)=\frac{\partial}{\partial \mathrm{x}}\left(\Gamma \frac{\partial T}{\partial \mathrm{x}}\right)+\frac{1}{\mathrm{r}} \frac{\partial}{\partial \mathrm{r}} \mathrm{r}\left(\Gamma \frac{\partial T}{\partial \mathrm{r}}\right)
\end{aligned}
$$

where:

$$
\begin{aligned}
& U=\text { instantaneous axial velocity component, } \\
& V=\text { instantaneous radial velocity component, } \\
& \rho=\text { density, } \\
& t=\text { time, } \\
& P=\text { instantaneous pressure, } \\
& \mu=\text { molecular viscosity, } \\
& T=\text { instantaneous temperature, and } \\
& \Gamma=\text { thermal diffusion coefficient. }
\end{aligned}
$$

The most common method of working with the conservation equations for steady-state turbulent analyses is to use time averaging. The time scale used for the averaging has long been compared with that of the turbulent motion. The instantaneous values of the velocities, pressure, and temperature are decomposed into time-averaged, or mean, and fluctuating parts, as written below:

$$
\begin{aligned}
& \mathrm{U}(\mathrm{x}, \mathrm{r}, \mathrm{t})=\mathrm{u}(\mathrm{x}, \mathrm{r})+\mathrm{u}^{\prime}(\mathrm{x}, \mathrm{r}, \mathrm{t}) \\
& \mathrm{V}(\mathrm{x}, \mathrm{r}, \mathrm{t})=\mathrm{v}(\mathrm{x}, \mathrm{r})+\mathrm{v}^{\prime}(\mathrm{x}, \mathrm{r}, \mathrm{t}) \\
& \mathrm{W}(\mathrm{x}, \mathrm{r}, \mathrm{t})=\mathrm{w}(\mathrm{x}, \mathrm{r})+\mathrm{w}^{\prime}(\mathrm{x}, \mathrm{r}, \mathrm{t}) \\
& P(\mathrm{x}, \mathrm{r}, \mathrm{t})=\mathrm{p}(\mathrm{x}, \mathrm{r})+\mathrm{p}^{\prime}(\mathrm{x}, \mathrm{r}, \mathrm{t})
\end{aligned}
$$




$$
T(\mathrm{x}, \mathrm{r}, \mathrm{t})=\mathrm{T}(\mathrm{x}, \mathrm{r})+\mathrm{T}^{\mathrm{y}}(\mathrm{x}, \mathrm{r}, \mathrm{t})
$$

where:

$$
\begin{aligned}
& u^{\prime}=\text { time-averaged axial velocity component, } \\
& u^{\prime}=\text { fluctuating axial velocity component, } \\
& v^{\prime}=\text { time-averaged radial velocity component, } \\
& v^{\prime}=\text { fluctuating radial velocity component, } \\
& w=\text { time-averaged angular velocity component } \\
& W^{\prime}=\text { instantaneous angular velocity component, } \\
& w^{\prime}=\text { fluctuating angular velocity component, } \\
& p^{\prime}=\text { time-averaged pressure, } \\
& p^{\prime}=\text { fluctuating pressure, } \\
& T=\text { time-averaged temperature, and } \\
& T^{\prime}=\text { fluctuating temperature. }
\end{aligned}
$$

The appropriate substitutions of Equations 4.2a through 4.2e into Equations 4.1a through 4.1d and subsequent time averaging gives the following steady-state conservation equations:

$$
\begin{aligned}
& \frac{\partial u}{\partial x}+\frac{1}{r} \frac{\partial(r v)}{\partial r}=0 \\
& \rho\left(\frac{\partial u}{\partial x}+v \frac{\partial u}{\partial r}\right)=-\frac{\partial p}{\partial x}+\frac{\partial}{\partial x}\left(2 \mu \frac{\partial u}{\partial x}-\rho \overline{u^{\prime 2}}\right)+\frac{1}{r} \frac{\partial}{\partial r} r\left[\mu\left(\frac{\partial u}{\partial r}+\frac{\partial v}{\partial x}\right)-\rho \overline{u^{\prime} v^{\prime}}\right] \\
& \rho\left(\frac{\partial v}{\partial x}+v \frac{\partial v}{\partial r}\right)=-\frac{\partial p}{\partial r}+\frac{\partial}{\partial x}\left[\mu\left(\frac{\partial u}{\partial r}+\frac{\partial v}{\partial x}\right)-\rho \overline{u^{\prime} v^{\prime}}\right]+\frac{1}{r} \frac{\partial}{\partial r} r\left(2 \mu \frac{\partial v}{\partial r}-\rho \overline{v^{2}}\right)+\rho \frac{w^{\prime 2}}{r}
\end{aligned}
$$




$$
\rho\left(u \frac{\partial T}{\partial x}+v \frac{\partial T}{\partial r}\right)=\frac{\partial}{\partial x}\left(\Gamma \frac{\partial T}{\partial x}-\rho \overline{u^{\prime} T^{\prime}}\right)+\frac{1}{r} \frac{\partial}{\partial r} r\left(\Gamma \frac{\partial T}{\partial r}-\rho \overline{v^{\prime} T^{\prime}}\right)
$$

The time-averaged momentum equations are called the Reynolds equations and contain new correlation terms of the fluctuating quantities, called the Reynolds stresses: $\overline{u^{2}}, \overline{u^{\prime} v^{\prime}}, \frac{v^{2}}{}$, and $\frac{w^{2}}{w^{2}}$. Also note the cross-correlation terms between the velocity and temperature fluctuations, $\overline{u^{\prime} \mathrm{T}^{\prime}}$ and $\overline{v^{\prime} T^{\prime}}$, in the energy equation (Equation 4.3d). To obtain closure for this equation set, the Reynolds stress terms and the velocity-temperature fluctuation terms must be modeled.

The $k-\varepsilon$ model was selected for closure of the above equation set. It is the most widely used model in engineering applications because of its simplicity, although it does suffer some limitations. The major limitations are due to the eddy-viscosity concept and the assumption of isotropy. The former implies that the turbulence correlation term is represented by the gradient of the appropriate time-averaged term and a new viscosity term called the eddy diffusivity, $v_{t}$. The Reynolds stresses are related to the corresponding mean rates of strain through an isotropic eddy diffusivity, $v_{t}$. The velocity-temperature correlations are related to the time-averaged temperature gradients and a thermal eddy diffusivity, $\alpha_{t}$. These equations are written as:

$$
\begin{aligned}
& -\overline{u^{\prime} u^{\prime}}=2 v_{t} \frac{\partial u}{\partial x}-\frac{2}{3} k \\
& -\overline{v^{\prime} v^{\prime}}=2 v_{t} \frac{\partial v}{\partial r}-\frac{2}{3} k \\
& -\overline{w^{\prime} w^{\prime}}=2 v_{t} \frac{v}{r}-\frac{2}{3} k \\
& -\overline{u^{\prime} v^{\prime}}=v_{t}\left(\frac{\partial v}{\partial x}+\frac{\partial u}{\partial r}\right) \\
& -\overline{u^{\prime} T^{\prime}}=\alpha_{t} \frac{\partial T}{\partial x} \\
& -\overline{v^{\prime} T^{\prime}}=\alpha_{t} \frac{\partial T}{\partial r}
\end{aligned}
$$


where $\mathrm{k}$ is the turbulent kinetic energy, which is defined as:

$$
k=\frac{1}{2}\left(\overline{u^{\prime 2}}+\overline{v^{\prime 2}}+\overline{w^{\prime 2}}\right)
$$

The turbulent eddy diffusivity will be described further in the discussion of the near-wall turbulence modeling. After the appropriate substitutions of Equations $4.4 \mathrm{a}$ through $4.4 \mathrm{~g}$ into Equations 4.3a through 4.3d, the conservation equations become:

$$
\begin{aligned}
& \frac{\partial \mathrm{u}}{\partial \mathrm{x}}+\frac{1}{\mathrm{r}} \frac{\partial(\mathrm{rv})}{\partial \mathrm{r}}=0 \\
& \rho\left(\frac{\partial \mathrm{u}}{\partial \mathrm{x}}+\mathrm{v} \frac{\partial \mathrm{u}}{\partial \mathrm{r}}\right)=-\frac{\partial \mathrm{p}}{\partial \mathrm{x}}+\frac{\partial}{\partial \mathrm{x}}\left(2 \mu_{\mathrm{e}} \frac{\partial \mathrm{u}}{\partial \mathrm{x}}\right)+\frac{1}{\mathrm{r}} \frac{\partial}{\partial \mathrm{r}} \mathrm{r}\left[\mu_{\mathrm{e}}\left(\frac{\partial \mathrm{u}}{\partial \mathrm{r}}+\frac{\partial \mathrm{v}}{\partial \mathrm{x}}\right)\right] \\
& \rho\left(\frac{\partial \mathrm{v}}{\partial \mathrm{x}}+\mathrm{v} \frac{\partial \mathrm{v}}{\partial \mathrm{r}}\right)=-\frac{\partial \mathrm{p}}{\partial \mathrm{r}}+\frac{\partial}{\partial \mathrm{x}}\left[\mu_{\mathrm{e}}\left(\frac{\partial \mathrm{u}}{\partial \mathrm{r}}+\frac{\partial \mathrm{v}}{\partial \mathrm{x}}\right)\right]+\frac{1}{\mathrm{r}} \frac{\partial}{\partial \mathrm{r}} \mathrm{r}\left(2 \mu_{\mathrm{e}} \frac{\partial \mathrm{v}}{\partial \mathrm{r}}\right)-2 \mu_{\mathrm{e}} \frac{\mathrm{v}}{\mathrm{r}} \\
& \rho\left(\frac{\partial \mathrm{T}}{\partial \mathrm{x}}+\mathrm{v} \frac{\partial \mathrm{T}}{\partial \mathrm{r}}\right)=\frac{\partial}{\partial \mathrm{x}}\left(\Gamma_{\mathrm{e}} \frac{\partial \mathrm{T}}{\partial \mathrm{x}}\right)+\frac{1}{\mathrm{r}} \frac{\partial}{\partial \mathrm{r}} \mathrm{r}\left(\Gamma_{\mathrm{e}} \frac{\partial \mathrm{T}}{\partial \mathrm{r}}\right)
\end{aligned}
$$

where $\mu_{e}, \mu_{e}=\mu+\mu_{t}$, is the effective viscosity, and $\Gamma_{c}, \Gamma_{c}=\Gamma+\Gamma_{l}$, is the effective therrnaldiffusion coefficient.

These equations can be solved for $u, v, p$, and $T$ when suitable turbulence models are employed for the momentum and thermal eddy-diffusivity distributions. In the $k-\varepsilon$ model, the momentum eddy viscosity, $\mu_{t}$, is related to the turbulent kinetic energy, $k$, and the dissipation, $\varepsilon$, by the expression:

$$
\mu_{t}=\rho C_{\mu} f_{\mu} \frac{k^{2}}{\varepsilon}
$$

The $k$ and $\varepsilon$ are obtained from the models of their transport equations. The modeled transport equation for the turbulent kinetic energy is:

$$
\rho\left(u \frac{\partial k}{\partial x}+v \frac{\partial k}{\partial r}\right)=\frac{\partial}{\partial x}\left[\left(\mu+\frac{\mu_{t}}{\sigma_{k}}\right) \frac{\partial k}{\partial x}\right]+\frac{1}{r} \frac{\partial}{\partial r} r\left[\left(\mu+\frac{\mu_{t}}{\sigma_{k}}\right) \frac{\partial k}{\partial r}\right]+G-\rho \varepsilon
$$


where $\mu_{\mathrm{t}}$ is the turbulent viscosity, and $\mathrm{G}$ is the turbulent kinetic energy generation term:

$$
G=\mu_{t}\left\{2\left[\left(\frac{\partial u}{\partial x}\right)^{2}+\left(\frac{\partial u}{\partial x}\right)^{2}\right]+\left(\frac{\partial u}{\partial r}+\frac{\partial v}{\partial x}\right)^{2}+2 \frac{v^{2}}{r^{2}}\right\}
$$

The modeled transport equation for the turbulent energy dissipation is:

$$
\rho\left(u \frac{\partial \varepsilon}{\partial x}+v \frac{\partial \varepsilon}{\partial r}\right)=\frac{\partial}{\partial x}\left[\left(\mu+\frac{\mu_{t}}{\sigma_{\varepsilon}}\right) \frac{\partial \varepsilon}{\partial x}\right]+\frac{1}{r} \frac{\partial}{\partial r} r\left[\left(\mu+\frac{\mu_{t}}{\sigma_{\varepsilon}}\right) \frac{\partial \varepsilon}{\partial r}\right]+\frac{\varepsilon}{k}\left(C_{\varepsilon 1} f_{\varepsilon 1} G-C_{\varepsilon 2} f_{\varepsilon 2} \varepsilon\right)
$$

The constants in equations $4.6 a$ through $4.6 \mathrm{c}$ are as follows: $\mathrm{C}_{\mu}=0.09, \mathrm{C}_{\varepsilon 1}=1.44, \mathrm{C}_{\varepsilon 2}=1.92$, $\sigma_{k}=1.0$, and $\sigma_{\varepsilon}=1.3$. In ths above models, $f_{\mu}, f_{\varepsilon 1}$, and $f_{\varepsilon 2}$ are wall-damping functions that must be prescribed in equations $4.6 a\left(f_{\mu}\right)$ and $4.6 \mathrm{~d}\left(f_{\varepsilon 1}\right.$ and $\left.f_{\varepsilon 2}\right)$. Seven equations now exist to calculate the seven unknowns: $u, v, p, T, \mu_{t}, k$, and $\varepsilon$. One unknown still remains: the thermal eddy diffusivity, $\alpha_{\mathfrak{l}}$, or thermal eddy diffusion coefficient, $T_{l}=\rho \alpha_{\mathfrak{l}}$, that will be discussed later.

\subsection{Near-Wall Turbulence Modeling}

The conservation equations coupled with the $k-\varepsilon$ method is used only in the fully turbulent region some distance beyond the tube wall. Near the wall, modifications to this $k-\varepsilon$ model are necessary to account for the viscous damping. Various near-wall $k-\varepsilon$ two-equation models have been developed. Examples are listed below.

- Two-equation models with modified wall functions

- Patel (1973)

- Chieng and Launder (1980)

- Amano (1983)

- Ciofalo and Collins (1989)

- Low-Reynolds-number two-equation models

- Launder and Sharma (1974) 
- Lam and Bremhorst (1981)

- Chien (1982)

- Myong and Kasagi (1990)

- Nagano and Tagawa (1990)

- Two-layer model, Chen and Patel (1988)

The initial approach - at the beginning of this project - was to use standard and/or modified wall-function models. As the work proceeded, the limitations of wall-function methods became more apparent, and the preferred choice shifted to the low-Reynolds-number models. The low-Reynolds-number two-equation models and the two-layer model were incorporated into the orthogonal numerical code. The majority of the low-Reynolds-number models employ the friction velocity; however, the friction velocity changes sign when the flow separates. As a result, the use of the friction velocity concept is being questioned by Chen and Patel (1988), Rodi (1991), and Arman and Rabas (1991) for separated flows. The validity of this point is shown by further numerical studies. It was concluded that the appropriate low-Reynolds-number model is that of Lam and Bremhorst (1981). The advantages of this model are two-fold: (1) it does not employ the friction velocity, and (2) it does not require additional terms in the governing equations.

The two-layer model also does not use the wall-friction velocity; it uses a near-wall treatment that is dependent only on the local turbulence intensity. This model exhibits an additional advantage: only the turbulent kinetic-energy equation needs to be solved in the near-wall region. This leads to better convergence and permits the use of a coarser grid. Consequently, the use of the two-layer model is preferred; however, the low-Reynolds-number two-equation model of Lam and Bremhorst (1981) is also used.

\subsubsection{Low-Reynolds-Number Two-Equation Model of Lam and Bremhorst (1981)}

The wall-damping functions are:

$$
\begin{aligned}
& f_{\mu}=\left[1-\exp \left(-0.0160 R e_{y}\right)\right]^{2}\left(1+\frac{19.5}{R e_{t}}\right) \\
& f_{\varepsilon 1}=1+\left(\frac{0.06}{f_{\mu}}\right)^{3} \\
& f_{\varepsilon 2}=1-\exp \left(-R_{t}^{2}\right)
\end{aligned}
$$


where $\mathrm{Re}_{\mathrm{y}}$ and $\mathrm{Re}_{\mathrm{t}}$ are the turbulence Reynolds numbers:

$$
\begin{aligned}
& \operatorname{Re}_{y}=y \sqrt{k} \rho / \mu \\
& \operatorname{Re}_{t}=\frac{\rho k^{2}}{\mu \varepsilon}
\end{aligned}
$$

where $y$ is the distance from the wall.

\subsubsection{Two-Layer Model of Chen and Patel (1988)}

This section presents the governing equations of this model that are derived from Chen and Patel (1988). The flow field is divided into two regions: a near-wall region that includes the sublayer, the buffer layer, and a part of the turbulent core and a fully turbulent core region. The standard $\mathrm{k}-\varepsilon$ model described in detail above is used in the turbulent core region. In the near-wall region, only the turbulent kinetic energy solution is obtained. The near-wall damping coefficients, $f_{\mu}, f_{\varepsilon}, f_{\varepsilon 2}$, are set to unity. The energy dissipation in this region is specified by:

$$
\varepsilon=\left(\mathrm{k}^{2 / / l_{\varepsilon}}\right)
$$

where the eddy diffusivity is obtained from:

$$
v_{\mathrm{t}}=\mathrm{C}_{\mu} \mathrm{k}^{1 / 2} l_{\mu}
$$

The length scales, $l_{\varepsilon}$ and $l_{\mu}$, contain the necessary damping effects in the near-wall region and are defined below:

$$
\begin{aligned}
& l_{\varepsilon}=C_{l} y\left[1-\exp \left(-\operatorname{Re}_{y} / A_{\varepsilon}\right)\right] \\
& l_{\mu}=C_{l} y\left[1-\exp \left(-\operatorname{Re}_{y} / A_{\mu}\right)\right]
\end{aligned}
$$

where $C_{l}, A_{\varepsilon}$, and $A_{\mu}$ are empirical constants.

Note that both $l_{\varepsilon}$ and $l_{\mu}$ become linear and approach $\mathrm{C}_{l} \mathrm{y}$ with increasing distance from the wall. $C_{l}$ is given by:

$$
\mathrm{C}_{l}=\kappa \mathrm{C}_{\mu}^{-3 / 4}
$$


to ensure a smooth eddy-diffusivity distribution at the junction of the near-wall and fully turbulent core regions. In addition, $A_{\varepsilon}=2 C_{l}$ or is assigned a variation so as to recover the proper asymptotic behavior of the kinetic energy in the sublayer:

$$
\varepsilon=2 \vee \mathrm{k} / \mathrm{y}^{2}
$$

where $v$ is the molecular diffusivity.

The parameter, $A_{\mu}=70$, is determined from numerical results to recover the additive constant, $B=5.45$, in the velocity $\log$ law (i.e., $u^{+}=1 / \kappa \ln \left[y^{+}\right]+B$ ) in the case of a near-wall boundary-layer flow field.

The wall shear stress does not appear in this formulation and, in particular, in the Reynolds number, $R_{e}$. The Reynolds number $\operatorname{Re}_{y}$ varies slowly along lines parallel to the wall, does not vanish at the separation point, and remains well defined in regions of flow reversal. As a result, damping effects decay rapidly with the distance from the wall regardless of the magnitude of the wall shear stress. The matching between the the one-equation near-wall treatment and the twoequation standard $k-\varepsilon$ model in the two-layer approach can be carried out along preselected grid lines, even for complex flows with separation. For this application, the match boundary was selected along a grid line with a $y^{+}$in the range of $100-200$, or far from the near-wall region, to ensure a smooth eddy-diffusivity distribution across the boundary of the two regions. Recently, Patel and his coworkers tried a constant match boundary corresponding to $\operatorname{Re}_{y}=200$. Their findings indicated that there is no significant change in the results (Patel 1992).

\subsection{Boundary Conditions}

The tube geometry consists of transverse, repeated disruptions as shown in Figure 1.1. Only one rib pitch with appropriate periodicity conditions is considered because the flow and heattransfer fields are fully developed. Boundary conditions are required on the wall, at the centerline, and at the inlet $(\mathrm{x}=0)$ and outlet $(\mathrm{x}=p)$.

\subsubsection{Wall and Centerline Boundary Conditions}

The boundary conditions at the wall are:

$$
\begin{aligned}
& \mathrm{u}=\mathrm{v}=\mathrm{k}=0 \\
& \frac{\partial \mathrm{T}}{\partial \mathrm{n}}=-\frac{\dot{\mathrm{q}}_{\mathrm{w}}}{k}
\end{aligned}
$$


where $\dot{q}_{w}$ is the wall heat flux, and $k$ is the thermal conductivity.

For the Lam and Bremhorst two-equation model, an additional boundary condition for the turbulent energy dissipation is needed at the wall. Patel et al. (1985) suggested the following:

$$
\frac{\partial \varepsilon}{\partial \mathrm{n}}=0
$$

The boundary conditions on the pipe axis are the usual symmetry conditions, that is:

$$
\frac{\partial u}{\partial n}=\frac{\partial v}{\partial n}=\frac{\partial k}{\partial n}=\frac{\partial \varepsilon}{\partial n}=\frac{\partial T}{\partial n}=0
$$

\subsubsection{Cyclic Boundary Conditions}

As discussed in Section 3, the previous numerical heat-transfer investigations of enhanced passages dealt with a prescribed or developing flow and temperature fields upstream of the disruption. Previous studies did not address the fully developed turbulent heat-transfer analysis with transverse disruptions, regardless of the passage shape. However, the fully developed case is the most important because it exists for almost all heat-exchanger applications. This section reviews the wrap-around procedure that was developed to deal with fully developed turbulent flow and temperature fields. The basis for this development was the publications of Patankar et al. (1977) and Sparrow and Prata (1983).

At steady-state conditions, a fully developed velocity field for a periodic enhancement geometry - the normal case - must satisfy the conditions:

$$
\begin{aligned}
& \mathrm{u}(\mathrm{x}, \mathrm{r})=\mathrm{u}(\mathrm{x}+p, \mathrm{r})=\mathrm{u}(\mathrm{x}+2 p, \mathrm{r})=\cdots \\
& \mathrm{v}(\mathrm{x}, \mathrm{r})=\mathrm{v}(\mathrm{x}+p, \mathrm{r})=\mathrm{v}(\mathrm{x}+2 p, \mathrm{r})=\cdots \\
& \mathrm{k}(\mathrm{x}, \mathrm{r})=\mathrm{k}(\mathrm{x}+p, \mathrm{r})=\mathrm{k}(\mathrm{x}+2 p, \mathrm{r})=\cdots \\
& \varepsilon(\mathrm{x}, \mathrm{r})=\varepsilon(\mathrm{x}+p, \mathrm{r})=\varepsilon(\mathrm{x}+2 p, \mathrm{r})=\cdots
\end{aligned}
$$

or identical distributions but at increments of $p$ or one pitch. These equations also specify the inlet and outlet boundary conditions at $\mathrm{x}=0$ and $\mathrm{x}=p$. The periodicity is the direct result of the fully developed flow-field requirement. 
A pressure gradient must be present for axial flow to exist. As a result, the pressure cannot be identical and must follow a different periodicity requirement for fully developed flow, which is as follows:

$$
\mathrm{p}(\mathrm{x}, \mathrm{r})-\mathrm{p}(\mathrm{x}+p, \mathrm{r})=\mathrm{p}(\mathrm{x}+p, \mathrm{r})-\mathrm{p}(\mathrm{x}+2 p, \mathrm{r})=\cdots
$$

This pressure-difference value is responsible for the mass flux in the axial direction. Define the quantity, $\Delta \mathrm{p}$, where:

$$
\Delta \mathrm{p} / p=[\mathrm{p}(\mathrm{x}, \mathrm{r})-\mathrm{p}(\mathrm{x}+p, \mathrm{r})] / p
$$

As a result, the local pressure is divided into two parts:

$$
\mathrm{p}(\mathrm{x}, \mathrm{r})=-\Delta \mathrm{p}(\mathrm{x} / \mathrm{p})+\mathrm{P}(\mathrm{x}, \mathrm{r})
$$

where the first term on the right-hand side is related to the total flow rate, and $\mathrm{P}(\mathrm{x}, \mathrm{r})$ is related to only the local flow characteristics and must satisfy the periodicity conditions:

$$
\mathrm{P}(\mathrm{x}, \mathrm{r})=\mathrm{P}(\mathrm{x}+p, \mathrm{r})=\mathrm{P}(\mathrm{x}+2 p, \mathrm{r})=\cdots
$$

This substitution, $P(x, r)$ for $p(x, r)$, adds an additional term in the u-momentum equation, $\Delta \mathrm{p} / p$, but does not alter the continuity and $\mathrm{v}$-momentum equation. However, now $\mathrm{u}(\mathrm{x}, \mathrm{r}), \mathrm{v}(\mathrm{x}, \mathrm{r})$, and $P(x, r)$ are periodic for one axial pitch length.

The magnitude of the periodic pressure difference, $\Delta p$, for one pitch must be specified to run the program. The program then calculates the flow rate that exists for this pressure difference. This approach can be considered as a backward approach; the preferred approach is to specify the flow rate and then to calculate the pressure drop. The advantage of the wrap-around method is that only one disruption is considered and a very fine grid structure can be used. The other method is to use multiple disruptions and assume that the fully developed flow exists after an assumed number of disruptions. Patankar et al. (1979) present more discussion on the application of the wrap-around method.

A similar approach is required for a fully developed temperature field, but the details depend on the wall boundary condition. The constant heat-flux case is the boundary condition selected for this study. As with the pressure profiles, the fully developed temperature profiles must be identical at axial increments of one pitch except for a constant value equal to:

$$
\Delta \mathrm{T}=\frac{\mathrm{Q}}{\dot{\mathrm{m}} \mathrm{c}_{\mathrm{p}}}
$$


where $Q$ is the total heat added for one period or one pitch, $\dot{m}$ is the mass flow rate, $c_{p}$ is specific heat, and $\Delta \mathrm{T}$ is the bulk-average temperature rise following directly from a global heat balance. This fully developed requirement can be expressed as:

$$
\mathrm{T}(\mathrm{x}+p, \mathrm{r})-\mathrm{T}(\mathrm{x}, \mathrm{r})=\mathrm{T}(\mathrm{x}+2 p, \mathrm{r})-\mathrm{T}(\mathrm{x}+p, \mathrm{r})=\cdots=\mathrm{x} \mathrm{Q} /\left(\mathrm{m}_{\mathrm{p}}\right)
$$

Now define a new temperature, $\theta$, where:

$$
\theta(\mathrm{x}, \mathrm{r})=\mathrm{T}(\mathrm{x}, \mathrm{r})-\left(\mathrm{Q} / \mathrm{mc}_{\mathrm{p}}\right)(\mathrm{x} / \mathrm{p})-\mathrm{T}_{\mathrm{ref}}
$$

$\theta$ also is periodic similar to the pressure or:

$$
\theta(\mathrm{x}, \mathrm{r})=\theta(\mathrm{x}+p, \mathrm{r})=\theta(\mathrm{x}+2 p, \mathrm{r})=\cdots
$$

Substituting $\theta$ for $T$ in the energy equation introduces another term equal to $\mathrm{\rho u}(\mathrm{x}, \mathrm{r}) \mathrm{Q} /\left(\dot{\mathrm{m}} \mathrm{c}_{\mathrm{p}} p\right)$ on the left-hand side and $\mathrm{Q}\left(\dot{\mathrm{m}} \mathrm{c}_{\mathrm{p}} p\right)(\partial \theta / \partial \mathrm{x})$ on the right-hand side of the energy equation (4.5d):

$$
\rho\left(\mathrm{u} \frac{\partial \theta}{\partial \mathrm{x}}+\mathrm{v} \frac{\partial \theta}{\partial \mathrm{r}}\right)+\rho \mathrm{u} \frac{\mathrm{Q}}{\dot{\mathrm{m}} \mathrm{c}_{\mathrm{p}} p}=\frac{\partial}{\partial \mathrm{x}}\left(\Gamma_{\mathrm{e}} \frac{\partial \theta}{\partial \mathrm{x}}\right)+\frac{1}{\mathrm{r}} \frac{\partial}{\partial \mathrm{r}} \mathrm{r}\left(\Gamma_{\mathrm{e}} \frac{\partial \theta}{\partial \mathrm{r}}\right)+\frac{\mathrm{Q}}{\dot{\mathrm{m}}_{\mathrm{p} p} p} \frac{\partial \theta}{\partial \mathrm{x}}
$$

The boundary conditions for $\theta$ become the following:

$$
\frac{\partial \theta}{\partial \mathrm{n}}=-\frac{\dot{\mathrm{q}}_{\mathrm{w}}}{k}-\frac{\mathrm{Q}}{\dot{\mathrm{m}} \mathrm{c}_{\mathrm{p}} p} \frac{\partial \mathrm{x}}{\partial \mathrm{n}}
$$

and at the centerline:

$$
\frac{\partial \theta}{\partial \mathrm{n}}=-\frac{\mathrm{Q}}{\dot{\mathrm{m}} \mathrm{c}_{\mathrm{p}} p} \frac{\partial \mathrm{x}}{\partial \mathrm{n}}
$$

The wall heat flux, $\dot{q}_{w}$, is the prescribed local wall heat flux. The surface-area average of $\dot{q}_{w}$ for an axial tube length of one pitch is $Q$, which consists of contributions from the rib and the remaining smooth portion of the tube. It is therefore evident that 


$$
\left.Q=2 \pi \dot{q}_{w} \int_{0}^{w} r(x) \sqrt{1+\left(\frac{d r}{d x}\right.}\right) d x+\pi D \dot{q}_{w}(p-w)
$$

where $D$ is the maximum pipe inside diameter.

Note that the periodicity conditions are different for a constant wall temperature condition. Patankar et al. (1977) and Sparrow and Prata (1983) presented detailed descriptions for this condition.

\subsection{Turbulent Heat-Transfer Closure}

The heat transfer in the turbulent flow field is strongly affected by the local flow conditions. Because of the scarcity of local thermal turbulence measurements, it is often assumed that a similarity exists between the heat transfer and the momentum transport (Reynolds analogy and turbulent Prandtl number).

Current engineering practice is to use either the eddy diffusivity or turbulent heat-flux models. This study employs the eddy diffusivity concept for both momentum and heat transport. The thermal eddy diffusivity is defined in Equations $4.4 \mathrm{e}$ and $4.4 \mathrm{~d}$ and is purely a function of the local flow field.

Similar to the eddy diffusivity, $v_{t}$, the thermal diffusivity, $\alpha_{t}$, is proportional to the product of the local velocity, $\mathrm{U}_{\alpha}$, and length scale, $l_{\alpha}$.

$$
\alpha_{t} \sim l_{\alpha} U_{\alpha}
$$

In general, this length scale is different from those of the momentum diffusivity. However, the simplest and most common assumption is to use the same length scale as the momentum transport. Therefore:

$$
\alpha_{t} \sim v_{t}
$$

or:

$$
\operatorname{Pr}_{t}=\frac{v_{t}}{\alpha_{t}}
$$

where $\mathrm{Pr}_{t}$ is the turbulent Prandtl number and is analogous to the molecular Prandtl number. The turbulent Prandtl number is the only parameter to account for the difference between turbulent heat and momentum transport. 
A number of experiments and theoretical treatments have been used to determine $\operatorname{Pr}_{t}$ (Reynolds 1975). Even with these studies, a considerable amount of uncertainty regarding the magnitude of the turbulent Prandtl number still exists, which is especially amplified for large Prandtl number fluids. It is common practice in engineering calculations to assume that turbulent Prandtl number is constant or only a function of molecular Prandtl number. In this study, the following are used:

$$
\begin{array}{ll}
\mathrm{Pr}_{\mathrm{t}}=0.9 & \text { for } \mathrm{Pr}<1 \\
\mathrm{Pr}_{\mathrm{t}}=1.01-0.09 \mathrm{Pr}^{0.36} & \text { for } 1<\operatorname{Pr}<145
\end{array}
$$

where the last expression is attributable to Malhotra and Kang (1984). 


\section{Numerical Approach}

Two finite-difference numerical codes were used during this investigation. At the initial stage, a two-dimensional, axisymmetric, orthogonal finite-volume-method orthogonal code, based on Patankar and Spalding's SIMPLE approach, was employed (Mah 1987). This program was previously used for a direct-contact modeling research investigation. During the course of the work, a new two-dimensional, axisymmetric, nonorthogonal curvilinear numerical code - a body-fitted code - was developed. The two codes have many similarities; however, there are three distinct differences: (1) the orthogonal numerical code employs orthogonal Cartesian coordinates, whereas the body-fitted code employs nonorthogonal curvilinear coordinates; (2) the grid network of the orthogonal code is based on staggered meshes (Harlow and Welch 1965), whereas the grid network of the body-fitted code consists of colocated meshes, as shown in Figure 5.1 ; and (3) the body-fitted numerical code has a number of curvature-compensated convective differencing schemes in addition to the differencing schemes that are employed by the orthogonal program.

The orthogonal program runs were performed with the hybrid (Spalding 1972) and the QUICK (Leonard 1979; Phillips and Schmidt 1985a) schemes. The hybrid scheme is a blend of the first-order central differencing and the first-order upwind differencing schemes, where the appropriate approximation is chosen as a function of the so-called cell Peclet number, defined as the ratio of the convective and diffusive terms. The first-order approximate central or upwind schemes for the convection terms will cause numerical diffusion, especially when the convective terms dominate or the mesh network is relatively coarse. On the other hand, the hybrid scheme is unconditionally stable; thus, it is most often used during preliminary runs. The final runs were completed with the third-order-correct QUICK scheme. The following subsections discuss only the numerical aspects of the body-fitted code.

\subsection{Nonorthogonal, Body-Fitted Coordinates}

The complex disruption geometries shown in Figure 2.2 cannot conveniently be described by simple orthogonal coordinate systems. As a result, it has been necessary to introduce coordinate transformations to simplify the computational domain and thus the applications of the boundary conditions. The numerically generated, nonorthogonal, body-fitted coordinates offer three advantages: (1) the body-fitted grids match the boundaries of the physical domain, (2) the grid positions and clustering are easily accomplished, and (3) the problem of solving the governing equations on a complex domain is modified to solving the transformed equations in a simpler, rectangular region with equal grid spacing. The third advantage is the most important.

The basic idea of a body-fitted curvilinear system is as follows: the arbitrary domain (complex body shape with $\mathrm{x}$ and $\mathrm{r}$ coordinates) is transformed into a rectangular domain, where the 
Staggered Grid Layout

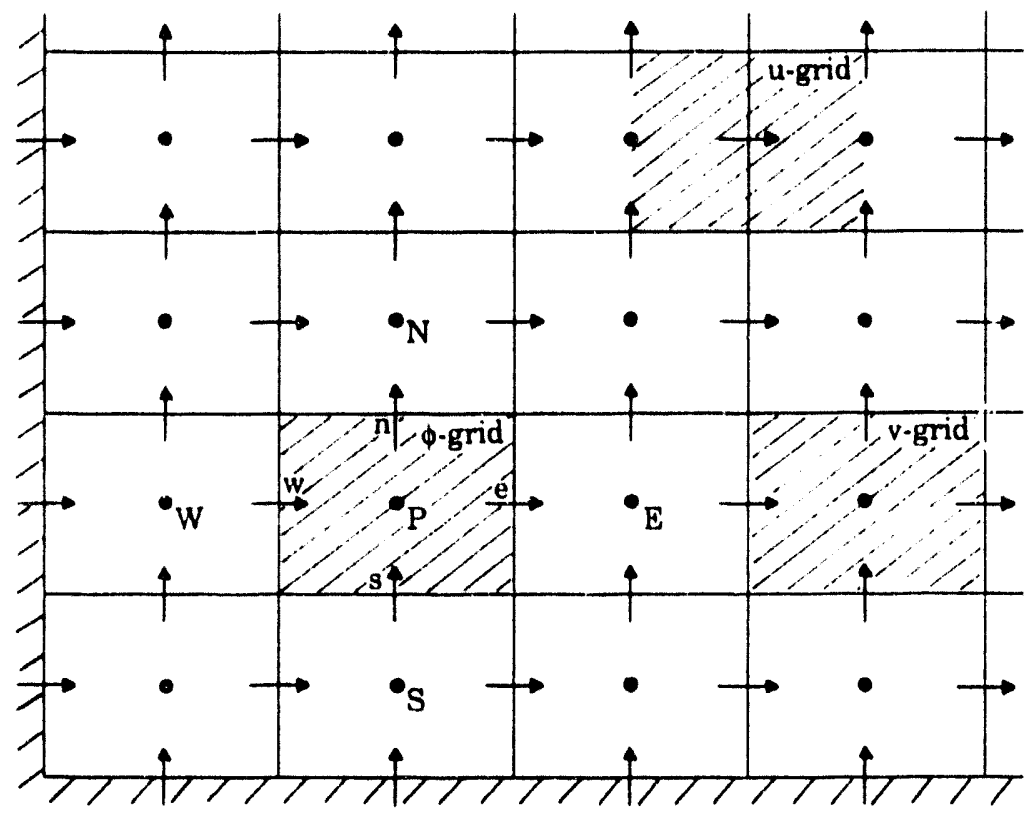

Colocated Grid Layout

Storage Locations

- p. T, p. $\mu$

$\uparrow v$

$\square \mathbf{C}_{\mathrm{x}}$

$0 \mathrm{C}_{\mathrm{y}}$

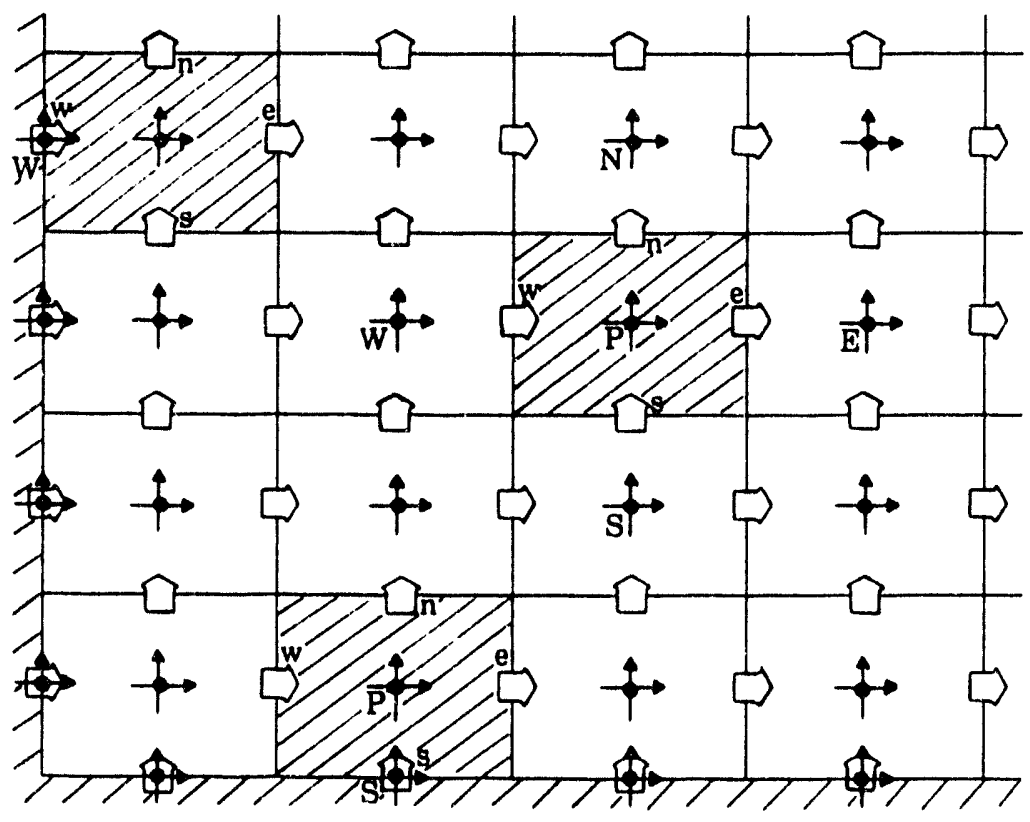

FIGURE 5.1 Staggered and Colocated Grid Layout 
independent variables are $\xi$ and $\eta$. In other words, the coordinates will be transformed according to the transformations:

$$
\begin{aligned}
& \xi=\xi(\mathrm{x}, \mathrm{r}) \text { and } \\
& \eta=\eta(\mathrm{x}, \mathrm{r}) \text {. }
\end{aligned}
$$

The boundaries of the physical domain are mapped to the transformed domain. Detailed discussions of the curvilinear coordinates and tensor analysis are contained in books by Aris (1962), Thompson (1982), and Thompson et al. (1985).

\subsection{Transformation of Governing Equations}

The complete set of governing equations in axisymmetric cylindrical coordinates can be cast into the following form:

$$
\frac{\partial}{\partial x}(\rho r u \phi)+\frac{\partial}{\partial r}(\rho r v \phi)=\frac{\partial}{\partial x}\left(r \Gamma^{\phi} \frac{\partial \phi}{\partial x}\right)+\frac{\partial}{\partial r}\left(r \Gamma^{\phi} \frac{\partial \phi}{\partial r}\right)+r S^{\phi}
$$

where $\phi$ is an arbitrary scalar variable, $\Gamma^{\phi}$ is an effective diffusion coefficient, and $\mathrm{S}^{\phi}$ denotes the various respective source terms. When the new independent variables $\xi$ and $\eta$ are introduced, Equation 5.2 changes according to the general transformation given by Equations 5.1a and 5.1b. Equation 5.2 and the continuity equation, Equation $4.5 \mathrm{a}$, are written in the transformed domain $(\xi, \eta)$ as follows:

$$
\begin{aligned}
\frac{\partial}{\partial \xi}\left(\operatorname{\rho r} U_{c} \phi\right) & +\frac{\partial}{\partial \eta}\left(\rho r V_{c} \phi\right)=\frac{\partial}{\partial \xi}\left[\frac{\mathrm{r} \Gamma^{\phi}}{\sqrt{g}}\left(g_{22} \phi_{\xi}-g_{12} \phi_{\eta}\right)\right] \\
+ & \frac{\partial}{\partial \eta}\left[\frac{\mathrm{r} \Gamma^{\phi}}{\sqrt{g}}\left(-g_{12} \phi_{\xi}+g_{11} \phi_{\eta}\right)\right]+r \sqrt{g} S^{\phi} \\
\frac{\partial}{\partial \xi}\left(\rho r U_{c}\right) & +\frac{\partial}{\partial \eta}\left(\rho r V_{c}\right)=0
\end{aligned}
$$


where:

$$
\begin{aligned}
& U_{c}=u y_{\eta}-v x_{\eta} \\
& V_{c}=v_{\xi}-u_{\xi}
\end{aligned}
$$

and the $g_{i j}$ 's and $\sqrt{g}$ are called the metric tensors and the Jacobian of the transformation, respectively. The metrics and the Jacobian of the transformation are as follows:

$$
\begin{aligned}
& g_{11}=x_{\xi}+y_{\xi} \\
& g_{22}=x_{\eta}+y_{\eta} \\
& g_{12}=x_{\xi} x_{\eta}+y_{\xi} y_{\eta} \\
& \sqrt{g}=x_{\xi} x_{\eta}-y_{\xi} y_{\eta} .
\end{aligned}
$$

Note that $g_{12}$ vanishes when the coordinates are orthogonal; thus, it can be interpreted as the degree of nonorthogonality of the local grid. The terms $U_{c} / \sqrt{g_{22}}$ and $V_{c} / \sqrt{g_{22}}$ represent the contravariant velocities (i.e., the velocity components normal to constant $\xi$ - and $\eta$-lines, respectively).

\subsection{Numerical Solution Procedure}

\subsubsection{Grid Generation}

With the values of the curvilinear coordinates specified on the boundaries of the transformed domain, values of the coordinate points in the interior of the domain can now be generated. This is a boundary-value problem in the physical domain with the body-fitted coordinates $(\xi, \eta)$ as dependent variables and the orthogonal coordinates $(x, r)$ as the independent variables. A pair of elliptic, partial-differential equations are used to generate the coordinate values in the interior from the values given on the boundaries. 
For the general two-dimensional but simply connected physical domain, a set of elliptic Poisson equations of the following form are solved:

$$
\begin{aligned}
& \nabla^{2} \xi=\mathrm{f}^{1}(\xi, \eta) \\
& \nabla^{2} \eta=\mathrm{f}^{2}(\xi, \eta)
\end{aligned}
$$

where $\nabla^{2}$ is the Laplacian operator in the orthogonal coordinates.

It is desirable to perform all of the numerical computations in the transformed plane $(\xi, \eta)$; thus, it is more convenient to invert the above equations and solve for the orthogonal coordinates (i.e., the dependent and independent variables are interchanged). Therefore:

$$
\begin{aligned}
& \nabla^{* 2} x=f^{* 1}(x, r) \\
& \nabla^{* 2} r=f^{* 2}(x, r)
\end{aligned}
$$

where:

$$
\nabla^{* 2}=g_{22} \frac{\partial}{\partial \xi \partial \xi}-2 g_{12} \frac{\partial}{\partial \xi \partial \eta}+g_{11} \frac{\partial}{\partial \eta \partial \eta}
$$

and the $\mathrm{g}_{\mathrm{ij}}$ 's are the metric tensors in the transformed coordinates $(\xi, \eta)$. These equations are solved numerically in the transformed domain with the proper boundary conditions specified on all of the boundary surfaces. If the control functions $\left(\mathrm{f}^{* 1}, \mathrm{f}^{*}\right)$ are equal to zero, the transformation will generate orthogonal body-fitted grids. In general, nonzero values are assigned to these functions to control the grid distribution and clustering.

The current control functions are:

$$
\begin{aligned}
& f^{* 1}=-\frac{g_{22}}{g} \frac{x_{\xi \xi}}{x_{\xi}} \\
& f^{* 2}=-\frac{g_{11}}{g} \frac{x_{\eta \eta}}{x_{\eta}} .
\end{aligned}
$$


These control functions employ prescribed boundary-point distributions. In addition to these functions, the program has more sophisticated exponential control functions, which allow the grid lines to attract to certain points and/or lines (see Thompson et al. 1985 for more details).

Equations $5.8 \mathrm{a}$ and $5.8 \mathrm{~b}$ are discretized by second-order central differences. The boundary points are also specified along the entire body surface. The discretized elliptic equations with the prescribed boundary conditions are solved by the well-known successive overrelaxation procedure.

\subsubsection{Discretization of the Governing Equations}

A colocated grid system is adopted and displayed in Figure 5.1. All of the dependent variables $(\mathrm{u}, \mathrm{v}, \mathrm{p}, \mathrm{T}, \mathrm{k}$, and $\varepsilon$ ) are located at the control-volume center point, $\mathrm{P}$, surrounded by $\mathrm{N}$, $S, E$, and $W$, while the velocities, $U_{c}$ and $V_{c}$, and corresponding fluxes, $C_{x}$ and $C_{y}$, are located at the cell faces ( $\mathrm{n}, \mathrm{s}, \mathrm{e}, \mathrm{w})$. Equation 5.3 can be discretized to give:

$$
\begin{aligned}
\left(\rho U_{c} \phi r \Delta \eta\right)_{w}^{e} & +\left(\rho V_{c} \phi r \Delta \xi\right)_{s}^{n}=\left[\frac{r \Gamma^{\phi}}{\sqrt{g}}\left(g_{22} \phi_{\xi}-g_{12} \phi_{\eta}\right)\right]_{w}^{e} \\
& +\left[\frac{r \Gamma^{\phi}}{\sqrt{g}}\left(-g_{12} \phi_{\xi}+g_{22} \phi_{\eta}\right)\right]_{s}^{n}+S^{\phi} r \sqrt{g} \Delta \eta \Delta \xi
\end{aligned}
$$

where the convection coefficients:

$$
\begin{array}{ll}
\mathrm{C}_{\mathrm{e}}=\left(\rho \mathrm{U}_{\mathrm{c}} \Delta \eta\right)_{\mathrm{e}} & \mathrm{C}_{\mathrm{w}}=\left(\rho \mathrm{U}_{\mathrm{c}} \Delta \eta\right)_{\mathrm{w}} \\
\mathrm{C}_{\mathrm{n}}=\left(\rho \mathrm{U}_{\mathrm{c}} \Delta \eta\right)_{n} & \mathrm{C}_{\mathrm{s}}=\left(\rho \mathrm{U}_{\mathrm{c}} \Delta \eta\right)_{\mathrm{s}}
\end{array}
$$

are obtained by linear interpolation within the physical plane, and the interpolation procedure is given by Rhie (1981). The source term, $S^{\Phi}$, is conveniently written as:

$$
S^{\phi}=S_{u}^{\phi}+S_{p}^{\phi} \phi
$$

Equation 5.10 is now written in the following compact notation:

$$
F_{e}-F_{w}+F_{n}-F_{s}=S_{u}^{\phi}+S_{p}^{\phi} \phi_{p}-E_{e}^{\varphi}\left(\phi_{\eta}\right)_{e}+E_{w}^{\phi}\left(\phi_{\eta}\right)_{w}-E_{\eta}^{\phi}\left(\phi_{\xi}\right)_{n}+E_{s}^{\phi}\left(\phi_{\xi}\right)_{s}
$$


where:

$$
\begin{aligned}
& F_{e}=D_{e}^{\phi}\left(\phi_{p}-\phi_{E}\right)+C_{e}^{\phi} \phi_{e} \\
& F_{w}=D_{w}^{\phi}\left(\phi_{P}-\phi_{w}\right)+C_{w}^{\phi} \phi_{w} \\
& F_{n}=D_{n}^{\phi}\left(\phi_{p}-\phi_{N}\right)+C_{n}^{\phi} \phi_{n} \\
& F_{s}=D_{s}^{\phi}\left(\phi_{P}-\phi_{S}\right)+C_{s}^{\phi} \phi_{s} \\
& D_{e}^{\phi}=\left(\frac{\Gamma^{\phi}}{\sqrt{g}} g_{22} \frac{r \Delta \eta}{\delta \xi}\right)_{e} \\
& D_{w}^{\phi}=\left(\frac{\Gamma^{\phi}}{\sqrt{g}} g_{22} \frac{r \Delta \eta}{\delta \xi}\right)_{w} \\
& D_{n}^{\phi}=\left(\frac{\Gamma^{\phi}}{\sqrt{g}} g_{11} \frac{r \Delta \xi}{\delta \eta}\right)_{n} \\
& D_{s}^{\phi}=\left(\frac{\Gamma^{\phi}}{\sqrt{g}} g_{11} \frac{r \Delta \xi}{\delta \eta}\right)_{s} \\
& E_{e}^{\phi}=\left(\frac{\Gamma}{\sqrt{g}} g_{12} \frac{r \Delta \eta}{\delta \eta}\right)_{e} \\
& E_{w}^{\phi}=\left(\frac{\Gamma}{\sqrt{g}} g_{12} \frac{r \Delta \eta}{\delta \eta}\right)_{w} \\
& E_{n}^{\phi}=\left(\frac{\Gamma^{\phi}}{\sqrt{g}} g_{12} \frac{r \Delta \xi}{\delta \xi}\right)_{n} \\
& E_{s}^{\phi}=\left(\frac{\Gamma}{\sqrt{g}} g_{12} \frac{r \Delta \xi}{\delta \xi}\right)_{s} .
\end{aligned}
$$

By properly finding the convective cell-face variables $\left(\phi_{\mathrm{e}}, \phi_{\mathrm{w}}, \phi_{\mathrm{n}}, \phi_{\mathrm{s}}\right)$, Equation $5.11 \mathrm{can}$ be written as:

$$
\begin{array}{r}
a_{p}^{\phi} \phi_{p}=a_{e}^{\phi} \phi_{E}+a_{w}^{\phi} \phi_{w}+a_{n}^{\phi} \phi_{N}+a_{s}^{\phi} \phi_{s}+S_{u}^{\phi} \sqrt{g} r \Delta \xi \Delta \eta \\
-E_{e}^{\phi}\left(\phi_{\eta}\right)_{e}+E_{w}^{\phi}\left(\phi_{\eta}\right)_{w}-E_{n}^{\phi}\left(\phi_{\xi}\right)_{n}+E_{s}^{\phi}\left(\phi_{\xi}\right)_{s}
\end{array}
$$

where:

$$
a_{p}^{\phi}=a_{e}^{\phi}+a_{w}^{\phi}+a_{n}^{\phi}+a_{s}^{\phi}-S_{p}^{\phi}
$$

Notice that the truncation errors in the diffusion terms are third order for uniform grids (second-order central differencing of the second derivatives), while the truncation errors in the 
convective terms are dependent upon the interpolation method for the cell-face variables. The use of schemes like first-order upwind approximation for the convective terms would introduce serious false-numerical diffusion errors. Consequently, the convective terms are modified to eliminate the numerical diffusion by various differencing approximations.

\subsubsection{Differencing of the Convection Terms}

In a series of papers, Leonard (1988, 1991), Gaskell and Lau (1988), and Leonard and Mokhtari (1990) discuss the state of the art of modeling convective terms. The convective-term modeling has been an embarrassment for the numerical heat- and mass-transfer community because the convective terms are modeled by the first-order upwinding in the form of hybrid scheme of Spalding (1972) and the power-law scheme of Patankar (1980). However, the approach selected by the aerodynamics community is far superior.

Leonard (1991) summarizes the following three distinct problems associated with the convective-term modeling: (1) numerical diffusion associated with the first-order upwinding, (2) unphysical oscillations inherent in the second-order methods, and (3) unacceptable global artificial diffusion in schemes that switch between the first-order upwinding and second-order methods. Considerable progress has been made to cure these problems.

The approaches to derive high-resolution, oscillation-free convective-differencing schemes can be summarized as follows: (1) add a limited antidiffusive flux to the first-order upwind scheme (employed by aerodynamists), (2) add a smoothing-diffusive flux to the unbounded and/or higher-order schemes to damp the oscillations, and (3) derive a composite procedure with different schemes, provided that there is an appropriate switch between them under a specified criterion. It is quite obvious that there is always a trade-off between the accuracy and boundness because the former requires the diminishing numerical diffusion, while the latter requires an increasing diffusion.

The normalized variable in terms of the upwind biased node values is defined as:

$$
\Phi=\frac{\phi-\phi_{U}}{\phi_{D}-\phi_{U}}
$$

where $\mathrm{U}, \mathrm{C}$, and D denote upstream, center, and downstream nodes, respectively. The upwind nodes for a given control-volume face are chosen according to the control-volume-face velocity. Some of the popular convective-discretization schemes in upwind-biased normalized form are shown in Table 5.1 .

The accuracy and boundness of the discretization schemes can be determined with a normalized variable diagram, as shown in Figure 5.2. The range $0 \leq \Phi_{C} \leq 1$ is called the 
TABLE 5.1 Some Well-Known Discretization Schemes ${ }^{a}$

\begin{tabular}{lll}
\hline Scheme & Normalized & Nonnormalized \\
\hline
\end{tabular}

First-order upwind (UD)

$$
\Phi_{\mathrm{f}}=\Phi_{\mathrm{C}} \quad \phi_{\mathrm{f}}=\phi_{\mathrm{C}}
$$

Central $(C D)$

$$
\Phi_{f}=\frac{3}{4}+\frac{1}{2}\left(\Phi_{C}-\frac{1}{2}\right) \quad \phi_{f}=\frac{\phi_{C}+\phi_{D}}{2}
$$

Second-order upwind (SUD) $\quad \Phi_{\mathrm{f}}=\frac{3}{2} \Phi_{\mathrm{C}} \quad \phi_{\mathrm{f}}=\frac{\phi_{\mathrm{C}}+\phi_{\mathrm{D}}}{2}-\frac{1}{2}\left(\phi_{\mathrm{D}}-2 \phi_{\mathrm{C}}+\phi_{\mathrm{U}}\right)$

Qulck

$$
\Phi_{\mathrm{f}}=\frac{3}{8}+\frac{3}{4} \Phi_{\mathrm{C}} \quad \phi_{\mathrm{f}}=\frac{\phi_{\mathrm{C}}+\phi_{\mathrm{D}}}{2}-\frac{1}{8}\left(\phi_{\mathrm{D}}-2 \phi_{\mathrm{C}}+\phi_{\mathrm{U}}\right)
$$

$a_{\Phi}=$ normalized variable; $\phi=$ arbitrary variable; $f=$ cell face; $C=$ center node;

$D=$ downstream node; and $U=$ upstream node.

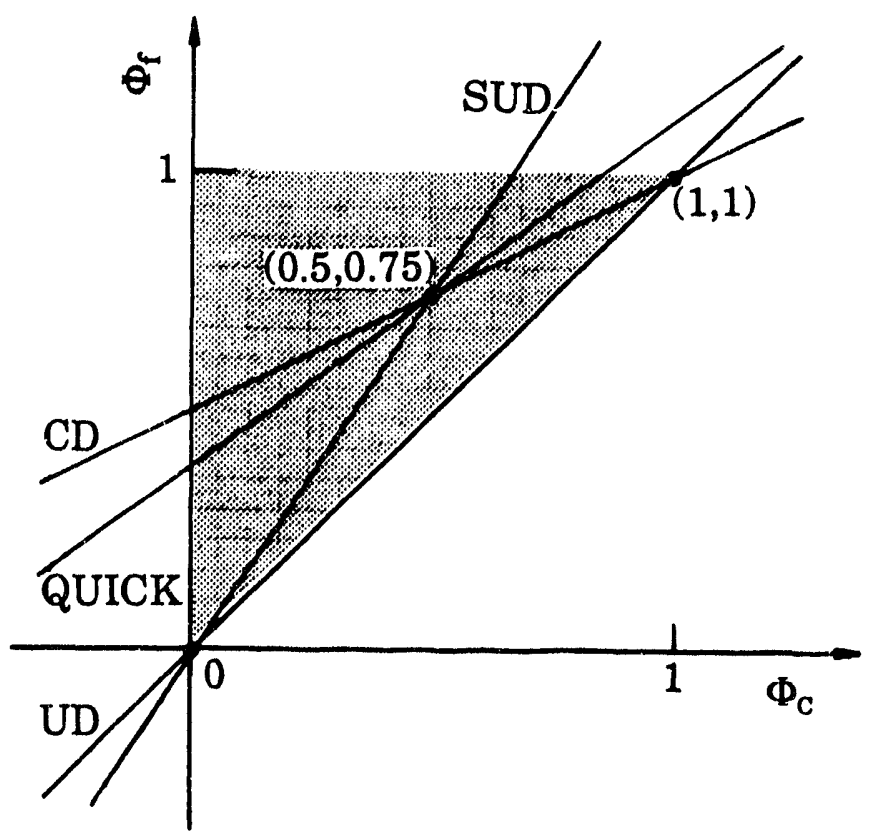

FIGURE 5.2 Normalized Variable Diagram 
monotonic range and usually accounts for the bulk of the flow field. The shaded upper triangle is the bounded region that satisfies the convection boundness criterion (CBC) of Gaskell and Lau (1988). A numerical discretization in the monotonic range is bounded if:

1. $\Phi_{\mathrm{f}}=\mathrm{f}\left(\Phi_{\mathrm{C}}\right)$ curve passes through the point $(0.0)$ and (1.1),

2. and $\Phi_{\mathrm{f}} \leq 1$,

3. and $\Phi_{\mathrm{f}} \leq \Phi_{\mathrm{C}}$.

One can see that there is considerable flexibility for choice of the convective modeling, provided that the CBC criterion in the monotonic range is met and the derivative, $\partial \Phi_{\mathrm{f}} / \partial \Phi_{\mathrm{C}}$, remains positive. Further, Leonard and Mokhtari (1990) suggested that if the $\Phi_{\mathrm{f}}$ curve passes through the point $(0.5,0.75)$, the scheme is at least second-order accurate (e.g., central [CD], second-order upwind [SUD]). If the curve passes through the point $(0.5,0.75)$ with a slope of 0.75 , the scheme is then third-order accurate (e.g., QUICK). Otherwise, the scheme is first-order accurate (e.g., first-order upwind [UD]).

Figure 5.2 clearly shows that the first-order upwind scheme is the most diffusive and satisfies the CBC. The rest of the discretization schemes do not satisfy the CBC. Therefore, it can be concluded that the well-known engineering schemes do not unconditionally and simultaneously achieve monotonicity, high accuracy, and convective stability. To achieve these qualities, one has to employ a nonlinear scheme in the monotonic range. In addition to the hybrid (upwind/central) (Spalding 1972), the second-order upwind, and the QUICK schemes (Leonard 1979), three convective-discretization schemes - Zhu (1991), Gaskell and Lau (1988), and a new composite scheme - were added to the program to achieve both accuracy and stability .

The parabolic/upwind composite scheme (Zhu 1991) is a composite of parabolic and the first-order upwind schemes, where:

$$
\Phi_{\mathrm{f}} \Phi_{\mathrm{f}}= \begin{cases}\Phi_{\mathrm{C}}+\left(\Phi_{\mathrm{C}}-\Phi_{\mathrm{C}}{ }^{2}\right) & \text { if } 0<\Phi_{\mathrm{C}}<1 \\ \Phi_{\mathrm{C}} & \text { otherwise }\end{cases}
$$


The SMART scheme (Gaskell and Lau 1988) is a composite of QUICK and the first-order upwind schemes, where:

$$
\Phi_{\mathrm{f}}= \begin{cases}3 \Phi_{\mathrm{C}} & 0<\Phi_{\mathrm{C}}<\frac{1}{6} \\ \frac{3}{4} \Phi_{\mathrm{C}}+\frac{3}{8} & \frac{1}{6} \leq \Phi_{\mathrm{C}} \leq \frac{5}{6} \\ 1 & \frac{5}{6}<\Phi_{\mathrm{C}} \leq 1 \\ \Phi_{\mathrm{C}} & \text { otherwise }\end{cases}
$$

The new composite scheme is a composite of the central, parabolic, and the second-order upwind schemes, where:

$$
\Phi_{f}= \begin{cases}0.75+0.5\left(\Phi_{C}-0.5\right) & \text { if } 1<\Phi_{C} \\ \Phi_{C}+\left(\Phi_{C}-\tilde{\Phi}_{C}^{2}\right) & \text { if } 0 \leq \Phi_{C} \leq 1 \\ 1.5 \Phi_{C} & \text { if } \Phi_{C}<0\end{cases}
$$

As expected, it was found that the new composite scheme and the parabolic/upwind scheme of Zhu (1991) are very stable and nonoscillatory. However, the SMART scheme requires much more underrelaxation than the other two schemes. Figure 5.3 shows the normalized variable diagram of the new composite scheme, and it is at least second-order correct.

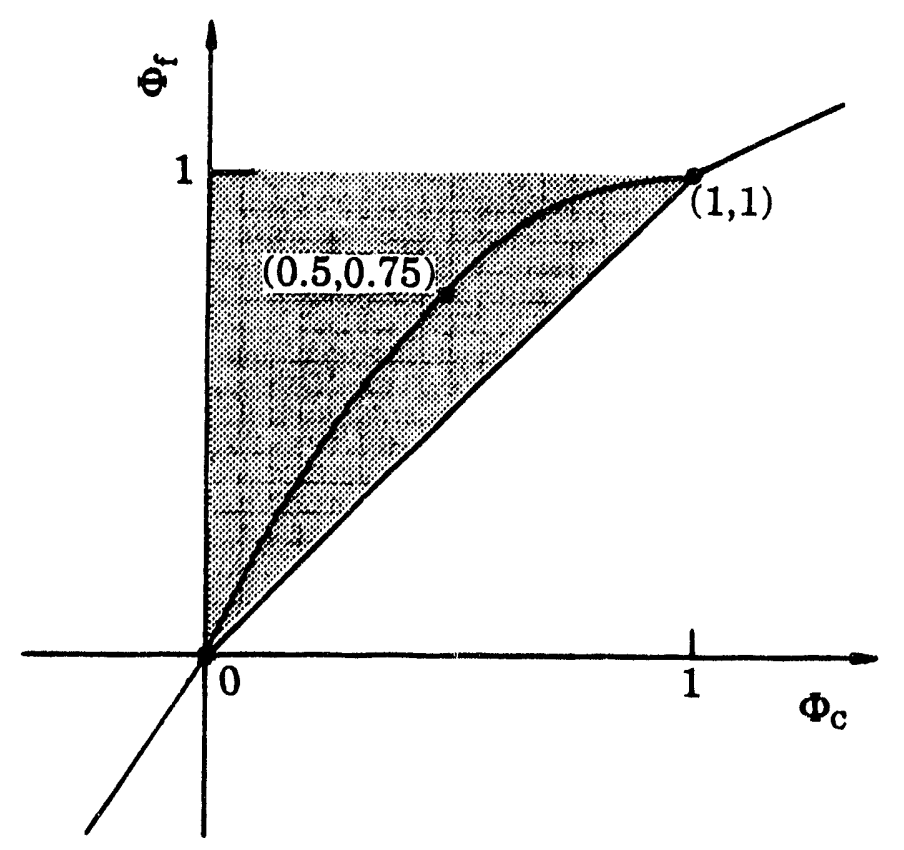

FIGURE 5.3 Normalized Variable Diagram for New Composite Scheme 


\subsubsection{Interpolation for Cell-Face Velocities}

The initial studies with the nonorthogonal curvilinear coordinates with a nonstaggered grid used a linear interpolation to recalculate cell-face velocities (Rhie 1981; Rhie and Chow 1983). However, numerical oscillations and underrelaxation dependency of the convergence were experienced with the linear interpolation. Majumdar (1988) suggested a better interpolating method for the velocities by means of the momentum equations (called momentum interpolation). This method was subsequently called the pressure-weighted interpolation method (Miller and Schmidt 1988; Peric et al. 1988; Kobayashi and Pereira 1991).

The following is a derivation of the pressure-weighted interpolation method. The axial and radial momentum equations are written as:

$$
\begin{aligned}
& u_{P}=\frac{1}{a_{P}^{u}}\left(\sum_{N E W S} a_{n b}^{u} u_{n b}+S_{T}^{u}\right)_{P}+\left(b^{u} p_{\xi}+c^{u} p_{\eta}\right)_{P} \\
& v_{P}=\frac{1}{a_{p}^{v}}\left(\sum_{N E W S} a_{n b}^{v} v_{n b}+S_{T}^{v}\right)_{P}+\left(b^{v} p_{\xi}+c^{v} p_{\eta}\right)_{P}
\end{aligned}
$$

where $S_{\mathrm{T}}^{\mathrm{u}}$ and $\mathrm{S}_{\mathrm{T}}^{\mathrm{v}}$ are the residual source terms after the pressure terms are excluded and:

$$
\begin{array}{ll}
\mathrm{b}^{\mathrm{u}}=-\frac{\mathrm{ry}_{\eta} \Delta \xi \Delta \eta}{\mathrm{a}_{\mathrm{p}}^{\mathrm{u}}} & \mathrm{b}^{\mathrm{v}}=\frac{\mathrm{ry}_{\xi} \Delta \xi \Delta \eta}{\mathrm{ap}_{\mathrm{p}}^{\mathrm{u}}} \\
\mathrm{c}^{\mathrm{u}}=\frac{\mathrm{rx}_{\eta} \Delta \xi \Delta \eta}{\mathrm{a}_{\mathrm{p}}^{\mathrm{v}}} & \mathrm{c}^{\mathrm{v}}=-\frac{\mathrm{rx}_{\xi} \Delta \xi \Delta \eta}{\mathrm{a}_{\mathrm{p}}^{\mathrm{v}}} .
\end{array}
$$

Written in the short notation, Equations 5.13a and 5.13b become:

$$
\begin{aligned}
& u_{P}=H(u)_{P}+\left(b{ }^{u} p_{\xi}+c p_{\eta}\right)_{P} \\
& v_{P}=H(v)_{P}+\left(b{ }^{u} p_{\xi}+c{ }^{u} p_{\eta}\right)_{P} .
\end{aligned}
$$

Employing underrelaxation yields the following:

$$
u_{p}=H(u)_{p}+\left(1-\alpha^{u}\right) u_{p}^{*}+\left(B^{u} p_{\xi}+C^{u} p_{\eta}\right) p
$$




$$
v_{P}=H(v)_{P}+\left(1-\alpha^{v}\right) v_{P}^{*}+\left(B^{v} p_{\xi}+C^{v} p_{\eta}\right)_{P}
$$

where the starred velocities $u_{\mathrm{p}}^{*}$ and $v_{\mathrm{p}}^{*}$ represent the velocities from the previous iteration and

$$
\begin{array}{ll}
\mathrm{B}^{\mathrm{u}}=\alpha^{\mathrm{u}} \mathrm{b}^{\mathrm{u}} & \mathrm{C}^{\mathrm{u}}=\alpha^{\mathrm{u}} \mathrm{c}^{\mathrm{u}} \\
\mathrm{B}^{\mathrm{v}}=\alpha^{\mathrm{v}} \mathrm{b}^{\mathrm{v}} & \mathrm{C}^{\mathrm{v}}=\alpha^{\mathrm{v}} \mathrm{c}^{\mathrm{v}} .
\end{array}
$$

Equations $5.15 \mathrm{a}$ and $5.15 \mathrm{~b}$ are linearly interpolated to the cell faces to give the cell-face velocities:

$$
\begin{aligned}
& u_{e}=\overline{H\left(u_{e}\right)}+\left(1-\alpha^{u}\right) \vec{u}_{e}^{*}+\left(\overline{B^{u}} p_{\xi}+\overline{C^{u}} p_{\eta}\right)_{e} \\
& v_{e}=\overline{H\left(v_{e}\right)}+\left(1-\alpha^{v}\right) \vec{v}_{e}^{*}+\left(\overline{B^{v}} p_{\xi}+\overline{C^{v}} p_{\eta}\right)_{e} .
\end{aligned}
$$

Similarly, this procedure is used for the other three faces of the control volume.

\subsubsection{Pressure-Velocity Coupling}

The main difficulty in calculating the velocities lies in the unknown pressure field. The current program uses a pressure-correction equation, which is derived from the continuity equation to correct the pressure field and, subsequently, the new velocity fields. The following derivation for the pressure-correction equation is due to Patankar and Spalding (1972) and was first applied to the curvilinear coordinates by Rhie (1981) and Rhie and Chow (1983). Axial and radial momentum equations are written as:

$$
\begin{aligned}
& u_{P}^{*}=H\left(u^{*}\right)_{P}+\left(1-\alpha^{u}\right) u_{P}^{*}+\left(B^{u} p_{\xi}^{*}+C^{u} p_{\eta}^{*}\right)_{P} \\
& v_{P}^{*}=H\left(v^{*}\right)_{P}+\left(1-\alpha^{v}\right) v_{P}^{*}+\left(B^{v} p_{\xi}^{*}+C^{v} p_{\eta}^{*}\right)_{P}
\end{aligned}
$$

The starred velocities represent approximate velocity field values based on the estimated pressure field. The cell-face velocities are:

$$
u_{e}^{*}=\overline{H\left(u^{*}\right)_{e}}+\left(1-\alpha^{u}\right) u_{e}^{*}+\left(\overline{B^{u}} p_{\xi}^{*}+\overline{C^{u}} p_{\eta}^{*}\right) e
$$




$$
v_{e}^{*}=\overline{H\left(v^{*}\right)_{e}}+\left(1-\alpha^{v}\right) v_{e}^{*}+\left(\overline{B^{v}} p_{\xi}^{*}+\overline{C^{v}} p_{\eta}^{*}\right) e .
$$

The velocity corrections then can be derived by subtracting the guessed velocities given by Equations 5.17a, 5.17b, 5.18a, and 5.18b from the true velocities to get the following approximate relations:

$$
\begin{aligned}
& u_{p}=u_{p}^{*}+\left(B^{u} p^{\prime} \xi+C^{u} p^{\prime} \eta\right)_{p} \\
& v_{p}=v_{p}^{*}+\left(B^{v} p^{\prime} \xi+C^{v} p^{\prime} \eta\right)_{p} \\
& u_{e}=u_{e}^{*}+\left(\overline{B^{u}} p^{\prime} \xi+\overline{C^{u}} p^{\prime} \eta\right)_{e} \\
& v_{e}=v_{e}^{*}+\left(\overline{B^{v}} p^{\prime} \xi+\overline{C^{v}} p^{\prime} \eta\right)_{e} .
\end{aligned}
$$

Introducing these relations into the discretized continuity equation and rearranging them give an equation for the pressure corrections:

$$
a_{p}^{p^{\prime}} p_{p}^{\prime}=a_{e}^{p^{\prime}} p_{E}^{\prime}+a_{w}^{p^{\prime}} p_{w}^{\prime}+a_{n}^{p^{\prime}} p_{N}^{\prime}+a_{s}^{p^{\prime}} p_{S}^{\prime}+s^{p^{\prime}}
$$

where:

$$
\begin{aligned}
& a_{e}^{p^{\prime}}=-\rho\left(\overline{B^{u}} y_{\eta}-\overline{B^{v}} x_{\eta}\right)_{e}^{r \Delta \eta} \\
& a_{e}^{p^{\prime}}=-\rho\left(\overline{B^{u}} y_{\eta}-\overline{B^{v}} x_{\eta}\right)_{e}^{r \Delta \eta} \\
& a_{n}^{p^{\prime}}=-\rho\left(\overline{C^{v}} x_{\xi}-\overline{C^{u}} x_{\xi}\right)_{h} r \Delta \xi \\
& a_{s}^{p^{\prime}}=-\rho\left(\overline{C^{v}} x_{\xi}-\overline{C^{u}} x_{\xi}\right)_{s} r \Delta \xi \\
& a_{p}^{p^{\prime}}=a_{e}^{p^{\prime}}+a_{w}^{p^{\prime}}+a_{s}^{p^{\prime}}+a_{n}^{p^{\prime}} \\
& S^{p^{\prime}}=-\left(\rho U_{c}^{*} r \Delta \eta\right)_{w}^{e}-\left(\rho V_{c}^{*} r \Delta \xi\right)_{s}^{n}-\left[\rho\left(\overline{C^{u}} y_{\eta}-\overline{C^{v}} y_{\eta}\right) p^{\prime} r \Delta \eta\right]_{w}^{e} \\
& \quad-\left[\rho\left(\overline{B^{v}} x_{\xi}-\overline{B^{u}} y_{\xi}\right) p^{\prime} r \Delta \xi\right]_{s}^{n} .
\end{aligned}
$$


This Poisson equation for the pressure correction has the same coefficient structure as Equation 5.12; therefore, it can be solved by using the same matrix inversion technique. The pressures corrected as:

$$
p=p^{*}+\alpha^{p} p^{\prime}
$$

where $\mathrm{p}^{*}$ denotes the pressures from previous iteration.

\subsubsection{Solution Procedure}

The discretized algebraic equations (Equations 5.11, 5.17a, and 5.17b) are solved by employing a line-by-line tridiagonal matrix algorithm, often referred to as the line-by-line Thomas algorithm described by Patankar (1980). Since the results are not exact solutions of the algebraic equations, a sweeping procedure is applied until the necessary convergence criterion is achieved. The sweeping in one direction may not be adequate to reach this convergence criterion; therefore, an option of sweeping in both directions is included. Further, the number of sweeps in each direction is increased to decrease the error from iteration to iteration. A residual mass-reduction criterion is also implemented to stop the sweeping action when a given inner convergence criterion is achieved.

The discretized algebraic equations are solved by using the above-noted line-by-line Thomas algorithm in a segregated manner by employing a SIMPLEC (SIMPLE-Consistent) procedure (Van Doormaal and Raithby 1984). This procedure is a variant of the well-known SIMPLE solution procedure (Patankar and Spalding 1972).

The solution procedure involves the following major steps:

1. Guess the pressure field, $p^{*}$.

2. Solve Equations $5.15 \mathrm{a}$ and $5.15 \mathrm{~b}$ for the starred velocities, $\mathrm{u}^{*}$ and $\mathrm{v}^{*}$.

3. Calculate cell-face velocities $u_{c}^{*}$ and $v_{c}^{*}$ with Equations $5.18 \mathrm{a}$ and $5.18 \mathrm{~b}$ and calculate $\mathrm{U}_{\mathrm{c}}^{*}$ and $\mathrm{V}_{\mathrm{c}}^{*}$ with Equations 5.5a and 5.5b.

4. Solve Equation 5.20 for the pressure corrections and correct the pressure field with Equation 5.22.

5. Correct the velocities $U_{c}$ and $V_{c}$ by using Equations $5.19 c$ and $5.19 d$ and store these values at the cell faces. 
6. Correct the physical velocities ( $u$ and $v$ ) by using Equations $5.19 \mathrm{a}$ and $5.19 \mathrm{~b}$.

7. Solve Equation 5.11 for the scalars, $T, k$, and $\varepsilon$.

8. Repeat step 2 if the convergence criterion is not met.

In addition to the SIMPLE and SIMPLEC solution algorithms, the program also contains the PISO algorithm (Pressure-Implicit Solution Operator) (Issa et al. 1986).

\subsection{Convergence Criteria}

During the numerical studies, extreme care was given to the numerical-diffusion issue, the grid dependency, and the convergence. The numerical diffusion-related issues were discussed in Section 5.3. To minimize numerical diffusion, both the orthogonal and body-fitted programs employed at least second-order finite-differencing for the convective-term discretization during the final runs. Specifically, the orthogonal numerical code used the QUICK differencing scheme (Phillips and Schmidt 1985a), while the body-fitted code used the parabolic/upwind composite scheme of Zhu (1991). For the sake of brevity, only the body-fitted numerical code was discussed with regard to the grid dependency and convergence; however, the same care was given to the orthogonal-code simulations.

\subsubsection{Grid Dependency}

A requirement of turbulence modeling is that the first radial, near-wall grid point is located well within the laminar sublayer (i.e., $y^{+} \approx 0.1$ ). The grid in the radial direction expanded by a factor of about 1.05-1.2. The use of a very fine mesh in radial direction placed restrictions on the mesh in the axial direction. In particular, essentially the same mesh fineness was required to keep the aspect ratio from becoming too low. The typical mesh network was 150 in the axial direction and 50 in the radial direction for an enhanced tube geometry of $e / D=0.02, p / e=20$ and at Reynolds number of 10,000 . The grid dependence by employing different grid networks was tested.

\subsubsection{Convergence}

The program uses an iterative approach to obtain the final steady-state solution. The following factors affect the predicted results and the convergence. 


\subsubsection{Underrelaxation}

The program used underrelaxation as a tool to control the stability of the simulations. The colocated mesh network is especially prone to numerical oscillations and instability because of the interpolation of the cell-phase velocities. As a result, it requires heavy underrelaxation: 0.5 for both the axial and radial velocities and 0.5 for the pressure iterations. However, the underrelaxation factors sometimes were dropped to 0.3 for both the velocity and pressure iterations to prevent divergence.

\subsubsection{Inner Iterations}

During each global iteration, the equations were solved in a sequential manner with the line-by-line tridiagonal matrix algorithm. The line-by-line sweeps were performed, first in the radial direction and then in the axial direction. There was a preset limit to the number of the double sweeps performed, as well as a residual convergence criteria to stop. The preset limits were 5, 5, 10 , and 5 for $u, v$, pressure correction, and other scalar equations, respectively.

\subsubsection{Global Iterations}

The maximum number of global iterations and the global-residual stopping criteria determine the final convergence criteria of the numerical simulation. The iteration dependency tests were performed, and the maximum number of iterations were set accordingly. The global iterations were continued until the normalized mass residual (total mass residual divided by the mass flow rate) fell below $10^{-5}$ for the flow-field iterations. The global convergence criterion for the temperature field was also $10^{-5}$ for the normalized energy residual (the total heat balance residual divided by the total heat input). The maximum number of global iterations was 20,000 for the flow-field calculations and 30,000 for the temperature-field calculations. The typical run times were from 6 to more than $24 \mathrm{~h}$ on an IBM RS/6000-320 workstation. 


\section{Validation of the Numerical Programs}

This section of the report presents the validation of both the orthogonal and body-fitted numerical programs.

\subsection{Orthogonal Numerical Code}

The extensive database of Webb et al. (1971) is used for the validation of the frictionfactor and mean heat-transfer-coefficient predictions. The local heat-transfer data of Baughn and Roby (1992) are then used to show that the predicted local values are also in agreement with the experimental data. For these numerical runs, QUICK convective-differencing scheme and the twolayer turbulence model of Chen and Patel (1988) are employed.

\subsubsection{Comparison of Friction Factors}

Figure 6.1 compares the measured friction factors obtained by Webb et al. (1971) with the numerical predictions for the five tubes with the geometry listed in Table 2.2. Note the good agreement for the entire Reynolds number range considered and for all of the tubes.

\subsubsection{Comparisons of Mean Heat-Transfer Coefficient}

Figures 6.2 through 6.6 present comparisons of the measured and predicted mean Nusselt numbers as a function of the Reynolds number with the Prandtl number as a parameter for the $01 / 10,02 / 10,04 / 10,02 / 20$, and $02 / 40$ tubes used by Webb et al. (1971), respectively. Note the very favorable agreement.

Table 6.1 presents a comparison of the measured data obtained by Webb et al. (1971) and the numerical predictions for these five tubes and for the Prandtl numbers considered in their research. Note the good agreement, with an average error of only $15 \%$. In general, the numerical method underpredicts the experimental results with maximum errors occurring at the high Re range but never exceeding about $31 \%$.

\subsubsection{Comparisons of Local Heat-Transfer Coefflcient}

Further validation of the numerical model is possible through the use of the local heattransfer data of Baughn and Roby (1992). Figure 6.7 compares these experimental results with numerical predictions with air as the test fluid $(\operatorname{Pr}=0.71)$. Note the good agreement between the 


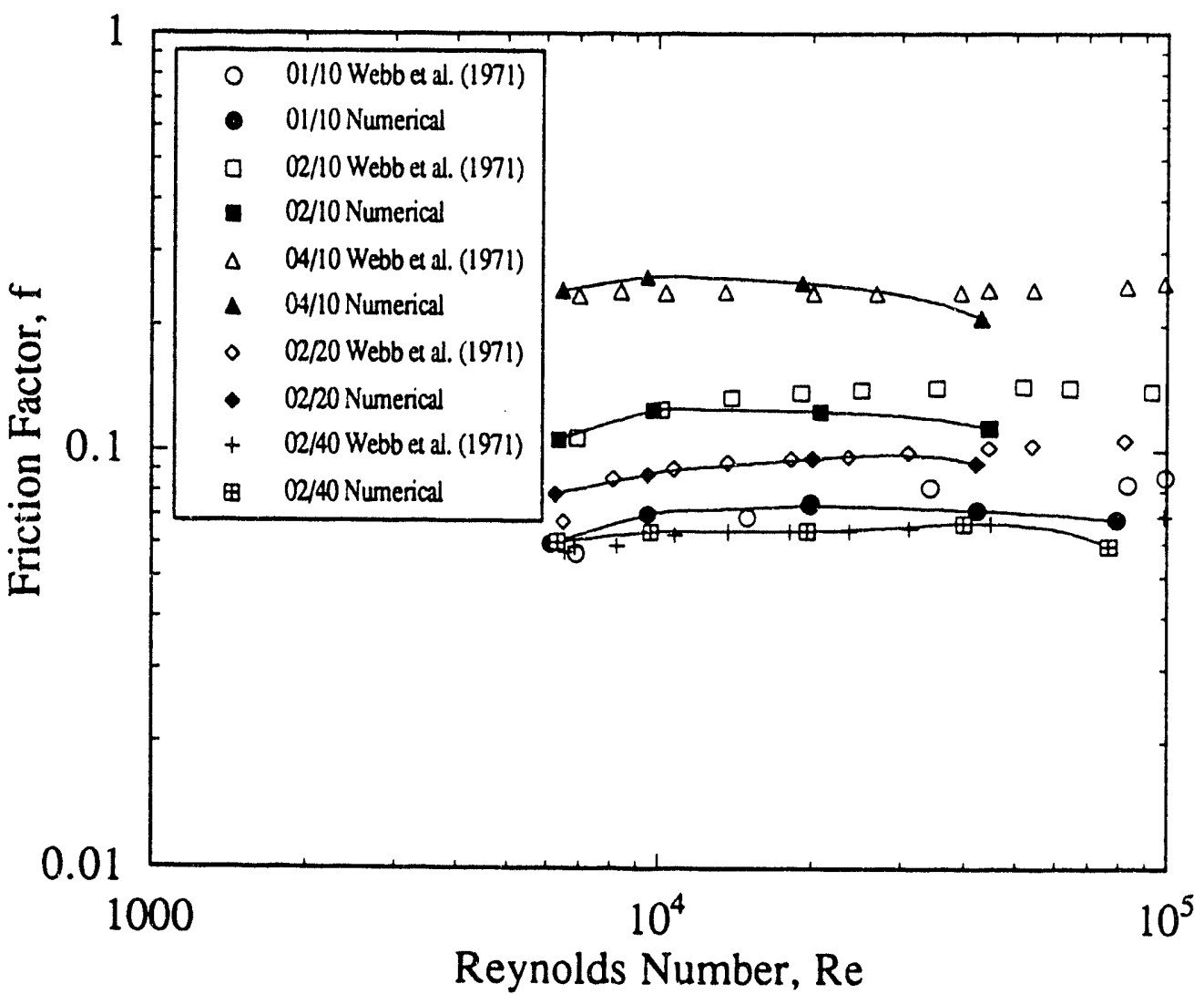

FIGURE 6.1 Comparison of Measured (Webb et al. 1971) and Predicted Friction Factors as a Function of the Reynolds Number

experimental and predicted results. These and other experimental results of Baughn and Yan (1992), Baughn and Yan (1991), and Berger and Hau, (1979) have demonstrated that the most significant contributions to the heat-transfer performance with the separation and reattachment mechanism occur at the top surface of the rib and in the downstream recirculation zone. These two maximums are clearly displayed in Figure 6.7.

\subsection{Body-Fitted Numerical Code}

Similar to the orthogonal code, the new curvilinear coordinate numerical code was first validated by comparisons with the friction-factor and heat-transfer values for a plain tube. Predicted friction-factor and heat-transfer values obtained with the new computer code are then compared with experimental results for some selected enhanced tube geometries. For these numerical runs, convective differencing scheme of Zhu (1991) and the two-layer turbulence model of Chen and Patel (1988) are employed. 


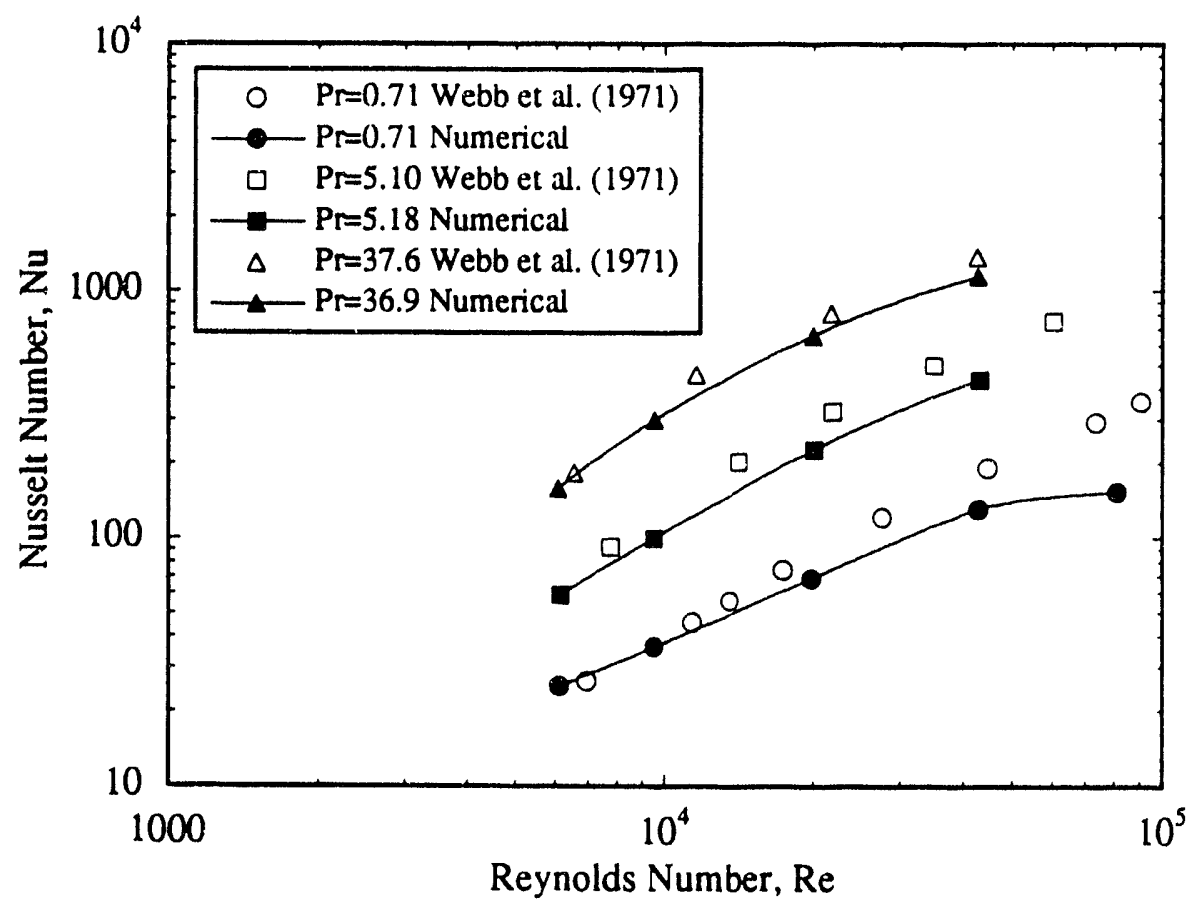

FIGURE 6.2 Comparison of Measured (Webb et al. 1971) and Predicted Mean Nusselt Numbers as a Function of the Roynolds Number with the Prandtl Number as a Parameter for the 01/10 Tube (Table 2.2)

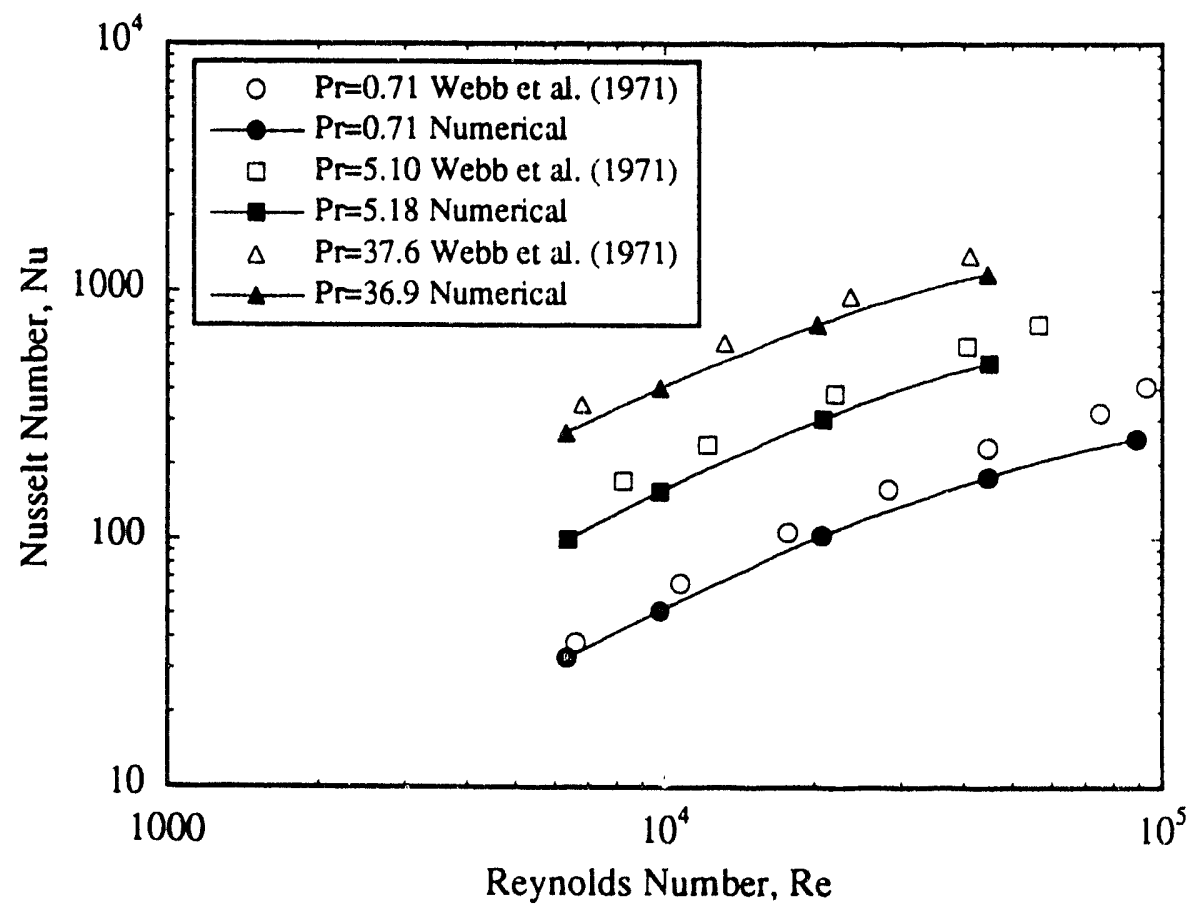

FIGURE 6.3 Comparison of Measured (Webb et al. 1971) and Predicted Mean Nusselt Numbers as a Function of the Reynolds Number with the Prandtl Number as a Parameter for the 02/10 Tube (Table 2.2) 


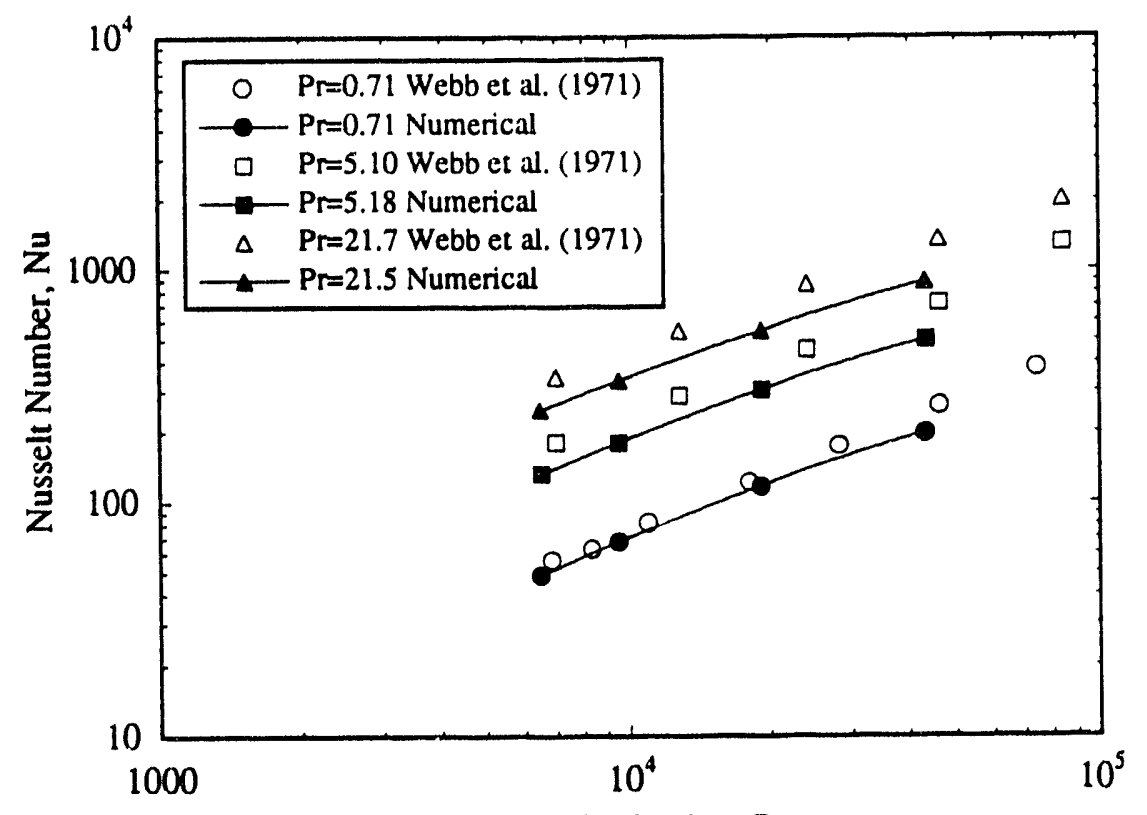

Reynolds Number, Re

FIGURE 6.4 Comparison of Measured (Webb et al. 1971) and Predicted Mean Nusselt Numbers as a Function of the Reynolds Number with the Prandtl Number as a Parameter for the 04/10 Tube (Table 2.2)

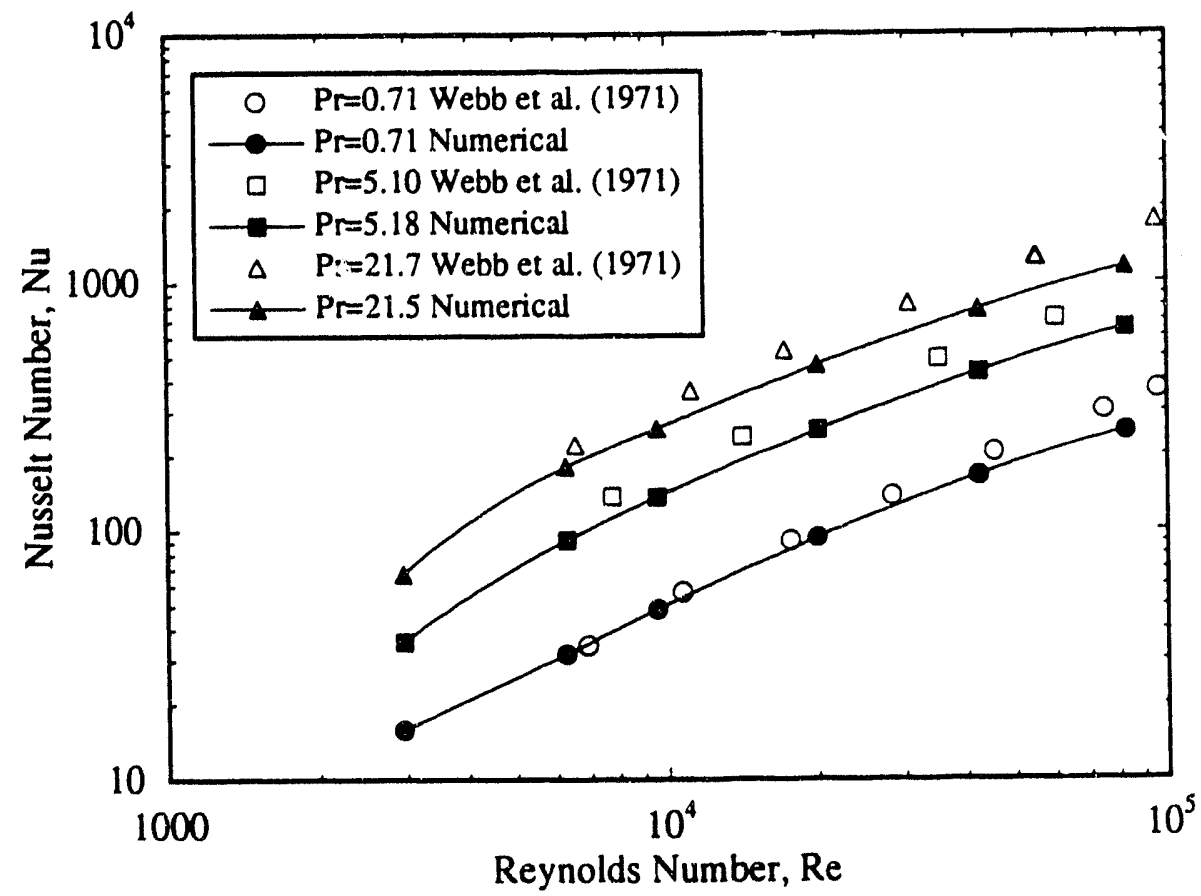

FIGURE 6.5 Comparison of Measured (Webb et al. 1971) and Predicted Mean Nusselt Numbers as a Function of the Reynolds Number with the Prandtl Number as a Parameter for the 02/20 Tube (Table 2.2) 


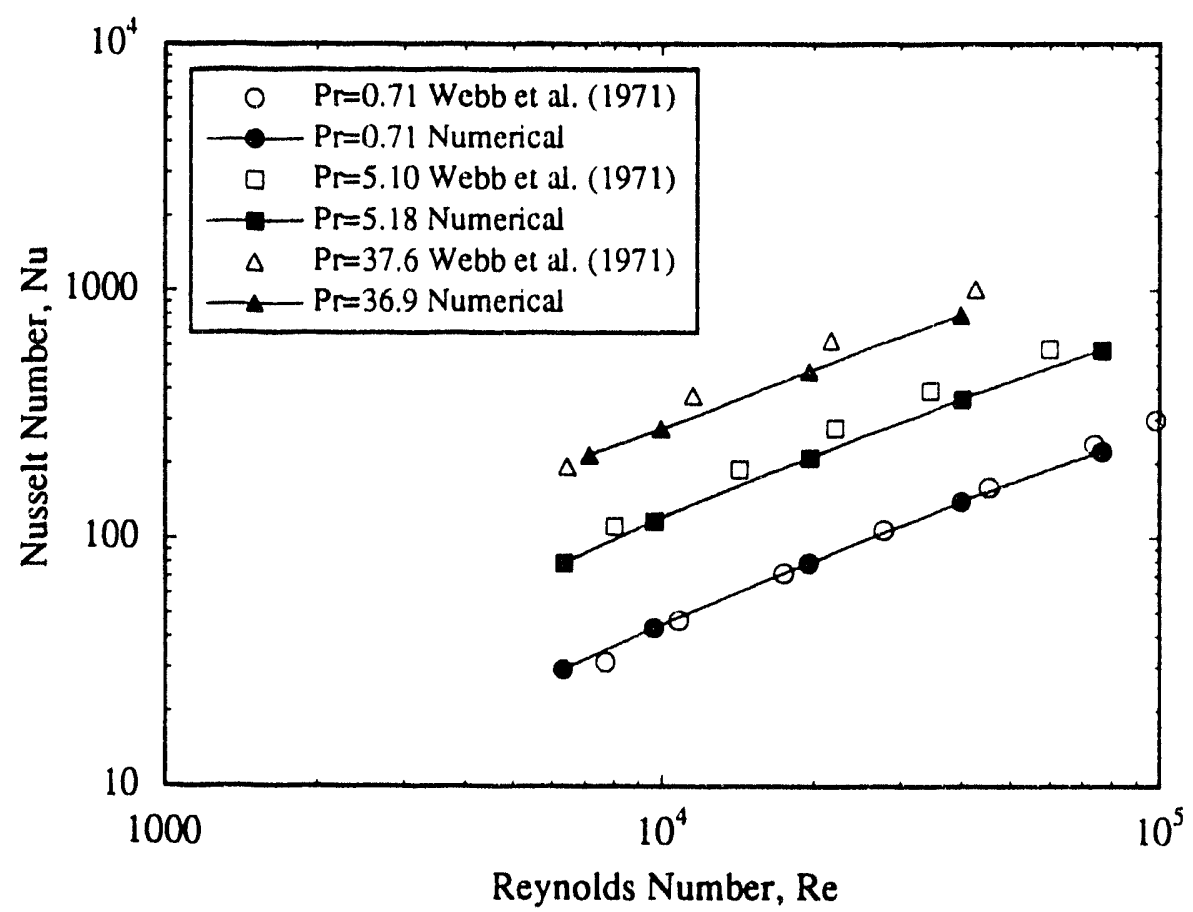

FIGURE 6.6 Comparison of Measured (Webb ot al. 1971) and Predicted Mean Nusselt Numbers as a Function of the Reynolds Number with the Prandil Number as a Parameter for the 02/40 Tube (Table 2.2)

Figure 6.8 shows that an excellent agreement is obtained between the predictions and the smooth-tube friction-factor correlation of Techo et al. (1965) and the Stanton number prediction method of Petukhov and Popov (Petukhov 1970). The Techo et al. (1965) friction-factor correlation was selected because it is an explicit form of the classical correlation called PKN (Prandtl-Kármán-Nikuradse) (Kakaç et al. 1987). With extensive experimental measurements, the PKN predictions agree within $\pm 2 \%$. The friction factors and the heat-transfer coefficients were ov spredicted by about $1.2 \%$ and $3.0 \%$, respectively.

A comparison of the measured and predicted friction-factor and heat-transfer values for an arc-shaped disruption is presented next. The experimental data were obtained by Hijikata and Mori (1987); the test fluid was water. Two tube geometries were selected for this comparison: (1) $\mathrm{e} / \mathrm{D}=0.02$ and $p / \mathrm{e}=44.4$ and $(2) \mathrm{e} / \mathrm{D}=0.04$ and $p / \mathrm{e}=22.2$. A width-to-height ratio of three, $w / e=3.0$, was selected for both tube geometries, although the exact value was not specified in the paper. Figure 6.9 shows that the numerical friction factors are overpredicted with a maximum deviation of about $26 \%$. Figure 6.10 shows that the Nusselt numbers are underpredicted with a maximum deviation of about $17 \%$.

A similar comparison was made with the data obtained by Nunner (1956) for semicircular transverse ribs with $\mathrm{e} / \mathrm{D}=0.04$ and $p / \mathrm{e}=20$. The test fluid for these experiments was air. Figures 6.11 and 6.12 show that satisfactory agreement is obtained between the numerically predicted and experimental values. 
TABLE 6.1 Comparison of Measured and Predicted Nusselt Numbers for the Five Tubes Tested by Webb et al. (1971)

\begin{tabular}{|c|c|c|c|c|c|c|c|c|}
\hline \multirow[b]{3}{*}{$\begin{array}{l}\text { Tube } \\
\text { Number }\end{array}$} & \multicolumn{8}{|c|}{ Prandtl Number } \\
\hline & \multicolumn{2}{|c|}{0.71} & \multicolumn{2}{|c|}{5.1} & \multicolumn{2}{|c|}{21.7} & \multicolumn{2}{|c|}{37.6} \\
\hline & Re & $\begin{array}{c}\% \\
\text { Deviationa }\end{array}$ & Re & $\begin{array}{c}\% \\
\text { Deviation }\end{array}$ & Re & $\begin{array}{c}\% \\
\text { Deviation }\end{array}$ & Re & $\begin{array}{c}\% \\
\text { Deviation }\end{array}$ \\
\hline \multirow{5}{*}{$01 / 10$} & & & & . & & & & \\
\hline & 6113 & -13.71 & 6142 & 12.08 & 6122 & 12.36 & 6080 & 3.57 \\
\hline & 9512 & 4.03 & 9506 & 18.28 & 9545 & 16.35 & 9537 & 9.77 \\
\hline & 19860 & 19.66 & 20054 & 22.53 & 20021 & 21.48 & 200145 & 16.97 \\
\hline & 42717 & 28.23 & 42905 & 27.10 & 42833 & 27.52 & 42684 & 15.40 \\
\hline \multirow[t]{4}{*}{$02 / 10$} & 6345 & 11.96 & 6383 & 27.10 & 6363 & 21.85 & 6321 & 17.55 \\
\hline & 9761 & 13.83 & 9750 & 22.16 & 9789 & 18.60 & 9782 & 15.62 \\
\hline & 20745 & 16.33 & 20833 & 17.58 & 20799 & 17.97 & 20316 & 14.65 \\
\hline & 44645 & 24.93 & 44847 & 19.47 & 44645 & 24.24 & 44610 & 19.76 \\
\hline \multirow[t]{4}{*}{$04 / 10$} & 6465 & 7.51 & 6489 & 21.22 & 6469 & 23.86 & & \\
\hline & 9497 & 6.34 & 9494 & 19.76 & 9533 & 23.45 & & \\
\hline & 19156 & 8.84 & 19238 & 19.42 & 19207 & 25.17 & & \\
\hline & 43157 & 18.94 & 43360 & 25.61 & 43282 & 31.23 & & \\
\hline \multirow[t]{5}{*}{$02 / 20$} & 6251 & -0.72 & 6287 & 18.70 & 6268 & 14.92 & & \\
\hline & 9540 & 1.80 & 9530 & 18.32 & 9567 & 18.74 & & \\
\hline & 19988 & 7.91 & 20067 & 20.09 & 20037 & 22.45 & & \\
\hline & 42131 & 15.19 & 42313 & 22.71 & 42243 & 26.02 & & \\
\hline & 82429 & 24.19 & 82436 & 26.02 & 82476 & 29.35 & & \\
\hline \multirow[t]{5}{*}{$02 / 40$} & 6319 & -13.73 & 6356 & 9.78 & 6336 & 4.61 & 7128 & 1.45 \\
\hline & 9665 & -6.93 & 9655 & 11.65 & 9691 & 11.02 & 9968 & 13.17 \\
\hline & 19599 & 0.68 & 19679 & 16.14 & 19649 & 19.19 & 19644 & 20.64 \\
\hline & 39928 & 3.87 & 40093 & 16.95 & 40030 & 22.28 & 39899 & 18.04 \\
\hline & 76605 & 8.67 & & & 76656 & 23.03 & & \\
\hline
\end{tabular}

a Average \% deviation $=15.35$. 


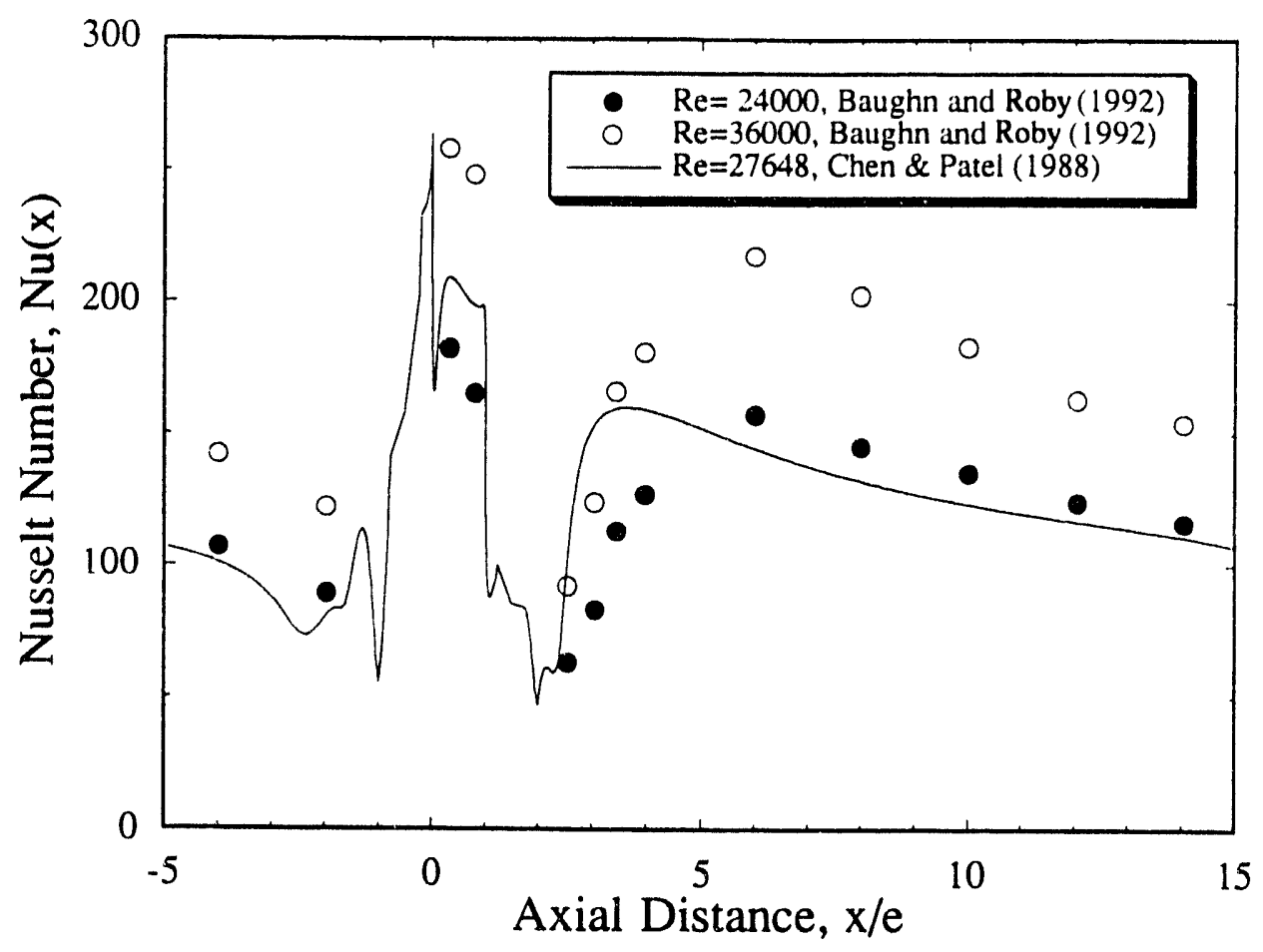

FIGURE 6.7 Comparison of Measured (Baughn and Roby 1992) and Predicted Local Nusselt Numbers as a Function of the Axial Location

These comparisons with experimental data clearly demonstrate that both the orthogonal and body-fitted numerical codes yield adequate predictions of the thermal-hydraulic performance. The next step is to use these codes to obtain an understanding of how the Prandtl number and the shape influence the performance of tubes with the separation and reattachment mechanism. 


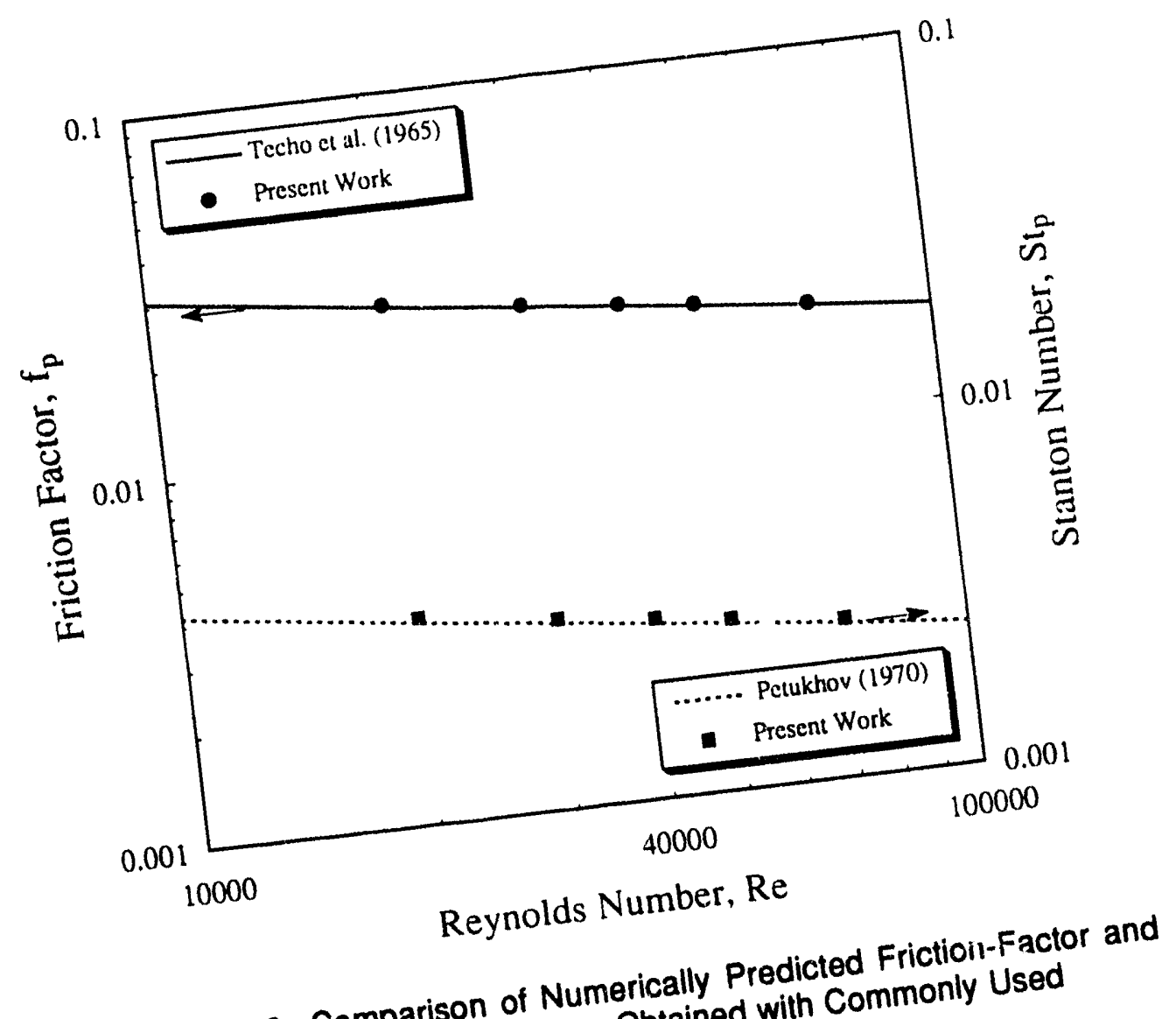

FIGURE 6.8 Comparison of Numerically Predicted Frommonly Used Stanton Number
Correlations

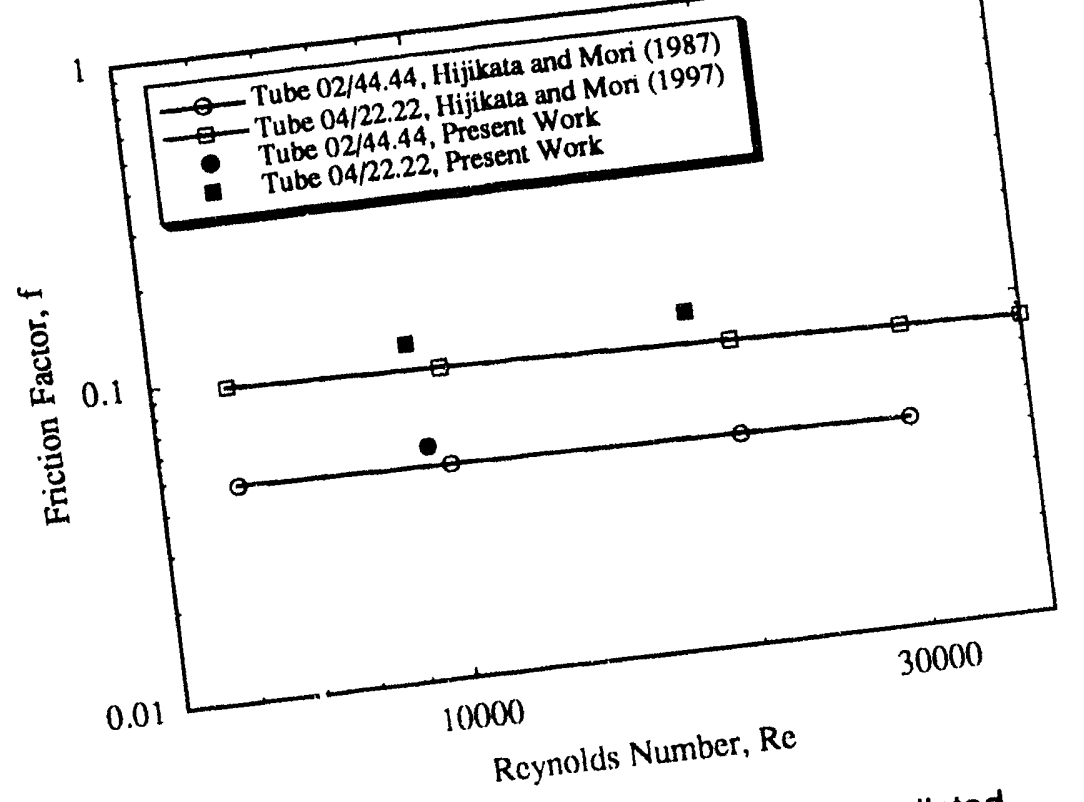

FIGURE 6.9 Comparison of the Nurierically Predicted Friction Factor with the Measured Arc-Shaped Disruption Hijikata and Mori (1987) 


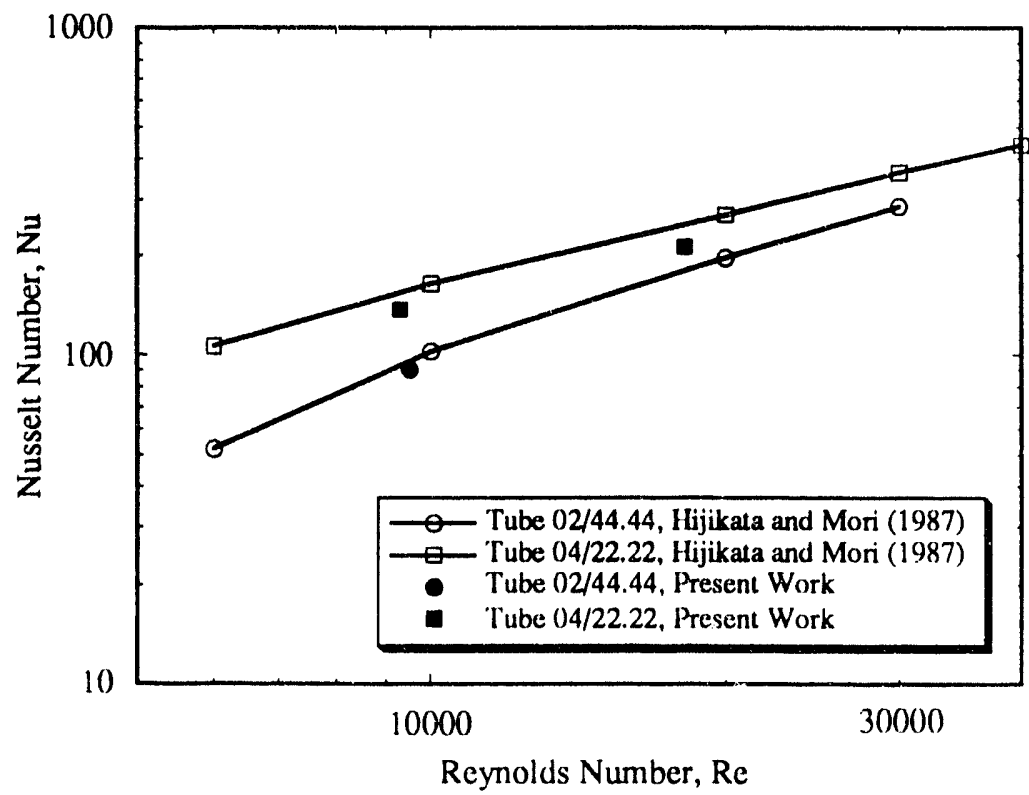

FIGURE 6.10 Comparison of the Numerically Predicted Nusselt Number with the Measured Values Obtained by Hijikata and Mori (1987) for an Arc-Shaped Disruption

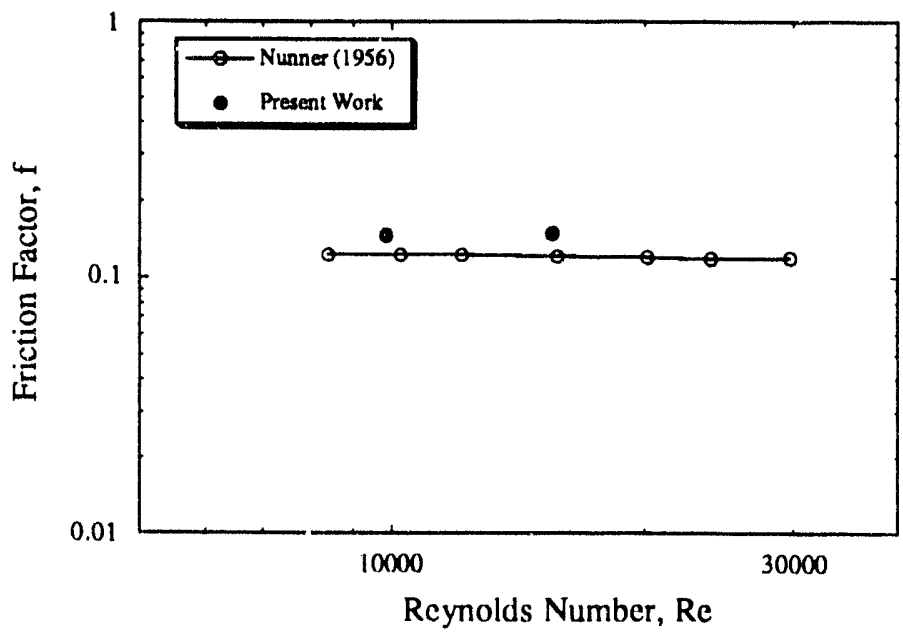

FIGURE 6.11 Comparison of the Numerically Predicted Friction Factor with the Measured Values Obtained by Nunner (1956) for a Semicircular Disruption 


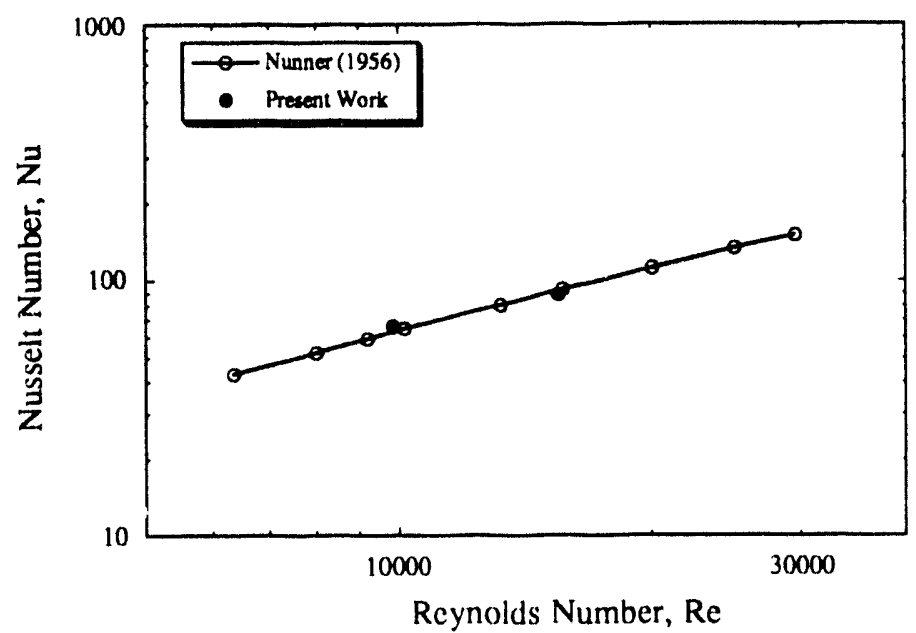

FIGURE 6.12 Comparison of the Numerically Predicted Nusselt Number with the Measured Values Obtained by Nunner (1956) for a Semicircular Disruption 


\section{Results and Discussion}

Section 6 demonstrated that the orthogonal and the body-fitted numerical codes are capable of predicting the thermal-hydraulic performance of enhanced tubes with the separation and reattachment enhancement mechanism. The orthogonal code is now used to explain the influence of the Prandtl number on the thermal performance, whereas the body-fitted code is used to determine the impact of different disruption shapes on the friction-factor and heat-transfer performance. The geometry types of the enhanced tubes selected for both investigations consist of transverse disruptions with spacings exceeding the reattachment length (i.e., the separation and reattachment enhancement mechanism).

\subsection{Influence of the Prandtl Number}

This section of the report will explain how the Prandtl number influences the enhancementlevel data in the manner shown in Figures 2.10 and 2.13. The tube geometries of Webb et al. (1971), shown in Table 2.2, will be used to support this explanation. The orthogonal numerical code is used for the following runs. These final runs are performed with QUICK differencing scheme and the two-layer model of Chen and Patel (1988).

An important concept toward an understanding of this approach is that six distinct regions, or zones, must be considered:

- Zone 1: upstream recirculation region;

- Zones 2, 3, and 4: the rib upstream, top, and downstream faces;

- Zone 5: the downstream recirculation region; and

- Zone 6: the boundary-layer development region.

These zones are displayed in Figure 1.1.

Lewis $(1975 a, 1975 b)$ first proposed this multiple-region approach to correlate the performance of enhanced tubes with the separation and reattachment mechanism. However, this farsighted approach was not further developed by subsequent researchers. 


\subsubsection{Variation in Local Heat-Transfer Coefficient}

Figures 7.1 and 7.2 show the normalized local Nusselt number ratios, $\mathrm{Nu} / \mathrm{Nu}$, as a function of the axial location with five different Reynolds numbers for Pr values of 0.71 and 21.7 , respectively. The particular tube selected for this comparison is the $02 / 40$ geometry $(\mathrm{e} / \mathrm{D}=0.02$ and $p / e=40$.). The performance at both rib sides is also shown by opening up or extending the $x$ axis. The vertical lines are used to separate the flow field into the six different zones. The four lines at the rib region are located at each of the four corners. With an increasing Pr, the following changes to the enhancement levels can be noted:

- Lower in the front recirculation zone for most of the $\mathrm{Re}$ range (zone 1),

- Substantially higher at the rib (zones 2,3 , and 4),

- Almost no change in the downstream recirculation zone for most of the $\mathrm{Re}$ range (zone 5), and

- Lower levels in the boundary-layer development zone (zone 6) for most of the Re range.

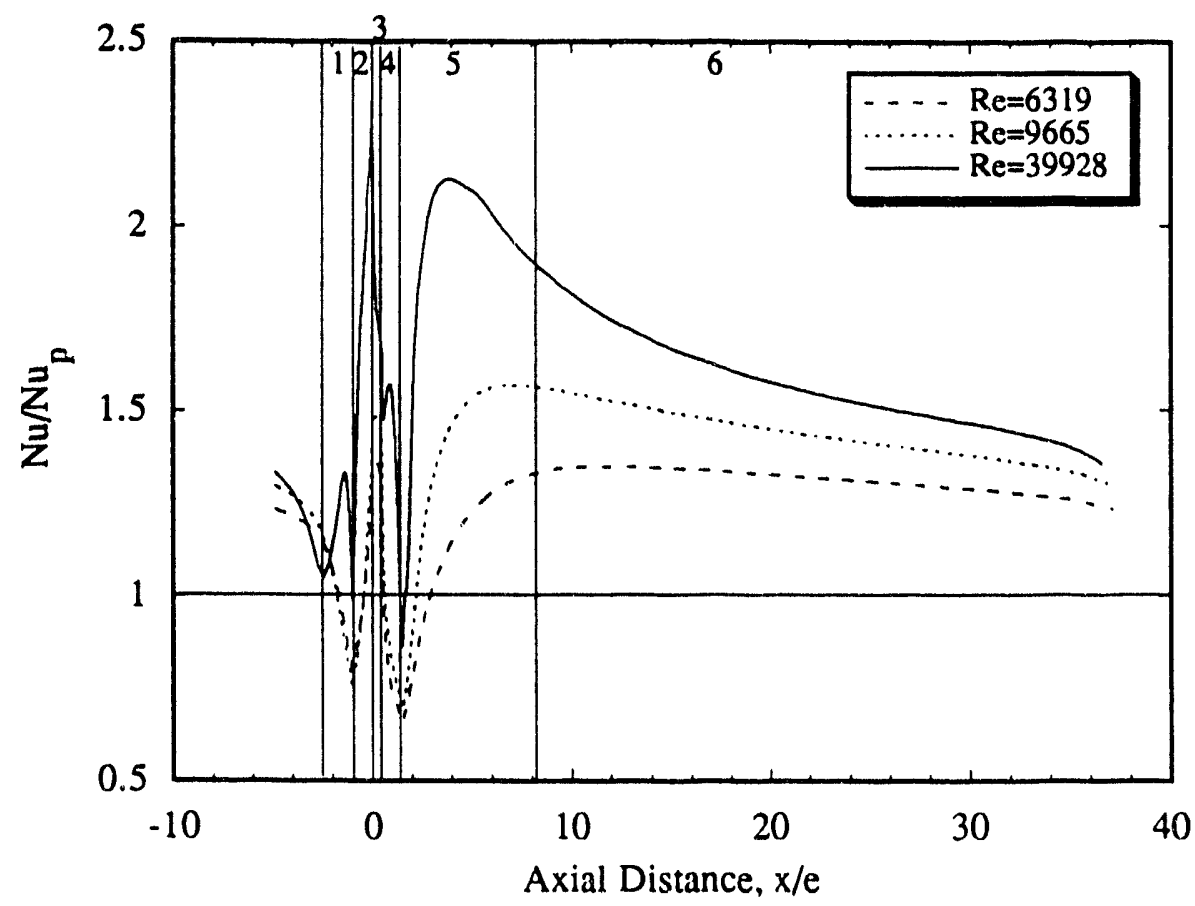

FIGURE 7.1 Local Nusselt Number Variation with Axial Distance for the $02 / 40$ Tube with $\mathrm{Pr}=0.71$ 


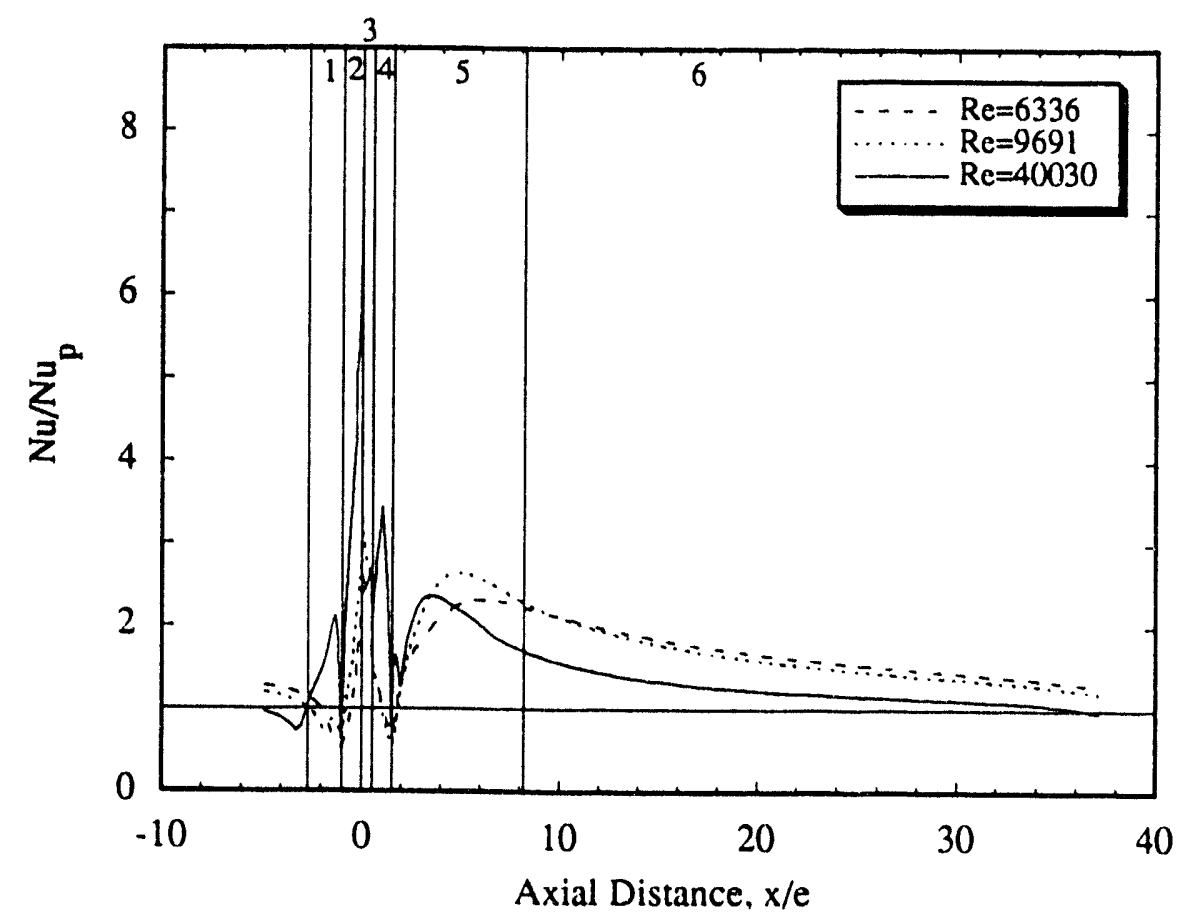

FIGURE 7.2 Local Nusselt Number Variation with Axial Distance for the $02 / 40$ Tube with $\mathrm{Pr}=21.7$

Figures 7.3 and 7.4 further illustrate the above findings; they are plots of local enhancement for three different Prandtl numbers at Reynolds numbers of about 6,300 and 40,000, respectively. Note the substantially higher performance at the rib and in the downstream recirculation region for the lower Reynolds number with an increase in the Prandtl number. This pattern, shown in Table 2.2, has been observed for the other four tube geometries considered for this study. The first important finding is that the impact on the thermal performance with an increasing Prandtl number is concentrated at the disruption or rib regions 2, 3, and 4.

These figures show that there are four maxima of the local heat-transfer coefficient. The two dominant maximums occur at the rib - the top leading edge - and in the downstream recirculation zone. The other secondary maximums occur only when the Reynolds numbers and/or the roughness Reynolds number, $\mathrm{e}^{+}$, exceed a transition value. Figures 7.2 and 7.4 show these secondary maximums at the rib area: one is located in the upstream separation bubble (zone 1), and the other is located on the vertical downstream face of the rib (zone 3). The magnitude of the enhancement levels at these secondary maximums also increases with the Prandtl number. The maximum on the vertical downstream face (zone 3 ) has not been observed for the $01 / 10$ geometry (apparently because of the smaller $\mathrm{e}^{+}$value). The authors are not aware of any experimental evidence that supports the existence of these secondary maximums. 


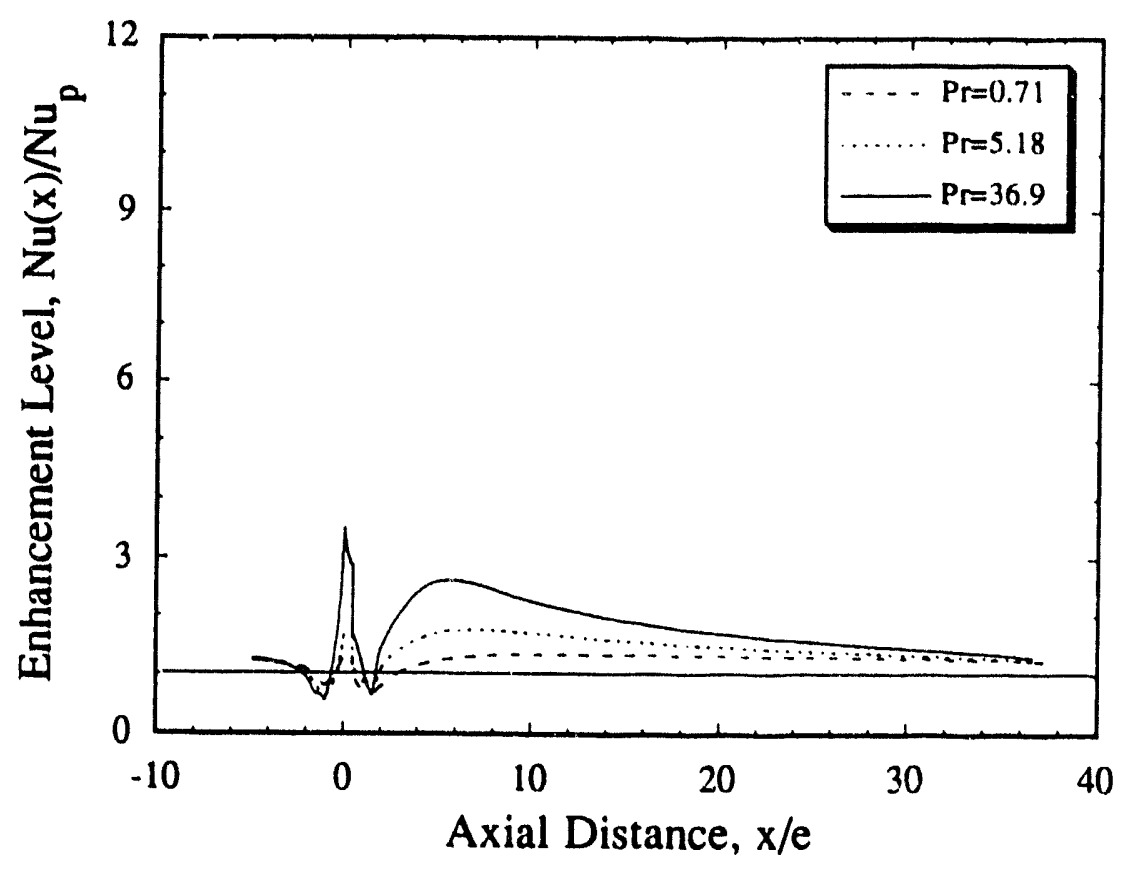

FIGURE 7.3 Local Nusselt Number Variation with Axial Distance for the 02/40 Tube with $R e=6,300$

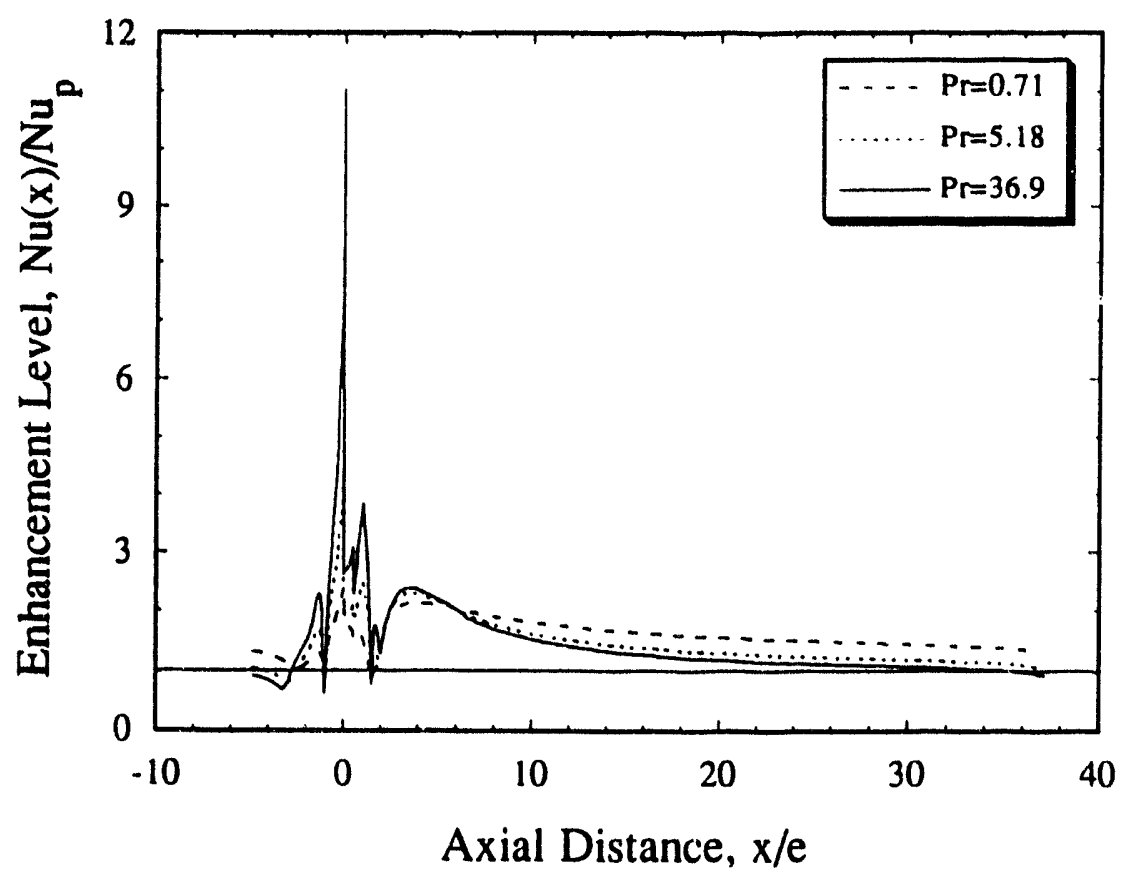

FIGURE 7.4 Loce: Nusselt Number Variation with Axial Distance for the 02/40 Tube with $R e=40,000$ 


\subsubsection{Variation in Maximum Entiancement Level at the Rib}

Because the Prandtl number influence is concentrated at the rib, it was decided to investigate the variation of the maximum enhancement level at this location in an attempt to explain the mean thermal performance shown in Figures 2.10 and 2.13. Figure 7.5 shows the maximum values, $\mathrm{Nu}_{\max } / \mathrm{Nu}$, at the rib as a function of the Reynolds number for $\operatorname{Pr}=0.71$ and 21.7 and for three tubes with the $p / e$ value of 10 . For air, the maximum values increase to asymptotic values with increasing Reynolds number. For the higher Pr fluid, these maximum values first increase, reach a maximum, and then decrease. The $R e$ value for the maximum depends on the e/D value: the higher the $\mathrm{e} / \mathrm{D}$ value, the lower the Reynolds number required for the maximum. This pattern is very similar to that shown in Figures 2.10 and 2.13 .

The maximum enhancement-level data for the $01 / 10,02 / 10,04 / 10,02 / 20$, and $02 / 40$ geometries are plotted with $\mathrm{e}^{+}$in Figures 7.6, 7.7, 7.8, 7.9, and 7.10, respectively. The influence of the Prandtl number on the shape of these curves is identical to that shown in Figures 2.10 and 2.13. These figures reveal that the variations of the mean enhancement levels and the maximum enhancement levels at the rib follow identical patterns with changes of the Prandtl number and the roughness Reynolds number.

The actual mechanisms that are responsible for this enhancement-level variation with the Prandtl number at the rib region are very complicated and merit further investigation. In particular, the validation of the turbulence model to capture the flow details has not been demonstrated, although it did predict the local heat-transfer-coefficient variations for air. However, a possible explanation is as follows. The flow field at the three rib surfaces consists of separations and reattachments. For these flows, it has been established that the heat-transfer-coefficient dependence on the Reynolds number is in the $0.65-0.75$ range. Some flow conditions that support this statement are cross flow to a single tube and impinging jets. Another example is the measured mean mass-transfer coefficients obtained with the naphthalene method $(\operatorname{Pr}=2.5)$ for the three faces of a wall-mounted cube (Chyu and Natarajan 1991). The exponents ranged from 0.626 to 0.661 . For flow inside a plain tube (a boundary-layer flow), Figures 2.11 and 2.12 show that the exponent on the Reynolds number is in the 0.65-0.75 range for air and in the 0.80-0.85 range for water and higher Prandtl number fluids.

The effect of the flow rate on the enhancement level at the rib is now addressed. The flow rate increases with increasing $\mathrm{Re}$ or $\mathrm{e}^{+}$because of the turbulence caused by the rib penetration of the laminar sublayer - a thinning of the laminar sublayer. This decrease of the sublayer thickness is similar to that obtained for three-dimensional roughness, as shown in Figure 7.8 (sce Hinze [1959]). An increase of the enivancement level in both the downstream recirculation and boundarylayer development regions also occurs, as shown in Figure 7.3. With a further increase, the separation and reatiachment flow characteristics begin to dominate with the two dominant maximums at the rib and in the downstream recirculation region. For air, the enhancement level continues to increase and reaches an asymptotic value because of comparable Reynolds number exponents. However, for fluids with $\operatorname{Pr}>1$, the enhancement level begins to decrease becaus: the exponents for reattaching flows are less than for boundary-layer flows. 


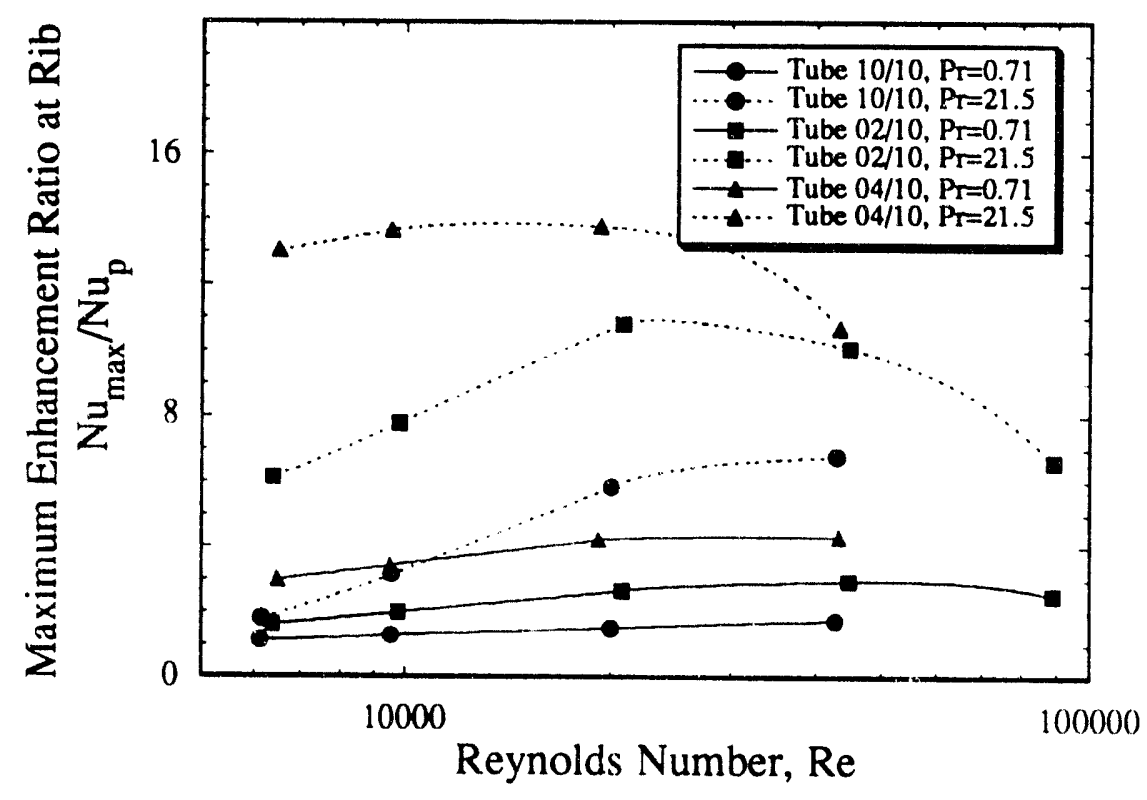

FIGURE 7.5 Maximum Nusselt Number Variation at the Rib for Three Prandil Numbers and Three Tubes with the p/e Value of 10

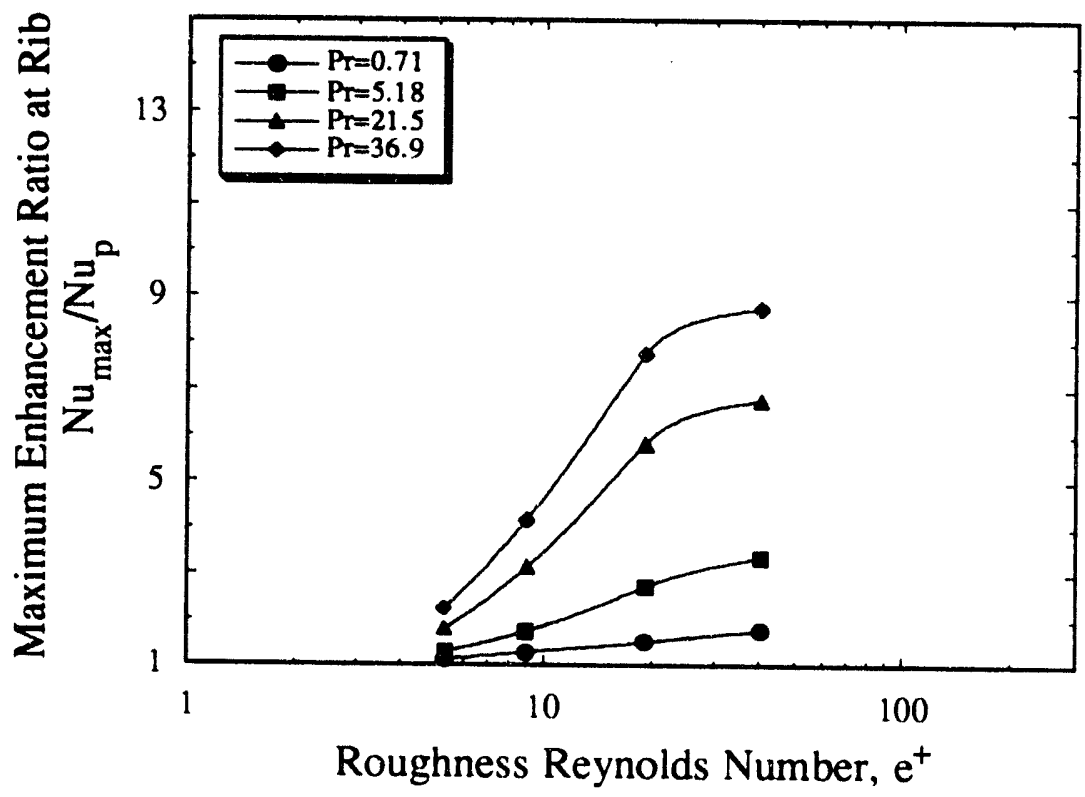

FIGURE 7.6 Maximum Enhancement-Level Variation at the Rib as a Function of the Roughness Reynolds Number with the Prandtl Number as a Parameter for the 01/10 Tube Geometry 


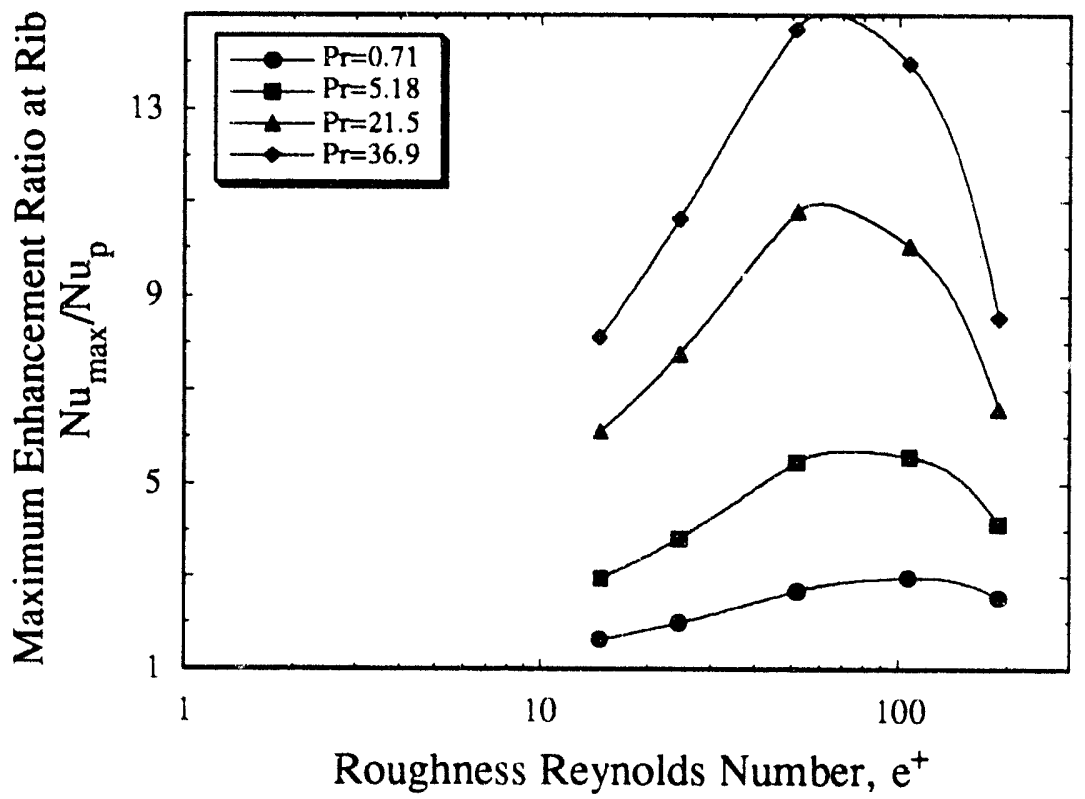

FIGURE 7.7 Maximum Enhancement-Level Variation at the Rib as a Function of the Roughness Reynolds Number with the Prandtl Number as a Parameter for the 02/10 Tube Geometry

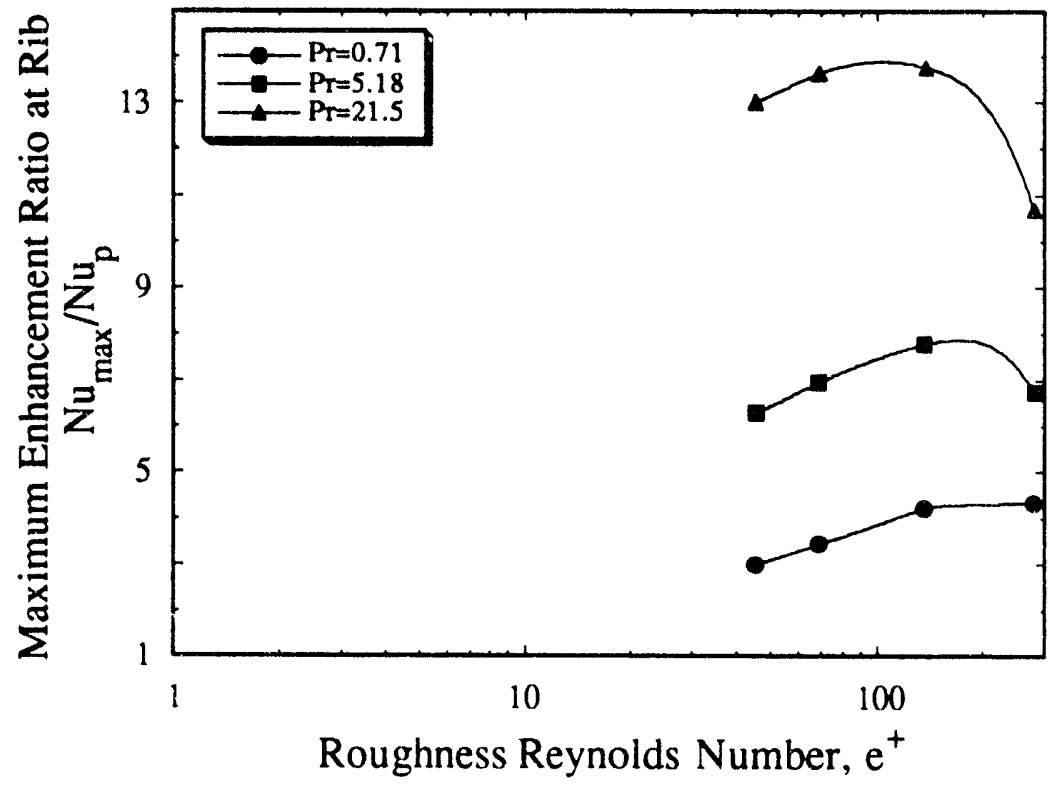

FIGURE 7.8 Maximum Enhancement-Level Variation at the Rib as a Function of the Roughness Reynolds Number with the Prandtl Number as a Parameter for the 04/10 Tube Geometry 


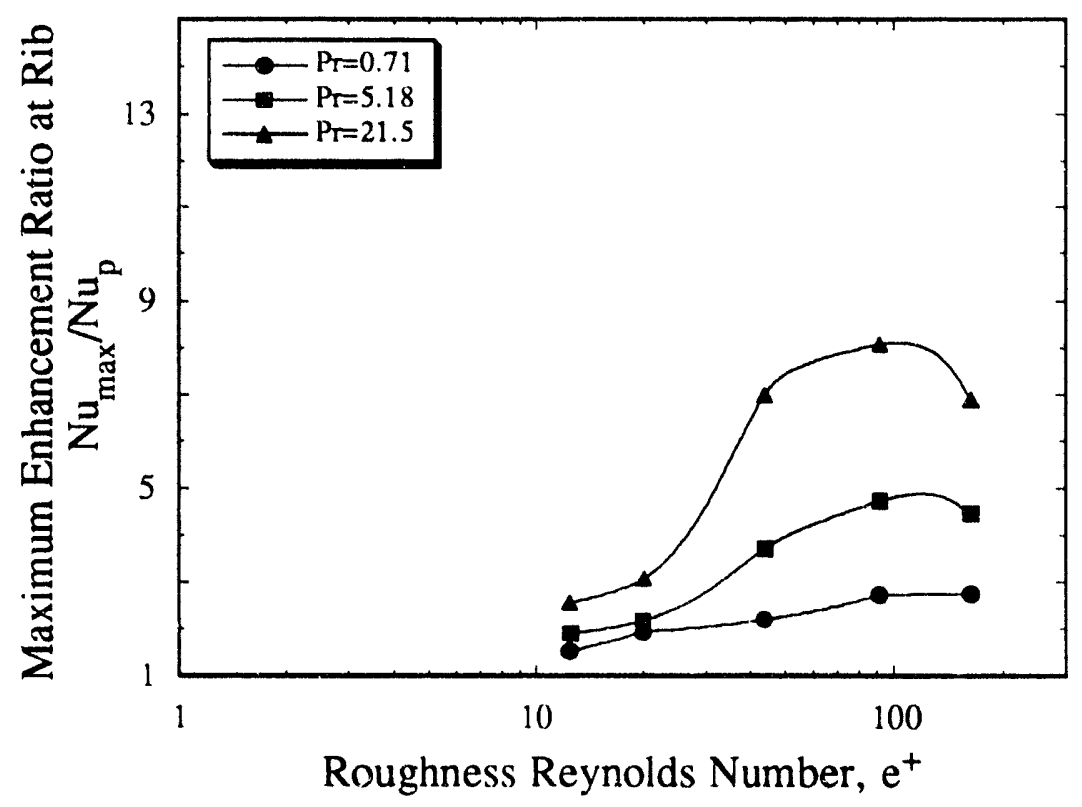

FIGURE 7.9 Maximum Enhancement-Level Variation at the Rib as a Function of the Roughness Reynolds Number with the Prandtl Number as a Parameter for the 02/20 Tube Geometry

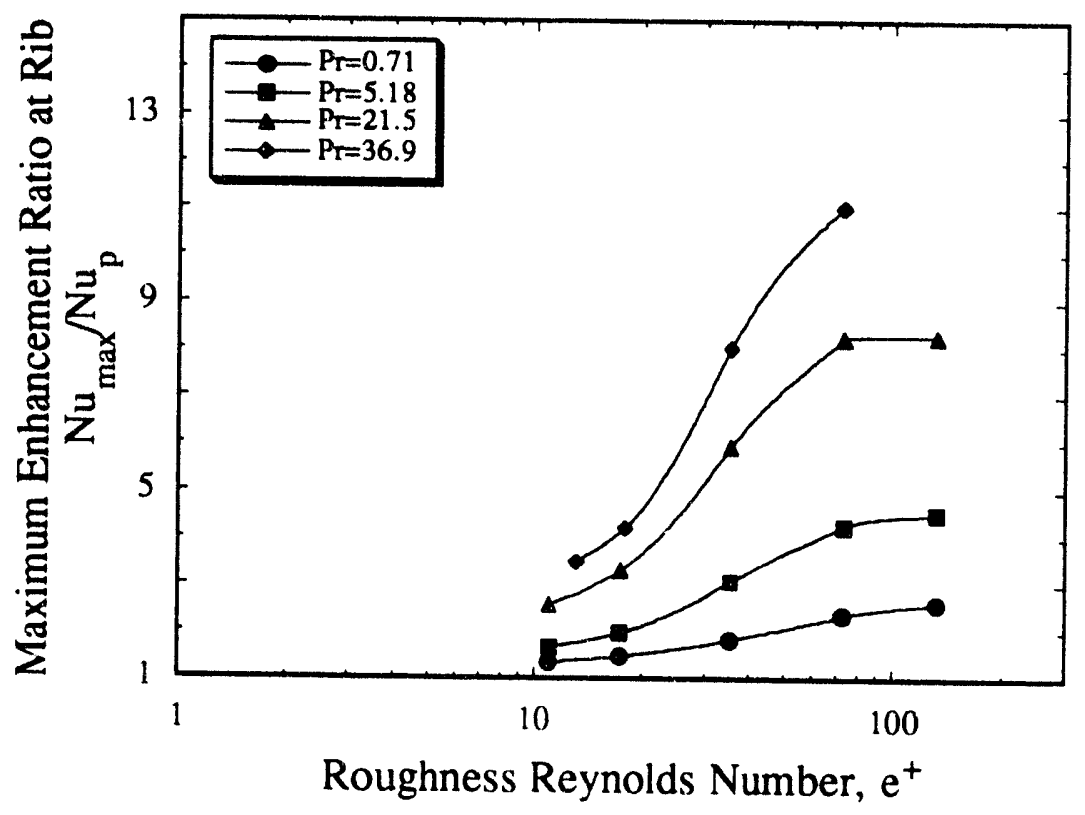

FIGURE 7.10 Maximum Enhancement-Level Variation at the Rib as a Function of the Roughness Reynolds Number with the Prandtl Number as a Parameter for the 02/40 Tube Geometry 


\subsubsection{Influence of the RIb PItch on the RIb Thermal Performance}

The maximum heat transfer at the rib decreases with an increase of the pitch or spacing of the disruptions; however, the magnitude of this decrease depends on the Prandtl number. Figure 7.11 shows the maximums, $\mathrm{Nu}_{\max } / \mathrm{Nu}_{\mathrm{p}}$, as a function of the Reynolds number for two Prandtl number fluids and the $02 / 10,02 / 20$, and 02/40 tube geometries. Note the almost identical values for air but an increased departure for the larger Pr fluid. The explanation is that the impact of the upstream rib decreases with an increased pitch or an elongated boundary-layer development region. However, the sensitivity to the perturbations of the flow caused by the upstream rib increases with an increasing Prandtl number.

For lower Pr fluids, the mean thermal performance is dependent on the pitch, although it has a small effect on the thermal performance at the rib. For air and, to a lesser extent, water, the downstream recirculation zone and the boundary-layer development zones make a major contribution to the mean thermal performance. The influence of the Prandtl number on the thermal performance in the downstream recirculation zone is discussed in the next section.

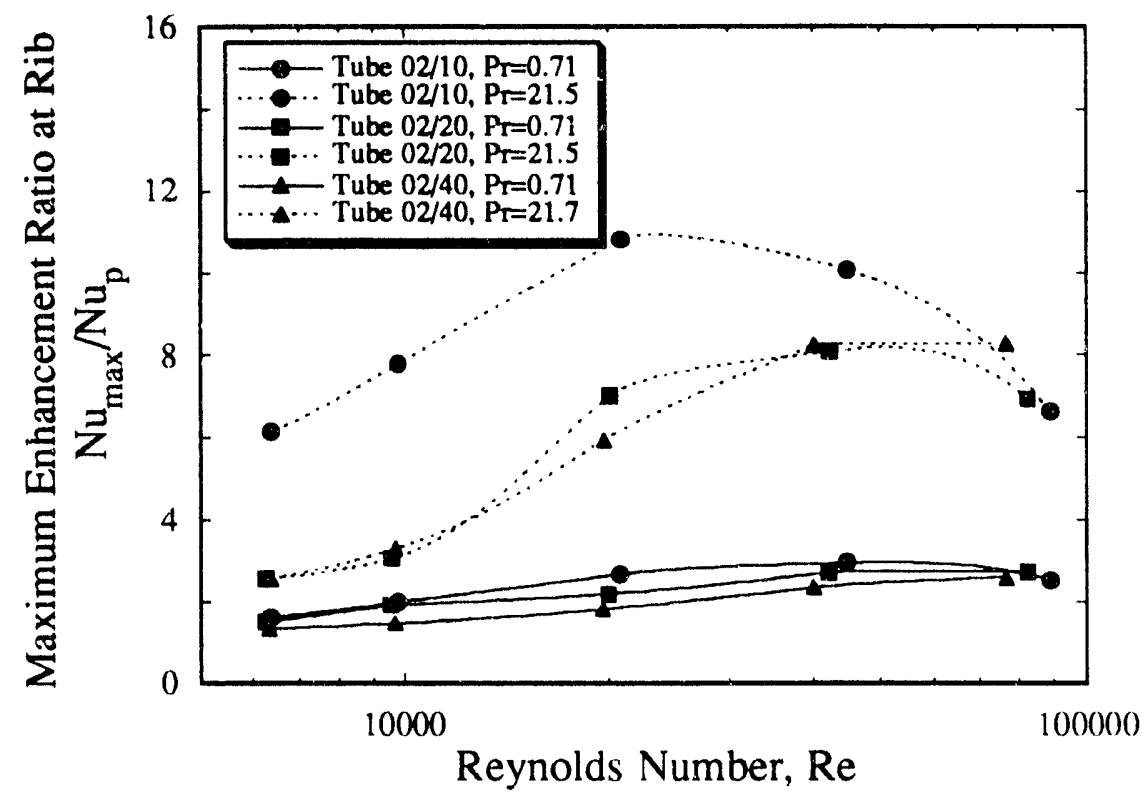

FIGURE 7.11 Maximum Heat Transfer at the Rib as a Function of the Reynolds Number with the Prandtl Number as a Parameter for the 02/10,02/20, and 02/40 Tube Geometries 


\subsubsection{Thermal Performance In the Downstream Recirculation Region}

A major contribution to the total heat transfer comes from the downstream recirculation region, or zone 5. For low Pr fluids and a low Reynolds number, this contribution is dominant, as shown in Figures 7.1 and 7.3, respectively. This section discusses the magnitude of the heat transfer in the downstream recirculation region and the location of the maximum heat-transfer coefficient.

\subsubsection{Magnitude of the Maximum Heat Transfer}

Figure 7.12 shows $\mathrm{Nu}_{\max } / \mathrm{Nu}_{\mathrm{p}}$ as a function of the Reynolds number for $\operatorname{Pr}=0.71$, $\operatorname{Pr}=21.7$, and the $02 / 10,02 / 20$, and $02 / 40$ tube geometries. For air, the contributions to the total increase with the Reynolds number and reach asymptotic values. For $\operatorname{Pr}=21.7$, maximums are again obtained. As expected, this characteristic is identical to that of the mean enhancement level and the maximum enhancement level at the rib. An extensive review of reattaching flows (Ota and Nishiyama 1987) has established that the maximum local-heat-transfer-coefficient dependencies, as expressed by the exponents on the Reynolds and Prandtl numbers, are about 0.67 and 0.33 , respectively. As a result, the influence of the Prandtl number on the thermal performance in the downstream recirculation zone is similar to that at the rib.

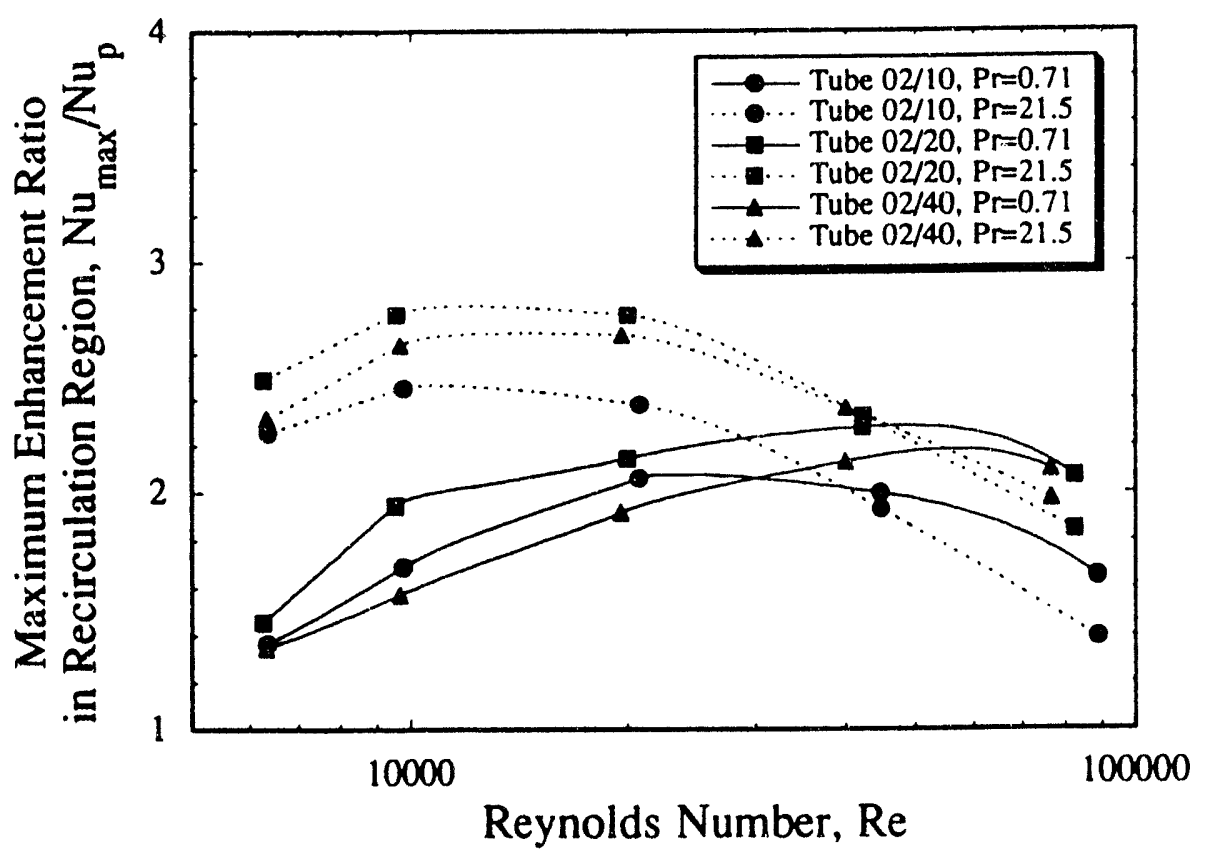

FIGURE 7.12 Magnitudes of the Maximum Heat Transfer in the Downstream Recirculation Region as a Function of the Reynolds Number for $\operatorname{Pr}=0.71, \operatorname{Pr}=21.7$, and the $02 / 10,02 / 20$, and 02/40 Tube Geometries 
The major finding of this investigation is that the mean thermal performance, as displayed by Figures 2.10 and 2.13, is the manifestation of the performance characteristics that occur at the two points of maximum heat transfer - at the rib and in the downstream recirculation zone.

Two possible reasons could account for the heat-transfer enhancement in the downstream recirculation zone:

1. The thickness of the thermal boundary layer decreases and the temperature gradient increases as a result of the flow impacting the surface near the reattachment point.

2. The temperature gradient is increased as a result of the high levels of turbulence near the surface.

Experimental evidence has been presented by Mori and Daikoku (1972) that conclusively proves that the latter is the correct reason. This point can be further substantiated with results from the numerical model. Figure 7.13 shows the turbulent-kinetic-energy variation $\left(\mathrm{k}^{+}\right)$in the radial direction at various locations downstream from the rib for the 02/20 tube geometry. Note the very high values of $\mathrm{k}^{+}$in the $\mathrm{x} / \mathrm{e}=2.6-5.3$ range within the downstream recirculation zone. As shown in the following section, the location of the maximum turbulent kinetic energy is near the locations of the maximum heat transfer.

\subsubsection{Location of the Maximum Heat Transfer}

This section presents a comparison of the locations of the maximum heat transfer, the maximum wall shear stress, and the reattachment point. The locations of the maximum heat transfer in the recirculation regions were obtained from plots similar to those shown in Figures 7.1 through 7.4. The locations of the maximum wall shear stress and the reattachment points were obtained from the plots of the wall shear stress shown in Figures 7.14 to 7.18 for all five tube geometries considered by Webb et al. (1971). The wall shear stress at the reattachment point is equal to zero.

Figures 7.19 and 7.20 show these characteristic lengths expressed as multiples of the rib height for the $02 / 20$ and $02 / 40$ iubes, respectively. Note the following:

1. The location of the maximum heat transfer in the downstream recirculation region is located between the reattachment length and the location of the maximum shear stress.

2. The location of the maximum heat transfer moves toward the upstream rib as the Reynolds and Prandtl numbers are increased. 

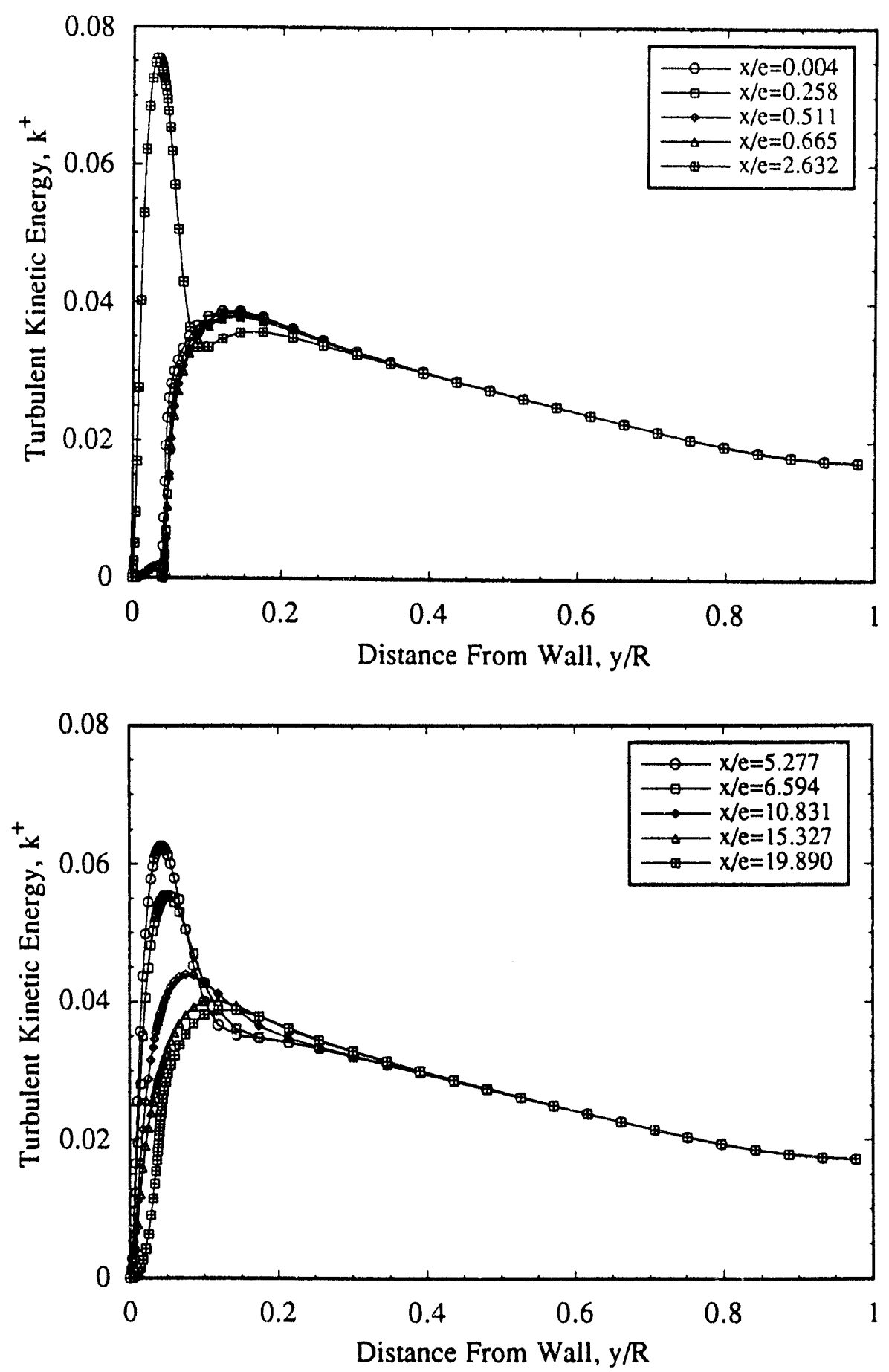

FIGURE 7.13 Turbulent Kinetic Energy Variation with Distance from the Wall at Various Axial Locations 


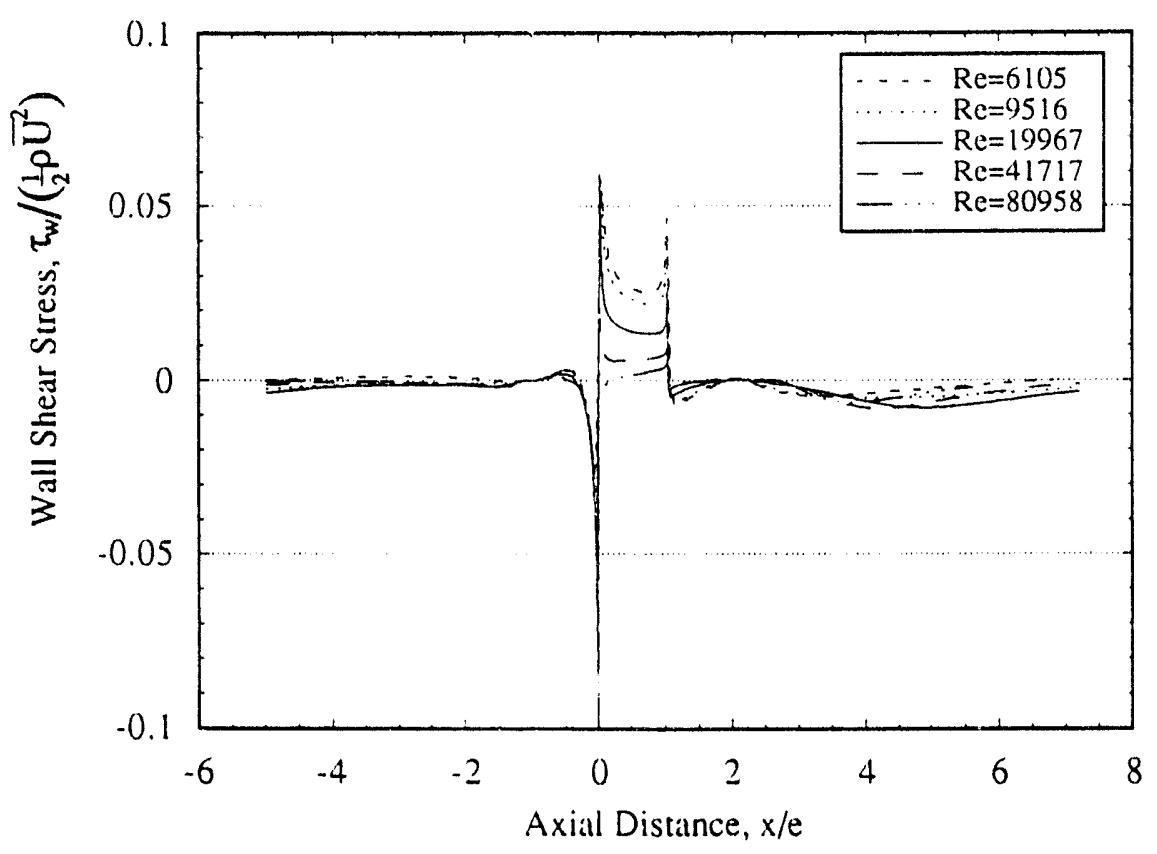

FIGURE 7.14 Local Wall Shear Stress as a Function of the Axial Location for the $01 / 10$ Tube Geometry

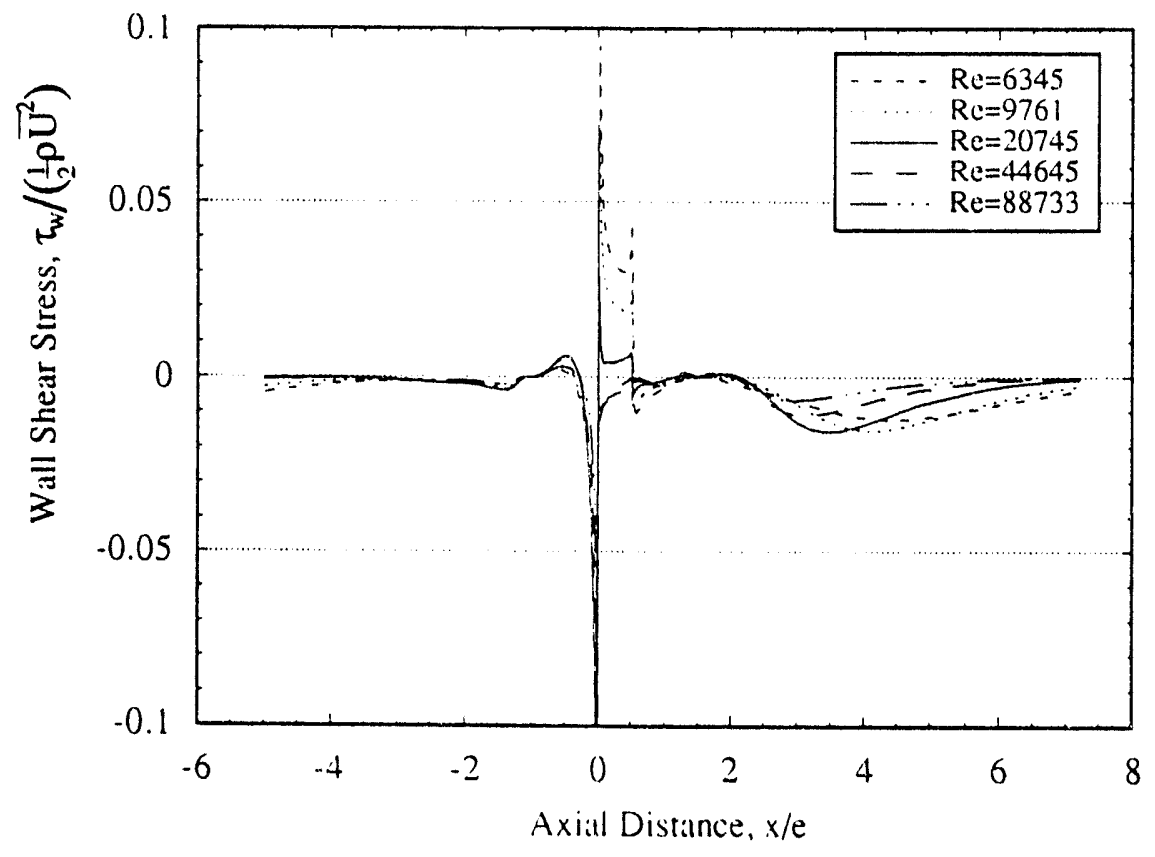

FIGURE 7.15 Local Wall Shear Stress as a Function of the Axial Location for the 02/10 Tube Geometry 


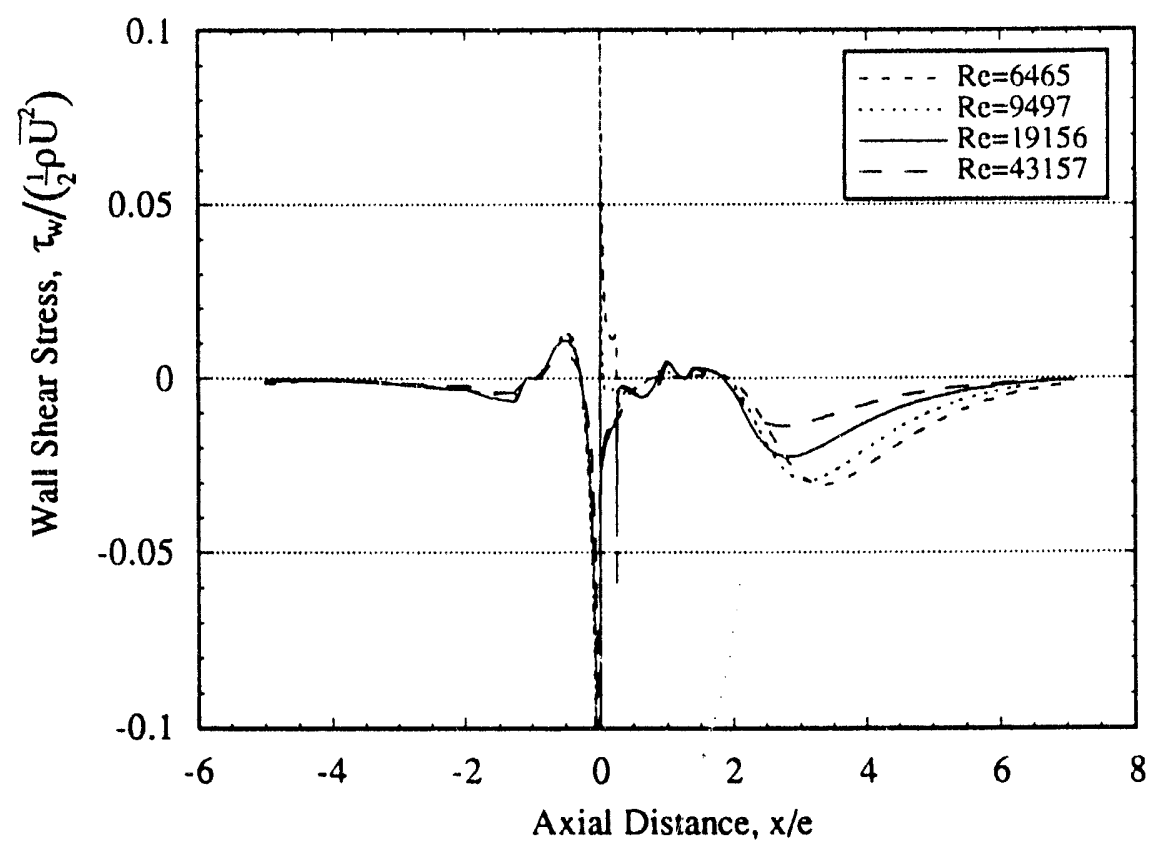

FIGURE 7.16 Local Wall Shear Stress as a Function of the Axial Location for the 04/10 Tube Geometry

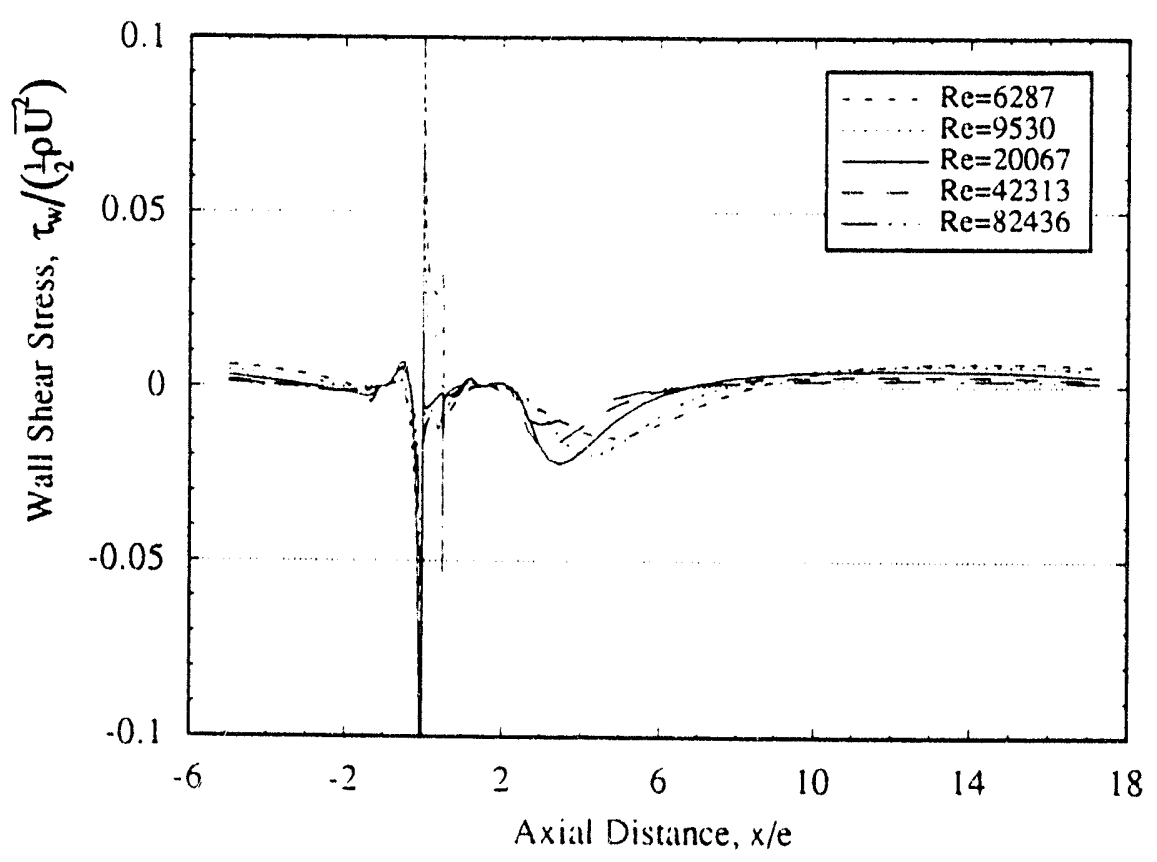

FIGURE 7.17 Local Wall Shear Stress as a Function of the Axial Location for the 02/20 Tube Geometry 


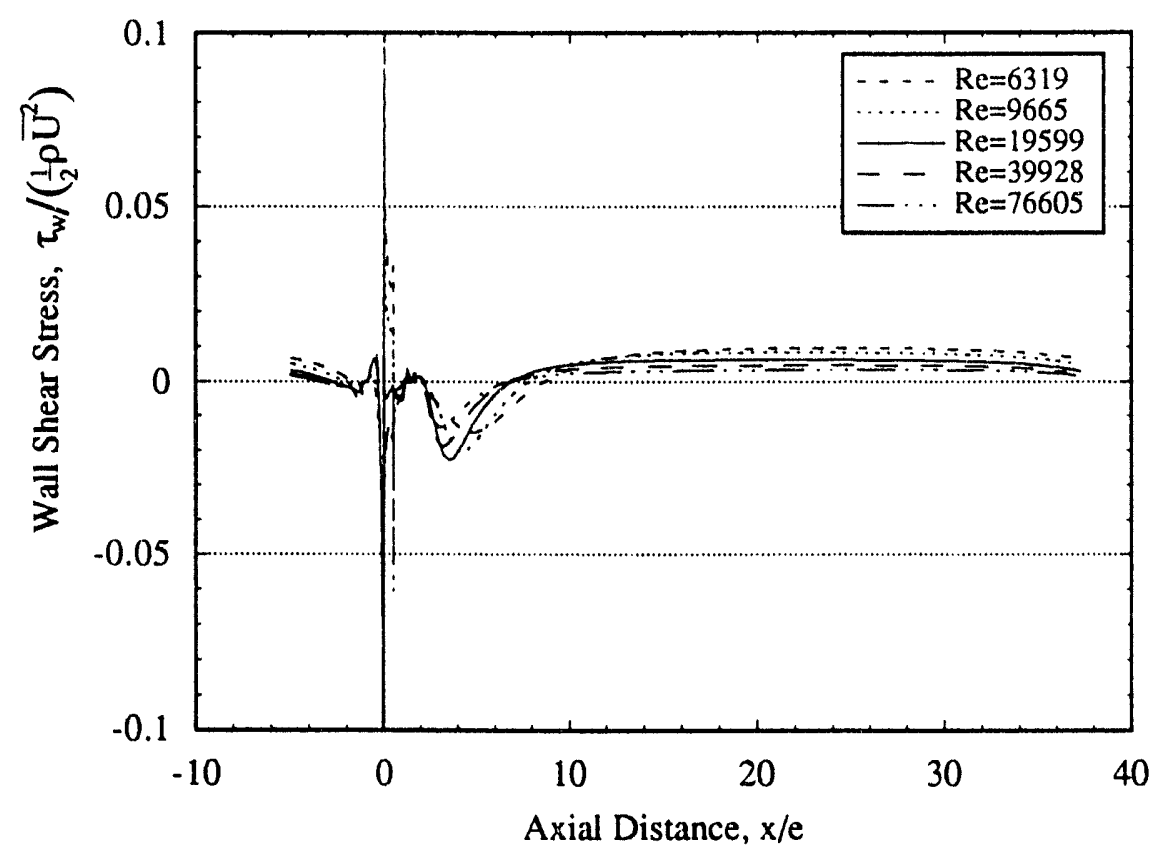

FIGURE 7.18 Local Wall Shear Stress as a Function of the Axial Location for the 02/40 Tube Geometrv

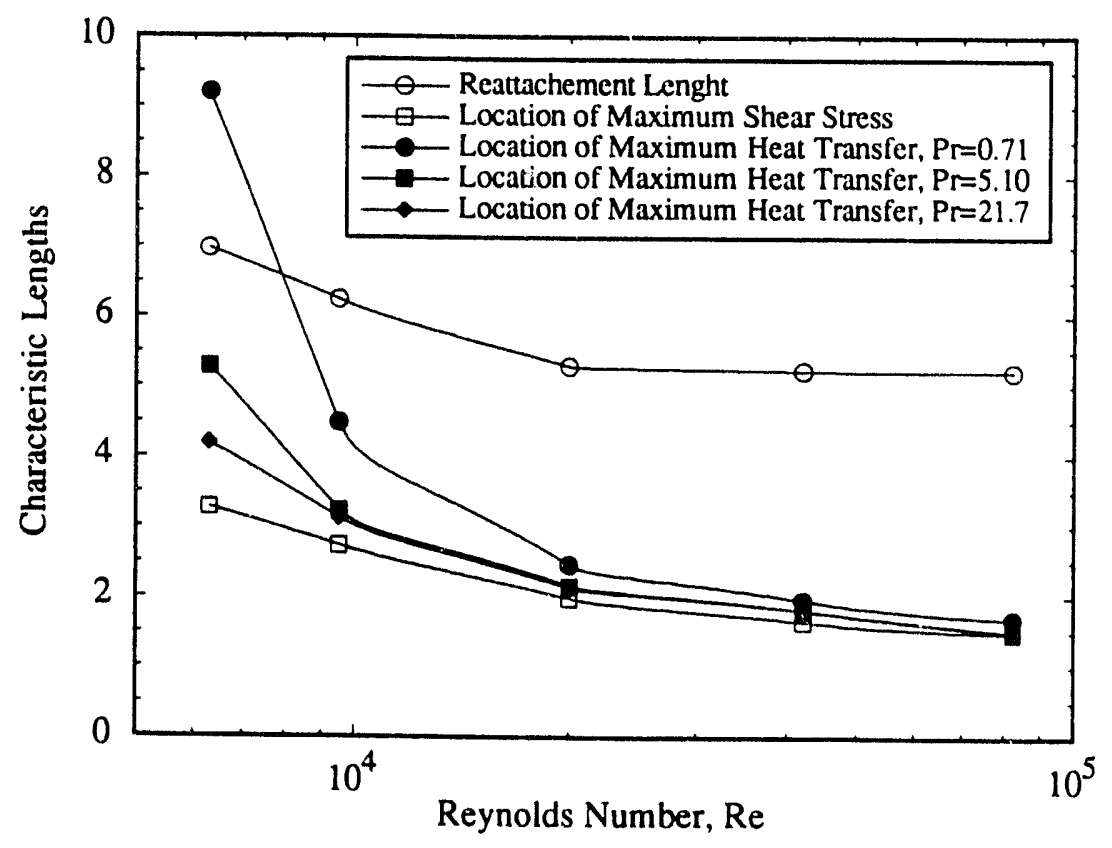

FIGURE 7.19 Characteristic Lengths in the Downstream

Recirculation Region for the 02/20 Tube Geometry 


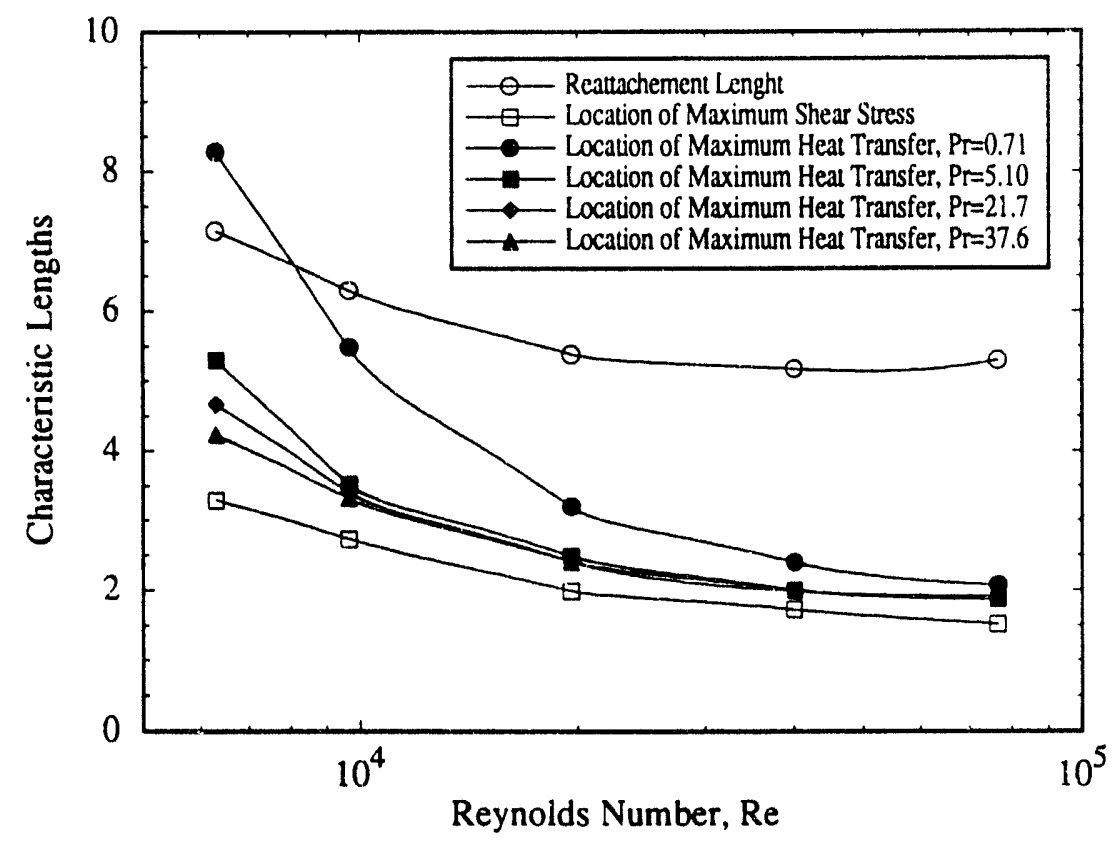

FIGURE 7.20 Characteristic Lengths in the Downstream Recirculation Region for the 02/40 Tube Geometry

A minor exception to the first finding is that the location of the maximum heat transfer exceeds the reattachment length for Reynolds numbers less than about 8,000.

The variations of the locations of the maximum heat transfer and the reattachment point for tube geometries that are characteristic of the separation and reattachment mechanism have been determined by previous experimental studies (Hijikata et al. 1987; Hijikata and Mori 1987; Berger and Hau 1979; Baughn and Roby 1992). However, these studies did not investigate the influence of the Prandtl number. The studies by Hijikata et al. (1987) and Hijikata and Mori (1987) did show that the location of the maximum heat transfer is upstream of the reattachment point and that the reattachment length for an arc-shaped disruption decreased with increasing Reynolds number. The experimental results obtained by Berger and Hau (1979) for very large Schmidt numbers showed that the location of the maximum heat transfer moves forward with increasing Reynolds number with rectangular-shaped disruptions. However, when Baughn and Roby (1992) used air as a test fluid, they did not observe any movement of the location of the maximum hent-transfer point for a broad range of Reynolds numbers. 
Figures $7.21,7.22$, and 7.23 are similar plots of the characteristic lengths tor the $01 / 10$, $02 / 10$, and $04 / 10$ tubes, respectively. The common features between these figures and Figures 7.19 and 7.20 are as follows:

1. The locations of the maximum heat transfer decrease with the Reynolds number and Prandti number. and

2. The lower bound is the location of the maximum wall shear stress.

Note that the reattachment length is not shown in Figures 7.21 to 7.23 . For the tubes with $p / e=10$, there were no reattachment points, or the wall local shear-stress values did not become positive after the rib, as shown in Figures 7.14, 7.15, and 7.16 for the 01/10, 02/10, and 04/10 tubes, respectively. The authors were unable to find any experimental data to confirm or contradict this somewhat surprising finding. The common belief was that the reattachment point always existed near the point of maximum heat transfer, even fo: $p / e$ values greater than about eight.

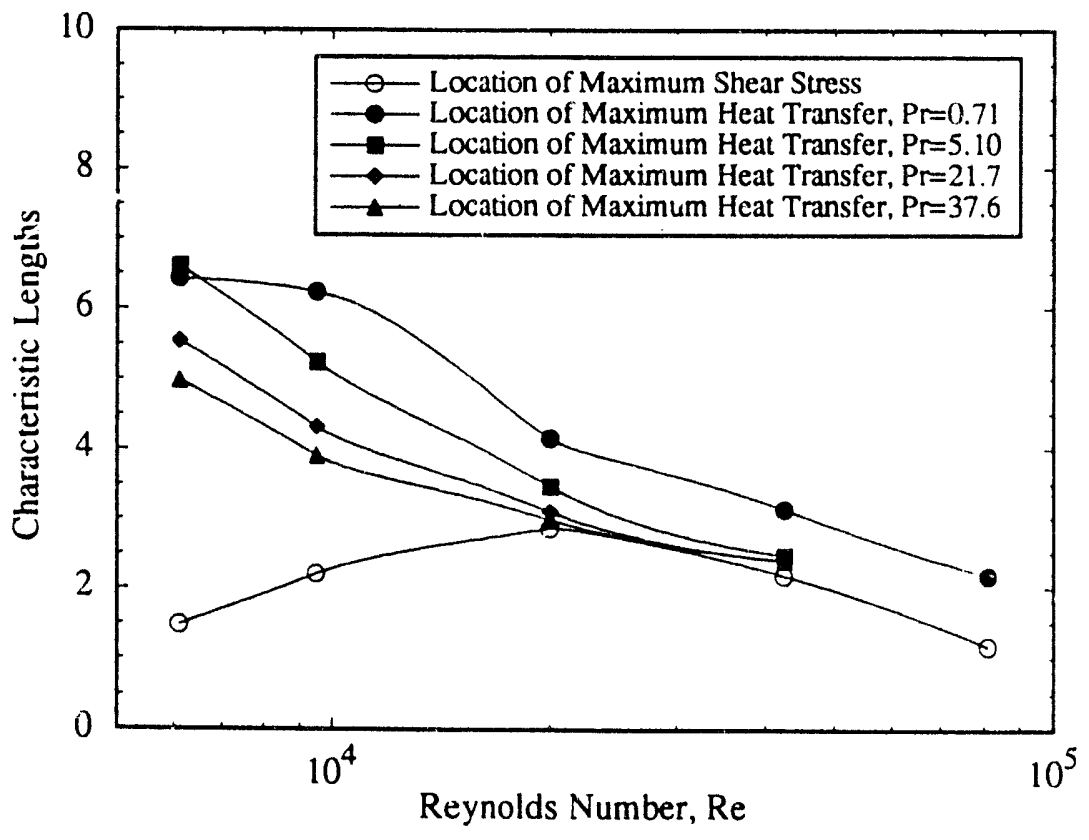

FIGURE 7.21 Characteristic Lengths in the Downstream Recirculation Region for the 01/10 Geometry 


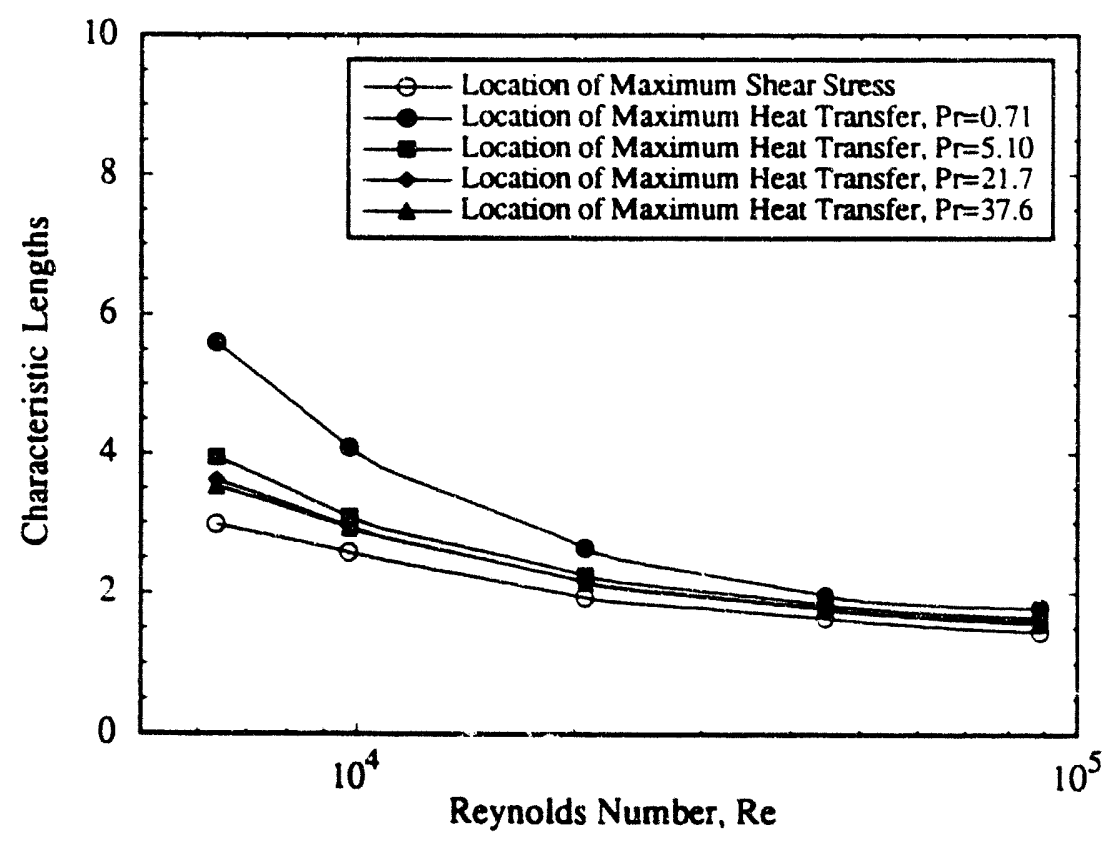

FIGURE 7.22 Characteristic Lengths in the Downstream Recirculation Region for the 02/10 Tube Geometry

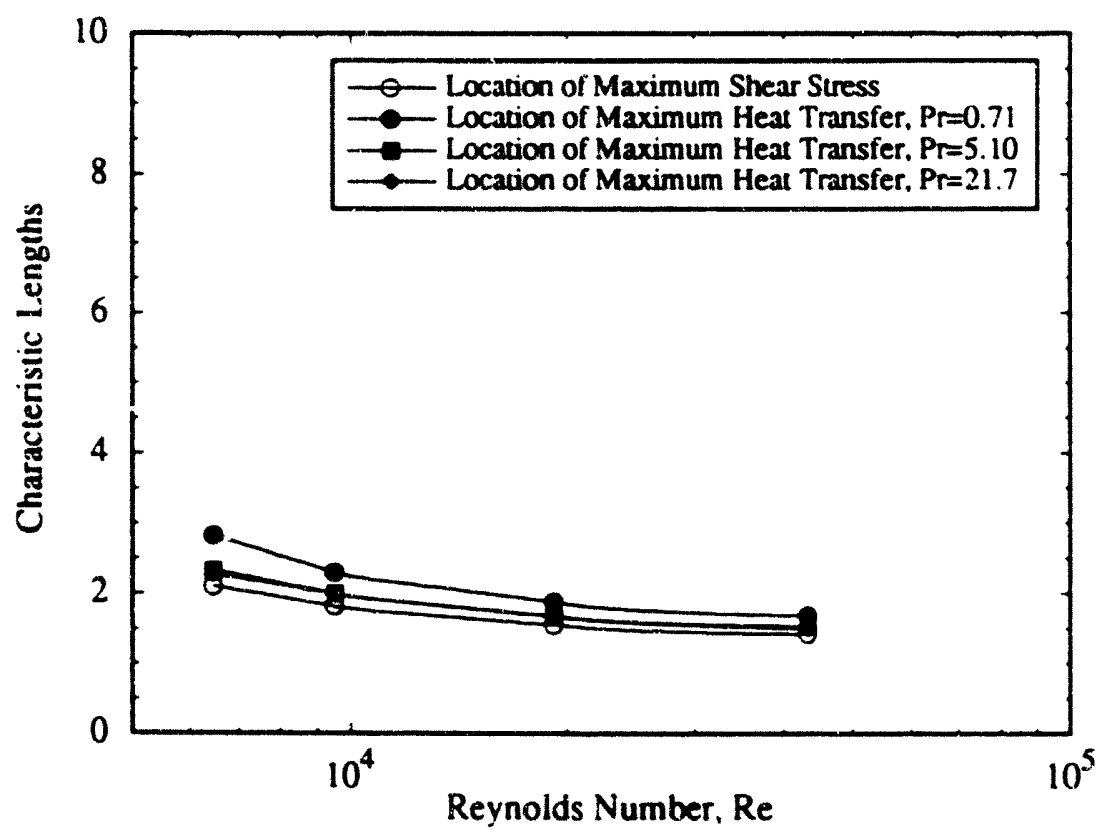

FIGURE 7.23 Characteristic Lengths in 'he Downstream Recirculation Region for the 04/10 Tube Geometry 
Additional experimental studies have presented local heat-transfer data for different Reynolds and Prandtl numbers, but for a single disruption. Krall and Sparrow (1966) and Sydberger and Lotz (1982) determined the local maximums downstream of orifice-type restrictions in tubes; Krall and Sparrow (1966) used water and Sydberger and Lotz (1982) used electrochemical measurements (Schmidt number of 1,460). Both studies revealed an upstream movement of the maximums with increasing Reynolds number. Krall and Sparrow (1966) also compared the results obtained at two Prandtl numbers, $\operatorname{Pr}=3.0$ and 6.0. For this comparison, it was not clear whether the Prandtl numbers had a consistent impact on the location of the maximum heat-transfer coefficient. Sydberger and Lotz (1982) stated that the location of the maximum mass transfer was well within the downstream recirculation region, which agrees with the results shown in Figures 7.19 and 7.20 for large Prandtl numbers.

The above discussion clearly identifies how the Prandtl number influences the thermal performance of tubes with the separation and reattachment enhancement mechanism. In the next section, the body-fitted numerical code will be used to investigate the effect of different shapes for this same enhancement mechanism.

\subsection{Effects of the Disruption Shape}

This section of the report describes the effects of the disruption shape on the pressure-drop and the heat-transfer performance of enhanced tubes with air as the fluid of interest $(\operatorname{Pr}=.71)$. Figure 7.24 shows the four different disruption shapes that are considered in this investigation: sine, semicircle, arc, and trapezoid. The nonorthogonal, body-fitted numerical code is used for the following runs. These final runs are performed with the convective-differencing scheme of Zhu (1991) and the two-layer model of Chen and Patel (1988).

\subsubsection{Wall Shear Stress and Friction Factors}

Figure 7.25 shows the normalized local wall shear stresses (i.e., skin-friction coefficients) for a sine-shaped disruption. The reattachment-point location - the point of zero shear stress moves toward the rib with an increasing Reynolds number. A similar finding was obtained for the

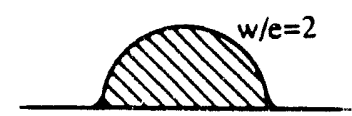

Semicircle

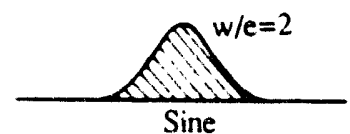

FIGURE 7.24 Different Disruption Shapes

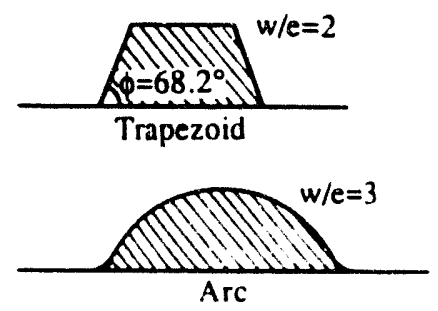

Considered 


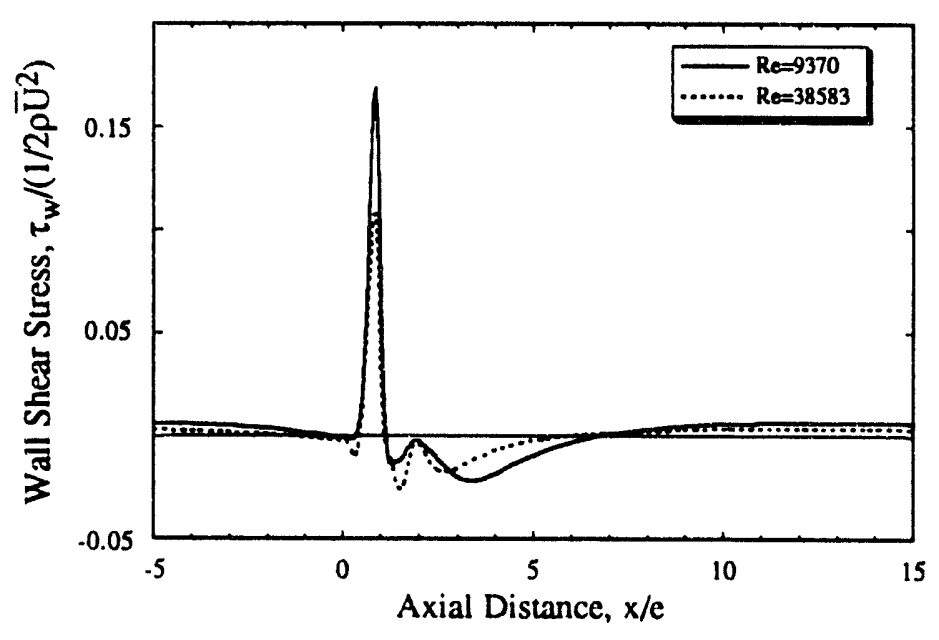

FIGURE 7.25 Normalized Wall Shear-Stress Distributions for a Sine-Shaped Disruption: $p / e=20$ and $w / e=2$

other three disruption shapes. 'This finding is in agreement with that observed by Hijikata et ai. (1987) and presented in Figure 2.7. It should be noted that the size of the upstream recirculation zone increases with an increasing Reynolds number. Figure 7.25 also shows that the upstream recirculation region is much smaller than the downstream recirculation region. There is a sharp increase in the wall shear stress on the leading face of the rib and a flow reversal on the rib just immediately after this pike. The strength of this flow reversal increases with an increasing Reynolds number. Figures 7.26 and 7.27 compare the wall shear stress for four different shapes at Reynolds numbers of about 9,400 and 39,000 , respectively. The wall shear-stress patterns in the vicinity of the rib are strongly shape dependent. The wall shear stress of the trapezoidal rib exhibits two maxima at the leading and trailing top corners. Also, there is no flow reversal on the rib. The other three shapes show flow reversals on the contoured trailing faces. These figures demonstrate that the size of the upstream recirculation region is not strongly dependent on the shape of the disruption; however, the size of the downstream recirculation region is more dependent on the disruption shape.

Table 7.1 presents the reattachment point locations that are defined as the distance from the end of the disruptions to the point of zero wall shear stress. Table 7.1 also provides friction factors for the different disruption shapes for two different spacings $(p / e=10$ and 20$)$ and for two different Reynolds numbers. This table shows that both the reattachment lengths and friction factors decrease as the shape becomes less contoured - trapezoid, sine, semicircle, and arc. The influence of the shape becomes more pronounced with decreasing pitch and increasing Reynolds number. 


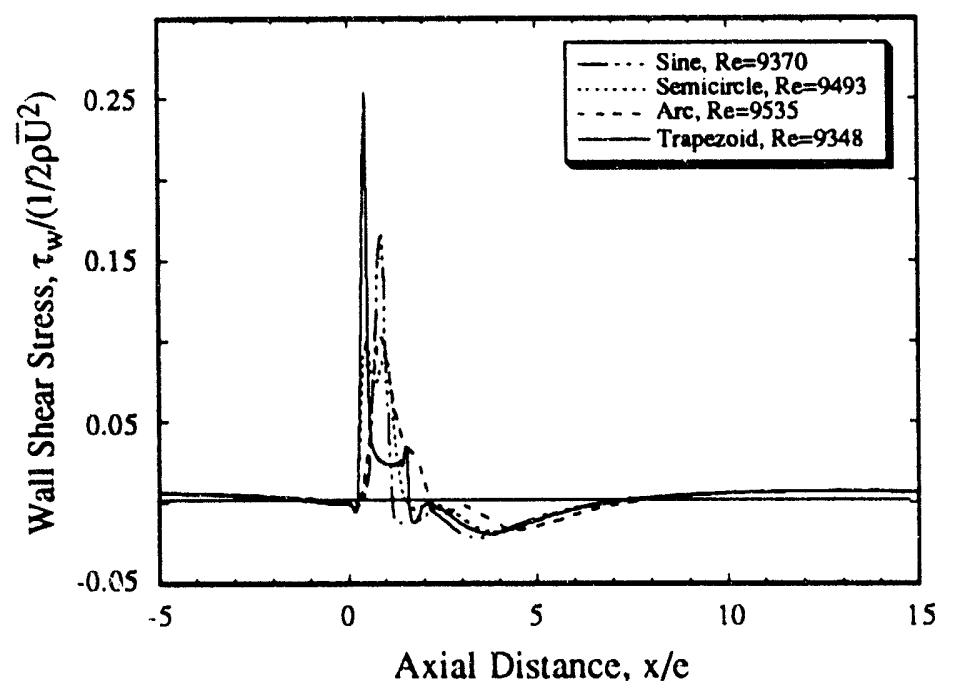

FIGURE 7.26 Comparison of the Wall ShearStress Variations for the Smaller Reynolds Number: $p / e=20$

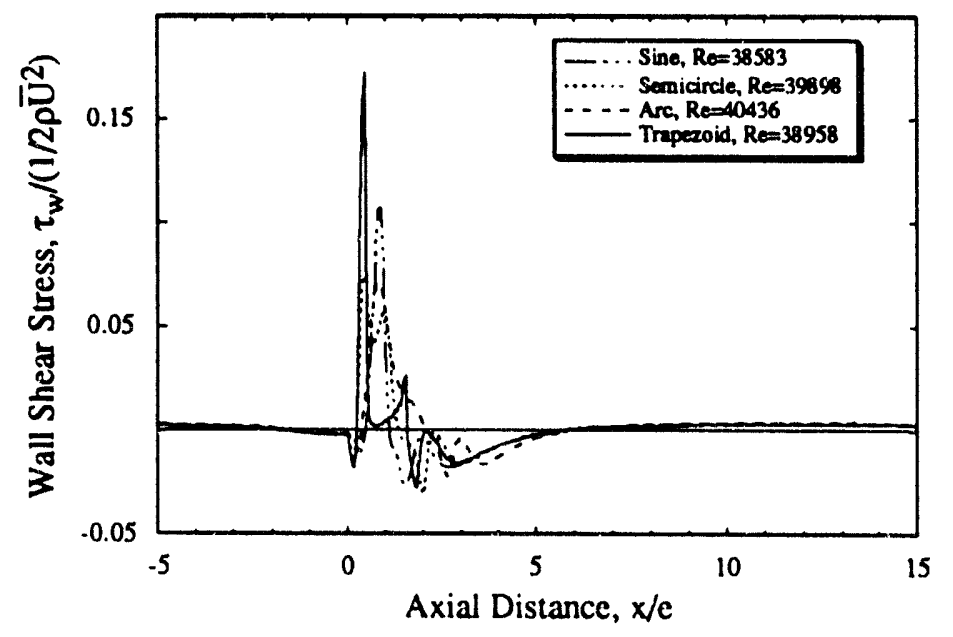

FIGURE 7.27 Comparison of the Wall ShearStress Variations for the Larger Reynolds Number: $p / e=20$ 
TABLE 7.1 Reattachment Lengths and Friction Factors

\begin{tabular}{|c|c|c|c|c|c|}
\hline \multirow[b]{3}{*}{ Shape } & \multirow[b]{3}{*}{$p / \theta$} & \multirow{2}{*}{\multicolumn{2}{|c|}{$\begin{array}{l}\text { Reattachment Lengths } \\
\text { Reynolds Number } \\
\end{array}$}} & \multirow{2}{*}{\multicolumn{2}{|c|}{$\begin{array}{l}\begin{array}{l}\text { Friction Factor } \\
\text { Ratios, } / / f_{\text {trap }}\end{array} \\
\text { Reynolds Number }\end{array}$}} \\
\hline & & & & & \\
\hline & & 9,400 & 39,000 & 9,400 & 39,000 \\
\hline \multirow[t]{2}{*}{$\operatorname{Sin} \theta$} & 20 & 4.75 & 3.88 & 0.993 & 1.019 \\
\hline & 10 & & & 0.989 & 1.017 \\
\hline \multirow[t]{2}{*}{ Semicircle } & 20 & 4.69 & 3.25 & 0.968 & 0.959 \\
\hline & 10 & & & 0.962 & 0.964 \\
\hline \multirow[t]{2}{*}{ Arc } & 20 & 4.41 & 2.81 & 0.959 & 0.936 \\
\hline & 10 & & & 0.948 & 0.885 \\
\hline \multirow[t]{2}{*}{ Trapezoid } & 20 & 4.94 & 3.72 & 1.000 & 1.000 \\
\hline & 10 & & & 1.000 & 1.000 \\
\hline
\end{tabular}

\subsubsection{Local Pressure Distrlbutions}

Figure 7.28 presents the local pressure-drop distributions at the wall, the centerline, and the radial average for a sine-shaped rib. The reference pressure is the centerline pressure at the inlet. There is a large radial pressure gradient, even for a fully developed flow field. The condition of a radially uniform pressure distribution does not exist as for the case of a plain or unenhanced tube. The very sharp drops of the wall pressure at the disruption and the recovery that follows should be noted. There is a slight increase in the centerline pressure just in front of the disruption, followed by a decrease. The average pressure drop decreases at the disruption and is followed by some pressure recovery.

The wall pressure drops are shown in Figures 7.29 and 7.30 for Reynolds numbers of 9,400 and 39,000 , respectively. The entire wall pressure field is affected by the disruption shape. The pressure drop at the rib increases for the more contoured shapes. The greater pressure recovery away from the rib when the rib is less contoured is the major reason for the overall pressure drop decrease that occurs with a decrease in the surface contour.

\subsubsection{Heat-Transfer Enhancement Level}

The heat-transfer performance of the sine-shaped disruption for two Reynolds numbers is shown in Figure 7.31. The enhancement levels increase in the vicinity of the disruption but decrease in the boundary-layer development region downstream of the reattachment point. Again, 


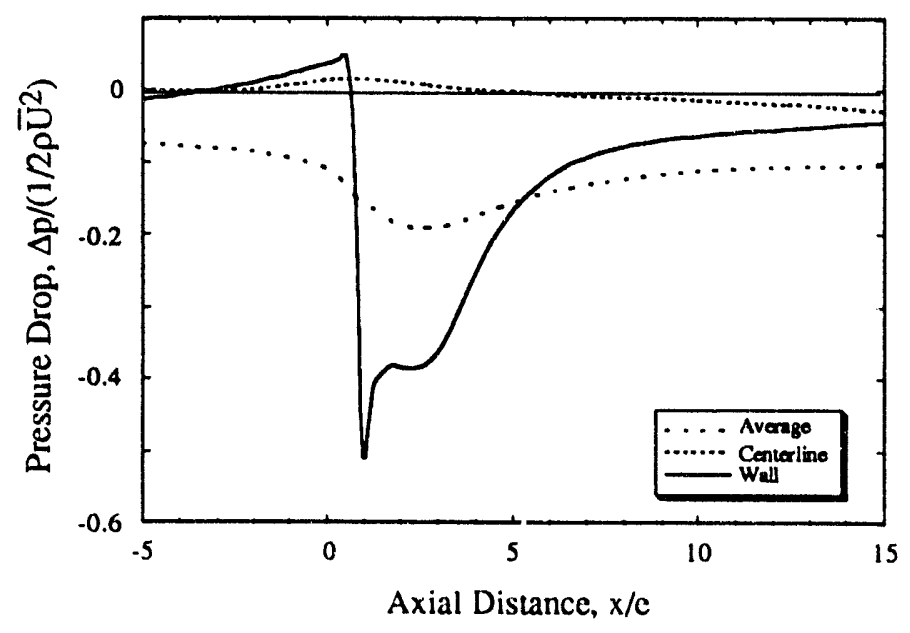

FIGURE 7.28 Pressure-Drop Distributions for a Sine-Shaped Disruption: $\operatorname{Re}=9,370, p / e=20$, and $w / e=2.0$

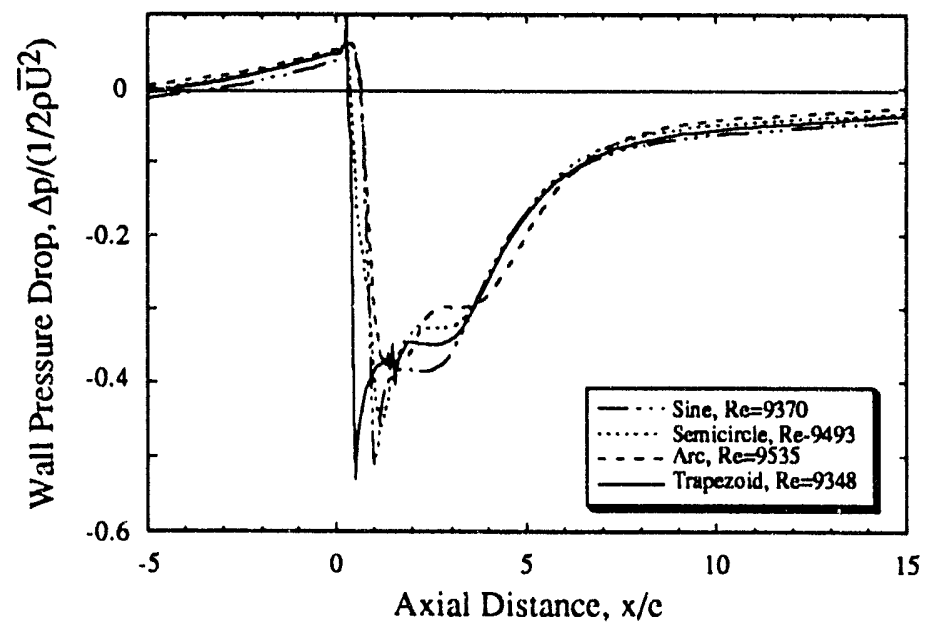

FIGURE 7.29 Comparison of the Pressure

Distributions for the Smaller Reynolds Number:

$p / e=20$ 


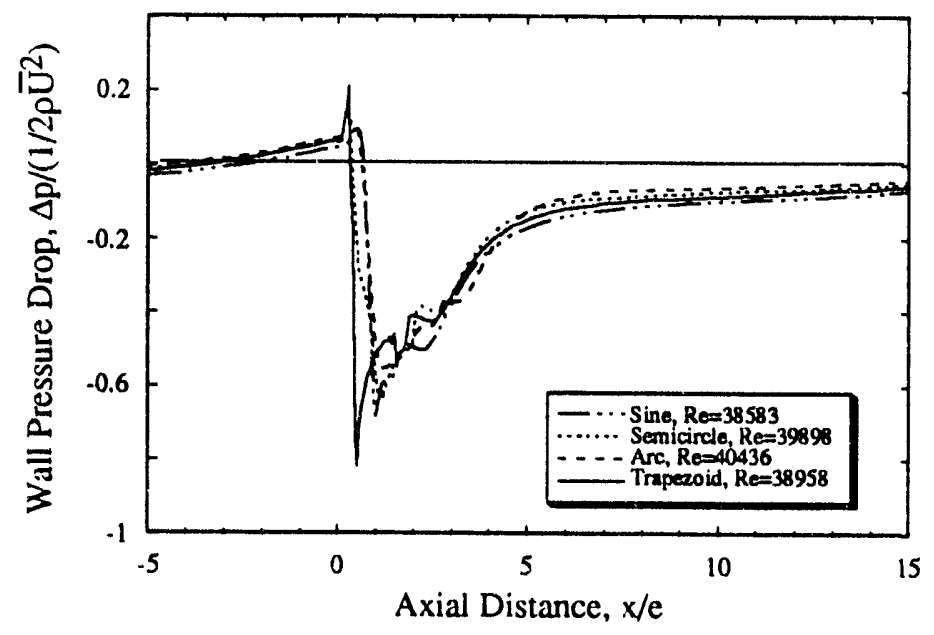

FIGURE 7.30 Comparison of the Pressure Distributions for the Larger Reynolds Number: $p / e=20$

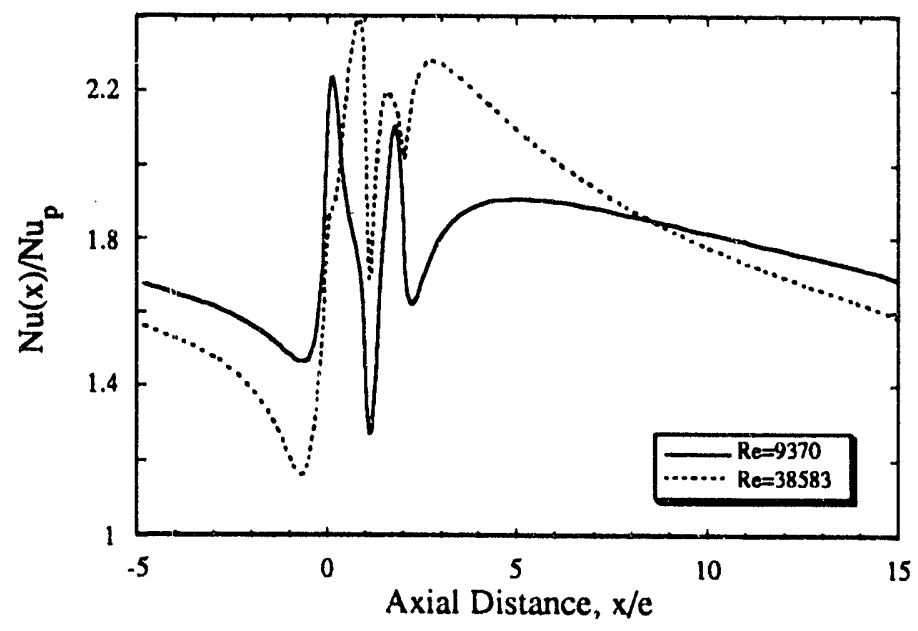

FIGURE 7.31 Heat-Transfer Enhancement Level for a Sine-Shaped Disruption: $p / e=20$ and $w / e=2.0$ 
this pattern was obtained for all shapes. This trade-off is the reason that the enhancement level increases to a maximum value with increasing Reynolds number. The maximum level depends on the disruption height, pitch, shape, and the Prandtl number. It is also apparent that the contribution from the downstream recirculation region increases with increasing Reynolds number, and the magnitude of heat-transfer maximum in this region also increases. The heat-transfer maximum located in the recirculation region moves toward the rib with an increase in Reynolds number. These points of maxima are located well within the recirculation region and are between the reattachment points and the points of the maximum shear stresses.

The effect of the disruption shape on the local Nusselt numbers is presented in Figures 7.32 and 7.33 at Reynolds numbers of about 9,400 and 39,000, respectively. The variations caused by the different shapes are mostly concentrated in the region of the disruption. It is readily apparent, then, that the influence of the disruption shape on the thermal performance should decrease as the pitch increases. The heat-transfer enhancement levels in the recirculation zone, in the boundary layer development zone, and in the front recirculation zone increase when the surface-contour of the rib becomes more contoured. Also, the point of the maximum heat transfer in the downstream recirculation zone moves toward the rib as the rib surface becomes more contoured.

Figures 7.32 and 7.33 show that the local heat-transfer performance in the rib region is very complex. There are three maxima for the semicircle, arc, and trapezoid shapes and two for the sine shape. For the lower Reynolds number, the magnitudes of the middle maxima are the smallest for the semicircle-shaped, arc-shaped, and trapezoid-shaped ribs; however, the magnitudes become the largest of the three maxima at the higher Reynolds number.

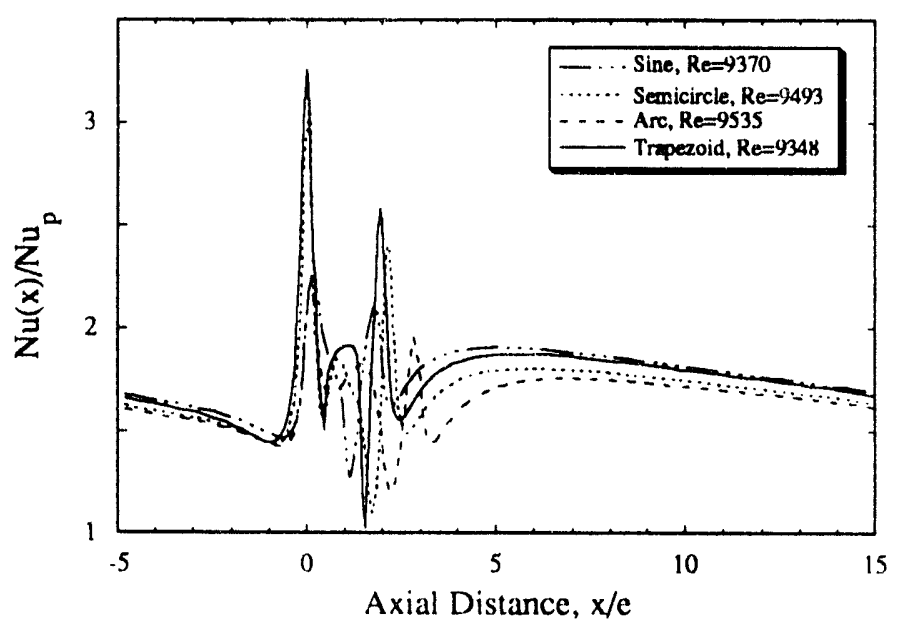

FIGURE 7.32 Comparison of the Heat-Transfer Enhancement Levels for the Smaller Reynolds Number: $p / e=20$ and $e / D=0.002$ 


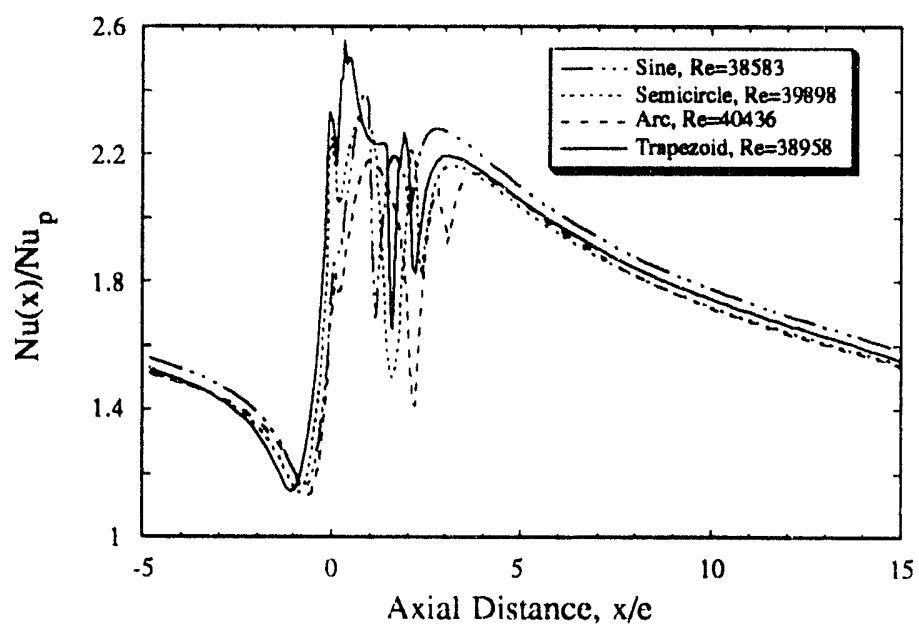

HIGURE 7.33 Comparison of the Heat-Transfer Enhancement Levels for the Larger Reynolds Number: $p / e=20$ and $e / D=0.002$

\subsubsection{Effect of Pitch}

The difference in the overall thermal performance resulting from the different shapes is displayed in Table 7.2. This table shows the mean enhancement level, $\left(\mathrm{Nu} / \mathrm{Nu} \mathrm{p}_{\mathrm{p}}\right)$, and enhancement level ratio with respect to the trapezoid shape as the base for $p / e$ values of 10 and 20 . In general, there is a bigger shape effect - a departure from unity - for the smaller $p / e$ value. This phenomenon is expected because the effect of the shape on the local heat-transfer coefficient is concentrated in the region of the disruption and has a lesser effect in the boundary-layer development region downstream of the reattachment point. 
TABLE 7.2 Comparison of the Mean Nusselt Numbers

\begin{tabular}{|c|c|c|c|c|c|}
\hline \multirow[b]{2}{*}{ Shape } & \multirow[b]{2}{*}{$p / \theta$} & \multicolumn{2}{|c|}{$R_{\theta}=9,400$} & \multicolumn{2}{|c|}{$R_{\theta}=39,000$} \\
\hline & & $\mathrm{Nu} / \mathrm{Nu}_{\mathrm{p}}$ & $\frac{\mathrm{Nu} / \mathrm{Nup}}{(\mathrm{Nu} / \mathrm{Nup})_{\text {trap }}}$ & $\mathrm{Nu} / \mathrm{Nup}$ & $\frac{N u / N u_{p}}{\left(N u / N u_{p}\right)_{\text {trap }}}$ \\
\hline $\operatorname{Sin} \theta$ & $\begin{array}{l}10 \\
20\end{array}$ & $\begin{array}{l}2.1674 \\
1.8419\end{array}$ & $\begin{array}{l}0.945 \\
0.974\end{array}$ & $\begin{array}{l}2.2203 \\
1.8848\end{array}$ & $\begin{array}{l}0.958 \\
0.988\end{array}$ \\
\hline Semicircle & $\begin{array}{l}10 \\
20\end{array}$ & $\begin{array}{l}2.1266 \\
1.7920\end{array}$ & $\begin{array}{l}0.927 \\
0.947\end{array}$ & $\begin{array}{l}2.2143 \\
1.8347\end{array}$ & $\begin{array}{l}0.955 \\
0.961\end{array}$ \\
\hline Arc & $\begin{array}{l}10 \\
20\end{array}$ & $\begin{array}{l}1.9826 \\
1.7118\end{array}$ & $\begin{array}{l}0.864 \\
0.905\end{array}$ & $\begin{array}{l}2.1158 \\
1.7849\end{array}$ & $\begin{array}{l}0.913 \\
0.935\end{array}$ \\
\hline Trapezoid & $\begin{array}{l}10 \\
20\end{array}$ & $\begin{array}{l}2.2941 \\
1.8906\end{array}$ & $\begin{array}{l}1.000 \\
1.000\end{array}$ & $\begin{array}{l}2.3183 \\
1.9083\end{array}$ & $\begin{array}{l}1.000 \\
1.000\end{array}$ \\
\hline
\end{tabular}




\section{Conclusions}

The most important conclusion of this research effort is that numerical analysis can be successfully used to investigate the thermal-hydraulic performance of enhanced passages and, in particular, heat-exchanger tubes. This conclusion applies for both laminar and turbulent singlephase flow, although this report concentrates on the vastly more difficult turbulent case. However, this conclusion is valid for the following case: a two-dimensional, axisymmetric flow field exists with the enhancement mechanism selected for this demonstration - the separation and reattachment mechanism. For the three-dimensional swirl-induced mechanisms, there are as yet no successful demonstrations of the use of numerical analysis to predict the turbulent thermalhydraulic performance of enhanced heat-exchanger tubes.

This research and the study by Chon and Patel (1989) show that the two-layer turbulence model of Chen and Patel (1988) is capable of predicting the general fluid mechanics of the flows with the separation and reattachment mechanism. This model has not, however, been validated with more detailed data for the turbulence stresses and kinetic energy in different flow fields; therefore, further investigation is needed. To the authors' knowledge, this model has not been as extensively tested for heat-transfer calculations. This model predicts the local and mean Nusselt numbers well within the experimental error range, but there is a tendency to underpredict the mean heat-transfer performance as the Reynolds number increases. As discussed in Section 3, the turbulent heat-transfer closure model used is a zero-equation model (i.e., a constant turbulent Prandtl number is assumed). Previous data show that this assumption is marginal even for smooth-tube flows. Therefore, the authors feel that an independent modeling of turbulent heat fluxes will be a useful extension of this study.

The next numerical modeling effort will address enhancement and fouling interactions. This task, which is planned for FY 1992, will be the first attempt to use numerical modeling to analyze the very important influence of particulate fouling on the performance of enhanced passages.

The remainder of this section summarizes the results obtained from the investigation into the influence of the Prandtl number and the disruption shape on the thermal-hydraulic performance of enhanced tubes with transverse disruptions.

\subsection{Influence of the Prandtl Number}

Some fundamental findings about the influence of the Prandtl number on the thermal performance of enhanced tubes with the separation and reattachment mechanism are as follows:

- Six distinct zones must be considered, although some are far more important than others in their contributions to the heat-transfer performance. 
- Two major maximums in the thermal performance exist at the top surface of the rib and in the downstream recirculation zone.

- An increasing Prandtl number leads to a substantial increase of the heat-transfer enhancement at the rib. For high Pr fluids, the mean thermal performance is dominated by what happens at the rib.

- The mean thermal performance exhibits the same variation as the maximum enhancement level at the rib when plotted as a function of the roughness Reynolds number, $\mathrm{e}^{+}$.

- The hea-transfer maximums in the downstream recirculation zone move toward the rib with increasing Reynolds and Prandtl numbers. The location is between the reattachment point and the point of the maximum wall shear stress.

- The influence of the Prandtl number on the thermal performance in the downstream recirculation zone should be and is identical to that at the rib.

- The cause of the heat-transfer improvement in the downstream recirculation zone is the high turbulence levels near the surface.

- Two additional maximums occur at large roughness Reynolds numbers in the front recirculation zone and on the rear face of the rib.

- The location of the reattachment point moves closer to the rib with increasing Reynolds number.

\subsection{Effects of the Disruption Shape}

The important findings obtained by considering the effect of four different shapes - sine, semicircle, arc, and trapezoid - are summarized below:

- Both the pressure drop and heat transfer decrease with an increase in the contour of the disruption.

- The impact of the shape on the thermal-hydraulic performance is concentrated in the region of the disruption, and it has a smaller effect on the performance in the boundary-layer development region. 
- With an increasing pitch, the effect of the shape on heat transfer becomes less important.

- With an increasing disruption contour, the friction factor becomes more Reynolds-number-dependent, reaches the fully rough regime at larger Reynolds numbers, and follows the pattern of decreasing relative roughness displayed in the Moody friction-factor chart.

- The major contribution to the total pressure drop is almost always the form drag, the exceptions being very large pitches where the frictional contribution increases and approaches the plain-tube skin-friction value. 


\section{References}

Amano, R.S., 1983, A Study of Turbulence Downstream of an Abrupt Pipe Expansion, American Institute of Aeronautics and Astronautics Journal, 21:1400-1405.

Amano, R.S., and P. Goel, 1984, A Numerical Study of a Separating and Reattaching Flow by Using Reynolds-Stress Closure, Numerical Heat Transfer, 7:343-357.

Amano, R.S., M.K. Jensen, and P. Goel, 1983, A Numerical Investigation of Turbulent Heat Transport Downstream from an Abrupt Pipe Expansion, Transactions of American Society of Mechanical Engineers, Journal of Heat Transfer, 105:862-869.

Anderson, D.A., J.C. Tannehill, and R.H. Pletcher, 1984, Computational Fluid Mechanics and Heat Transfer, Hemisphere Publishing Co., Washington, D.C.

Aris, R., 1962, Vectors, Tensors, and the Basic Equations of Fluid Mechanics, Prentice-Hall, Englewood Cliffs, N.J.

Arman B., and T.J. Rabas, 1991, Prediction of the Pressure Drop in Transverse, Repeated-Rib Tubes with Numerical Modeling, Fouling and Enhancement Interactions, American Society of Mechanical Engineers, HTD 164:93-99.

Baldwin, B., and H. Lomax, 1978, Thin-Layer Approximation and Algebraic Model for Separated Turbulent Flows, American Institute of Aeronautics and Astroriautics Paper 78-257.

Barba, A., 1984, The Prediction of Convective Heat Transfer in Spirally Fluted Tubes, Ph.D. Thesis, Victoria University of Manchester, U.K.

Baughn, J.W., and X. Yan, 1991, An Insertion Technique Using the Transient Method with Liquid Crystals for Heat Transfer Measurements in Ducts, Fouling and Enhancement Interactions, American Society of Mechanical Engineers, HTD-164:77-84.

Baughn, J.W., and X. Yan, 1992, Local Heat Transfer Measurements in Square Ducts with Transverse and Diagonal Ribs, Enhanced Heat Transfer. American Society of Mechanical Engineers, HTD-202:1-8.

Baughn, J.W., and J.L. Roby, 1992, Enhanced Turbulent Heat Transfer in Circular Ducts with Transverse Ribs, Enhanced Heat Transfer, American Society of Mechanical Engineers, HTD209:9-16. 
Becker, B.R., and R.B. Rivir, 1989, Computation of the Flow Field and Heat Transfer in a Rectangular Passage with a Turbulator. American Society of Mechanical Engineers Paper 89-GT30.

Berger, F.P., and K.-F., F.-L. Hau, 1979, Local Mass/Heat Transfer Distribution on Surfaces Roughened with Small Square Ribs, International Journal of Heat and Mass Transfer, 22:16451656.

Boyd, L.W., et al., 1983, Efficiency Improvement at Gallatin Unit 1 with Corrugated Condenser Tubing, American Society of Mechanical Engineers Paper 83-JPGC-Pwr-4.

Burck, E., 1970. The Influence of Prandtl Number on Heat Transfer and Pressure Drop of Artificially Roughened Channels, Augmentation of Convective Heat and Mass Transfer, American Society of Mechanical Engineers Symposium Volume, pp. 27-35.

Carnavos, T.C., 1979, Cooling Air in Turbulent Flow with Internally Finned Tubes, Heat Transfer Engineering, 1(2):41-46.

Camavos, T.C., 1980, Heat Transfer Performance of Internally Finned Tubes in Turbulent Flow, Heat Transfer Engineering, 1(4):32-37.

Chen, H.C., and V.C. Patel, 1988, Near-Wall Turbulence Models for Complex Flows Including Separation, American Institute of Aeronautics and Astronautics Journal, 26:641-648.

Chien, K.Y., 1982, Predictions of Channel and Boundary-Layer Flows with a Low-Reynolds Number Turbulence Model, American Institute of Aeronautics and Astronautics Journal, 20:33-38.

Chieng, C.C., and B.E. Launder, 1980, On the Calculation of Turbulent Heat Transport Downstream from an Abrupt Pipe Expansion, Numerical Heat Transfer, 3:189-207.

Chon, J.T., and V.C. Patel, 1989, A Numerical Study of Turbulent Flow in a Channel with a Wavy Wall, Proceedings of the 10th Australian Fluid Mechanics Conference, Melbourne, Australia.

Chyu, M.K., and V. Natarajan, 1991, Local Heat/Mass Transfer Distributions on the Surfaces of a Wall-Mounted Cube, Transactions of American Society of Mechanical Engineers, Joumal of Heat Transfer, 1/3:851-857.

Ciofalo, M., and M.W. Collins, 1989, $k-\varepsilon$ Predictions of Heat Transfer in Turbulent Recirculating Flows Using an Improved Wall Treatment, Numerical Heat Transfer, 15:21-47. 
Dawson, D.A., and O. Trass, 1972, Mass Transfer at Rough Surfaces, International Journal of Heat and Mass Transfer, 15:1317-1336.

Dipprey, D.F., and R.H. Sabersky, 1963, Heat and Momentum Transfer in Smooth and Rough Tubes at Various Prandtl Numbers, International Joumal of Heat and Mass Transfer, 6:329-353.

Eimer, K., 1982, Preliminary Experience with Cleaning of Enhanced Heat Exchanger Tubes, Taprogge AG, West Germany, Report C77-3.

Fodemski, T.R., and M.W. Collins, 1988, Flow and Heat Transfer Simulations for Two- and Three-Dimensional Smooth and Ribbed Channels, Proceedings of the Second U.K. National Conference on Heat Transfer, C138/88, University of Strathclyde, Glasgow, U.K., pp. 845-860.

Fujita, H., Y. Hajime, and C. Nagata, 1986, The Numerical Heat Prediction of Fully Developed Turbulent Flow and Heat Transfer in a Square Duct with Two Roughened Facing Walls, Proceedings of the Eighth International Heat Transfer Conference, San Francisco, Calif.

Ganeshan, S., and M. Raja Rao, 1982, Studies on Thermohydraulics of Single and Multi-Start Spirally Corrugated Tubes for Water and Time-Independent Power Law Fluids, International Journal of Heat and Mass Transfer, 25(7):1013-1022.

Gaskell, P.H., and A.K.C. Lau, 1988, Curvature-Compensated Convective Transport: Smart, A New Boundedness-Preserving Transport Algorithm, International Journal for Numerical Methods in Fluids, 8:617-641.

Gooray, A.M., C.B. Watkins, and W. Aung, 1985, Turbulent Heat Transfer Computations for Rearward-Facing Steps and Sudden Pipe Expansions, Transactions of American Society of Mechanical Engineers, Journal of Heat Transfer, 107:70-76.

Gowen, R.A., and J.W. Smith, 1968, Turbulent Heat Transfer from Smooth and Rough Surfaces, International Journal of Heat and Mass Transfer, 11:1657-1673.

Gupta, R.H., and M. Raja Rao, 1979, Heat Transfer and Frictional Characteristics of Spirally Enhanced Tubes for Horizontal Condensers, Advances in Enhanced Heat Transfer, American Society of Mechanical Engineers, San Diego, Calif., pp. 11-21.

Han, J.C., L.R. Glicksman, and W.M. Rohsenow, 1978, An Investigation of Heat Transfer and Friction for Rib-Roughened Surfaces, International Journal of Heat and Mass Transfer, $21: 1143$ 1156. 
Han, J.C., J.S. Park, and M.Y. Ibrahim, 1986, Measurement of Heat Transfer and Pressure Drop in Rectangular Channels with Turbulence Promotors, National Aeronautics and Space Administration Contractor Report 4105.

Harlow, F.H., and J.E. Welch, 1965, Numerical Calculation of Time-Dependent Viscous Incompressible Flow of Fluids, Physics of Fluids, 8:2182-2189.

Hijikata, K., and Y. Mori, 1987, Fundamental Study of Heat Transfer Augmentation of Tube Inside Surface by Cascade Smooth Surface-Turbulence Promotors, Wärme und Stoffübertragung, 21:115-124.

Hijikata, K., H. Ishiguro, and Y. Mori, 1987, Heat Transfer Augmentation in a Pipe Flow with Smooth Cascade Turbulence Promoters and Its Application to Energy Conversion, Heat Transfer in High Technology and Power Engineering, W.J. Yang and Y. Mori, eds., Hemisphere Publishing Corp., Washington, D.C., pp. 368-379.

Hill, K., 1978, Enhanced Heat Transfer Tubes, Fairleigh Dickinson University Summer Course 1978: Theory and Practice of Desalination.

Hinze, J.O., 1959, Turbulence, McGraw-Hill Book Company, Inc., New York.

Hitachi Cable, Ltd., 1987, Hitachi High-Performance Heat Transfer Tubes, Chiyoda Bldg., 2-1-2, Marunouchi, Chiyoda-ku, Tokyo 100, Japan.

Houle, J.F., and W.T. Buhrig, 1973, Performance of Freeport, Texas, VTE/MSF Plant, Fourth International Symposium on Fresh Water from the Sea, $1: 313-318$.

Hung, Y.H., T.M. Liou, and Y.C. Syang, 1987, Heat Transfer Enhancement of Turbulent Flow in Pipes with an Internal Circular Rib, Advances in Enhanced Heat Transfer-1987, American Society of Mechanical Engineers, HTD-68:55-64.

Inoue, S., T. Tsuruta, and S. Funshashi, 1975, Multiple Effect VTE Desalination Plant Experiences in Operation and Performance 500 Ton/Day Plant at Innoshima Shipyard, Heat Transfer Japanese Research, 4(5):1-19.

Issa, R.I., A.D. Gosman, and A.P. Watkins, 1986, The Computation of Compressible Recirculating Flows by a Non-Iterative Implicit Scheme, Journal of Computational Physics, 62:66-82.

Johnson, R.W., and B.E. Launder, 1982, Discussion on the Calculation of Turbulent Heat Transport Downstream from an Abrupt Pipe Expansion, Numerical Heat Transfer, 5:493-496. 
Jones, W.P., and B.E. Launder, 1972, The Prediction of Laminarization with a Two-Equation Model of Turbulence, International Journal of Heat and Mass Transfer, 15:301-314.

Kakaç, S., R.K. Shah, and W. Aung, 1987, Handbook of Single-Phase Convective Heat Transfer, John Wiley and Sons, Inc., New York, p. 4.24.

Kalinin, E.K., et al., 1970, The Experimental Study of the Heat-Transfer Intensification Under Conditions of Forced One- and Two-Phase Flow in Channels, Augmentation of Convective Heat and Mass Transfer, American Society of Mechanical Engineers Symposium Volume, pp. 80-90.

Katsman, D., and Y. Kishinevsky, 1983, Spiral, High-Strength, Corrosion-Resistance Tube Application in PWR Power Plant Condensers, American Society of Mechanical Engineers Paper 83-JPGC-Pwr-17.

Kobayashi, M.H., and J.C.F. Pereira, 1991, Calculation of Incompressible Laminar Flows on a Nonstaggered, Nonorthogonal Grid, Numerical Heat Transfer, 19:243-262.

Kornbau, R.W., C.C. Richards, and R.O. Lewis, 1983, Seawater Biofouling Countermeasures for Spirally Enhanced Condenser Tubes, The Institution of Chemical Engineers, Condensers: Theory and Practice, Symposium Series No. 75, pp. 200-212.

Krall, K.M., and E.M. Sparrow, 1966, Turbulent Heat Transfer in the Separated, Reattached, and Redevelopment Regions of a Circular Tube, Transactions of American Society of Mechanical Engineers, Journal of Heat Transfer, 88:131-139.

Lakshminarayana, B., 1986, Turbulence Modeling for Complex Shear Flows, American Institute of Aeronautics and Astronautics Journal, 24:1900-1917.

Lam, C.K.G., and K.A. Bremhorst, 1981, Modified Form of the $k-\varepsilon$ Model for Predicting Wall Turbulence, Transactions of American Society of Mechanical Engineers, Journal of Fluids Engineering, 103:456-460.

Launder, B.E., 1984, Second Moment Closure: Methodology and Practice, in Turbulent Models and Their Applications, Editions Eyrolls, Paris, 2:1-150.

Launder, B.E., 1988, On the Computation of Convective Heat Transfer in Complex Turbulent Flows, Transactions of American Society of Mechanical Engineers, Journal of Heat Transfer, 110:1112-1128.

Launder, B.E., and W.M. Ying, 1972, Secondary Flows in Ducts of Square Cross-Section, Journal of Fluid Mechanics, 54:289-295. 
Launder, B.E., and B.I. Sharma, 1974, Application of Energy Dissipation Model of Turbulence to the Calculation of Flow Near a Spinning Disk, Letters in Heat and Mass Transfer, 18:307-312.

Leonard, B.P., 1979, A Stable and Accurate Corrective Modeling Procedure Based on QuadraticUpstream Interpolation, Computer Methods in App ' ad Mechanics and Engineering, 19:59-98.

Leonard, B.P., 1988, Simple High Accuracy Resolution Program for Convective Modeling of Discontiluities, International Journal for Numerical Methods in Engineering, 30:141-154.

Leonard, B.P., 1991, The ULTIMATE Conservative Difference Scheme Applied to Unsteady One-Dimensional Advection, Computer Methods in Applied Mechanics and Engineering, $88: 17-74$.

Leonard, B.P., and S. Mokhtari, 1990, Beyond First-Order Upwinding: The ULTRA-SHARP Alternative for Non-Oscillatory Steady-State Simulation of Convection, International Journal for Numerical Methods in Engineering, 30:729-760.

Lewis, M.J., 1975a, An Elementary Analysis for Predicting the Momentum-and Heat-Transfer Characteristics of Hydraulically Rough Surfaces, Transactions of American Society of Mechanical Engineers, Journal of Heat Transfer, 97:249-254.

Lewis, M.J., 1975b, Optimizing the Thermohydraulic Performance of Rough Surfaces, International Journal of Heat and Mass Transfer, 18:1243-1248.

Li, H.M., et al., 1982, Investigation of Tube-Side Flow Visualization, Friction Factors and Heat Transfer Characteristics of Helical-Ridging Tubes, Proceedings of the Seventh International Heat Transfer Conference, Hemisphere Publishing Corp., Washington, D.C., 3:75-80.

Lumley, J.L., 1983, Turbulence Modeling, American Society of Mechanical Engineers Journal of Applied Mechanics, 50:1097-1103.

Mah, C.S., 1987, Inwovative Counterflow Gas/Fine Solids Direct Contact Heat Exchanger, Final Report, Aerojet Energy Conversion Co., Sacramento, Calif,, Report AECC 86-JAA-001-F.

Majumdar, M., 1988, Role of Underrelaxation in Momentum Interpolation for Calculation of Flow with Nonstaggered Grids, Numerical Heat Transfer, 13:125-132.

Malhotra, A., and S.S. Kang, 1984, Turbulent Prandtl Number in Circular Pipes, International Journal of Heat and Mass Transfer, 27:2158-2161. 
Mehta, M.H., and M. Raja Rao, 1979, Heat Transfer and Frictional Characteristics of Spirally Enhanced Tubes for Horizontal Condensers, Advances in Enhanced Heat Transfer, San Diego, Calif., pp. 11-22.

Mehta, M.H., and M. Raja Rao, 1988, Analysis and Correlation of Turbulent Flow Heat Transfer and Friction Coefficients in Spirally Corrugated Tubes for Steam Condenser Application, Proceedings of 1988 National Heat Transfer Conference, American Society of Mechanical Engineers, HTD-96(3):307-312.

Mendes, P.R.S., and M.H.P. Mauricio, 1987, Heat Transfer, Pressure Drop, and Enhancement Characteristics of the Turbulent Through Internally Ribbed Tubes, Convective Transport, American Society of Mechanical Engineers, HTD-82:15-22.

Miller, T.F., and F.W. Schmidt,1988, Use of Pressure-Weighted Interpolation Method for the Solution of the Incompressible Navier-Stokes Equations on a Nonstaggered Grid System, Numerical Heat Transfer, 14:213-233.

Mussalli, Y.G., and L.S. Gordon, 1984, Use of Enhanced Heat Transfer Tubes in Power Plant Condensers, Heat Transfer in Heat Rejection Systems, American Society of Mechanical Engineers, HTD-37:27.

Myong H.K., and N. Kasagi, 1990, A New Approach to the Improvement of $k-\varepsilon$ Turbulence Model for Wall-Bounded Shear Flows, Japanese Society of Mechanical Engineers International Journal II, 33:63-72.

Nagano Y., and M. Tagawa, 1990, An Improved k-E Model for Boundary Layer Flows, Transactions of American Society of Mechanical Engineers, Journal of Fluids Engineering, 112:33-39.

Nakayama, W., K. Takahashi, and T. Daikoku, 1983, Spiral Ribbing to Enhance Single-Phase Heat Transfer Inside Tubes, American Society of Mechanical Engineers-Japanese Society of Mechanical Engineers Thermal Engineering Joint Conference Proceedings, Honolulu, Hawaii, pp. 365-372.

Nallasamy, M., 1987, Turbulence Models and Their Applications to the Prediction of Internal Flows: A Review, Computers and Fluids, 15:151-194.

Newson, I.H., 1976, Enhanced Heat Transfer Condenser Tubing for Advanced Multistage Flash Distillation Plants, Fifth International Symposium on Fresh Water from the Sea, 2:107-115.

Nikolaev, G.V., et al., 1982, Comparative Studies of a 200 KTSS-2 Condenser with Smooth and. with Rolled Tubes, Energomashinostroenie, No. 1, pp. 15-16. 
Nunner, W., 1956, Heat Transfer and Pressure Drop in Rough Tubes, United Kingdom Atomic Energy Authority, Harwell, Atomic Energy Research Energy Establishment Lib/Trans. 786 (1958).

Ota, T., and H. Nishiyama, 1987, A Correlation of Maximum Turbulent Heat-transfer coefficient on Reattachment Flow Region, International Journal of Heat and Mass Transfer, 30(6):1193-1200.

Panchal, C.B., 1989, Experimental Investigation of Seawater Biofouling for Enhanced Surfaces, Heat Transfer Equipment Fundamentals, Design, Applications and Operating Problems, American Society of Mechanical Engineers, HTD-108:231-238.

Panchal, C.B., and D.M. France, 1992, unpublished information.

Patankar, S.V., 1980, Numerical Heat Transfer and Fluid Flows, McGraw.Hill, New York.

Patankar S., and D.B. Spalding, 1972, A Calculation Procedure for Heat, Mass and Momentum Transfer in Three-Dimensional Parabolic Flows, International Journal of Heat and Mass Transfer, 15:1887-1906.

Patankar, S.V., C.H. Liu, and E.M. Sparrow, 1977, Fully Developed Flow and Heat Transfer in Ducts Having Streamwise-Periodic Variations of Cross-Sectional Area, Transactions of American Society of Mechanical Engineers, Joumal of Heat Transfer, 99:180-186.

Patankar, S.V., M. Invanovic, and E.M. Sparrow, 1979, Analysis of Turbulent Flow and Heat Transfer in Internally Finned Tubes and Annuli, Transactions of American Society of Mechanical Engineers, Journal of Heat Transfer, 101:29-37.

Patel, V.C., 1992, University of Iowa, private communication.

Patel, V.C. , 1973, A United View of the Law of the Wall Using Mixing Length Theory, The Aeronautical Quarterly, 24:55-70.

Patel, V.C., W. Rodi, and G. Scheverer, 1985, Turbulence Models for Near-Wall and Low Reynolds Number Flows: A Review, American Institute of Aeronautics and Astronautics Journal, 23:1308-1319.

Peric, M., R. Kessler, and G. Scheuerer, 1988, Comparison of Finite Volume Numerical Methods with Staggered and Colocated Grids, Computers and Fluids, 16:389-403.

Petukov, B.S., 1970, Heat Transfer and Friction Factor in Turbulent Pipe Flow with Variable Physical Properties, Advances in Heat Transfer, 6:503-564. 
Phillips, R.E., and F.W. Schmidt, 1985a, Multigrid Techniques for the Solution of Passive Scalar Advection-Diffusion Equation, Numerical Heat Transfer, 8:25-43.

Phillips, R.E., and F.W. Schmidt, 1985b, A Multilevel-Multigrid Technique for Recirculating Flows, Numerical Heat Transfer, 8:573-594.

Prandtl, L., 1925, Uber die Ausgebildete Turbulenz, Zeitschrift für angenwandte Mathematik und Mechanik, 5:136-139.

Rabas, T.J., 1989, Selection of the Energy-Efficient Enhancement Geometry for Single-Phase Turbulent Flow Inside Tubes, Heat Transfer Equipment Fundamentals, Design, Applications and Operating Problems, National Heat Transfer Conference, American Society of Mechanical Engineers, HTD-108:193-204.

Rabas, T.J., A.E. Bergles, and D.L. Moen, 1988, Heat Transfer and Pressure Drop Correlations for Spirally Grooved (Rope) Tubes Used in Surface Condensers and Multistage Flash Evaporators, 1988 National Heat Transfer Conference, American Society of Mechanical Engineers, HTD-96:693-704.

Rabas, T.J., et al., 1990, Heat-Rate Improvements Obtained with the Use of Enhanced Tubes in Surface Condensers, Electric Power Research Institute Condenser Technology Conference, Boston, Mass.

Rabas, T.J., et al., 1991, Comparison of Power-Plant Condenser Cooling-Water Fouling Rates for Spirally Indented and Plain Tubes, Fouling and Enhancement Interactions, American Society of Mechanical Engineers, HTD-164:29-37.

Ravigururajan, T.S., and A.E. Bergles, 1985, General Correlations for Pressure Drop and Heat Transfer for Single-Phase Turbulent Flow in Internally Ribbed Tubes, Augmentation of Heat Transfer in Energy Systems, American Society of Mechanical Engineers, HTD-52:9-20.

Ravigururajan, T.S., and A.E. Bergles, 1986a, An Experimental Verification of General Correlations for Single-Phase Turbulent Flow in Ribbed Tubes, Advances in Heat Exchanger Design, American Society of Mechanical Engineers, HTD-66:1-11.

Ravigururajan, T.S., and A.E. Bergles, 1986b, Study of Water-Side Enhancement for Ocean Thermal Energy Conversion Heat Exchangers, Final Report, College of Engineering, Iowa State University.

Rebello, W.J., 1987, Assessment of Heat Transfer Enhancement and Fouling in Industrial Heat Exchangers, U.S. Department of Energy Report DOE/CE/40716-3 (DE89005620). 
Renfftlen, R.G., 1991, On-Line Sponge Ball Cleaning of Enhanced Heat Transfer Tubes, Fouling and Enhancement Interactions, American Society of Mechanical Engineers, HTD-164:55-60.

Reynolds, A.J., 1975, The Prediction of Turbulent Prandtl and Schmidt Numbers, International Journal of Heat and Mass Transfer, 18:1055-1068.

Reynolds, W.C., 1976, Computation of Turbulent Flows, Annual Review of Fluid Mechanics, 8:183-208.

Reynolds, W.C., 1984, Physical and Analytical Foundations, Concepts and New Directions in Turbulence Modeling and Simulation, in Turbulence Models and Their Applications, 2:151-294, Editions Eyrolls, Paris.

Rhie, C.M., 1981, A Numerical Study of The Flow Past an Isolated Airfoil with Separation, Ph.D. dissertation, University of Illinois, Urbana-Champaign.

Rhie, C.M., and W.L. Chow, 1983, Numerical Study of the Turbulent Flow Past an Airfoil with Trailing Edge Separation, American Institute of Aeronautics and Astronautics Journal, 21:15251532.

Rodi, W., 1980, Turbulence Models and Their Applications in Hydraulics, State-of-the-Art Paper, International Association for Hydraulics Research, Delft, The Netherlands.

Rodi, W., 1987, Examples of Calculation Methods for Flow and Mixing in Stratified Fluids, Geophysical Research, 92:5305-5328.

Rodi, W., 1991, Experience with Two-Layer Models Combining the k-E Model with a OneEquation Model Near the Wall, American Institute of Aeronautics and Astronautics Paper 91-0216.

Rowe, M., 1983, Power Plant Condensers - Recent CEGB Experience, The Institution of Chemical Engineers, Condensers: Theory and Practice, Symposium Series No. 75, pp. 113-134.

Savage, D.W., and J.E. Myers, 1963, The Effect of Artificial Surface Roughness on Heat and Momentum Transfer, American Institute of Chemical Engineers Journal, 9:694-702.

Scherer, V., and S. Wittig, 1989, The Influence of the Recirculation Region: A Comparison of the Convective Heat Transfer Downstream of a Backward-Facing Step and Behind a Jet in Cross Flow, American Society of Mechanical Engineers Paper 89-GT-59. 
Sethumadhavan, R., and M. Raja Rao, 1983, Turbulent Flow Heat Transfer and Fluid Friction in Helical-Wire-Coil-Inserted Tubes, International Journal of Heat and Mass Transfer, 26:18331845.

Smith, J.W., and R.A. Gowen, 1965, Heat Transfer Efficiency in Rough Pipes at High Prandtl Number, American Institute of Chemical Engineers Journal, 11:941-943.

Spalding, D.B., 1972, A Novel Finite-Difference Formulation for Differential Equations Involving both First and Second Derivatives, International Journal for Numerical Methods in Engineering, 4:557-559.

Sparrow, E.M., and A.T. Prata, 1983, Numerical Solutions for Laminar Flow and Heat Transfer in a Periodically Converging-Diverging Tube with Experimental Confirmation, Numerical Heat Transfer, 6:441-461.

Sydberger, T., and U. Lotz, 1982, Relation between Mass Transfer and Corrosion in a Turbulent Pipe Flow, Journal of the Electrochemical Society, 129(2):276-283.

Tan, Y,K., and J.W. Xiao, 1989, Influence of Prandtl Number to Fluid Heat-Transfer Characteristics of Spirally Corrugated Tubes, The Fourth Asian Congress of Fluid Mechanics, Hong Kong, August 19-23.

Taylor, C., et al., 1991, Numerical Simulation of Three-Dimensional Turbulent Flow and Heat Transfer within a Multi-Ribbed Cylindrical Duct, American Society of Mechanical Engineers Paper 91-GT-8.

Techo, R., R.R. Tickner, and R.E. James, 1965, An Accurate Equation for the Computation of the Friction Factor for Smooth Pipes from the Reynolds Number, Journal of Applied Mechanics, $32: 443$.

Thompson, J.F. ed., 1982, Numerical Grid Generation, North Holland, Amsterdam.

Thompson, J.F., Z.U.A. Warsi, and C.W. Mastin, 1985, Numerical Grid Generation: Foundations and Applications, North Holland, Amsterdam.

Van Doormal, J.P., and G.D. Raithby, 1984, Enhancements of the SIMPLE Method for Predicting Incompressible Fluid Flows, Numerical Heat Transfer, 7:147-163.

Webb, R.L., 1979, Toward a Common Understanding of the Performance and Selection of Roughness for Forced Convection, in Studies in Heat Transfer: A Festschrift for E.R.G.Eckert, J.P. Hartnett, ed., Hemisphere Publishing Co., Washington, D.C., pp. 252-272. 
Webb, R.L., 1982, Performance, Cost-Effectiveness and Water-Side Fouling Considerations of Enhanced Tube Heat Exchangers for Boiling Service with Tube-Side Water Flow, Heat Transfer Engineering, 3(3-4):84-98.

Webb, R.L. and L.M. Chamra, 1991, On-Line Cleaning of Particulate Fouling in Enhanced Tubes, Fouling and Enhancement Interactions, American Society of Mechanical Engineers, HTD164:47-56.

Webb, R.L., E.R.G. Eckert, and R.L. Goldstein, 1971, Heat Transfer and Friction in Tubes with Repeated-Rib Roughness, International Journal of Heat and Mass Transfer, 14:601-617.

Webb, R.L., L.L. Haman, and T.S. Hui, 1984, Enhanced Tubes in the Electric Utility Steam Condensers, Heat Transfer in Heat Rejection Systems, American Society of Mechanical Engineers, HTD-37:17-26.

Wieland-Werke AG, 1989, private communication from K. Menze to T. Rabas describing enhanced tubes manufactured by Wieland-Werke AG.

Wilkes, N.S., 1981, The Prediction of Heat Transfer from Surfaces Roughened by Transverse Ribs, United Kingdom Atomic Energy Authority, Harwell, Atomic Energy Research Energy Establishment, 10293.

Withers, J.G., 1980, Tube-Side Heat Transfer and Pressure Drop for Tubes Having Helical Internal Ridging with Turbulent/Transitional Flow of Single-Phase Fluid, Part 1, Single-Helix Ridging, Heat Transfer Engineering, 2(1):48-58.

Withers, J.G., and E.H. Young, 1971a, Steam Condensing on Vertical Rows of Horizontal Corrugated and Plain Tubes, Industrial and Engineering Chemistry Process Design and Development, 10:19-30.

Withers, J.G., and E.H. Young, 1971b, New Wrinkle in Condenser Tubing, Power Engineering.

Wolverine Engineering Data Book II, 1984, Wolverine Korodense Tube: General Information and Heat Transfer Design Notes, Bulletin No. 4020.

Yamada, H., and N. Nishijima, 1980, Some Characteristics of Corrugated Titanium Tube, Proceedings 4th International Conference on Titanium, Kyoto, Japan.

Yap, D.R., 1987, Turbulent Heat and Momentum Transfer in Recirculating and Impinging Flows, Ph.D. dissertation, University of Manchester, UK. 
Yorkshire Alloys, Ltd., 1982, YIM Heat Exchanger Tubes: Design Data for Horizontal Rope Tubes in Steam Condensers, Technical Memorandum 3, Yorkshire Imperial Metals, Ltd., Leeds, England.

Young, E.H., G.T.S. Chen, and R. Aggarwal, 1969, The Condensation of Steam on Horizontal Single-Start and Triple-Start Corrugated Tubes, The University of Michigan, Office of Research Administration, Report No. 61.

Young, E.H., W.G. Lampert, and R.H. Griffiths, 1975, The Condensing of Steam on Horizontal Single-Helix Corrugated Tubes, The University of Michigan, Office of Research Administration, Report No. 63.

Young, E.H., et al., 1968, The Condensation of Steam on Horizontal and Corrugated and Bare Tubes, The University of Michigan, Office of Research Administration, Report No. 60.

Zhu, T., 1991, A Low-Diffuse and Oscillation-Free Convection Scheme, Communications in Applied Numerical Methods, 7:225-232. 


\section{Appendix A:}

\section{Burck Correlation}

This appendix explains the method used to make the comparison of the efficiency-index values predicted by the correlation of Burck (1970) with the experimental data of Webb et al. (1971), as shown in Table 2.1. The correlation proposed by Burck is as follows:

$$
\left.\eta=1.25-0.00032 \log \left(\mathrm{e}_{\mathrm{sg}}^{+}\right)+\log \left[(\operatorname{Pr})^{0.33 /\left(\mathrm{e}_{\mathrm{sg}}^{+}\right.}\right)^{0.243}\right]
$$

To calculate the efficiency index, the sand-grain roughness Reynolds number, $\mathrm{e}_{\mathrm{sg}}^{+}$, is required. This quantity is obtained with the following calculation procedure. The first step is to calculate the sand-grain roughness by using the fully rough friction-factor equation:

$$
\mathrm{f}=1 /\left[2 \log \left(\mathrm{D} / \mathrm{e}_{\mathrm{sg}}\right)+1.74\right]^{2}
$$

The measured friction-factor values for all of the tube geometries tested by Webb et al. (1971) were used to calculate the $\mathrm{D} / \mathrm{e}_{\mathrm{sg}}$ with the above equation by using a trial-and-error procedure. Next, the sand-grain roughness Reynolds number was calculated, or $\mathrm{e}_{\mathrm{sg}}^{+}=\left(\mathrm{e}_{\mathrm{sg}} / \mathrm{D}\right) \operatorname{Re}$ $\sqrt{\mathrm{f} / 2}$.

Table 2.1 (see text) compares the efficiency-index values obtained with the above method, $\eta_{\text {Burck}}$, with the values obtained from the experimental data of Webb et al. (1971), $\eta_{\text {Exp }}$. Note that substantial errors exist - for some cases by almosi a factor of two. 


\section{Appendix B:}

\section{Simulation of Turbulence}

The current numerical simulations of turbulence employ either Reynolds-averaged NavierStokes equations or Navier-Stokes equations directly. The Reynolds-averaging procedure requires turbulence (closure) models to close the equations.

\section{B.1 Closure Models}

The current closure models can be classified as (1) zero-equation (algebraic-viscosity) models, (2) one-equation models, (3) two-equation models, and (4) stress-equation models (Reynolds-stress model and algebraic-stress model).

These models do not form an exact theory of turbulence but are based on model assumptions introduced with the aid of empirical information. The first three models use a Boussinesq eddy-viscosity concept; that is, the turbulent stresses are proportional to the mean velocity in analogy to the viscous stresses in laminar flow. All of these models, which have been reviewed extensively by Reynolds (1976), Rodi (1980, 1987), Lumley (1983), Launder (1984), Lakshminarayana (1986), and Nallasamy (1987), are briefly discussed in the following paragraphs.

\section{B.1.1 Algebraic-VIscosity (Mixing-Length) Models}

The algebraic-viscosity models are based on the eddy-viscosity concept or on the assumption of the similarity of the turbulent flow to the laminar flow. The first turbulence model proposed by Prandtl (1925) is still among the most widely used engineering models. The major assumption is flow isotropy. An evaluation of the length scale for different flows is necessary. As a consequence of their simplicity, these models are strictly valid for two-dimensional simple flows; nevertheless, various attempts have been made to use these models in complex flows.

\section{B.1.2 One-Equation Models}

The one-equation models require the solution of an equation for the turbulent kinetic energy. Then, the eddy viscosity is modeled by:

$$
v_{t}=C_{\mu} l_{\mu} \sqrt{k}
$$


where the length scale, $l_{\mu}$, is specified algebraically and is flow-dependent. It is, therefore, difficult to incorporate the length scale empirically for complex flows. These models are not popular because the results are only marginally better than zero-equation models. However, a successful current trend is to use these models with either two-equation or algebraic-stress models (Chen and Patel 1988; Launder 1988; Rodi 1991).

\section{B.1.3 Two-Equation Models}

These models, which are based on the assumption that the turbulence is characterized by one time scale and one length scale, are some of the most widely used in engineeriag calculations. Two equations are necessary to predict these scales. Because of the assumptions of single scales and isotropy, these models fail to capture many features associated with the complex flows, even though they are satisfactory for simple flows. Therefore, empirical modifications for complex flows are necessary. One of the most famous two-equation models is the $k-\varepsilon$ model developed by Jones and Launder (1972), which has been applied to various flows with some success.

\section{B.1.4 Reynolds-Stress Model}

The main practical limitation of two-equation models is the isotropic eddy-viscosity assumption. In actual flows, the velocity scales develop quite differently. To account for the development of the scales, transport equations for the individual stresses must be introduced. The advantage of the Reynolds-stress model is that the terms accounting for curvature, buoyancy, and rotation are directly introduced. Many problems, however, arise in the model equations and during their solutions.

\section{B.1.5 Algebralc-Stress Model}

The solution is not easily obtained with the Reynolds-stress model. Consequently, convection and diffusion terms in Reynolds-stress equations can be simplified to yield algebraic relations for the respective stresses. The resulting model equations still include the effects of curvature, buoyancy, and rotation. 


\section{B.2 Direct Simulation of Turbulence}

\section{B.2.1 Direct Simulation}

A full time-dependent solution of the Navier-Stokes equations can obtain all of the significant scales of the turbulent motion, including the small-scale dissipation features. These simulations are limited to relatively low Reynolds number flows with simple boundary conditions and known initial statistics (Reynolds 1984).

\section{B.2.2 Large-Eddy Simulations}

In recognition of the resolution problems, only the time-dependent, large-scale motions are computed, and the influence of dissipative scales on the large scales is incorporated through a model. The small-scale motion can be modeled by using the local isotropy assumption (Reynolds 1984). 


\section{DISTRIBUTION FOR ANL/ESD-16}

\section{Internal}

ANL Contract Copy

K.S. Macal (7)

ANL Patent Department

B. Arman (50)

ANL Technical Publications Services (3)

\section{External}

U.S. Department of Energy Office of Scientific and Technical Information (12) Manager, U.S. Department of Energy Chicago Operations Office ANL Libraries 

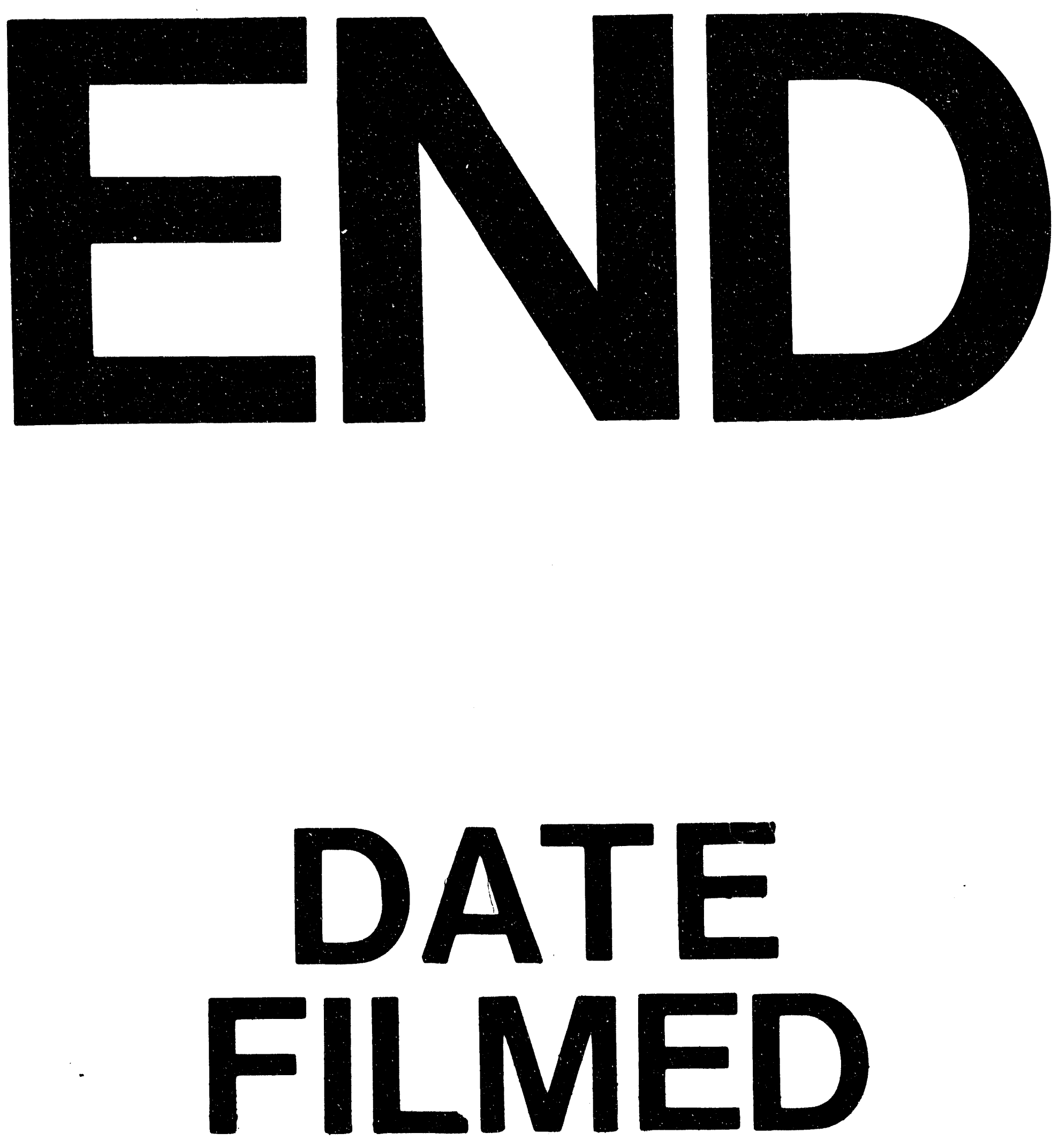

1

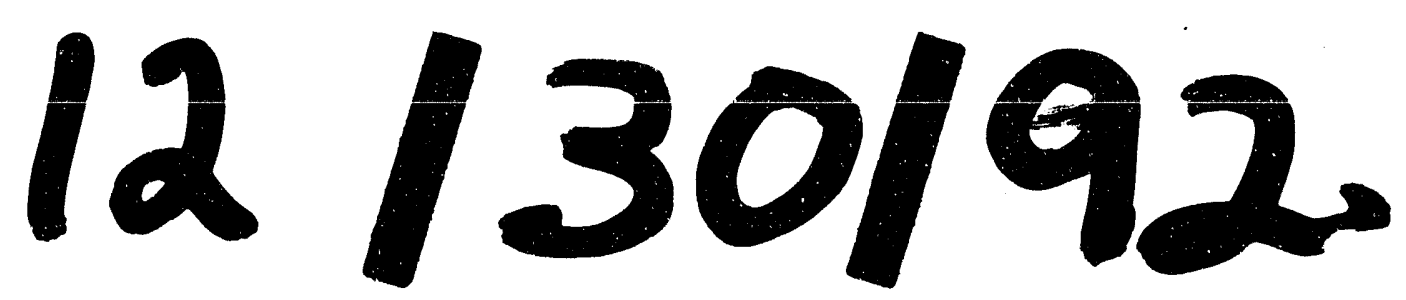


